\title{
Forschungs- und Technologiebericht 2001
}

Lagebericht gem. § 8 FOG über die aus Bundesmitteln geförderte Forschung, Technologie und Innovation in Österreich 
Medieninhaber, Herausgeber, Verleger:

Bundesministerium für Bildung, Wissenschaft und Kultur, 1010 Wien gemeinsam mit:

Bundesministerium für Verkehr, Innovation und Technologie, 1030 Wien

Druck: Ungar Druckerei, 1140 Wien

(C) Wien, 2001 


\section{Vorwort}

Mit dem Bericht über die "Lage von Forschung, Technologie und Innovation in Österreich" wird dem Gesetzgeber eine fundierte Darstellung des österreichischen Innovationssystems vorgelegt, die als Grundlage für die weiteren forschungs- und technologiepolitischen Beratungen und die gesetzliche Beschlussfassung über die weitere Budgetgestaltung dient.

Ausgehend von einer Darstellung der österreichischen Forschungskapazitäten anhand nationaler und internationaler Indikatoren im öffentlichen Bereich (vor allem an den Universitäten) ebenso wie im Unternehmenssektor werden die wichtigsten Felder der Forschungs- und Technologiepolitik, insbesondere die weitere Internationalisierung der österreichischen Forschung und die effektiven Instrumente der Forschungs- und Technologieförderung zur Erreichung des Ziels eines Anteils von 2,5\% der Forschungsausgaben am Bruttoinlandsprodukt analysiert. Speziell wird auch auf die Bedeutung der Informations- und Kommunikationstechnologie eingegangen.

Für die parlamentarische Diskussion ebenso wie für die interessierte Öffentlichkeit bietet der Bericht eine Fülle an Informationen. Durch die gemeinsame Vorlage an den Nationalrat durch die beiden verantwortlichen Ressortministerinnen kommt die wechselseitige Verflechtung der wissenschaftlichen Grundlagenforschung mit der anwendungsorientierten Technologieforschung im Sinne der Verantwortung für ein Nationales Innovationssystem zum Ausdruck.

Elisabeth GEHRER Bundesministerin für Bildung, Wissenschaft und Kultur Dipl.-Ing. Dr. Monika FORSTINGER Bundesministerin für Verkehr, Innovation und Technologie 


\section{Inhaltsverzeichnis}

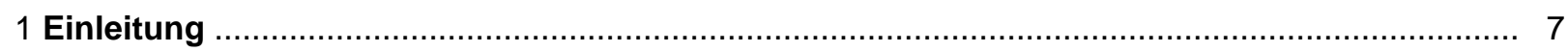

2 Forschungskapazitäten in Österreich: Finanzierung von Forschung und experimenteller Entwicklung 2001 und Forschungspersonal 1998 .................................................................. 9

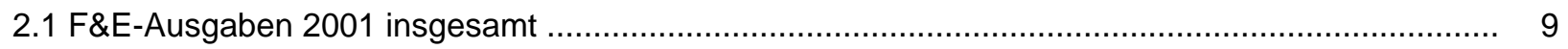

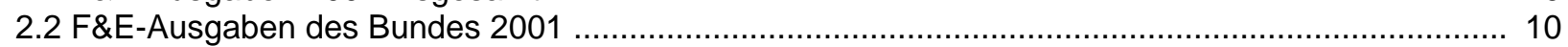

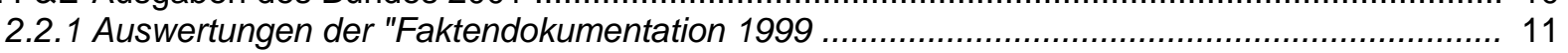

2.3 F\&E-Ausgaben der Bundesländer 2001......................................................................... 13

2.4 Forschung und Entwicklung im Unternehmenssektor ........................................................... 13

2.4.1 Forschungs- und Entwicklungsaktivitäten des Dienstleistungssektors ................................. 16

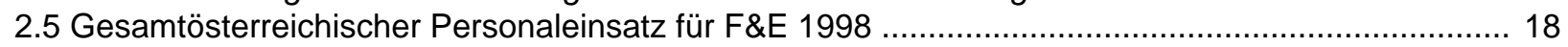

2.5.1 Beschäftigte in F\&E nach Wissenschaftszweigen 1989, 1993 und 1998 .............................. 18

2.5.2 Beschäftigte in F\&E nach Wirtschaftszweigen 1998 ....................................................... 19

2.5.3 Personelle Kapazitäten der universitären Forschung 1998 ............................................... 19

2.5.4 Zusammenfassung des Personaleinsatzes 1998 für F\&E nach Durchführungssektoren ......... 20

3 Forschung und Entwicklung im internationalen Vergleich...................................................... 23

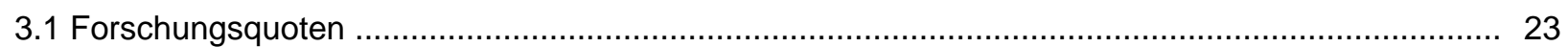

3.2 Finanzierung von Forschung und Entwicklung im internationalen Vergleich ................................ 24

3.3 Durchführung von Forschung und Entwicklung im internationalen Vergleich ................................ 24

3.4 Forschung und Entwicklung im Unternehmenssektor im internationalen Vergleich ....................... 26

3.5 Grundlagenforschung im internationalen Vergleich ................................................................ 27

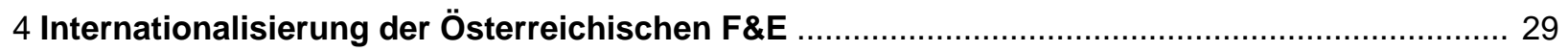

4.1 Die Österreichische Beteiligung am Eu-Rahmenprogramm .................................................... 29

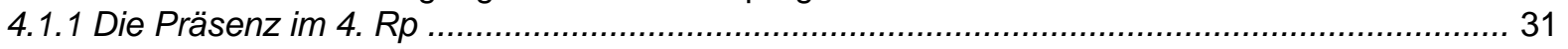

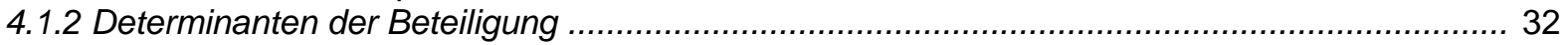

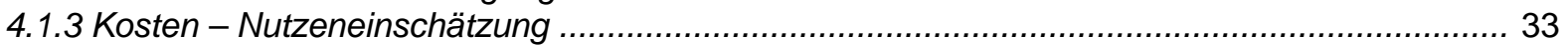

4.1.4 Kurz- und Mittelfristige Wirkungen der Teilnahme ......................................................... 34

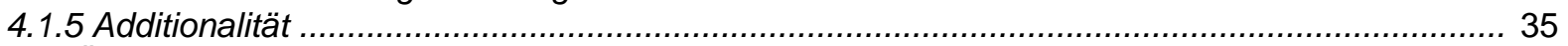

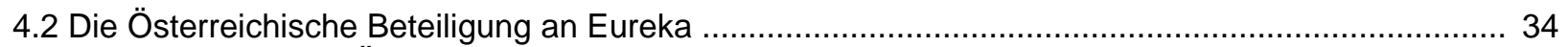

4.2.1 Eckdaten der Österreichischen Beteiligung ................................................................... 36

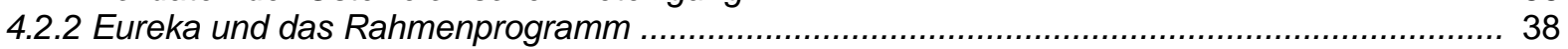

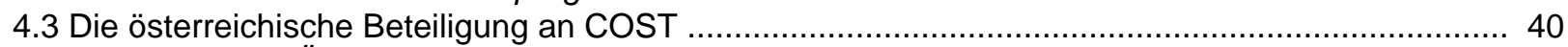

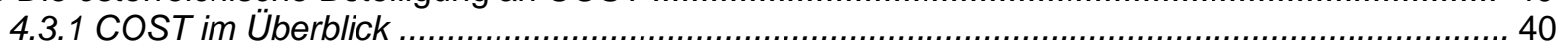

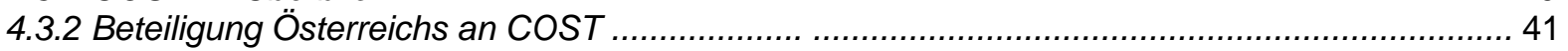

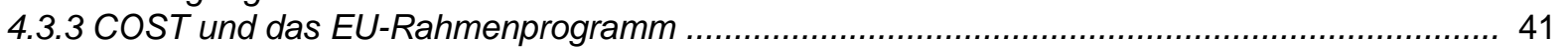

4.4. Die Internationalisierung der Forschung und Entwicklung der Unternehmen ............................... 43

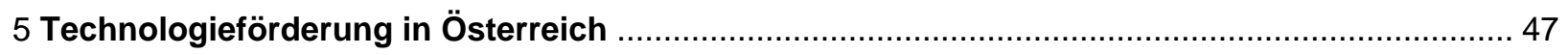

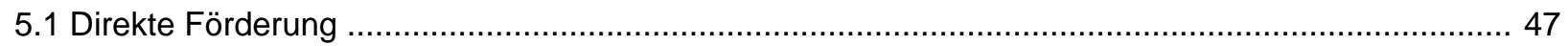

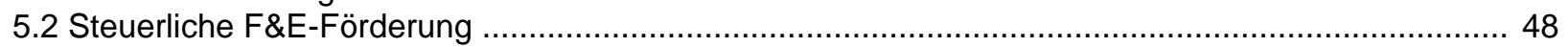

5.3 Quantifizierung der Aggregierten Hebelwirkungen öffentlicher Förderungen ................................ 49

5.3.1 Ökonometrische Abschätzung der Wirkung öffentlicher F\&E-Förderung ................................ 50 
6.1Struktur und Leistungsfähigkeit nach Branchentypen ........................................................... 53

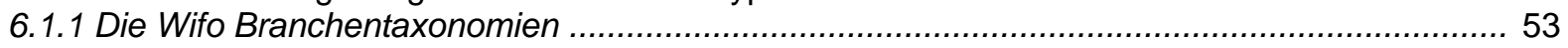

6.1.2 Strukturmerkmale: Produktivität, Wachstum und Qualitätswettbewerb ................................. 54

6.1.3 Eine Österreichische Besonderheit: Gute Performance in Traditionellen Strukturen ................ 55

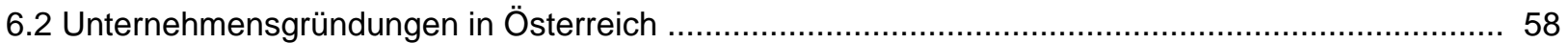

6.3 Das technologische Spezialisierungsprofil: Patentanmeldungen ............................................. 60

7 Informations- und Kommunikationstechnologien (IKT): Die technologische Basis der

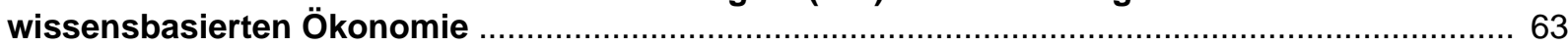

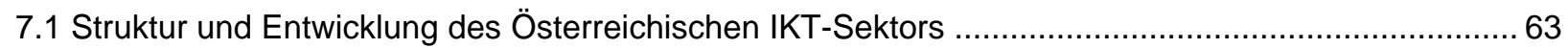

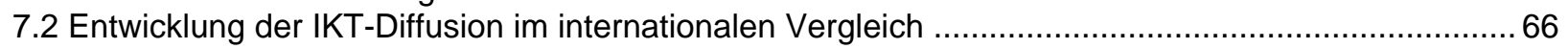

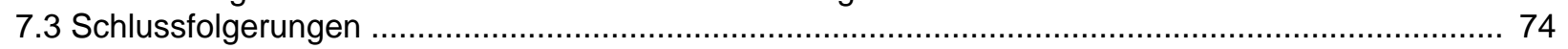

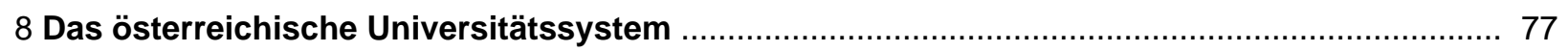

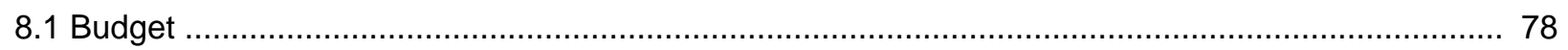

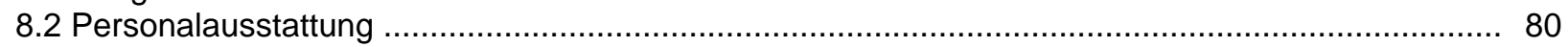

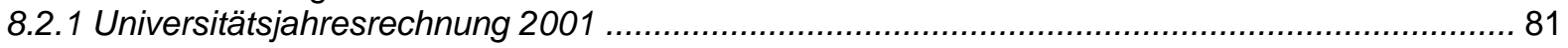

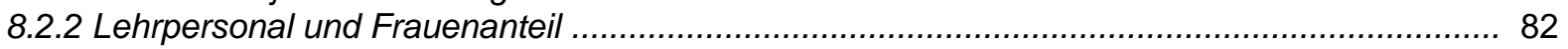

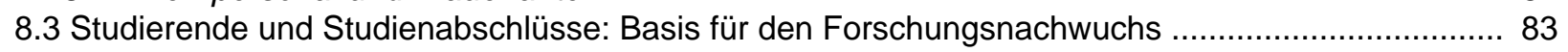

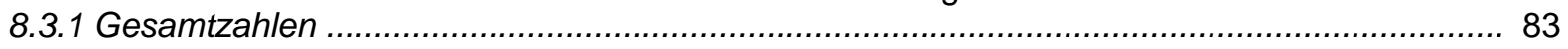

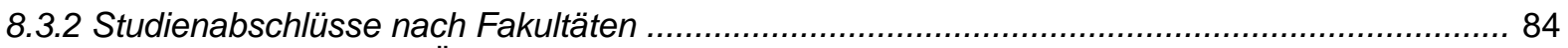

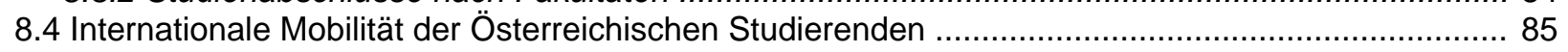

8.4.1 Förderungsprogramme für den Wissenschaftlichen Nachwuchs ....................................... 87

8.5 Wissenschaftliche Produktion der Universitäten ............................................................. 89

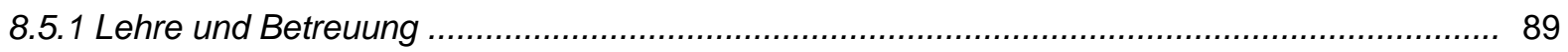

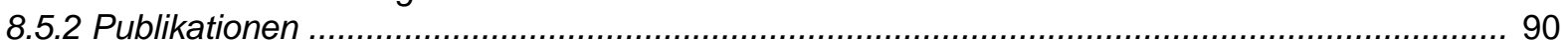

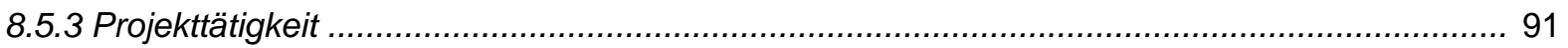

8.6 Internationaler Vergleich des Publikationsoutputs ............................................................... 92

8.7 Kooperationen zwischen Universitäten und Industrie in Österreich ............................................. 97

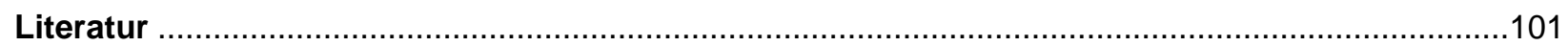

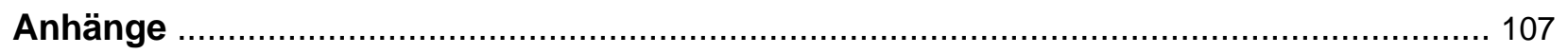

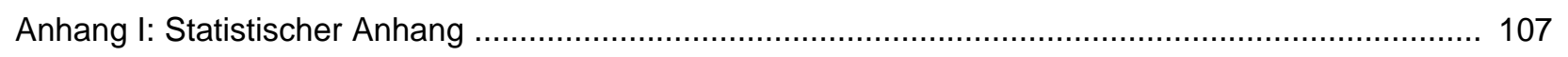

Anhang II: Szenarienrechnungen des Tip zur Erhöhung der F\&E-Quote .......................................... 165

Anhang III: Arbeitsaufträge aus der Erklärung der Bundesregierung vom 11. Juli 2000 ........................169

III/1: Bericht Der Projektgruppe „Hebung der Forschungsquote in der Wirtschaft“

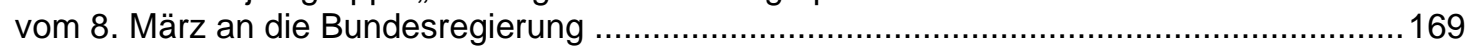

III/2: Statistische Erhebungen - Synchronisierung mit internationalen Organisationen ................ 173

III/3: Erweiterung der Bund/Bundesländer-Kooperation ...................................................... 175

III/4: Programm "Public Understanding of Science and Technology“ ....................................... 177 


\section{Einleitung}

Gemäss § 8 FOG 2000 hat das Bundesministerium für Bildung, Wissenschaft und Kultur (BMBWK) gemeinsam mit dem Bundesministerium für Verkehr, Innovation und Technologie (BMVIT) dem Nationalrat bis 1. Juni des Jahres einen Lagebericht über die aus Bundesmitteln geförderte Forschung, Technologie und Innovation in Österreich in Entsprechung des $\S 8$ Forschungsorganisationsgesetz vorzulegen.

Erstmals wird damit die bisher getrennte Berichterstattung über die Lage der Forschung (jährliche Forschungsberichte des BMBWK) und die technologische Leistungsfähigkeit Österreichs (tip-Technologieberichte 1997 und 1999) zu einem einheitlichen Dokument zusammengeführt. Damit tragen die für die Förderung der Forschung, Technologie und Innovation federführenden Ministerien BMBWK und BMVIT auch dem Umstand der wechselseitigen Verflechtung der wissenschaftlichen Grundlagenforschung einerseits und der anwendungsorientierten Technologieforschung andererseits sowie ihrer Förderung auch in Form eines einheitlichen Berichtswesens Rechnung; ein Lagebericht, der sich durch seinen analytisch-deskriptiven Stil von bisherigen Berichten unterscheidet.

Die Basis dieses Lageberichts bilden Beiträge der befassten Fachministerien (neben dem BMBWK und dem BMVIT auch das BMWA), von Statistik Austria sowie vor allem umfassende Analysen des "Technologie-, Informations- und Politikberatungsprogrammes" tip ${ }^{1}$, einer Initiative des BMVIT und des BMBWK. Schließlich wurden dankenswerterweise auch Daten vom österreichischen Universitätskuratorium zur Verfügung gestellt.

Zum Erreichen des Ziels der Bundesregierung, die Forschungsquote in Österreich bis zum Jahr 2002 auf 2,0\% und bis zum Jahr 2005 auf 2,5\% des Bruttoinlandsproduktes zu erhöhen ${ }^{2}$, sind große Anstrengungen und koordinierte Initiativen der verschiedenen Akteure der Forschungs- und Technologiepolitik nötig. Aus diesem Grund ist der Informationsbedarf entsprechend hoch. Dieser Bericht leistet als Teil einer umfassenderen Berichterstattung einen Beitrag zur Deckung dieses Informationsbedarfes.

Zudem hat der europäische Rat von Lissabon eine ehrgeizige Strategie des Wandels formuliert. Bis 2010 soll die Europäische Union „zum wettbewerbsfähigsten und dynamischsten wissensbasierten Wirtschaftsraum der Welt werden“ ${ }^{3}$. Voraussetzung für den Entwurf und die Umsetzung einer solchen Strategie sind umfassende Bestandsaufnahmen und Vergleiche (Benchmarking) der wissenschaftlichen und technologischen Leistungsfähigkeit der einzelnen Mitgliedsländer sowie der entsprechenden Politiken. Dieser Bericht versteht sich auch als ein österreichischer Beitrag zu diesen Bestandsaufnahmen und Benchmarking-Initiativen.

Der Forschungs- und Technologiebericht stützt sich auf die jeweils aktuellsten verfügbaren nationalen und internationalen Daten und orientiert sich am internationalen Stand der Innovationsforschung. Er

- stellt die österreichischen Forschungskapazitäten anhand grundlegender Indikatoren zur Forschungsfinanzierung und in F\&E tätigem Personal dar (Kapitel 2),

\footnotetext{
${ }^{1}$ tip, das Beratungsprogramm für die Forschungs- und Technologiepolitik des BMVIT und des BMBWK, ist ein Expertenverbund von Forscherlnnen aus dem WIFO (Leitung), JOANNEUM RESEARCH, ARCS und Technopolis. Die Analysen für diesen Lagebericht stammen von einer tip-Gruppe, bestehend aus W. Polt (Koordinator), B. Dachs, H. Gassler, A. Geyer, G. Hutschenreiter, N. Knoll, H. Leo, M. Paier, M. Peneder, S. Pohn-Weidinger, D. Schartinger, A. Schibany, G. Streicher.

${ }^{2}$ Erklärung der Bundesregierung zu aktuellen Fragen der Forschungs- und Technologiepolitik vom 11. Juli 2000

${ }^{3}$ Mitteilung der Kommission: „Das ganze Potential der Union ausschöpfen: Konsolidierung und Ergänzung der Lissabonner Strategie". KOM(2001) 79. Teil 1. Brüssel 2001)
} 
- unterzieht das österreichische Forschungs- und Technologiesystem einem internationalen Vergleich (Kapitel 3)

- beschreibt den Prozess der Internationalisierung des österreichischen Innovationssystems und analysiert die österreichische Beteiligung im 4. EU-Rahmenprogramm und in EUREKA (Kapitel 4),

- geht ein auf die aktuelle Debatte über effektive Instrumente der Forschungs- und Technologieförderung (Kapitel 5),

- beschreibt verschiedene Aspekte der technologischen Leistungsfähigkeit der österreichischen Unternehmen (Kapitel 6),

- stellt aktuelle Trends in den Sektoren der Informations- und Kommunikationstechnologie dar und diskutiert österreichische Aufholpotentiale (Kapitel 7)

- und gibt schließlich einen Überblick über das österreichische Universitätssystem und seinen Output (Kapitel 8) 


\section{Forschungskapazitäten in Österreich \\ Finanzierung von Forschung und experimenteller Entwicklung 2001 und Forschungs- personal 1998}

\subsection{F\&E-Ausgaben 2001 insgesamt (Tabellen 1 und la im statistischen Anhang)}

Für Forschung und experimentelle Entwicklung (F\&E) in Österreich werden 2001 gemäß der neuesten Schätzung von Statistik Austria (Ende April 2001) ${ }^{4}$ voraussichtlich insgesamt 54 Milliarden Schilling ausgegeben werden. Diese Ausgaben werden zu je ca. 40 Prozent von der öffentlichen Hand (Bund, Bundesländer, sonstige öffentliche Einrichtungen: 40,3 Prozent) und von der Wirtschaft (40,1 Prozent), zu 19,3 Prozent vom Ausland sowie zu 0,8 Prozent von sonstigen Quellen finanziert werden. Gegenüber 2000 wird die Gesamtsumme der österreichischen Forschungsausgaben um 6,4 Prozent steigen und damit 1,83 Prozent des Bruttoinlandsproduktes erreichen.

Demnach werden 2001 vom Bund 18,4 Milliarden Schilling (34,1 Prozent), von den Bundesländern 3,1 Milliarden Schilling (5,7 Prozent), von anderen öffentlichen Finanzierungsquellen (Gemeinden, Kammern, Sozialversicherungsträgern) 250 Millionen Schilling (0,5 Prozent) - somit zusammen von der öffentlichen Hand 21,8 Milliarden Schilling (40,3 Prozent) getragen werden, 21,6 Milliarden Schilling (40,1 Prozent) werden von der Wirtschaft, 10,4 Milliarden Schilling (19,3 Prozent) vom Ausland und nur 185 Millionen Schilling vom privaten gemeinnützigen Sektor finanziert werden.

Im Bundesbudget Forschung für 2001 sind unter dem VA-Ansatz 1/5182 12 für das F\&E-Offensivprogramm 7 Milliarden Schilling veranschlagt. Aufgrund der Statistik Austria im April vorliegenden Informationen (Rat für Forschung und Technologie, Bundesministerium für Finanzen) wurde jedoch von der Annahme ausgegangen, dass von diesen 7 Milliarden Schilling im Jahre 2001 nur ca. 2 Milliarden Schilling ausgegeben werden und die Inanspruchnahme der restlichen 5 Milliarden Schilling für F\&E-Ausgaben der Folgejahre (2002 und 2003) vorgesehen ist. In die vorliegende F\&E-AusgabenSchätzung von Statistik Austria sind daher in Bezug auf den Finanzierungsanteil des Bundes - abweichend von der Beilage T/Teil b - nur jene 2 Milliarden Schilling, welche im Jahre 2001 der Forschung zufließen sollen, aufgenommen worden. In die Schätzung für das Jahr 2000 sind auch die restlichen Rücklagen aus den „Technologiemilliarden“ der Jahre 1997 und 1998 in Höhe von ca. 839 Millionen Schilling, die im Jahre 2000 für Forschungsvorhaben verwendet worden sind, aufgenommen worden.

Die erstmals von Statistik Österreich durchgeführte F\&E-Erhebung im firmeneigenen Bereich über das Berichtsjahr $1998^{5}$, von welcher mit einem detaillierteren Frageprogramm nicht nur der produzierende Bereich, sondern erstmals auch der Dienstleistungssektor erfasst wurde, erbrachte hinsichtlich der Finanzierungsstruktur der Forschung in Österreich neue Resultate, die selbstverständlich in die Globalschätzung 2001 Eingang fanden: Danach wird die Forschungstätigkeit in Österreich auch im Jahre 2001 zu einem erheblichen Teil (19,8 Prozent) vom Ausland finanziert. Diese Mittel stammen zum überwiegenden Teil von mit heimischen Unternehmen verbundenen europäischen Unternehmen, die Österreich zum Forschungsstandort gewählt haben. Bis zu einem gewissen Grad dürfte dies auch schon vor 1993 zutreffend gewesen sein, im Rahmen der bisherigen F\&E-Erhebungen im industriellen Bereich (zuletzt durch die Wirtschaftskammer Österreich über das Berichtsjahr 1993) konnte dies jedoch nicht verifiziert werden. Doch hat die Europäische Integration zweifellos wesentlich dazu bei-

\footnotetext{
${ }^{4}$ Die Gesamtschätzung 2001 und Ergebnisse der F\&E-Erhebung 1998 durch Statistik Austria zum Forschungspersonal lagen erst Anfang Mai 2001 vor. Die Daten einiger Abschnitte basieren daher auf dem Stand des Jahres 2000.

${ }^{5}$ Vgl. „Forschung und experimentelle Entwicklung (F\&E) im firmeneigenen Bereich 1998“. In: Statistische Nachrichten, Heft 2/2001, S. $89-103$.
} 
getragen, dass internationale Konzerne die Standortvorteile Österreichs nützen und in verstärktem Maße ihre Forschungstätigkeit in Österreich durchführen.

In der Finanzierung durch das Ausland sind definitionsgemäß auch die anteiligen Rückflüsse an österreichische Forschungseinrichtungen im Rahmen der jeweiligen EU-Rahmenprogramme für Forschung, technologische Entwicklung und Demonstration enthalten. Mit Stand April 2001 waren aus dem derzeit laufenden 5. Rahmenprogramm für Forschung, technologische Entwicklung und Demonstration insgesamt rund 1,6 Mrd. S bewilligt, von denen 2001 voraussichtlich ca. 450 Millionen Schilling an österreichische Forschungseinrichtungen zurückfließen werden.

Die Gesamtsumme der österreichischen Forschungsausgaben 2001 wird um 70,3 Prozent über dem Niveau von 1993 und um 14,2 Prozent über 1998 liegen. Die Forschungsausgaben wuchsen (trotz einer deutlichen Verlangsamung des Wachstums Ende der Neunziger Jahre) demnach um einiges schneller als das Bruttoinlandsprodukt (BIP), das nominell im Zeitraum 1993 bis 2001 um 36,2 Prozent und im Zeitraum 1998 bis 2001 um 12,5 Prozent gestiegen ist.

Eine Übersicht über die gesamten F\&E-Ausgaben 2001 enthält die Tabelle 1 im statistischen Anhang, in welcher die Finanzierung der in Österreich durchgeführten Forschung und experimentellen Entwicklung dargestellt ist. Als zusätzliche Information weist Tabelle 1a im Anhang die Finanzierungsanteile der einzelnen Sektoren in Prozent des Bruttoinlandsprodukts aus.

\subsection{F\& E-Ausgaben des Bundes 2001 (Tabellen 2 bis 8 im statistischen Anhang)}

Die Ausgaben des Bundes für in Österreich durchgeführte F\&E werden 2001 rund 18,4 Milliarden Schilling erreichen, sie liegen damit um rund 12,4 Prozent über dem Vorjahresniveau, um 18,5 Prozent über dem Niveau von 1998, und um 40,0 Prozent über dem Niveau von 1993 (s. Tabelle 1 im Anhang).

Gemäß der zugrundeliegenden Methodik handelt es sich bei den in Tabelle 1 ausgewiesenen Ausgaben des Bundes für in Österreich durchgeführte F\&E für die Jahre 2000 und 2001 um Bundesvoranschlagswerte. Für das Jahr 2001 fanden in der Schätzung des Finanzierungsanteiles des Bundes durch Statistik Austria jedoch (abweichend von den im Bundesbudget Forschung für 2001 unter dem VA-Ansatz 1/5182 12 für das F\&E-Offensivprogramm veranschlagten 7 Milliarden Schilling) nur 2 Milliarden Schilling Berücksichtigung, da der Rest von 5 Milliarden Schilling für F\&E-Finanzierungen des Bundes in den beiden Folgejahren vorgesehen ist. Die volle Berücksichtigung im Jahre 2001 würde die Schätzung der Finanzierungsanteile des Bundes sowohl für 2001 als auch für die Folgejahre stark verzerren. Zusätzlich zu den Ausgaben für in Österreich durchgeführte F\&E wird der Bund im Jahre 2001 Beitragszahlungen, in Höhe von 602,6 Millionen Schilling an internationale Organisationen, die Forschung und Forschungsförderung als Ziel haben, leisten.

Die gesamten Ausgaben des Bundes für Forschung und Forschungsförderung (einschließlich der forschungswirksamen Anteile der Beitragszahlungen an internationale Organisationen) sind in der "Beilage T des Amtsbehelfes zum Bundesfinanzgesetz, Teil a und Teil b", zusammengefasst (siehe Tabelle 3 im Anhang); die Werte aus dem Bundesrechnungsabschluss 1999 enthalten auch die im Jahre 1999 eingesetzten forschungswirksamen Mittel aus den „Technologie- und Exportoffensiven 1998 und 1999“ (rund 643 Millionen Schilling).

In Tabelle 2 im statistischen Anhang werden die Ausgaben des Bundes für Forschung und Forschungsförderung 2001 gemäss Bundesfinanzgesetz 2001 (Beilage T) unter Einschluss der im Budgetkapitel 51 rücklagefähig veranschlagten Mittel der „Forschungs- und Technologieoffensive“ in Höhe von 7 Milliarden Schilling dargestellt. Das Gesamtvolumen der veranschlagten Mittel erreicht damit rund 24 Milliarden Schilling. Da die Zuweisung der im Jahr 2001 zur Verfügung stehenden Mittel aus 
der "Forschungs- und Technologieoffensive" an die jeweils zuständigen Ressorts unter Berücksichtigung der Empfehlungen des Rates für Forschung und Technologieentwicklung in mehreren Schritten erfolgt, kann über die tatsächliche Gesamthöhe der Bundesausgaben 2001 und deren Proportionen nach Ressorts erst im folgenden Jahr berichtet werden.

Für die Bundesrechnungsabschlussdaten 1999 und die Voranschlagsdaten für 2000 und 2001 wurden von Statistik Austria die Ausgaben des Bundes für Forschung und Forschungsförderung (einschließlich der forschungswirksamen Anteile an den Beitragszahlungen an internationale Organisationen) nach sozio-ökonomischen Zielsetzungen funktionell aufgegliedert (siehe Tabelle 4 im statistischen Anhang).

Ohne Berücksichtigung der Mittel der „Forschungs- und Technologieoffensive“, deren Vergabe und Aufteilung auf die Ressorts in den Jahren 2001 - 2003 aufgrund von Empfehlungen des Rates für Forschung und Technologieentwicklung erfolgt, ergeben sich für das Jahr 2001 folgende Schwerpunkte für die Förderung von F\&E durch den Bund (siehe Tabelle 8 im statistischen Anhang):

35,5 Prozent: Förderung der allgemeinen Erweiterung des Wissens

22,9 Prozent: Förderung des Gesundheitswesens

14,4 Prozent: Förderung von Handel, Gewerbe und Industrie

6,9 Prozent: Förderung der sozialen und sozio-ökonomischen Entwicklung

5,1 Prozent: Förderung der Erforschung der Erde, der Meere, der Atmosphäre und des Weltraumes

4,3 Prozent: Förderung der Land- und Forstwirtschaft

3,7 Prozent: Förderung des Umweltschutzes

2,7 Prozent: Förderung des Transport-, Verkehrs- und Nachrichtenwesens

Gegenüber dem Jahr 2000 sind die stärksten Zuwächse in den Kategorien „Förderung von Handel, Gewerbe und Industrie“ (+10,3 Prozent), „Förderung der Landesverteidigung“ (+17,9 Prozent) und „Förderung des Gesundheitswesens“ (+9,0 Prozent) zu beobachten. Die mit einem Anteil von 35,5 Prozent größte Kategorie „Förderung der allgemeinen Erweiterung des Wissens“ weist einen geringen Rückgang (-0,5 Prozent) gegenüber 2000 auf.

Für 1998, 1999, 2000 und 2001 liegen Aufgliederungen der Jahreswerte nach sozio-ökonomischen Zielsetzungen auch nach Ressorts vor (siehe Tabellen 5, 6, 7, und 8 im statistischen Anhang).

\subsubsection{Auswertungen der "Faktendokumentation 1999" (Tabellen 9 bis 14 im statistischen Anhang)}

Seit 1976 werden die Forschungsförderungen und Forschungsaufträge der Bundesdienststellen im Bundesministerium für Bildung, Wissenschaft und Kultur in einer Datenbank erfasst. Statistik Österreich wertet diese Datenbank jährlich nach verschiedenen Kriterien aus. Für das Jahr 1999 liegen folgende Auswertungen vor:

a) nach Förderungsempfängern bzw. Auftragnehmern,

b) nach sozio-ökonomischen Zielsetzungen sowie

c) nach Wissenschaftszweigen. 
Wie für die vergangenen Jahre wurden auch für 1999 jeweils gesonderte Auswertungen unter Einschluss der „großen" d.h. institutionellen Globalförderungen (Forschungsförderungsfonds, Ludwig Boltzmann-Gesellschaft, Österreichische Akademie der Wissenschaften, Österreichisches Forschungszentrum Seibersdorf) erstellt. Die Auswertungen "ohne große Globalförderungen" umfassen die an Institutionen bzw. Einzelpersonen erteilten Forschungsaufträge und Expertengutachten sowie Basisförderungen an kleine Forschungseinrichtungen. (Die in der Faktendokumentation 1999 nicht enthaltene Globalförderung seitens des BMWA an den FFF wurde gemäss Bundesrechnungsabschluss 1999 inkludiert). Unter Berücksichtigung dieser Mittel entfielen 1999 mehr als drei Viertel (76,8 Prozent) der Gesamtsumme der Forschungsförderungen und Forschungsaufträge auf die „großen" (institutionellen) Globalförderungen.

Wesentliche Ergebnisse dieser Auswertungen sind:

Von den gesamten erfassten Mitteln (d.h. unter Einschluss der „großen" Globalförderungen) wurden 73,7 Prozent vom BMWV, 22,7 Prozent vom BMWA sowie der Rest (3,7 Prozent) von den anderen Ressorts ausbezahlt.

Ohne die "großen" Globalförderungen ergibt sich, dass 81,4 Prozent der Mittel vom BMWV, 5,4 Prozent vom BMLF, 4,4 Prozent vom BMUJF, 2,8 Prozent vom BMWA, 2,2 Prozent vom BKA und der Rest (3,9 Prozent) von den anderen Ressorts vergeben wurden.

Die Auswertung der Gesamtförderung nach Förderungsempfängern bzw. Auftragnehmern (siehe Tabelle 9 im statistischen Anhang) ergab, dass - abgesehen von den über die beiden Forschungsförderungsfonds vergebenen Förderungen (FWF: 25,9 Prozent; FFF: 22,3 Prozent) 20,5 Prozent der Teilbeträge 1999 an Förderungsempfänger bzw. Auftragnehmer im Hochschulsektor, 14,3 Prozent an solche im Unternehmenssektor, und 13,1 Prozent an Einrichtungen im Sektor Staat vergeben wurden. Unter Einbeziehung der über den FFF vergebenen Förderungen gingen 36,6 Prozent der im Jahre 1999 ausbezahlten Mittel an den Unternehmenssektor.

Lässt man die "großen" Globalförderungen außer Betracht (siehe Tabelle $10 \mathrm{im}$ Anhang), so wurden 35,8 Prozent der Forschungsaufträge und Förderungen 1999 an Vertragspartner im Hochschulsektor, 28,3 Prozent an solche im Sektor Staat, 16,1 Prozent an Auftragnehmer im Unternehmenssektor und 15,4 Prozent an den privaten gemeinnützigen Sektor vergeben.

Bei der Auswertung nach sozio-ökonomischen Zielsetzungen unter Einschluss der "großen" Globalförderungen (siehe Tabelle $11 \mathrm{im}$ Anhang) ergab sich, dass 1999 der „Förderung der allgemeinen Erweiterung des Wissens" mit 31,9 Prozent das größte Gewicht zukam. 24,0 Prozent der Förderungsmittel waren der Kategorie „Förderung von Handel, Gewerbe und Industrie" zurechenbar. Werden die "großen" Globalförderungen außer Betracht gelassen (siehe Tabelle 12 im Anhang), so entfielen auf die „Förderung der allgemeinen Erweiterung des Wissens" 31,7 Prozent der gesamten Förderungsmittel, auf die „Förderung der sozialen und sozio-ökonomischen Entwicklung" 22,8 Prozent und auf die „Förderung von Handel, Gewerbe und Industrie" 8,7 Prozent.

Die Auswertung nach Wissenschaftszweigen unter Einschluss der "großen" Globalförderungen (siehe Tabelle 13 im Anhang) ergab, dass 1999 die größten Anteile an den Förderungsbeträgen auf die Technischen Wissenschaften (33,4 Prozent) und die Naturwissenschaften (31,2 Prozent) entfielen. Unter Ausklammerung der "großen" Globalförderungen (siehe Tabelle 14) entfielen auf die Sozialwissenschaften 35,0 Prozent, auf die Naturwissenschaften 27,9 Prozent und auf die Technischen Wissenschaften 17,2 Prozent der 1999 ausbezahlten Förderungsbeträge. 


\subsection{F\&E-Ausgaben der Bundesländer 2001}

Die F\&E-Ausgaben der Bundesländer werden von Statistik Austria unter Heranziehung der Meldungen der Ämter der Landesregierungen für 2001 mit 3,064 Mrd. S geschätzt (die F\&E-Ausgaben der Landeskrankenanstalten sind in diese Schätzung einbezogen). Sie liegen damit um rund 4,4 Prozent über dem Niveau von 2000 und um 71,7 Prozent über dem Erhebungsergebnis von 1993 (siehe Tabelle 1 im Anhang).

\subsection{Forschung und Entwicklung im Unternehmenssektor}

Abbildung 2-1: Forschungsintensität und Wertschöpfung nach Branchen des österreichischen Unternehmenssektors, 1998

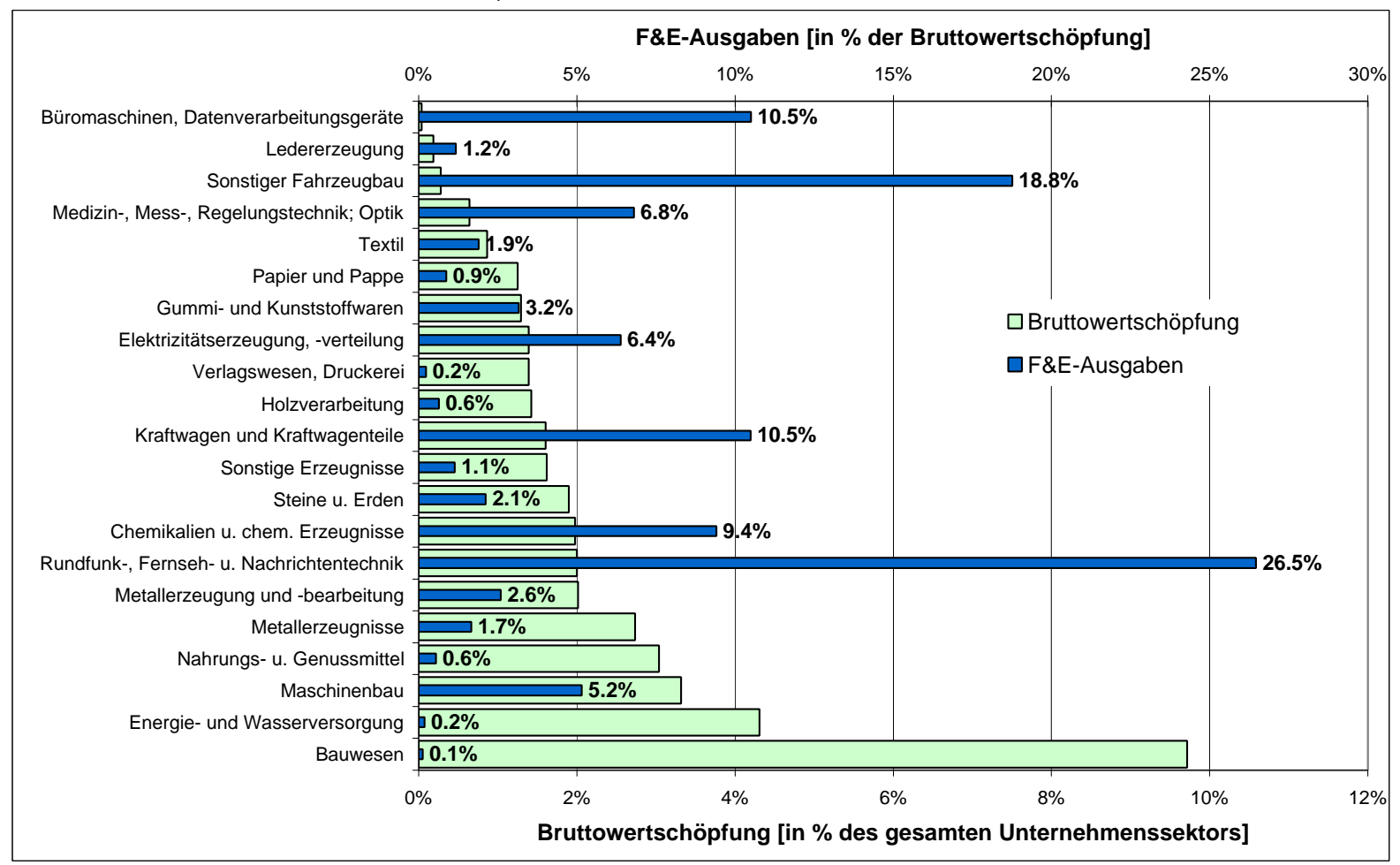

Quelle: Statistik Austria (2001)

Für Österreich kann der Anteil des Unternehmenssektors an der Durchführung von F\&E auf Branchenebene weiter aufgeschlüsselt werden. Im produzierenden Sektor weisen die Zahlen für 1998 zur

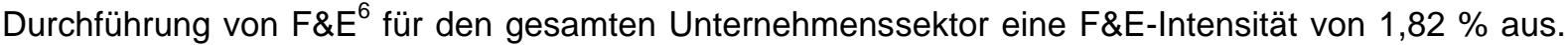
Die F\&E-Aufwendungen bezogen auf die Bruttowertschöpfung in der Sachgüterproduktion, auf die 84 $\%$ der gesamten Unternehmens-F\&E entfällt, betrug 1998 4,78\%. Im Vergleich dazu hatte die Bereichszählung der Statistik Austria für 1995 eine Intensität von 4,2\% angegeben, was auf eine leichte Steigerung der Forschungsintensität in der österreichischen Sachgüterproduktion in den letzten Jahren hindeutet.

Wie der internationale Vergleich zeigt, sind hohe gesamtwirtschaftliche F\&E-Quoten vor allem auf hohe F\&E-Aufwendungen des jeweiligen Unternehmenssektors zurückzuführen. Jedoch nicht alle Branchen sind im selben Maße forschungsintensiv. Einzelne besonders forschungsintensive Branchen können daher - bei entsprechend hohem Anteil an der Wirtschaft eines Landes - die Forschungsquote maßgeblich beeinflussen.

\footnotetext{
${ }^{6}$ Erhebung über Forschung und experimentelle Entwicklung 1998, Statistik Austria 2001.
} 
Auf Branchenebene (siehe Abbildung 2-1) sind besonders forschungsintensive Branchen die Rundfunk-, Fernseh- und Nachrichtentechnik, sonstiger Fahrzeugbau, Kraftfahrzeuge und BüromaschinenComputer, sowie Chemie (inkl. pharmazeutische Industrie). Wie der österreichische Technologiebericht 1999 bereits zeigte, ist der im Vergleich zur OECD geringe Anteil einiger dieser Branchen mit ein Grund für den Rückstand der gesamtwirtschaftlichen F\&E-Quote Österreichs zum OECDDurchschnitt. An diesem Befund hat sich auch in den letzten Jahren wenig geändert. Zwar zeigen einige der forschungsintensiven Branchen höhere Wachstumsraten als die Gesamtwirtschaft und gewinnen dadurch anteilsmäßig. Diese Zuwächse sind jedoch zu gering, um die gesamtwirtschaftliche Forschungsquote wesentlich zu heben.

\section{Abbildung 2-2 : Extern vergebene Forschung und Entwicklung österreichischer Unternehmen im Inland,} 1998 in 1.000 ATS

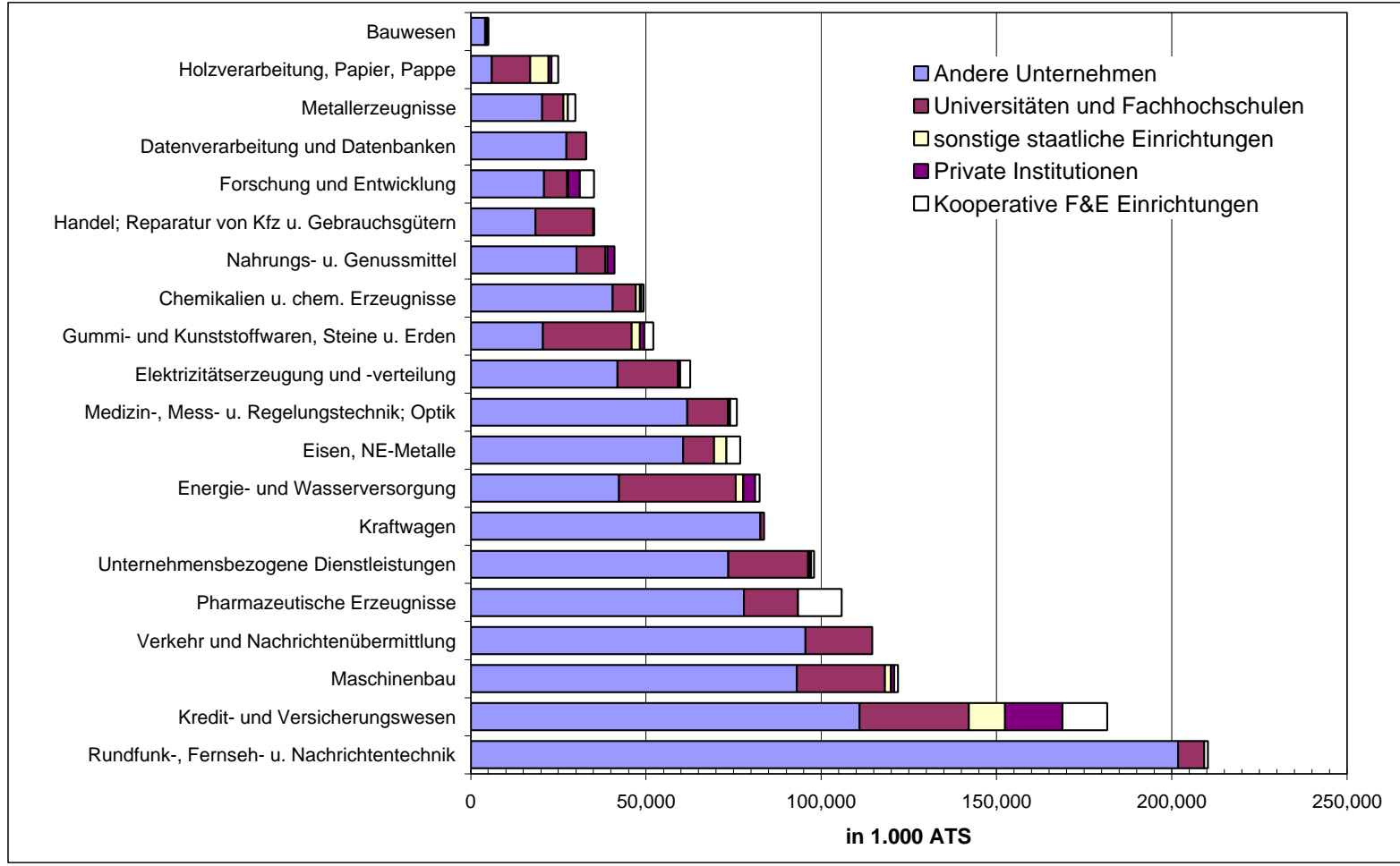

Quelle: Statistik Austria (2001)

Innovationen sind zunehmend das Ergebnis von Kooperationen und Wissensflüssen in Netzwerken aus Unternehmen und akademischen Forschungseinrichtungen. Ein Teil dieser Verflechtungen kann durch die Finanzierungsströme für extern vergebene Forschung und Entwicklung dargestellt werden. Finanzierungsquelle ist in diesem Fall der Unternehmenssektor, der 1998 1,6 Mrd. ATS für externe F\&E im Inland aufwendete. Wie Abbildung 2-2 zeigt, geht der größte Teil dieser Mittel wiederum an andere Unternehmen, wobei diese Art von Kooperationen in allen Branchen von ihrem finanziellen Umfang her die bedeutendste ist. Verflechtungen zwischen österreichischen Unternehmen und Universitäten sind von ihrem finanziellen Umfang her dagegen weit weniger bedeutend. Nur etwa $20 \%$ der an Dritte vergebenen Forschungsmittel kommen Universitäten zugute, wobei auch forschungsintensive Branchen wie die Rundfunk- Fernseh- und Nachrichtentechnik oder die Pharmaindustrie relativ zurückhaltend sind. Die daraus ersichtliche mangelnde Verflechtung des österreichischen Unternehmenssektors mit anderen Teilen des nationalen Innovationssystems, vor allem mit den Universitäten, wurde bereits in früheren Technologieberichten angesprochen. Relativ hoch ist hingegen die extern vergebene Forschungsleistung des Dienstleistungssektors. Die betreffenden Unternehmen erreichen mit 507 Mio. ATS externer F\&E etwa die Hälfte des Vergleichswerts der Sachgüter- 
erzeugung bei wesentlich geringerer Forschungsintensität. Dies deutet auf einen hohen komplementären Charakter von extern vergebener F\&E für die Unternehmen hin, die im Regelfall keine eigenen F\&E-Abteilungen betreiben. 


\subsubsection{Forschungs- und Entwicklungsaktivitäten des Dienstleistungssektors}

Abbildung 2-3: Anteil des Dienstleistungssektors an den F\&E-Ausgaben der Unternehmen, 1995

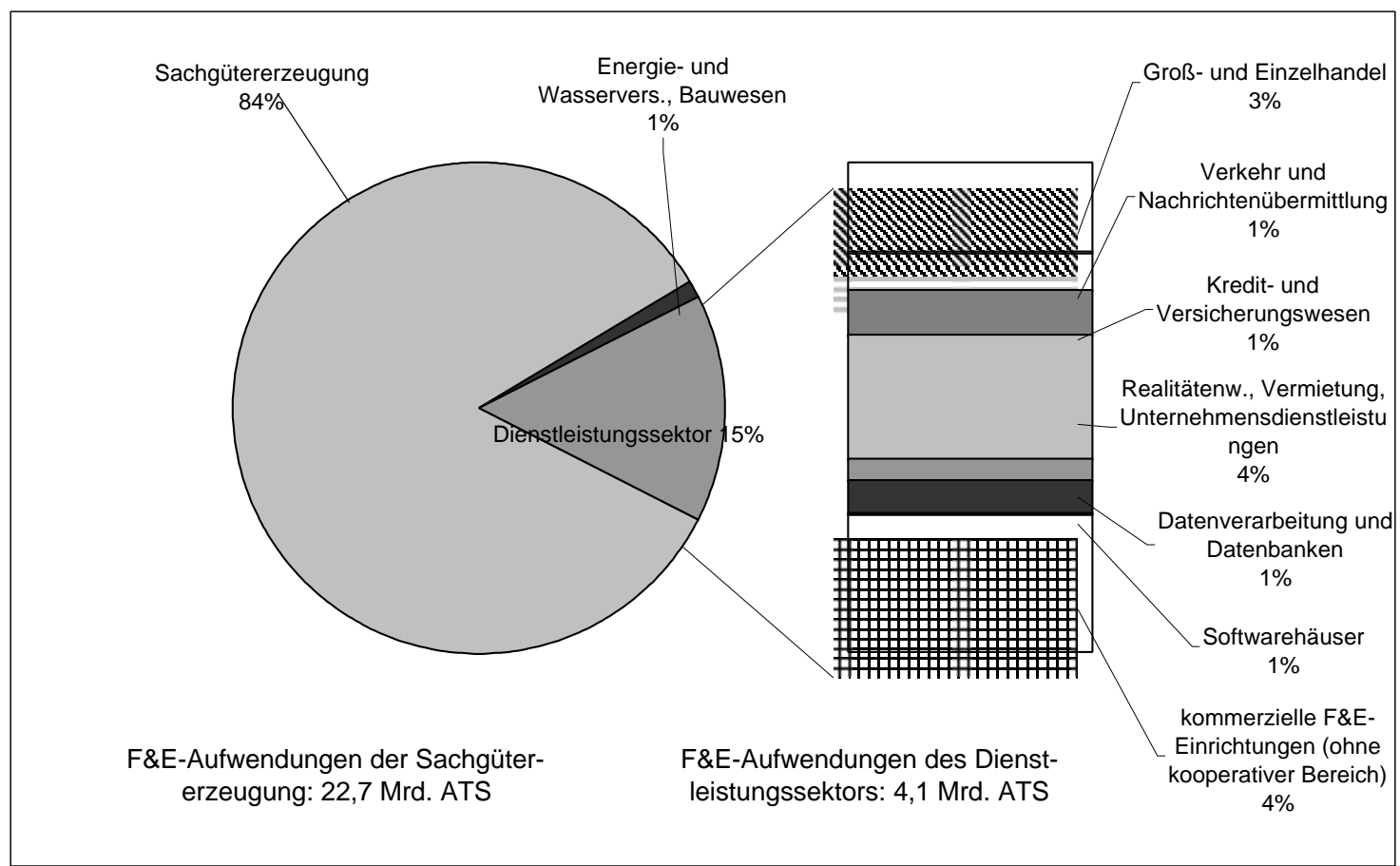

Quelle: Statistik Austria (2001)

Abbildung 2-4: Anteil forschender Unternehmen im österreichischen Dienstleistungssektor, 1994-96

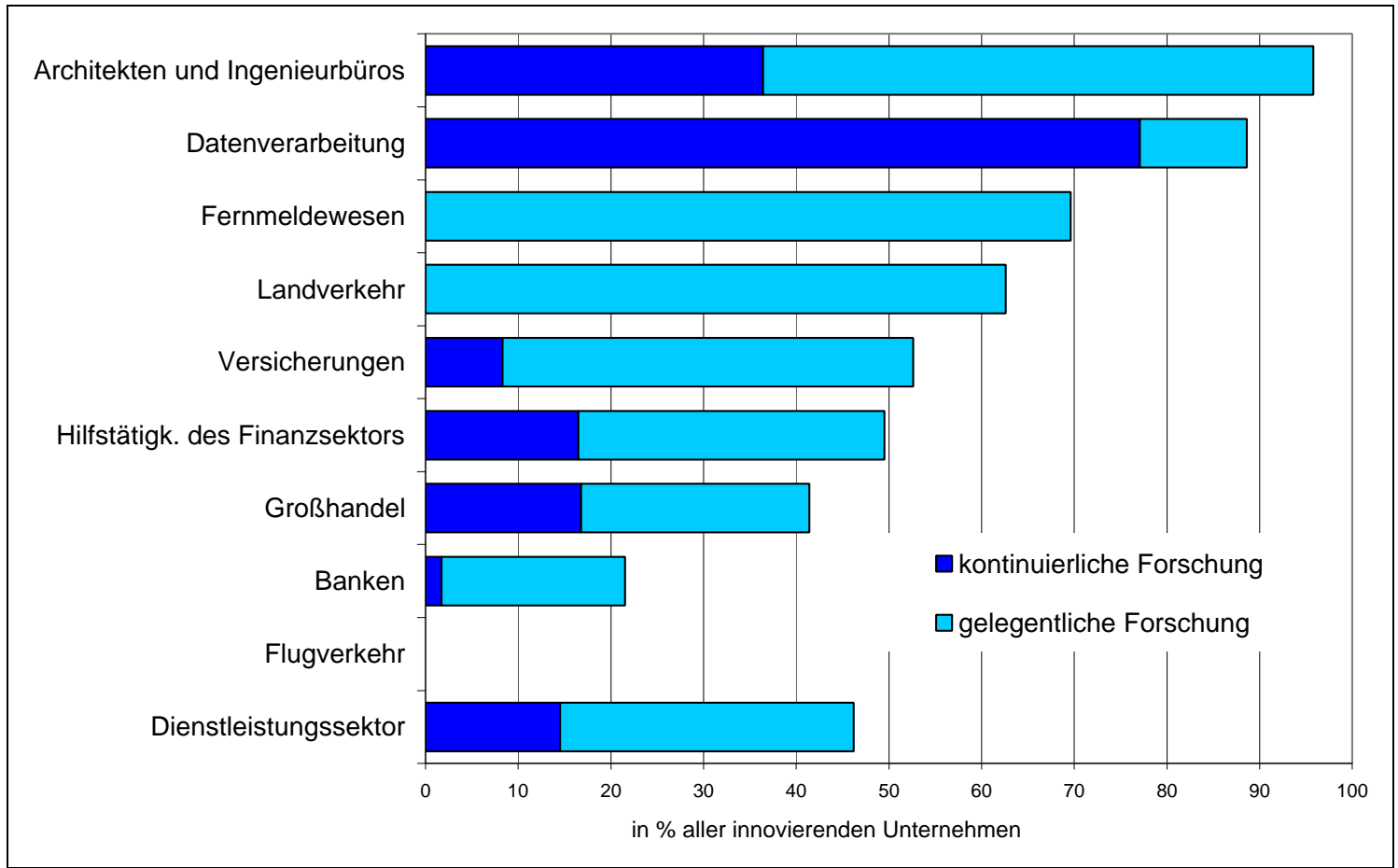

Quelle: Community Innovation Survey, WIFO. 
Die Bewertung des Dienstleistungssektors in technologie- und innovationspolitischer Hinsicht hat sich während der letzten Jahre gewandelt. Technologieorientierte, dynamisch wachsende Teilsektoren wie Telekommunikation und Datenverarbeitung haben das tradierte Bild der Dienstleistungen als arbeitsintensive Bereiche der Wirtschaft mit langsamer Produktivitätsentwicklung nachhaltig verändert. Die erstmalige Berücksichtigung der entsprechenden Sektoren in der offiziellen Statistik entspricht diesem Einstellungswandel. Demnach betrugen 1998 die internen Forschungs- und Entwicklungsaufwendungen österreichischer Dienstleistungsunternehmen 4,07 Mrd. ATS oder $15 \%$ der F\&EAusgaben des österreichischen Unternehmenssektors, womit der Anteil dem OECD-Durchschnitt für das Jahr 1995 entspricht (vergleiche OECD, 2000).

Die Expansion von F\&E im Dienstleistungssektor, die internationale Statistiken seit einigen Jahren nachzeichnen, kann nicht auf eine einzige Ursache zurückgeführt werden. In vielen Fällen steht sie in enger Verbindung mit der wachsenden Arbeitsteilung und Kooperation im Innovationsprozess. Wissensintensive Dienstleister etablieren sich dabei immer mehr als Vorleister für Innovationen in Sachgüterproduktion oder anderen Dienstleistungsbranchen. Die engen Beziehungen und Netzwerke zwischen den spezialisierten Wissenszulieferern und innovierenden Unternehmen sind Zeichen eines zunehmend komplexen Innovationsprozesses. Die bedeutendsten F\&E-bezogenen Sektoren in dieser Gruppe sind Unternehmensberater und Ingenieurbüros (ÖNACE 74; 1 Mrd. ATS F\&EAufwendungen), Softwareunternehmen (ÖNACE 72; 455 Mio. ATS) und kommerzielle F\&EEinrichtungen $^{7}$ (ÖNACE 73; 1,14 Mrd. ATS). Vielfach bestehen über Kooperationen hinaus auch organisatorische Verflechtungen, die beispielsweise das Ergebnis von Ausgliederungen (etwa im Pharmabereich) und spin-offs sind.

Forschung und Entwicklung findet sich in steigendem Maß aber auch in anderen, konsumnäheren Branchen des Dienstleistungssektors wie dem Handel (NACE 50-52; 763 Mio. ATS), Verkehr und Telekommunikation (NACE 60-64; 315 Mio. ATS) oder dem Finanzsektor (NACE 65-67; 374 Mio. ATS) als Grundlage neuer Produkte oder Prozesse. Der Zusammenhang von F\&E und Innovation ist im Dienstleistungssektor jedoch wesentlich schwächer ausgeprägt als in der Industrie. Weniger als die Hälfte der Unternehmen (Industrie: 60 \%), die zwischen 1994 und 1996 Innovationen getätigt haben, haben auch geforscht (vergleiche Abbildung 2-3). Der Anteil der Unternehmen, die Patentansuchen stellten, ist noch geringer (Dachs, Leo 1999). Forschungsaktivitäten in Dienstleistungsunternehmen sind informeller und anlassbezogener organisiert und folgen oft nicht kontinuierlich. Es existieren nur in den seltensten Fällen eigene F\&E-Abteilungen, Forschung wird hauptsächlich als Begleitforschung betrieben (Sundbo, Gallouj 1998). Die Forschungsintensität im Dienstleistungssektor, gemessen in Ausgaben pro Mitarbeiter oder Wertschöpfungseinheit, ist daher wesentlich geringer als in der Industrie.

Da ein bedeutender Teil der im Dienstleistungssektor eingesetzten Technologien nicht in den Unternehmen selbst entwickelt wird, sondern aus der Industrie stammt, ist Technologiediffusion, in Form von materiellen und immateriellen Investitionsgütern (wie z. B. Software) für Dienstleistungsinnovationen wesentlich bedeutender als eigene Forschung. Eine frühere tip-Studie (Hutschenreiter, Kaniovski 1999) hat gezeigt, dass der Dienstleistungssektor bedeutend mehr Technologie in Form von Investitionsgütern aufnimmt als die Industrie. Innovationsprozesse im Dienstleistungssektor sind daher in vielerlei Hinsicht durch enge Lieferanten-Kunden-Beziehungen gekennzeichnet (Hipp, 2000), wobei auch die Abnehmer beträchtliche technologische Kompetenzen aufbauen. Von besonderer Bedeutung sind in diesem Zusammenhang die modernen Informations- und Kommunikationstechnologien, die in

\footnotetext{
${ }^{7}$ Dem ÖNACE 73, Forschung und Entwicklung, gehören eigentlich auch ARCS, Arsenal Research und Joanneum Research an, in der zitierten Erhebung sind diese Einrichtungen jedoch nicht im Unternehmenssektor berücksichtigt, sondern dem „kooperativen Bereich“" zugeordnet, vergleiche Statistik Austria 2001.
} 
vielen Branchen wie dem Finanzsektor oder dem Handel große technologische Möglichkeiten für Innovationen eröffnen. Die IKT-Investitionen des Finanzsektors und der Nachrichtenübermittlung übersteigen jene der Industrie um das Doppelte. Forschung im Dienstleistungssektor steht deshalb vermutlich oft im Zusammenhang mit der Adaption und Weiterentwicklung von externen Technologien. Ein wesentlicher Teil der F\&E, innerhalb, aber auch außerhalb des Sektors Datenverarbeitung, ist dabei IKT-bezogen.

\subsection{Gesamtösterreichischer Personaleinsatz für F\&E 1998 (Tabellen 19 und 20 im statistischen Anhang)}

Die Tabelle 19 zeigt in einer Zusammenfassung sämtlicher Erhebungsbereiche die Verteilung des Personaleinsatzes für F\&E auf die Durchführungssektoren in Kopfzahlen und in Vollzeitäquivalenten für F\&E. Tabelle 20 weist darüber hinaus auch die Verteilung der Personalressourcen nach dem Geschlecht aus.

Das Gesamtergebnis der Erhebungen 1998 von Statistik Austria über den Personaleinsatz in F\&E ergibt, dass dieser dem Einsatz von 31308 ganzjährig Vollbeschäftigten entsprach (siehe Tabelle 19). Von diesen 31308 Vollzeitäquivalenten für F\&E waren 27,7 Prozent im Hochschulsektor (1993: 29,2 Prozent), 6,7 Prozent im Sektor Staat (1993: 8,6 Prozent), 0,5 Prozent im privaten gemeinnützigen Sektor (1993: 0,4 Prozent) , 6,0 Prozent kooperativen Bereich und im Bereich der Ziviltechniker (1993: 5,5 Prozent) sowie 59,2 Prozent im Bereich der firmeneigenen Forschung (1993: 56,3 Prozent) eingesetzt. Auf den Unternehmenssektor zusammen entfallen somit 65,1 Prozent der Beschäftigten für F\&E (1993: 61,8 Prozent).

In der Gliederung nach funktionellen Beschäftigtenkategorien entfallen 59,8 Prozent auf das wissenschaftliche Personal (1993: 52,4 Prozent; 1989: 38,1 Prozent), 25,3 Prozent auf höherqualifiziertes nichtwissenschaftliches Personal - Techniker, Maturanten und gleichwertige Kräfte (1993: 26,2 Prozent; 1989: 36,5 Prozent) sowie 14,9 Prozent auf sonstiges Hilfspersonal (1993: 21,4 Prozent; 1989: 25,4 Prozent). Damit hat sich der schon 1993 beobachtete Trend hin zu besser ausgebildetem Personal auch 1998 weiter fortgesetzt.

30,4 Prozent der in Österreich in F\&E tätigen WissenschafterInnen sind an Universitäten (30,1 Prozent) und Universitäten der Künste (0,3 Prozent) beschäftigt; 5,1 Prozent entfallen auf sonstige staatliche Einrichtungen, 0,5 Prozent auf den privaten gemeinnützigen Sektor und 62,6 Prozent auf den Unternehmenssektor (darunter entfallen auf den firmeneigenen Bereich 58,4 Prozent).

\subsubsection{Beschäftigte in F\&E nach Wissenschaftszweigen 1989, 1993 und 1998}

Tabelle 26 im statistischen Anhang zeigt in einer Zusammenfassung aller Erhebungsbereiche - unter Ausklammerung des firmeneigenen Bereichs, in dem eine solche Zuordnung nicht zielführend wäre die Beschäftigten in F\&E nach den Wissenschaftszweigen, denen deren F\&E-Aktivitäten zugeordnet werden können. Die Verteilung hat sich seit 1989 im wesentlichen nicht geändert: Rund drei Viertel der eingesetzten Ressourcen entfallen auf naturwissenschaftlich-technische Forschung unter Einschluss von Humanmedizin sowie von Land- und Forstwirtschaft und Veterinärmedizin, rund ein Viertel auf sozialwissenschaftliche und geisteswissenschaftliche Forschung, wobei bereits seit $1985 \mathrm{zwi}-$ schen den Erhebungsjahren eine leichte und kontinuierliche Verschiebung im Verhältnis der Wissenschaftszweige zueinander um je 1 Prozentpunkt zugunsten der naturwissenschaftlich-technischen Forschung zu beobachten ist. 


\subsubsection{Beschäftigte in F\&E nach Wirtschaftszweigen 1998}

Tabelle 27 im Anhang zeigt für den seit der F\&E-Erhebung 1998 ebenfalls von Statistik Austria erfassten firmeneigenen Bereich die Verteilung des Personaleinsatzes und der Ausgaben für F\&E nach den Wirtschaftszweigen, denen die F\&E-Aktivitäten dienen. Für die Zuordnung wurde - in Übereinstimmung mit den modifizierten Richtlinien des 1993 revidierten Frascati-Manuals - die Systematik der Wirtschaftsklassen ISIC, Rev. 3, welche praktisch mit der NACE, Rev. 1, bzw. der ÖNACE identisch ist, herangezogen. Die Auswertung zeigt, dass auf den erstmals erfassten Bereich der Dienstleistungen immerhin ca. 15 Prozent der F\&E-Ressourcen entfallen (Beschäftigte in F\&E: 15,6 Prozent; Ausgaben für F\&E: 15,2 Prozent).

\subsubsection{Personelle Kapazitäten der universitären Forschung 1998 (Tabellen 16, 17 und 18 im statistischen Anhang)}

Gesamtösterreichisch gesehen ist traditionell an den Einrichtungen der Universitäten und der Universitäten der Künste das Potential an wissenschaftlichen Fachkräften in Forschung und experimenteller Entwicklung im Lande besonders stark konzentriert. Der Umfang der tatsächlich eingesetzten personellen Forschungskapazitäten ist aus den forschungsstatistischen Erhebungen von Statistik Austria ablesbar, die in mehrjährigen Abständen durchgeführt werden.

Die Erhebung über das Jahr 1998 hat an den Universitäten und den Universitäten der Künste insgesamt 5.687,0 Vollzeitäquivalente für direkt in Forschung und experimenteller Entwicklung (F\&E) tätiges wissenschaftliches Personal ergeben. Als gesamte personelle Forschungskapazität inklusive des nichtwissenschaftlichen Personals an Universitäten wurden für 1998 insgesamt 6.814,1 Vollzeitäquivalente (1993: 5.605,2) ermittelt; dies bedeutet einen Zuwachs der universitären Forschungskapazität gegenüber der Erhebung 1993 um 21,6 Prozent. An den Universitäten der Künste waren 1998 insgesamt 66,9 Vollzeitäquivalente in F\&E eingesetzt (1993: 57,4 , das sind $+16,6$ Prozent gegenüber 1993).

Nach Qualifikationen und Tätigkeiten gegliedert, war an den Instituten und Kliniken der Universitäten das wissenschaftliche Personal - das sind Professoren, Universitätsdozenten (sie wurden im Rahmen der F\&E-Erhebung 1998 aus der Personalkategorie Assistenten herausgelöst und erstmals gesondert ausgewiesen!), Assistenten, Wissenschaftliche Hilfskräfte unter Einschluss von Studienassistenten und Demonstratoren, Beamte des wissenschaftlichen Dienstes, sowie gleichwertige Kräfte mit insgesamt 5.623,5 (1993: 4.555,9) Vollzeitäquivalenten vertreten. Für das höherqualifizierte nichtwissenschaftliche Personal (Maturanten, Techniker, Laboranten) ergab die Erhebung 1998 1.190,6 (1993: 1.049,3) Vollzeitäquivalente, für sonstiges Hilfspersonal 1.426,9 (1993: 1.132,7) Vollzeitäquivalente. Die in F\&E eingesetzten Personalkapazitäten haben sich gegenüber 1993 beim wissenschaftlichen Personal somit um 23,4 Prozent erhöht, beim nichtwissenschaftlichen um 13,5 Prozent.

Vergleicht man die Arbeitszeitverteilung der Professoren im Gesamtdurchschnitt (siehe Tabelle 17), so ist festzustellen, dass im Jahre 1998 für F\&E generell mehr Zeit aufgewendet wurde (44,8 Prozent) als für Lehre und Ausbildung (42,1 Prozent).

Betrachtet man jedoch die Arbeitszeitverteilung der Professoren in den einzelnen Wissenschaftszweigen, zeigt sich eine deutliche Differenzierung: Insbesondere in der Humanmedizin und in den Naturwissenschaften wird wesentlich mehr Zeit für F\&E als für Lehre und Ausbildung aufgewendet, wogegen in den Agrarwissenschaften und der Veterinärmedizin sowie in den Geisteswissenschaften der Anteil der der Lehre und Ausbildung gewidmeten Arbeitszeit etwas größer als der auf F\&E entfallende 
Anteil ist. Bei den Technischen Wissenschaften und den Sozialwissenschaften ist der Anteil der für F\&E und der für Lehre und Ausbildung aufgewendeten Arbeitszeit etwa gleich groß.

Bei der Arbeitszeitverteilung der Assistenten ist auch im Jahre 1998 ein besonders deutliches Übergewicht der Forschungstätigkeit (40,9 Prozent ihrer Arbeitszeit) gegenüber der Lehrtätigkeit $(30,4$ Prozent ihrer Arbeitszeit) festzustellen. Die sonstigen Tätigkeiten beanspruchen bei Professoren im Gesamtdurchschnitt rund 13 Prozent ihrer Arbeitszeit, bei Assistenten knapp 29 Prozent, wobei an Kliniken diese Werte (vor allem wegen des Dienstes am Kranken) mehr als doppelt so hoch liegen.

\subsubsection{Zusammenfassung des Personaleinsatzes 1998 für F\&E nach Durchführungs- sektoren}

\section{Hochschulsektor (Tabelle 21 im Anhang)}

Gegenüber 1993 hat der gesamte Personaleinsatz für F\&E (gemessen in Vollzeitäquivalent) um 21,5 Prozent zugenommen, wobei die größte Zunahme bei den auf dem Gebiete der Land- und Forstwirtschaft und Veterinärmedizin tätigen Instituten (+39,0 Prozent), gefolgt von den Kliniken $(+33,2$ Prozent), den auf dem Gebiete der Technischen Wissenschaften (+26,0 Prozent) sowie den auf dem Gebiete der Naturwissenschaften (+25,3 Prozent) tätigen Instituten zu verzeichnen war.

\section{Sektor Staat (Tabellen 22 und 23 im Anhang)}

Gegenüber 1993 hat in diesem Durchführungssektor der Personaleinsatz für F\&E (gemessen in Vollzeitäquivalent) um 0,1 Prozent abgenommen, wobei besonders hohe Rückgänge im land- und forstwirtschaftlichen Bereich (-33,2 Prozent), im humanmedizinischen Bereich (-11,7 Prozent) sowie im Bereich der technischen Wissenschaften (-7,4 Prozent) zu verzeichnen waren. Dagegen hatten die geisteswissenschaftlichen mit $+34,2$ Prozent und die sozialwissenschaftlichen F\&E-Einrichtungen mit +15,0 Prozent deutliche Zuwächse aufzuweisen.

Vergleicht man den Personaleinsatz der F\&E-Einrichtungen für Forschung nach Rechtsträgern in diesem Bereich (Tabelle 24) zeigt sich, dass bei den diesem Durchführungssektor zugeordneten Einrichtungen nur beim Personaleinsatz der im Sektor Staat dominierenden F\&E-Einrichtungen des Bundes empfindliche Rückgänge zu registrieren waren (-14,0 Prozent). Dagegen gab es deutliche Steigerungen des Personaleinsatzes für F\&E bei den Einrichtungen der Kammern (+45,2 Prozent), der Gemeinden (+44,9 Prozent), der überwiegend vom öffentlichen Sektor finanzierten und/oder kontrollierten privaten gemeinnützigen Institutionen (+31,5 Prozent) und der Länder (+3,6 Prozent).

\section{Privater gemeinnütziger Sektor (Tabelle 24 im Anhang)}

Im Rahmen der F\&E-Erhebung 1993 hatten in diesem seit jeher kleinsten nationalen Durchführungssektor aufgrund der Ausgliederung der F\&E-Einrichtungen der Ludwig Boltzmann-Gesellschaft und der "öffentlichen" privaten gemeinnützigen Einrichtungen aus diesem Sektor ${ }^{8}$ sowohl der Personaleinsatz für F\&E als auch die F\&E-Ausgaben empfindliche Rückgänge zu verzeichnen. Zwischen 1993 und 1998 sind in diesem Sektor jedoch die Vollzeitäquivalente für F\&E wieder um insgesamt 47,8 Prozent angewachsen, wobei der Personaleinsatz im Rahmen der einzelnen Wissenschaftszweige eine sehr differenzierte Entwicklung zeigt: Sehr hohe Zuwächse konnten beim Personaleinsatz der naturwissenschaftlichen (+163 Prozent) und der sozialwissenschaftlichen F\&E-Einrichtungen $(+134,3$

\footnotetext{
${ }^{8}$ Revision des "Frascati-Handbuchs" der OECD
} 
Prozent) verzeichnet werden, dagegen ist der Personaleinsatz im Rahmen der geisteswissenschaftlichen Forschung um 71,9 Prozent und der der Technischen Wissenschaften um 26,1 Prozent zurückgegangen.

\section{Unternehmenssektor (Tabellen 25 und 27 im Anhang)}

Gegenüber 1993 hat in den von Statistik Austria erfassten Bereichen unter Ausklammerung des firmeneigenen Bereichs der Personaleinsatz für F\&E (gemessen in Vollzeitäquivalent) insgesamt um 37 Prozent zugenommen, wobei zu vermerken ist, dass der kooperative Bereich eine Steigerung um 41,3 Prozent zu verzeichnen hatte.

Die Daten über den Personaleinsatz des firmeneigenen Bereichs der Jahre 1993 und 1998 sind nicht voll vergleichbar, da im Rahmen der F\&E-Erhebung 1998 die F\&E-Einrichtungen des Dienstleistungssektors erstmals erfasst wurden. Die Steigerung des Personaleinsatzes von 34,7 Prozent seit 1993 im firmeneigenen Bereich ist daher unter Berücksichtigung dieser Tatsache zu beurteilen.

Die Daten der Erhebung 1998 im firmeneigenen Bereich stammen aus der F\&E-Erhebung 1998 der Statistik Austria im firmeneigenen Bereich und wurden der Empfehlung des Frascati-Manuals entsprechend nach der Systematik der Wirtschaftsklassen ISIC, Rev. 3, welche praktisch mit der NACE, Rev. 1, bzw. der ÖNACE identisch ist, aggregiert.

Tabelle 27 im Anhang zeigt die Verteilung des Personaleinsatzes und der Ausgaben für F\&E nach den Wirtschaftsgruppen, denen die F\&E-Aktivitäten dienen. Dabei zeigt sich, dass 1998 (wie auch 1993) der größte Anteil der F\&E-Ressourcen auf die Sachgütererzeugung entfiel (Beschäftigte 83,2 und Ausgaben 83,6 Prozent) ${ }^{9}$.

\footnotetext{
${ }^{9}$ Für detaillierte Ergebnisse vgl. „Forschung und experimentelle Entwicklung (F\&E) im firmeneigenen Bereich 1998“. In: Statistische Nachrichten, Heft 2/2001, S. 89 - 103.
} 


\section{Forschung und Entwicklung im internationalen Vergleich}

\subsection{Forschungsquoten}

Abbildung 3-1: Entwicklung der Forschungsquoten in ausgewählten Ländern, 1993, 1995, 1997, 1999

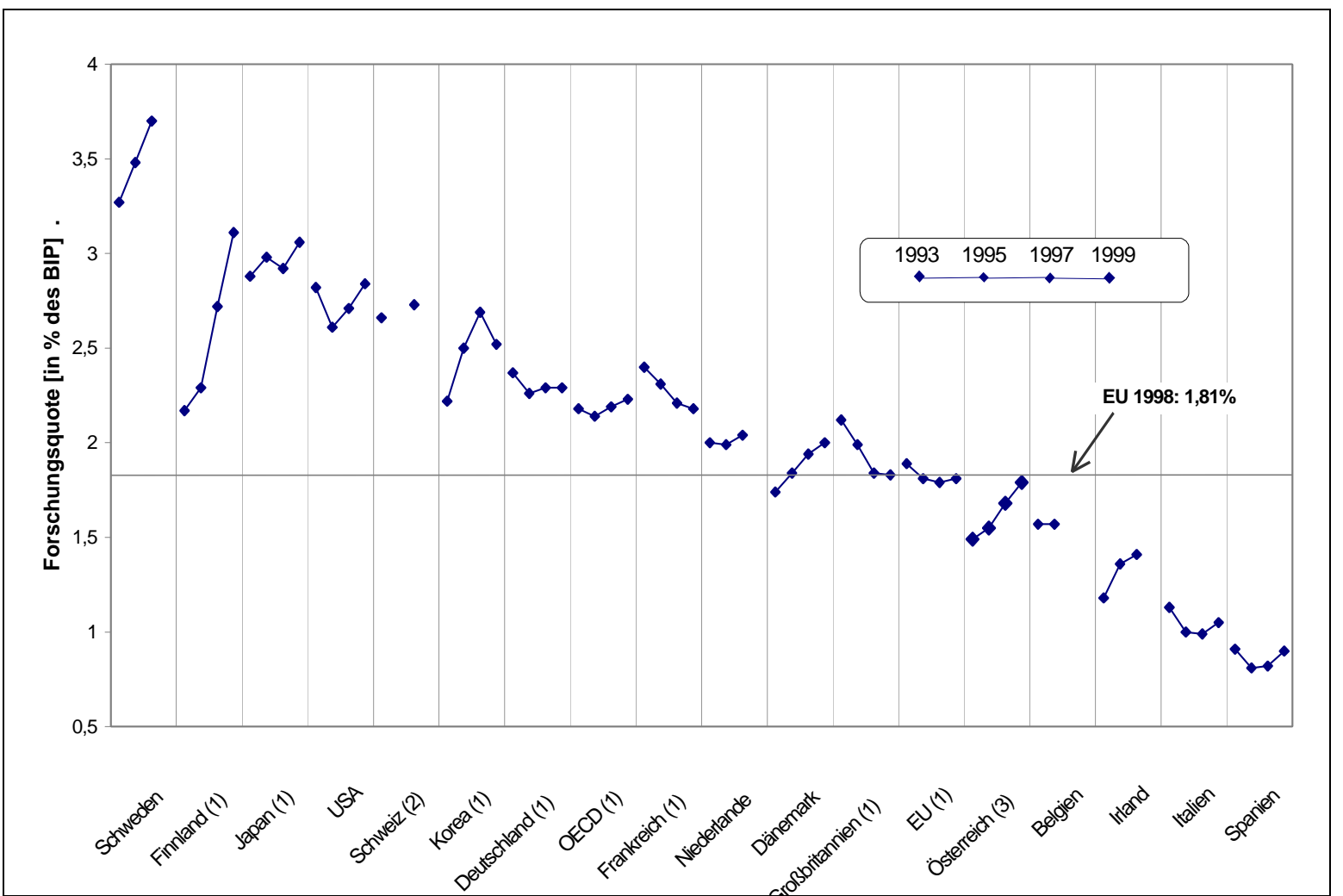

Quelle: OECD-MSTI (2000), Statistik Austria (2000a); (1) letztverfügbarer Wert 1998, (2) nur 1992 und 1996 verfügbar, (3) letztverfügbarer Wert 2000.

Die Aufwendungen für Forschung und Entwicklung (F\&E) sind einer der zentralen Indikatoren für die Bewertung der technologischen Leistungsfähigkeit einer Volkswirtschaft. Über die Forschungsquote, d.h. den Anteil der F\&E-Aufwendungen gemessen am Bruttoinlandsprodukt (BIP), werden einfache internationale Vergleiche zwischen den F\&E-Inputs der verschiedenen Länder angestellt. Abbildung 3-1 zeigt die Entwicklung in Österreich und einigen Vergleichsländern seit 1993. Eine Übersicht über den OECD-Bereich - bezogen auf das Jahr 1998 bietet Tabelle 27 im statistischen Anhang.

Sowohl in der EU als auch in der OECD weist der internationale Trend über die letzten Jahre eine Stagnation der F\&E-Quoten auf. Lediglich einige kleine Länder wie Schweden, Finnland, Dänemark und Irland haben ihre F\&E-Quoten beträchtlich erhöht und konnten damit z.T. ins internationale Spitzenfeld vordringen. Besondere Beachtung verdient der technologische Aufholprozess Finnlands, das Anfang der 80er Jahre vom gleichen Niveau wie Österreich startete und 1998 - trotz einer schweren Wirtschaftskrise zu Beginn der 90er Jahre - eine F\&E-Quote von 2,89 \% erreichte.

In Österreich wurden die Forschungsquoten der letzten Jahre durch Statistik Austria im Rahmen der Globalschätzung 2000 - nach einer Revision der F\&E-Statistik aus dem Jahr 1999 - leicht nach oben korrigiert. Demnach konnte Österreich in seinem Aufholprozess zum EU-Durchschnitt aufschließen (1998 Österreich: 1,81 \%; EU: 1,81\%). Dem Durchschnitt der OECD-Staaten (1998: 2,18 \%) gegenüber weist Österreich dagegen weiterhin einen deutlichen Rückstand auf, der insbesondere aus der Gewichtung der USA und Japans innerhalb der OECD resultiert. Die österreichische Bundesregierung 
hat in der Erklärung vom 11. Juli 2000 ihr Ziel bekräftigt, eine Erhöhung der F\&E-Quote auf 2,5\% des BIP bis zum Jahr 2005 zu erreichen.

\subsection{Finanzierung von Forschung und Entwicklung im internationalen Vergleich}

Abbildung 3-2: Finanzierung der Forschung nach Sektoren im internationalen Vergleich, 1998

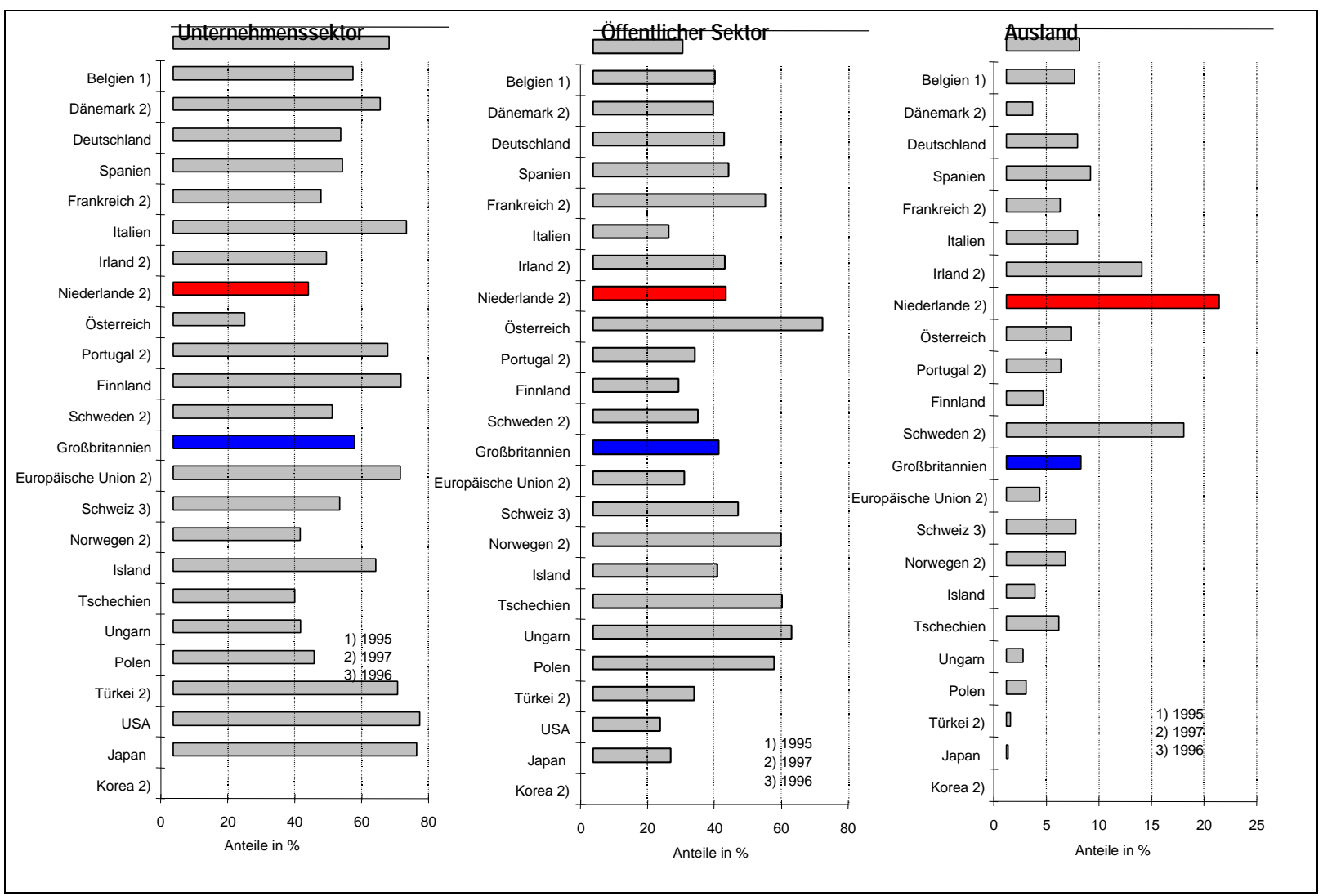

Quelle: OECD- MSTI, Statistik Austria.

Der Anteil der heimischen Unternehmen an der Finanzierung von F\&E liegt deutlich unter dem EUDurchschnitt und befindet sich in etwa auf dem Niveau von Italien, den Niederlanden, Ungarn oder Polen. In Ländern mit dynamischer Entwicklung bzw. hohem Niveau tragen heimische Unternehmen deutlich mehr zum gesamten Finanzvolumen bei.

In Österreich liegt der Finanzierungsanteil des öffentlichen Sektors an den F\&E - Ausgaben in etwa im EU-Durchschnitt. Hochentwickelte und technologisch dynamische Länder weisen generell einen niedrigeren Finanzierungsanteil der öffentlichen Hand und gleichzeitig einen höheren Finanzierungsanteil des Unternehmenssektors auf. Die vom öffentlichen Sektor finanzierten F\&E-Ausgaben werden vor allem an den Universitäten durchgeführt. Ein Teil geht jedoch auch als Forschungsförderungen an den Unternehmenssektor (vergleiche Abbildung 3-2).

Der auslandsfinanzierte Teil der F\&E-Ausgaben umfasst die Forschungsaktivitäten von Töchtern multinationationaler Konzerne in Österreich, die durch die Muttergesellschaft finanziert werden, Forschungsaufträge aus dem Ausland an österreichische Institutionen und Unternehmen, sowie Forschungsgelder, die über die Beteiligung an den FTE-Programmen der EU nach Österreich geflossen sind. 
Nach der Revision der amtlichen F\&E-Statistik hat Österreich im OECD-Raum den bei weitem höchsten Anteil auslandsfinanzierter F\&E. Da sich die Erfassung der F\&E-Finanzierungsquellen seit der letzten F\&E-Erhebung 1993 geändert hat, gibt es keinen direkten Vergleichswert, der eine eindeutige Interpretation dieses Wertes erlaubt. Laut Statistik Austria handelt es sich primär um die Finanzierung von im Inland durchgeführter F\&E-Aktivitäten durch ausländische Unternehmen. Die Rückflüsse aus FTE-Programmen der EU sind in der zweiten Hälfte der neunziger Jahre zwar angestiegen, tragen zur absoluten Höhe dieses Wertes jedoch nur einen kleinen Teil bei.

Wenn man davon ausgeht, dass der überwiegende Teil der durch das Ausland finanzierten F\&EAusgaben durch den Unternehmenssektor durchgeführt wird, dann sollte der Durchführungsanteil des Unternehmenssektors seit der F\&E-Erhebung 1993 deutlich gewachsen sein. Die Aufschlüsselung der F\&E-Ausgaben nach Durchführungssektoren, basierend auf der F\&E-Erhebung 1998, lag zur Zeit der Ausarbeitung des Berichts noch nicht vor; sie ist jedoch sowohl für die Interpretation der Finanzierungswerte als auch für die technologiepolitische Strategiefindung unerlässlich. 


\subsection{Durchführung von Forschung und Entwicklung im internationalen Vergleich}

Abbildung 3-3 : F\&E-Ausgaben nach durchführenden Sektoren

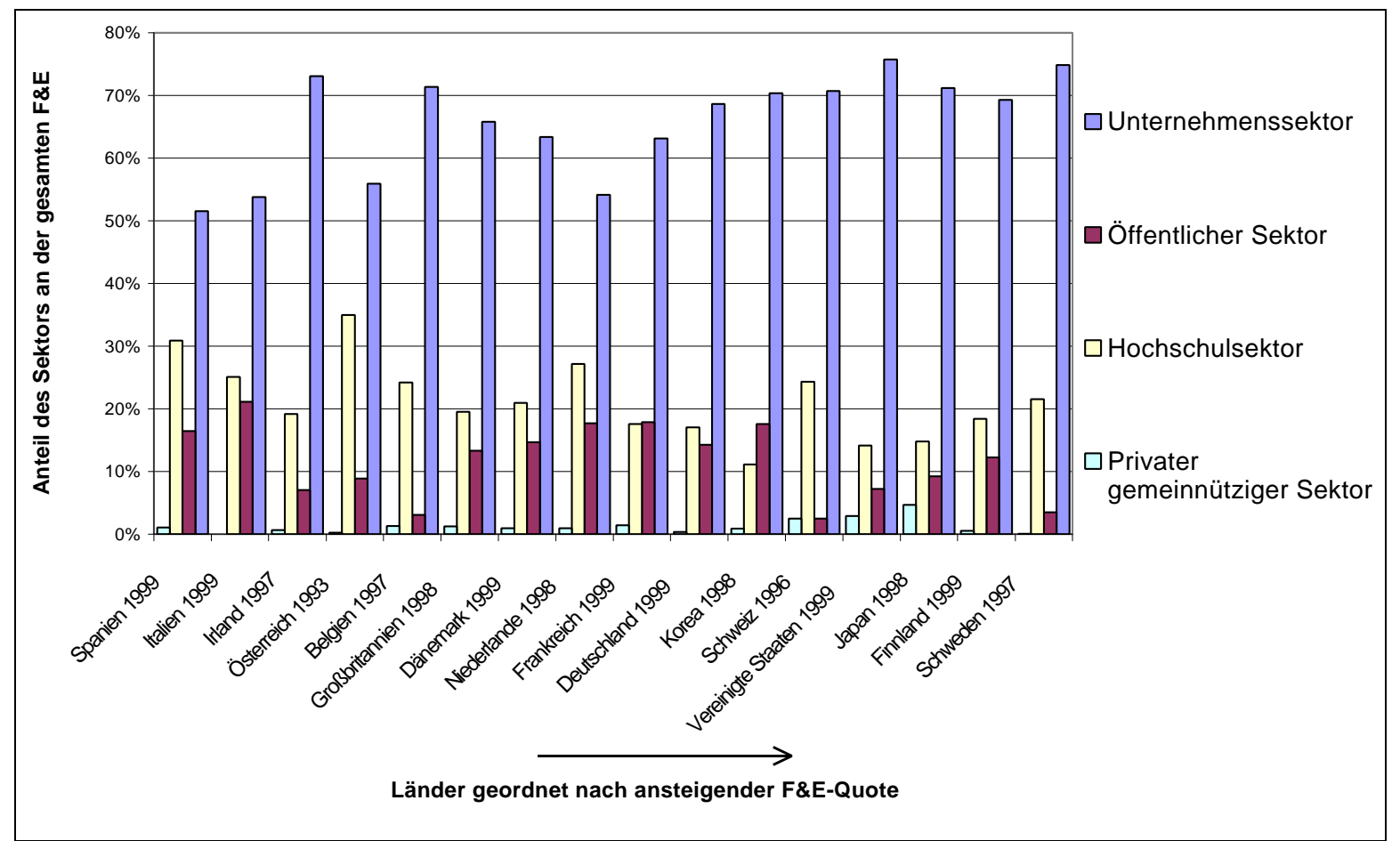

Quelle: OECD-BSTS (2000).

Im internationalen Vergleich ist zu beobachten, dass Länder mit hoher F\&E-Quote diese Position vor allem einem deutlich überproportionalen F\&E- Anteil des Unternehmenssektors zu verdanken haben. Die Höhe der gesamtwirtschaftlichen Forschungsquote korreliert daher mit dem Ausmaß der F\&EAktivitäten der Unternehmen. Demgegenüber sind die Anteile des öffentlichen Sektors und des Hochschulsektors bei höherer F\&E-Quote im allgemeinen geringer. Die Forschungsquote eines Landes wird daher nicht zuletzt durch die sektorale Organisation seiner Forschungsaktivitäten bestimmt.

Die höchsten Anteile des Unternehmenssektors an den gesamten Forschungsaufwendungen weisen mit $76 \%$ die Vereinigten Staaten (1999), Schweden mit $75 \%$ (1997), sowie Irland mit 73 \% (1997) auf. Im EU-Schnitt beträgt der Unternehmensanteil etwa $62 \%$, in der OECD über $68 \%$. In Österreich hingegen liegt der Anteil der Unternehmen an der durchgeführten F\&E trotz jüngster Indizien für einen Aufholprozess noch immer deutlich unter dem Durchschnitt. Die letzte Erhebung zur F\&E in Österreich (Basisjahr 1993), für die vollständig publizierte Ergebnisse vorliegen, weist einen Unternehmensanteil von $56 \%$ aus. Entsprechend hoch ist der Anteil der Hochschulen, mit international unübertroffenen $35 \%$ der gesamten österreichischen F\&E-Ausgaben ${ }^{10}$.

\footnotetext{
${ }^{10}$ In Österreich sind die durchführenden Sektoren wie folgt abgegrenzt: Unternehmenssektor (firmeneigener Bereich einschliesslich Kraftwerksgesellschaften, und kooperativer Bereich einschließlich Österreichisches Forschungszentrum Seibersdorf, Joanneum Research; sowie die Ziviltechniker); Sektor Staat (Bundesinstitutionen (unter Ausklammerung der im Hochschulsektor zusammengefassten), Landes-, Gemeinde-, Kammerinstitutionen sowie Einrichtungen von Sozialversicherungsträgern, Museen und Landeskrankenanstalten); Hochschulsektor (Universitätsinstitute und -kliniken, Universitäten der Künste, Österreichische Akademie der Wissenschaften sowie Versuchsanstalten an HTLs), sowie privater gemeinnütziger Sektor, der private Institutionen ohne Erwerbscharakter, mit vorwiegend privatem oder privatrechtlichem, konfessionellem oder sonstigem nicht öffentlichem Status umfasst, sofern sie nicht zur Gänze oder überwiegend aus öffentlichen Mitteln dotiert werden.
} 


\subsection{Forschung und Entwicklung im Unternehmenssektor (internationaler Vergleich)}

Abbildung 3-4: Unternehmensforschung (BERD) nach Branchengruppen im internationalen Vergleich

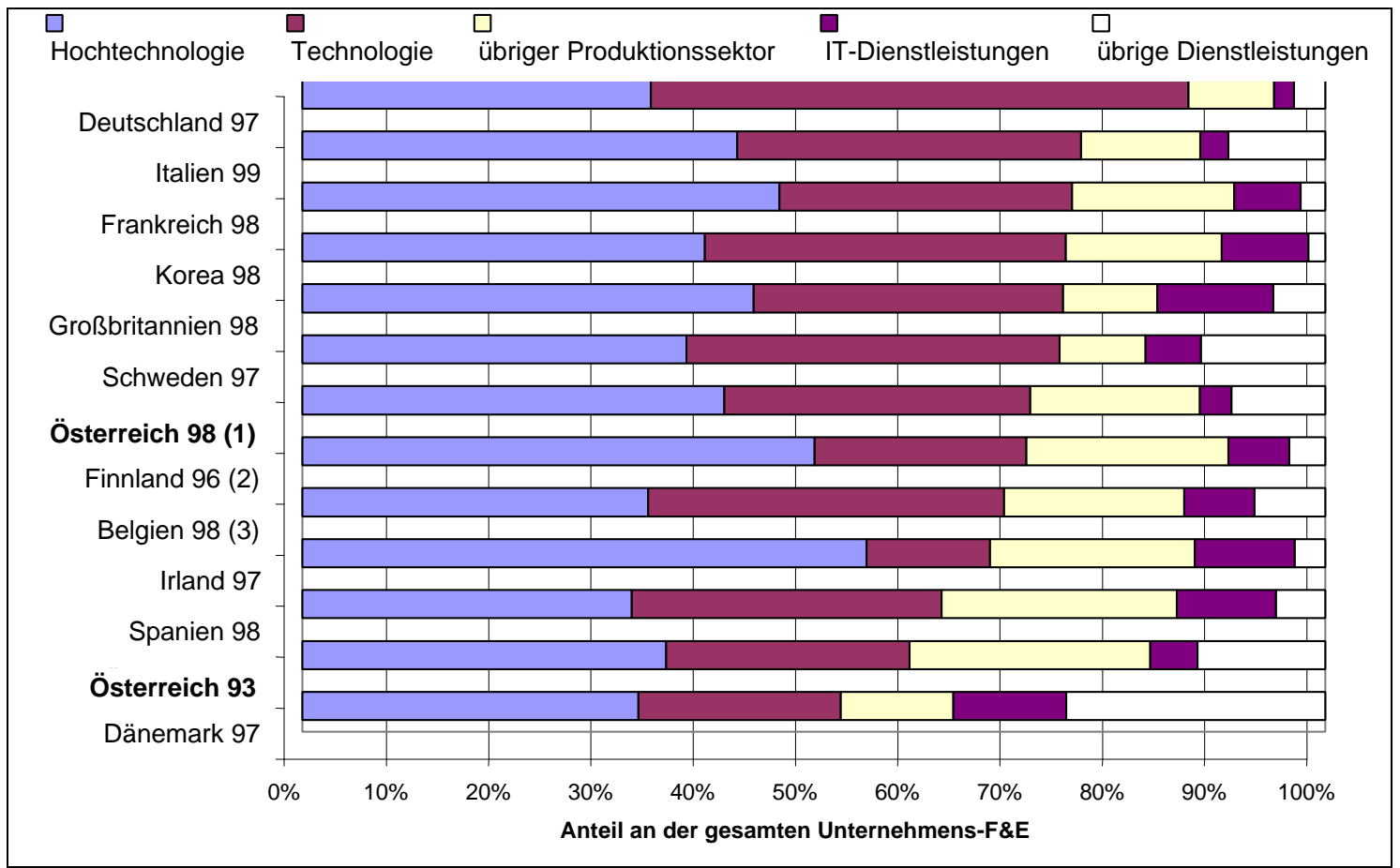

Quelle: OECD-BSTS (2000), Statistik Austria (2001), Berechnungen von tip. Abgrenzung der Branchengruppen nach Klassifikation ISIC rev. $3^{11}$. (1) $92 \%$ der gesamten Unternehmens-F\&E inkludiert. (2) $83 \%$ der gesamten Unternehmens-F\&E inkludiert. (3) $95 \%$ der gesamten Unternehmens-

F\&E inkludiert.

Ein internationaler Strukturvergleich der Forschungsausgaben des Unternehmenssektors ist aufgrund der abweichenden Datenerhebung in einzelnen Ländern schwierig. Fasst man jedoch die einzelnen Branchen zu fünf Gruppen, nämlich Hochtechnologie, Technologie, sonstige Produktionssektoren, Dienstleistungen und Informationstechnologie (IT)-bezogene Dienstleistungen zusammen, so lässt sich grob folgendes Bild zeichnen (vergleiche Abbildung 3-4). Die Unternehmensforschung wird in allen Vergleichsländern zum überwiegenden Anteil in den Sektoren Technologie und Hochtechnologie durchgeführt. Im Durchschnitt der betrachteten Länder liegt ihr gemeinsamer Anteil bei $71 \%$, wobei der mehrheitliche Anteil zumeist bei der Hochtechnologie liegt.

Einige Länder, die während der letzten Jahre ihre F\&E-Quoten besonders rasch erhöhen konnten, zeichnen sich durch eine Dominanz der Hochtechnologie in der Unternehmensforschung aus. In Irland etwa beträgt ihr Anteil an den gesamten Forschungsausgaben des Unternehmenssektors $55 \%$, in Finnland über $50 \%$, wobei die besonders forschungsintensive pharmazeutische Industrie in Finnland nicht getrennt erhoben wird, und daher im vorliegenden Vergleich gar nicht zum Tragen kommt. Einzig in Deutschland liegt der Schwerpunkt der Aufwendungen mit $53 \%$ auf der (mittleren) Technologie;

\footnotetext{
${ }^{11}$ Für die vorliegende Darstellung wurden die Sektoren nach dem International Standard of Industrial Classification (ISIC) rev. 3 in folgender Weise gruppiert:

Hochtechnologie: 19 (Pharmazeutika), 28 (Büromaschinen, Computer), 30 (Elektronische Geräte), 33 (Instrumente), 37 (Flugzeugbau); Technologie: 16 (Mineralölverarbeitung), 18 (Chemikalien ohne Pharmazeutika), 27 (Maschinenbau), 29 (Elektrogeräte), 34 (Fahrzeugbau), 36 (Schiffe), 38 (Sonstiger Fahrzeugbau); Sonstige Produktionssektoren: ISIC rev. 3Sektoren 1 bis 44 ohne Hochtechnologie und Technologie; IT-Dienstleistungen: 48 (Transport), 49 (Kommunikation), 54 (Computerbezogene Dienstleistungen); Sonstige Dienstleistungen: 46 (Handel), 47 (Hotellerie), 52 (Finanzdienstleistungen), 57 (Forschung und Entwicklung), 58 (Andere Unternehmensaktivitäten), 59 (Persönliche Dienstleistungen etc.).
} 
dagegen liegt Deutschland bei der Hochtechnologie mit einem Anteil von $35 \%$ im Schlussfeld der Vergleichsländer.

In Österreich konnten nach neuen Zahlen von Statistik Austria (2001) ${ }^{12}$ gegenüber 1993 die Unternehmen der Technologie und Hochtechnologie ihren gemeinsamen Anteil an der gesamten Unternehmens-F\&E deutlich erhöhen, und erreichten im Jahr 1998 einen Anteil von 71 \%, was dem Durchschnitt der betrachteten Länder entspricht. Dies trotz der Tatsache, dass die Branchengruppe Technologie im vorliegenden Vergleich nicht vollständig erfasst ist, und in Wirklichkeit etwas besser positioniert sein dürfte ${ }^{13}$. Es bleibt jedoch auf den weiterhin beträchtlichen Rückstand Österreichs auf die Spitzenreiter wie Finnland oder Irland hinzuweisen, was den Anteil der Hochtechnologie an der gesamten Unternehmens-F\&E (41\%) betrifft.

\subsection{Grundlagenforschung im internationalen Vergleich}

\section{Abbildung 3-5: Anteil der Grundlagenforschung nach ausführenden Sektoren und Ländern 1997} $\square$ (in \% des BIP sovie in \% der gesänten F\&E Ausgaben)

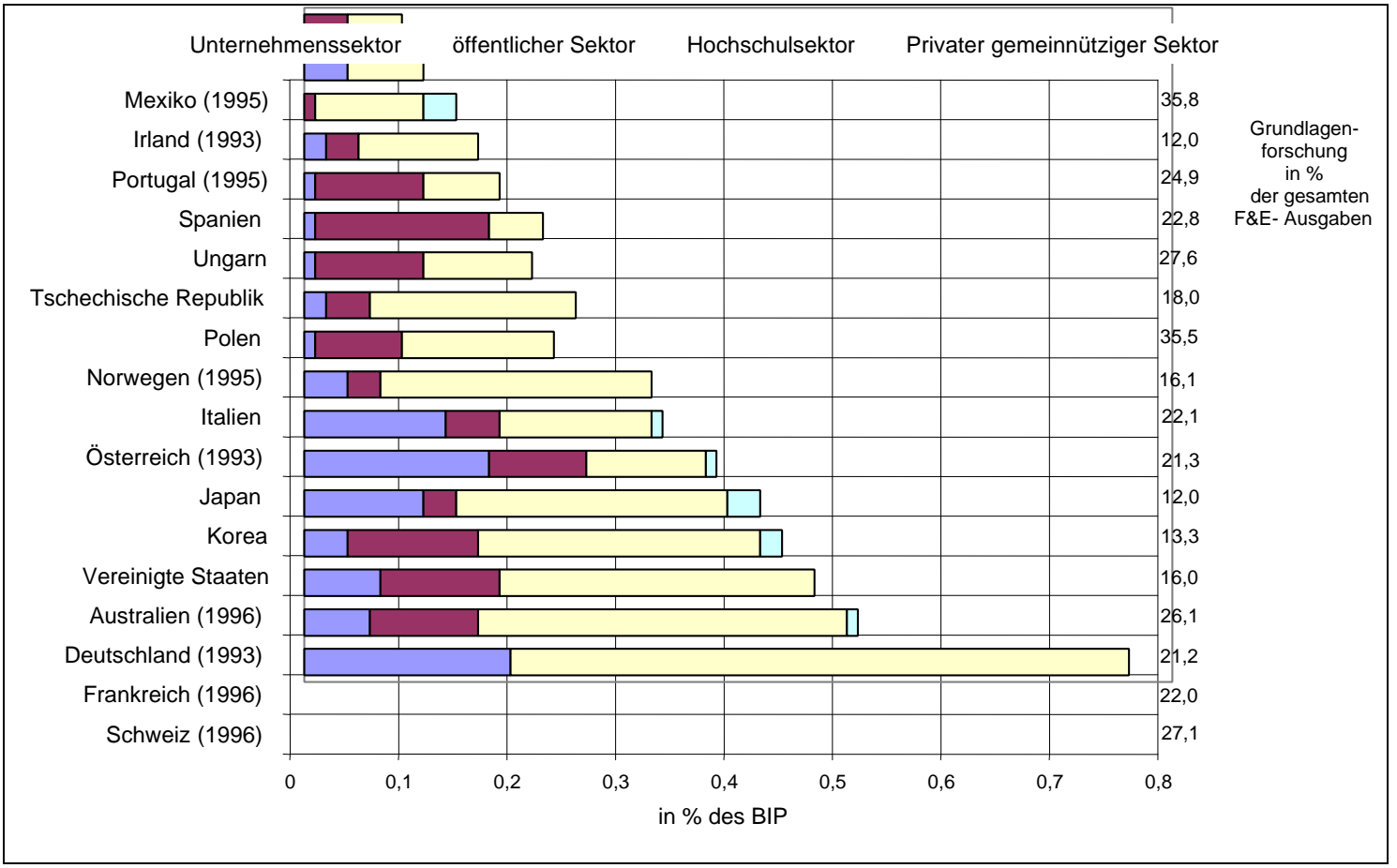

Quelle: OECD (1999)

\section{Grundlagenforschung nach ausführenden Sektoren}

Innerhalb der OECD Länder variieren die Ausgaben für Grundlagenforschung zwischen etwa 0,1\% (Mexiko) und mehr als 0,7\% (Schweiz) des BIP. Österreich liegt in diesem Vergleich mit 0,32\% des BIP im Mittelfeld. Verglichen mit den frühen 80er Jahren stieg der Anteil der Grundlagenforschung gemessen am BIP in den meisten OECD Ländern deutlich an.

Ebenfalls weit gestreut sind die Anteile der Ausgaben für Grundlagenforschung gemessen an den gesamten F\&E Ausgaben. Während Länder wie Mexiko und Polen Werte über $30 \%$ aufweisen, liegt der Anteil der Grundlagenforschung an den gesamten F\&E Aufwendungen in ostasiatischen Ländern

\footnotetext{
${ }^{12}$ Für Österreich liegen Teilergebnisse der ersten F\&E-Erhebung der Statistik Austria betreffend das Jahr 1998 vor. Für den Unternehmenssektor (mit Ausnahme des kooperativen Bereichs und der Ziviltechniker) sind damit erstmals Ergebnisse auch für den Dienstleistungssektor verfügbar.

${ }^{13}$ Aus Gründen geringer Unternehmensanzahl in einzelnen Branchen besteht für die Branchensumme Datenschutz.
} 
wie Japan und Korea bei 12-13\%. Österreich liegt ähnlich wie Deutschland (21,2\%), Frankreich (22\%) und Italien (22,1\%) mit 21,2\% (1993) im Mittelfeld.

Innerhalb der OECD wird der größte Teil der Grundlagenforschung im Hochschulsektor und/oder im öffentlichen Bereich durchgeführt. Für die meisten EU Staaten ist dieses Bild charakteristisch, während in der Schweiz, USA, Korea und Japan die Grundlagenforschung im Unternehmenssektor eine deutlich größere Rolle spielt. Auch in Österreich dominiert der Hochschulsektor in der Durchführung von Grundlagenforschung, der öffentliche Sektor und der Unternehmenssektor spielen eine vergleichsweise geringe Rolle.

\section{Ausgaben der Hochschulforschung im internationalen Vergleich (HERD)}

Tabelle 3-6: Forschungsausgaben im Hochschulsektor (in \% des BIP)

\begin{tabular}{|c|c|c|c|c|c|c|}
\hline & 1989 & 1993 & 1995 & 1997 & 1998 & 1999 \\
\hline Deutschland & 0,41 & 0,43 & 0,41 & 0,41 & 0,4 & 0,41 \\
\hline Frankreich & 0,35 & 0,38 & 0,39 & 0,38 & 0,37 & - \\
\hline Italien & 0,24 & 0,28 & 0,25 & 0,26 & 0,25 & 0,26 \\
\hline Österreich & 0,44 & 0,52 & - & - & - & - \\
\hline
\end{tabular}

Quelle: OECD-MSTI (2000)

Tabelle 3-6 stellt die Entwicklung der Ausgaben für Hochschulforschung (HERD - Higher Education Expenditure on Research and Development) der größten EU-Länder, der EU insgesamt und Österreichs seit 1989 dar. Der internationale Vergleich des HERD-Anteils am BIP widerspiegelt den Stellenwert der Hochschulforschung in dem jeweiligen Land. In Österreich lag dieser 1993 - dem Jahr der letzten Vollerhebung - mit 0,52\% deutlich über dem Wert von 0,37\%, den die OECD 1998 für den Durchschnitt der EU-Staaten angibt. Übertroffen wird Österreichs Hochschulanteil nur von Schweden (1997: 0,8\%).

Insgesamt scheint die Bedeutung der Hochschulforschung leicht zurückgegangen zu sein. Während innerhalb der Europäischen Union in der ersten Hälfte der 90er Jahre (ebenso wie in den 80er Jahren) der HERD-Anteil am BIP deutlich gestiegen ist, lässt sich in der zweiten Hälfte ein leichter Rückgang der HERD-Anteile von 0,39 \% des BIP im Jahr 1993 auf 0,37\% 1998 feststellen. Diese allgemeine Entwicklung spiegelt sich mit Ausnahme von Schweden in allen angeführten Ländern wider. 


\section{Internationalisierung der österreichischen Forschung und Entwicklung}

\subsection{Die österreichische Beteiligung am EU-Rahmenprogramm}

In Österreich wurde im April 2000 die vom BMBWK beauftragte Evaluierung der österreichischen Beteiligung am 4. Rahmenprogramm der EU fertiggestellt (Schibany et al. 2001). Im Rahmen dieser Studie wurde neben einer Einschätzung des Stellenwertes der Rahmenprogramme im europäischen Forschungsraum auch eine Primärerhebung durchgeführt, woraus wichtige Schlussfolgerungen bezüglich der Auswirkung der Rahmenprogramme auf kooperative, vorwettbewerbliche und angewandte Forschung in Österreich gezogen werden konnten.

\subsubsection{Die Präsenz im 4. Rahmenprogramm}

Tab. 4.1: Die Präsenz Österreichs im 4. RP (Aktionslinien 1-4)

\begin{tabular}{lcc}
\hline & In eingereichten Projekten & In erfolgreichen Projekten \\
\hline Beteiligungen & 7.164 & 1.923 \\
Projekte mit österreichischer Beteiligung & 5.680 & 1.444 \\
Koordinatorlnnen & 1.124 & 270 \\
Organisationen & 2.320 & 1.009 \\
Rückflüsse & & 192.627 Mio. EURO (2,65 Mrd. ATS) \\
\hline
\end{tabular}

Quelle: BIT

- Mit der Mitgliedschaft in der EU hat die Beteiligung Österreichs an den Rahmenprogramm einen Quantensprung erfahren: die Zahl der Beteiligungen im 4. Rahmenprogramm vervierfachte sich im Vergleich zu der Beteiligung in den vorhergehenden RP zusammengenommen.

- Im gesamten 4. Rahmenprogramm waren insgesamt 2.320 österreichische Organisationen in eingereichten Vorschlägen beteiligt. Davon waren 1.009 Organisationen in erfolgreichen Anträgen mit insgesamt 1.444 Projekten und 1.923 Beteiligungen. Durchschnittlich entfielen 1,33 österreichische Beteiligungen in Projekten mit österreichischer Präsenz.

- Die gesamten Rückflüsse aus der Beteiligung am 4. Rahmenprogramm betragen über 192 Mill. EURO ( 2,65 Mrd. ATS) und stellen einen Anteil von 1,99 \% der gesamten für indirekte Aktionen vorgesehenen Mittel dar.

- Von den $1.009 \mathrm{im} \mathrm{4.} \mathrm{Rahmenprogramm} \mathrm{vertretenen} \mathrm{österreichischen} \mathrm{Organisationen} \mathrm{entfallen} 50$ $\%$ auf Industrieunternehmen, $28 \%$ auf Universitäten und $13 \%$ auf außeruniversitäre Forschungseinrichtungen. Betrachtet man jedoch die Verteilung in bezug auf erfolgreiche Beteiligungen, zeigen sich deutliche Unterschiede in der durchschnittlichen Zahl an Beteiligungen pro Organisation. Auf Unternehmen kommen durchschnittlich 1,5 Beteiligungen, auf Universitätsinstitute 2,1 und auf außeruniversitäre Forschungsinstitute durchschnittlich 2,3 Beteiligungen. 


\subsubsection{Determinanten der Beteiligung}

\section{Abbildung 4-1: Zusammenhang zwischen F\&E-Personal und Zahl der}

erfolgreichen Beteiligungen

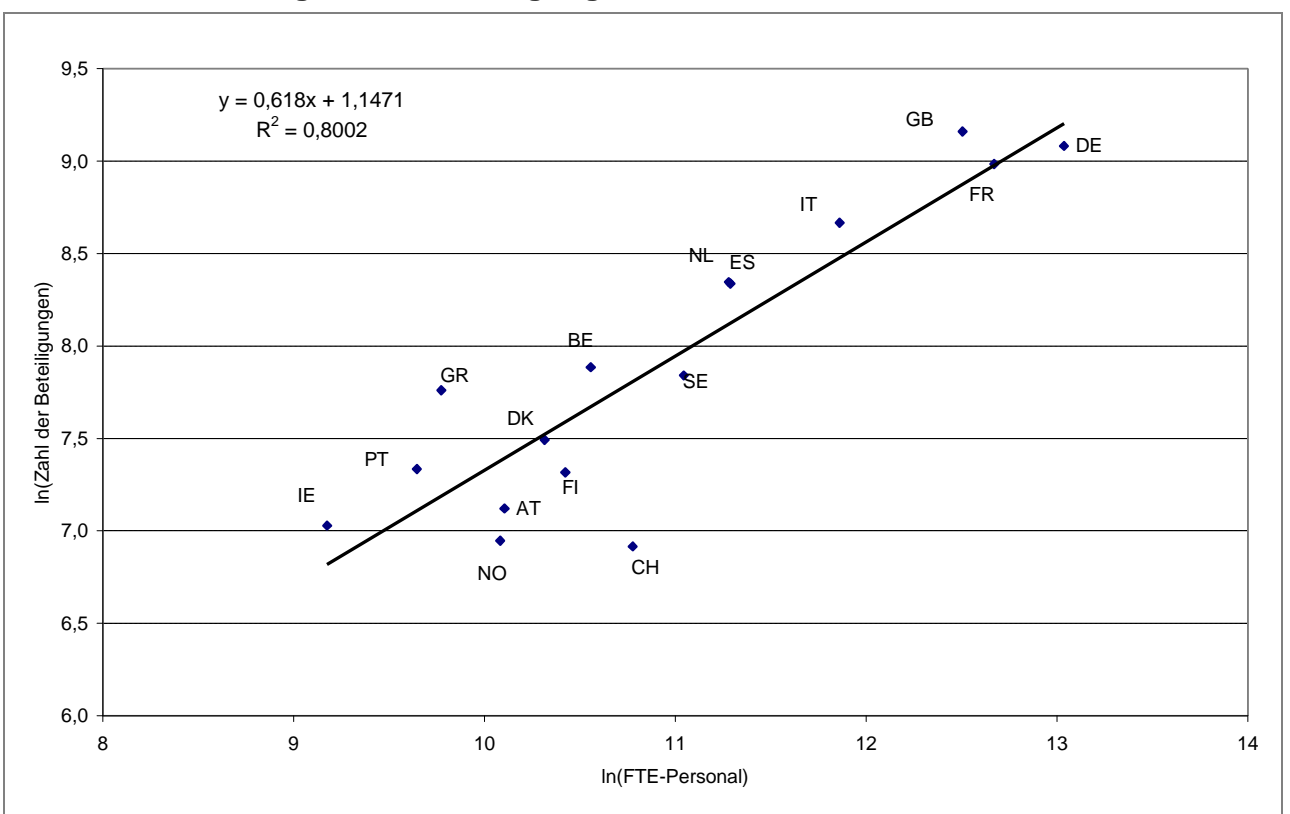

Quelle: Schibany et al. 2001

- $\quad$ Die Beteiligung nach Mitgliedsländern und die Anzahl des FTE-Personals zeigt einen eindrucksvollen und erklärenden Zusammenhang. Das vorhandene Absorptionspotential in Form von Humanressourcen erklärt 80 \% der Varianz der erfolgreichen Beteiligungen eines Landes. Die restlichen $20 \%$ sind somit auf den Einfluss anderer Faktoren zurückzuführen. Länder, die unterhalb der Trendgeraden liegen, haben demgemäß eine zu "geringe“ Anzahl an Beteiligungen, was für die Nicht-EU-Mitgliedstaaten Norwegen und Schweiz am deutlichsten erkennbar ist.

- $\quad$ Trotz des raschen Anstiegs der österreichischen Beteiligung am 4. Rahmenprogramm dürfte noch immer ein unausgeschöpftes Aufholpotential bestehen. Dies trifft auch auf die beiden anderen neuen Mitgliedsstaaten Schweden und Finnland zu, wobei Österreich allerdings deutlich unterhalb der Trendgeraden liegt.

- $\quad$ Eine Analyse der Beteiligungsmuster zeigt weiters, dass das Rahmenprogramm einen (durchaus intendierten) Effekt in Richtung europäischer Konvergenz hat: die Struktur der Beteiligung der einzelnen Länder wird im wesentlichen von der Struktur der Rahmenprogramme bestimmt und weist eine hohe Korrelation zwischen den beteiligten Ländern auf. Unterschiedliche nationale Spezialisierungen schlagen sich nur in geringem Ausmaß nieder. 


\subsubsection{Kosten - Nutzeneinschätzung}

Abbildung 4-2: Kosten-Nutzenvergleich nach Organisationstyp

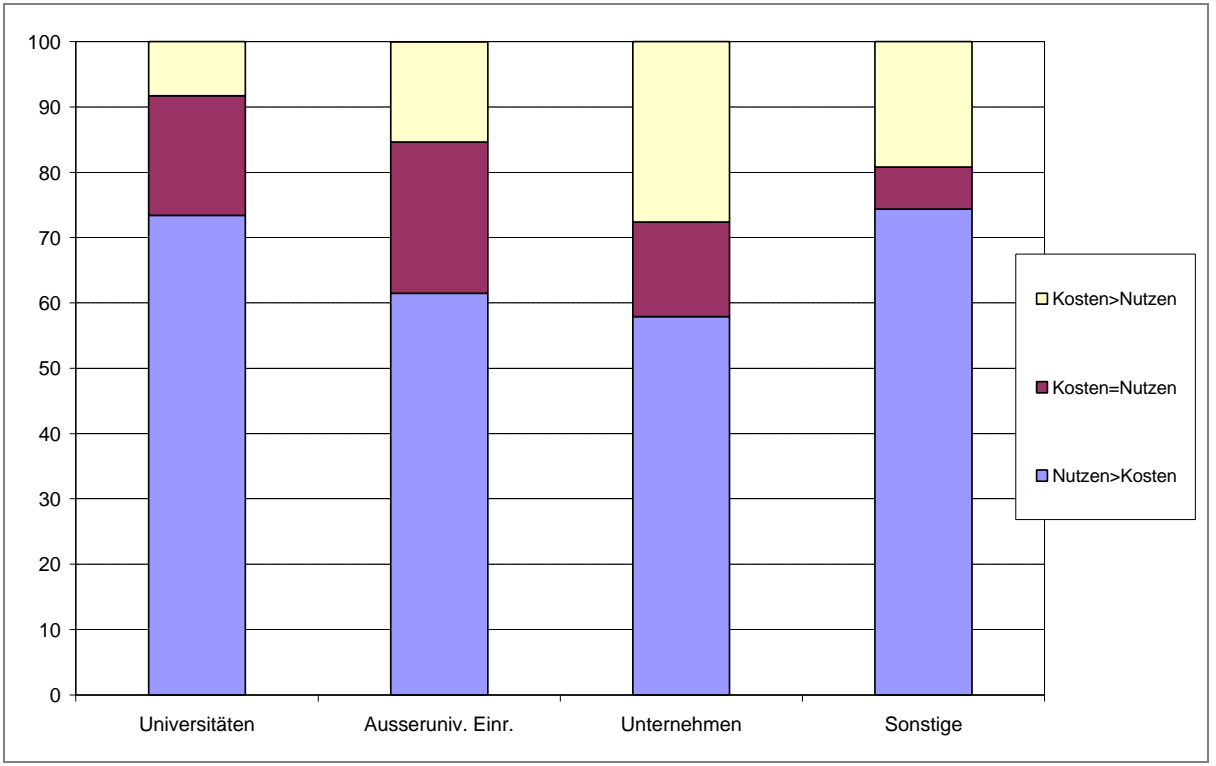

Quelle: Schibany et al. 2001

- Die Frage nach der generellen Einschätzung des Nutzens einer Beteiligung am 4. Rahmenprogramm zeigt ein durchaus positives Bild: $66 \%$ der Teilnehmer schätzen den Nutzen ihrer Teilnahme höher ein als die daraus entstandenen Kosten. $17 \%$ sehen ein ausgeglichenes Verhältnis zwischen Nutzen und Kosten und nur $17 \%$ beurteilen das Verhältnis negativ.

- Universitäten und außeruniversitäre Institute geben ein durchwegs positiveres Bild als die Teilnehmer aus dem Unternehmenssektor, was mit den geringeren Opportunitätskosten zu erklären ist.

- Die generelle Nutzeneinschätzung fällt darüber hinaus auch bei Newcomern positiv aus: über zwei Drittel schätzen das Nutzen/Kosten-Verhältnis positiv ein, womit sie sogar knapp über jener Gruppe liegen, die bereits an früheren Rahmenprogrammen teilgenommen haben.

- Die Forschungsarbeit in EU-Projekten ist vorwettbewerblich ausgerichtet, was Einfluss auf den Stellenwert eines EU-Projektes im Kontext einer Forschungsstrategie hat. Für Unternehmen stellen die Rahmenprogramme die Möglichkeit dar, Forschungsaktivitäten in Kooperation durchzuführen, welche von einer zukünftigen strategischen Bedeutung sein können. Derartiges Wissen wird zunehmend in größeren Konsortien entwickelt und trägt zu Kompetenzaufbau bei. Für den Großteil der teilnehmenden Unternehmen unterstützt die Teilnahme am Rahmenprogramm andere Innovationsaktivitäten. Universitäten und außeruniversitäre Einrichtungen hingegen messen dem EU-Projekt eine wesentlich höhere strategische Bedeutung zu - was in ursächlichem Zusammenhang mit wissenschaftlicher Forschung steht. 


\subsubsection{Kurz- und mittelfristige Wirkungen der Teilnahme}

Abbildung 4-3: Kurz- und mittelfristige Wirkungen der Teilnahme an RP-Projekten
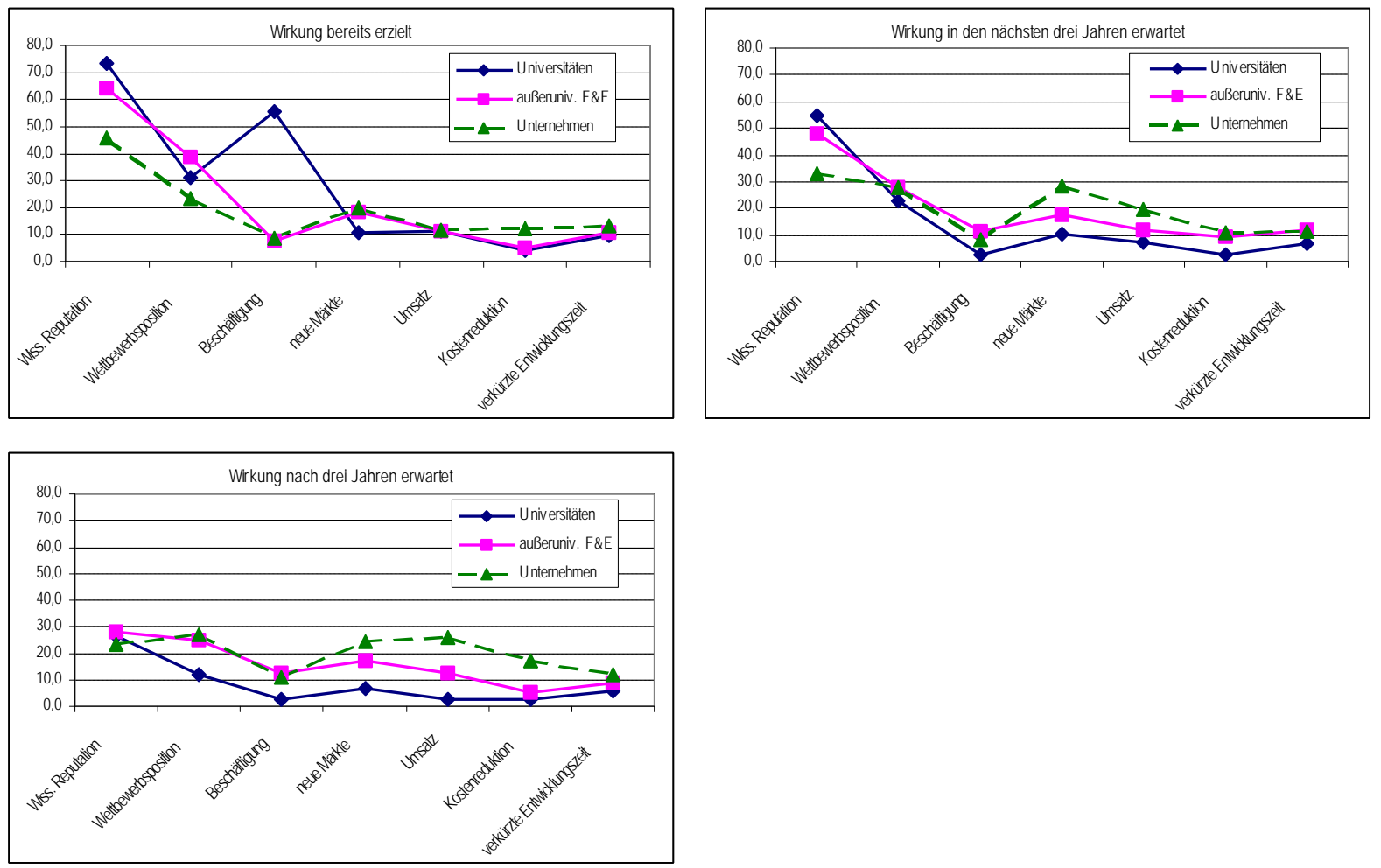

Quelle: Schibany et al. (2001)

- Hinsichtlich der Wirkungen der Teilnahme an den EU- Rahmenprogrammen liegt die technologische und/oder wissenschaftliche Reputation an erster Stelle. Offensichtlich ist für Universitäten (73\% gaben an, dass sie stark positive Wirkungen auf die Reputation wahrnehmen) und außeruniversitäre Forschungszentren die Teilnahme bereits ein wichtiges Instrument, um nach außen ihre wissenschaftliche/technologische Leistungsfähigkeit zu signalisieren.

- Was die kurzfristigen Wirkungen auf das Beschäftigungsniveau betrifft, führen die universitären Forschungseinrichtungen mit einem Anteil von $56 \%$, die angeben, dass bereits starke positive Auswirkungen auf die Beschäftigung ausgehen. Dies ist insofern nicht überraschend, da im Rahmen von universitären Projekten meist direkt Doktoranden bzw. post-docs eingesetzt werden, die projektbezogen zusätzlich zum Stammpersonal beschäftigt werden. Der Anreiz, gerade für die Durchführung von EU-Projekten verstärkt den akademischen Nachwuchs einzubinden, ist nicht zuletzt darauf zurückzuführen, dass sich die EU-Förderung bei Universitäten auf die Grenzkosten des Projektes beziehen, also Stammpersonal nicht finanziert wird. Die Ergebnisse zeigen, dass auf universitärer Ebene die Teilnahme an EU-Rahmenprogrammen für den akademischen Nachwuchs bereits eine wichtige Rolle spielt. In den außeruniversitären Einrichtungen bzw. den Unternehmen werden die Projekte hingegen vom Stammpersonal durchgeführt, sodass hier die direkten Wirkungen während bzw. kurz nach Projektabwicklung entsprechend weniger oft genannt wurden.

- Die mittelfristigen Wirkungen zeigen, dass die Teilnahme an den EU-Programmen auch als offensives mittelfristiges Instrument zur Markterschließung eingesetzt wird. Obwohl die Rahmenprogramme grundsätzlich vorwettbewerblich organisiert sind, zeigen sich (teilweise sogar kurzfristig) ökonomische Effekte. Dies wird dadurch bestätigt, dass $28 \%$ der Unternehmen eine deutliche Stärkung inrer Wettbewerbsposition innerhalb der nächsten drei Jahre erwarten. Auch in bezug 
auf die Umsatzentwicklung steigen die Erwartungen der Unternehmen deutlich an. Dies deutet auf einen generellen Unterschied zwischen den Organisationstypen hin. Während bei den Universitäten die Wirkungen kurzfristig (d.h. während bzw. kurz nach dem Projekt) als besonders stark eingeschätzt werden und dann die zukünftigen Erwartungen abnehmen, ist es bei den Unternehmen genau umgekehrt. Während gerade die „harten“ Wirkungen (wie Erschließung neuer Märkte, Umsatzsteigerungen oder Kostenreduktion) am Anfang (Wirkung bereits eingetreten) eine geringe Häufigkeit aufweisen, steigen sie dann in Hinblick auf die zukünftigen Erwartungen deutlich an.

\subsubsection{Additionalität}

Abbildung 4-4: Additionalität der Rahmenprogramm-Projekte
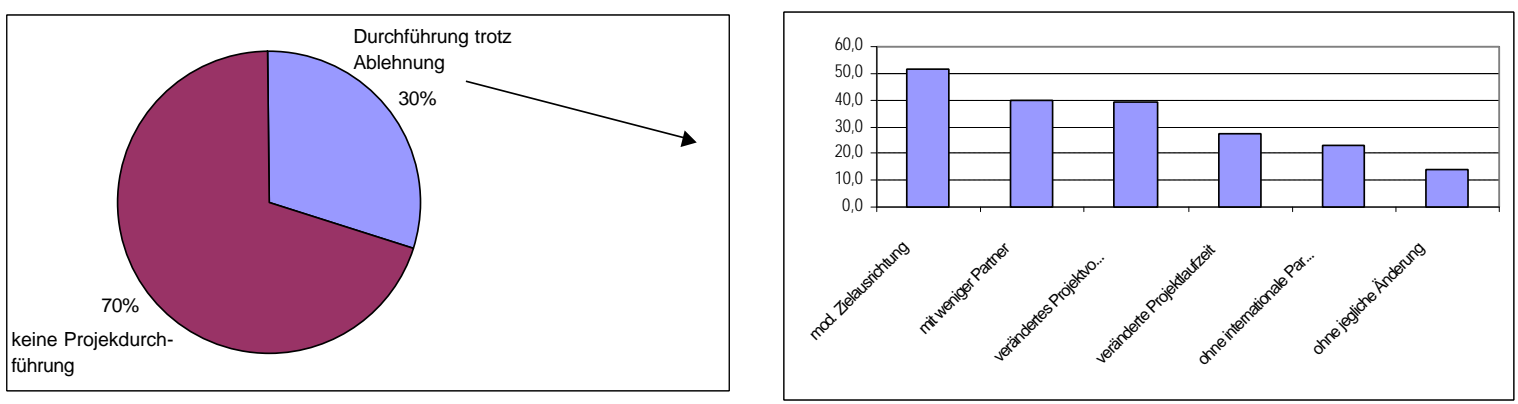

Quelle: Schibany et al. (2001)

Das Konzept der Additionalität ist im europäischen Kontext von zentraler Bedeutung und stellt im wesentlichen die Analyse des Anreizeffektes von öffentlicher Förderung von Forschungsaktivitäten dar. Hintergrund für das Interesse seitens der öffentlichen Förderstellen ist die Befürchtung, dass öffentliche Fördergelder einen Substitutionseffekt für private Investitionen darstellen, welche von geförderten Unternehmen ohnehin durchgeführt worden wären. Dies würde der Intention der öffentlichen Hand entgegenlaufen, nämlich potentielles Marktversagen zu korrigieren.

Vorhandene Studien zeigen in der Regel sehr hohe Werte für die Additionalität der Rahmenprogramme, was nicht zuletzt auch das Charakteristikum eines europäischen Projektes (multinational, mehrer Partner, etc.) widerspiegelt. In Schibany et al. (2001) wurde in diesem Zusammenhang auch die Frage gestellt, ob das Projekt ohne EU-Mittel anders durchgeführt worden wäre.

- Für Österreich gaben $70,1 \%$ der Beteiligten an, dass sie bei Projektablehnung das einschlägige Forschungsprojekt nicht weiter verfolgt hätten. Dies spricht für eine sehr hohe Additionalität der EU-Rahmenprogramme. Eine Ursache für diese hohe Additionalität liegt sicherlich in der Tatsache begründet, dass aufgrund der komplexen Projektorganisation der Aufwand zur Abwicklung des Projektes ohne EU-Förderung als zu hoch erachtet wird und das Konsortium ohne Förderung daher gänzlich auf das Projekt verzichten würde.

- Von den $30 \%$ der Antwortenden, die das Projekt auch ohne EU-Förderung weitergeführt hätten, geben nur $14 \%$ an, dass sie das Projekt ohne jegliche Änderungen durchgeführt hätten. Dies unterstreicht zusätzlich das hohe Ausmaß an Additionalität, das durch die EU-Rahmenprogramme erzielt werden kann. Jene, die das Projekt mit Modifikationen weitergeführt hätten, nennen vor allem eine modifizierte Zielausrichtung (52\%) und eine Verringerung der Zahl der Projektpartnerlnnen sowie ein verändertes Projektvolumen (jeweils $40 \%$ ). Der Mittelwert der Veränderung des Projektvolumens liegt bei $-47 \%$. Das Projekt wäre somit ohne EU-Förderung nur in einem stark reduzierten Ausmaß weiter verfolgt worden. 


\subsection{Die österreichische Beteiligung an EUREKA}

\subsubsection{Eckdaten der österreichischen Beteiligung}

EUREKA wurde 1985 von 17 westeuropäischen Ländern und der Europäischen Union gegründet. Heute zählen neben den EU und EFTA-Staaten auch ein Grossteil der osteuropäischen Staaten zu den mittlerweile 29 EUREKA Mitgliedern. Das allgemeine Ziel von EUREKA ist die Stärkung der Wettbewerbsfähigkeit der europäischen Industrien auf dem zivilen Weltmarkt.

Seit Bestehen des EUREKA-Programms (1985) haben sich österreichische Partnerlnnen an insgesamt 249 Projekten beteiligt. Diese Projektzahl umfasst die abgeschlossenen, laufenden sowie auch die vorzeitig abgebrochenen Projekte. Von den ca. 2000 EUREKA Projekten ist Österreichs somit an über $12 \%$ beteiligt. Für den Zeitraum 1995-99 zeigt Abbildung 4-5 die Anzahl der österreichischen Projekte in Relation zu der Gesamtzahl aller EUREKA-Projekte. Von den insgesamt 803 Projekten, die in diesem Zeitraum begonnen wurden, war bzw. ist Österreich an 89 Projekten beteiligt. Dabei zeigt das Jahr 1995 mit dem Beitritt Österreichs zur EU den höchsten Anteil aller gestarteten Projekte, der sich in den nachfolgenden Jahren auf einen Anteil von ca. $10 \%$ aller begonnenen Projekte senkt.

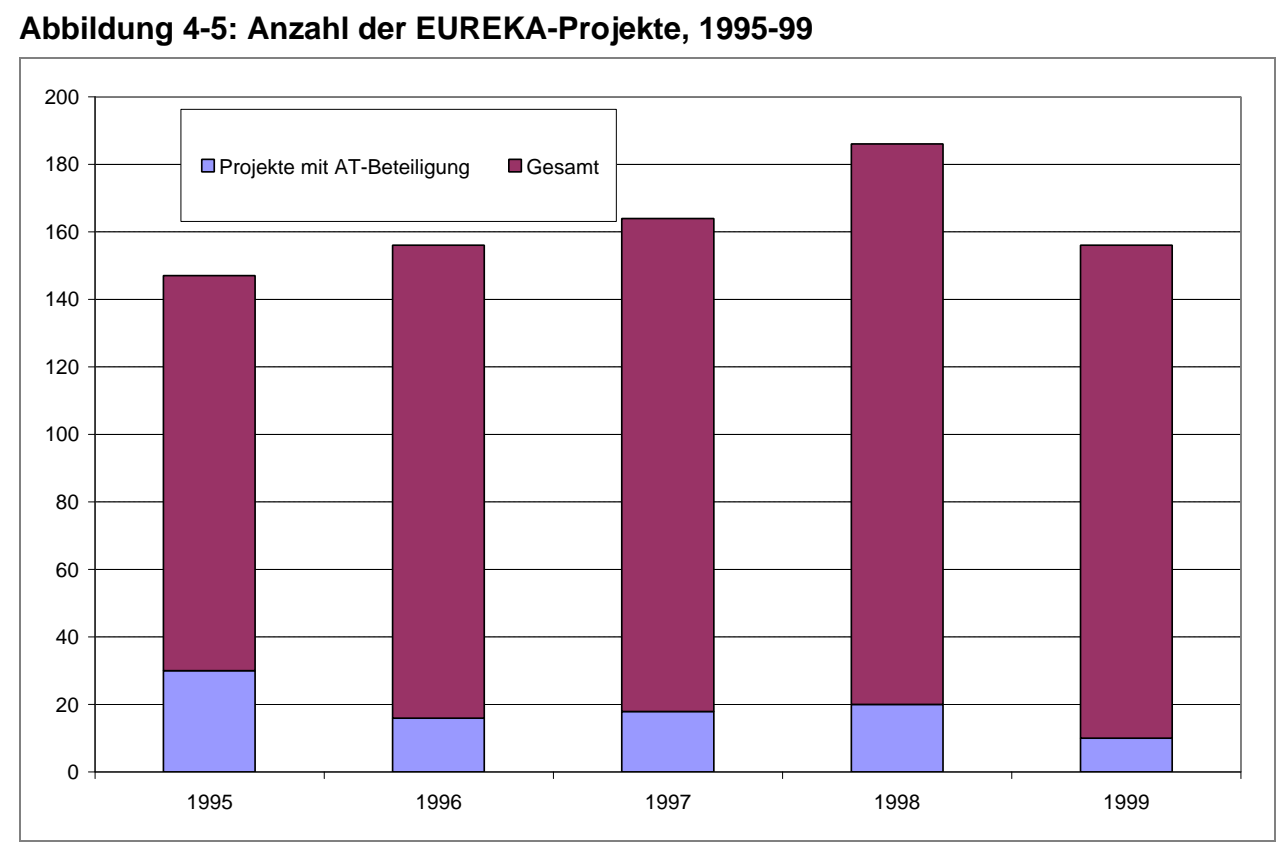

Quelle: BIT

Nach jüngsten Zahlen ${ }^{14}$ beträgt die österreichische Beteiligung immer noch etwas mehr als $10 \%$. Dies entspricht etwa der Performance von Finnland (113 Projektteilnahmen) und liegt etwas über dem EUREKA-Mittelwert. Hinzu kommt, dass es sehr große Unterschiede zwischen den EUREKA-Ländern gibt: In den letzten 5 Jahren haben 11 Länder (von 29) mehr als $80 \%$ aller EUREKA-Projekte generiert $^{15}$. Österreich gehört zu dieser Gruppe von sehr aktiven Ländern.

In den letzten Jahren hat sich die Gewichtung von strategischen Großprojekten mit hohen Kosten und langer Dauer - durchschnittlich 523 Mio. ATS / 70 Monate - zu marktnahen, kürzeren und kleineren Projekten mit Durchschnittskosten von 27,5 Mio. ATS und einer Durchschnittsdauer von 24 Monaten verschoben. Dies spiegelt auch die zunehmende Beteiligung von KMU in den letzten Jahren wieder.

\footnotetext{
${ }^{14}$ Stand 4/2001; Quelle: EUREKA-Intranet

${ }^{15}$ EUREKA Document E/N3-3a,S.2 Spanish Chairmanship, NPC-Meeting Nr.3, 4-5 April 2001
} 
Die folgende Abbildung 4-6 zeigt die Zahl der Organisationen nach Organisationstyp in 1998 laufenden Projekten. Dabei zeigt sich, dass EUREKA vor allem Industrieunternehmen anspricht. Von den fast 3.000 Teilnehmern (Unternehmen und Forschungseinrichtungen), waren zwei Drittel Industrieunternehmen, wobei $61 \%$ der Unternehmen KMU waren (das sind $42 \%$ aller Teilnehmer). Weiters kommen nahezu $25 \%$ aller Teilnehmer aus zwei der größeren Länder (Deutschland, Frankreich), gefolgt von dem Vereinigten Königreich, Spanien, der Schweiz und den Niederlanden, was insgesamt etwa die Hälfte der Teilnehmer ausmacht.

\section{Abbildung 4-6: Zahl der Organisationen in EUREKA Projekten, 1998}

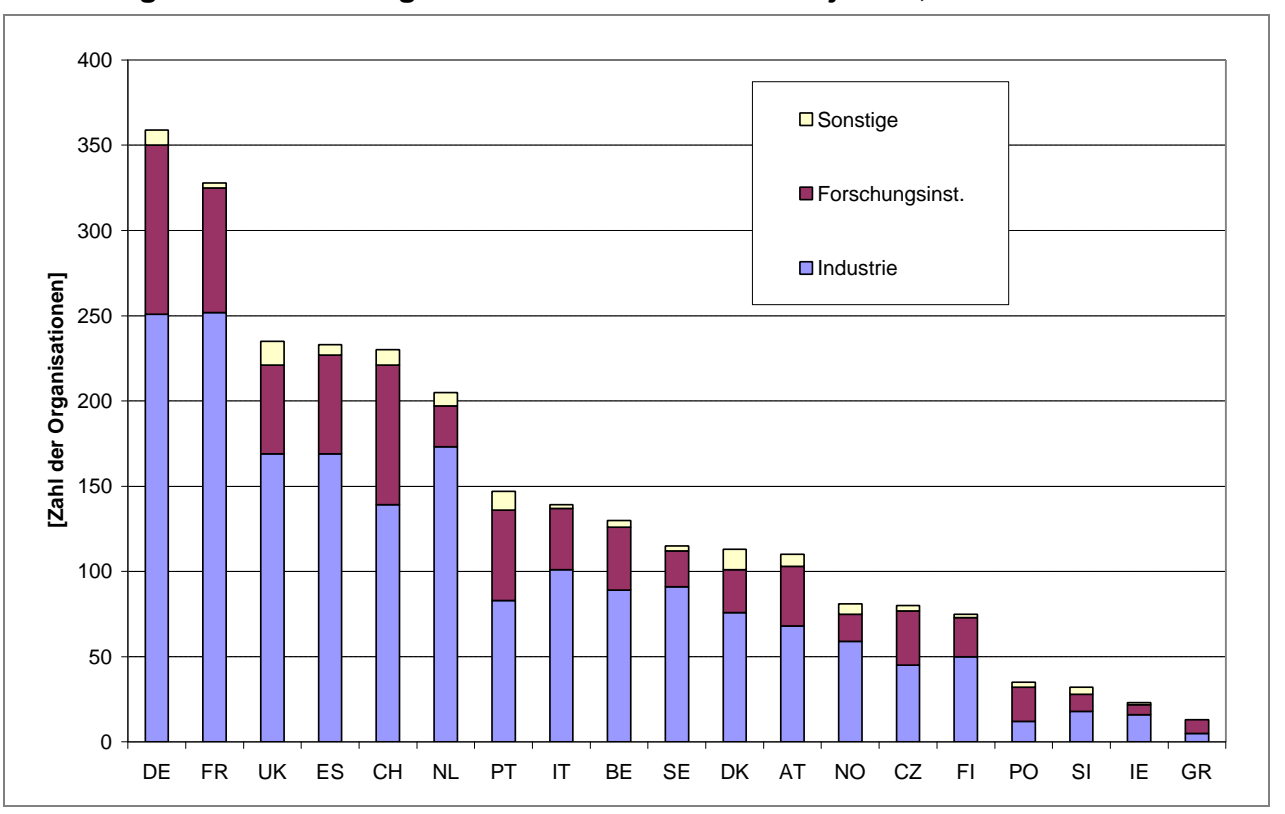

Quelle: EUREKA Jahresbericht 1999

Österreich war 1998 mit insgesamt 110 Organisationen in EUREKA Projekten vertreten, wovon 68 Unternehmen (davon $44 \mathrm{KMU}$ ) und 35 Forschungsinstitutionen (davon 24 Universitäten) waren. Ein wesentliches Charakteristikum von EUREKA ist der „bottom-up“-Ansatz, bei dem den Projektpartnern die Wahl des Projektinhalts, des Projektumfanges, der Zeitpunkt des Projektbeginns sowie die Partnerwahl überlassen bleibt. Neben der Generierung von Projektideen seitens der Unternehmen bzw. Forscher selbst, unterscheidet sich auch die Förderpraxis von den RP. EUREKA-Projekte können mit nationalen F\&E-Förderungen gefördert werden. Anträge werden über die nationalen F\&E-Fördermodelle abgewickelt. In Österreich decken die - seit dem Jahr 2000 eigens gewidmeten - EUREKAMittel des FFF (ca. 80 Mio. ATS für Zuschüsse und Darlehen zur Förderung von österreichischen EUREKA-Beteiligungen p.a.) etwa 80 \% des einschlägigen Fördervolumens ab.

Das durchschnittliche österreichische EUREKA-Fördervolumen beträgt aktuell jährlich 8,7 Mio. Euro; damit werden jährlich rund 30 österreichische Projektbeteiligungen finanziert. Bei einem durchschnittlichen Finanzvolumen von EUREKA-Projekten in der Höhe von 2,5 Mio. Euro ${ }^{16}$ bilden 30 österreichische Projekte ein Gesamtvolumen von 75 Mio. Euro, was einer Förderquote von etwa 1:8 entspricht.

\footnotetext{
${ }^{16}$ EUREKA-Dok. E/N3-2c, S.9
} 


\subsubsection{EUREKA und das Rahmenprogramm}

Die nachstehende Tabelle 4-2 bietet einen Vergleich zwischen der Beteiligung an EUREKA und am Rahmenprogramm für einige rezente Jahre:

Tabelle 4-2: Österreichische Beteiligung an EUREKA und am 4. Rahmenprogramm im Vergleich

\begin{tabular}{lcc}
\hline & $\begin{array}{c}\text { EUREKA }^{*} \\
(\mathbf{1 9 9 5 - 1 9 9 9 )}\end{array}$ & $\begin{array}{c}\text { 4. Rahmenprogramm } \\
\text { (1994-98) }\end{array}$ \\
\hline Anzahl der Projekte gesamt & 803 & 21.715 \\
Anzahl Projekte mit österr. Partner & 89 & 1.444 \\
Anteil in \% & 11,0 & 6,6 \\
Anzahl der österr. Projekt-Koordinatoren & 44 & 270 \\
Anteil in \% & 5,4 & 1,2 \\
$\varnothing$ Anzahl der Beteiligungen pro Projekt (mit AT-Beteiligung), & 6,1 & $8,3^{* *}$ \\
$\varnothing$ Anzahl beteiligter Länder pro Projekt (mit AT-Beteiligung) & 3,3 & $5,2^{* *}$ \\
\hline Quellen: Für EUREKA: Documents E/N3-3a, E/N3-2c Spanish Chairmanship, NPC-Meeting Nr.3, 4-5 April 2001; Für RP-4: vgl. & \\
$\quad$ Schibany et al. 2001, Key figures der DG Research, BIT; RP inkl. Euratom & \\
$\quad$ * Ohne Cluster- und Umbrellaprojekte, N=64 &
\end{tabular}

Die Indikatoren zur österreichischen Beteiligung an EUREKA sind durchaus vergleichbar mit denen der Beteiligung am 4. Rahmenprogramm; in einigen Aspekten ist die österreichische Performance in EUREKA klar besser:

- Österreichische Partner sind an $11 \%$ aller EUREKA-Projekte beteiligt (RP4: 6,6 \%), 5,4\% aller EUREKA-Projekte werden von einem österreichischen Partner koordiniert (RP4: 1,2 \%). Bei diesem Vergleich muss zudem noch berücksichtigt werden, dass die prozentuellen Österreich-Anteile bei EUREKA auf eine größere Grundgesamtheit von 29 Ländern berechnet werden.

- Ein Vergleich zwischen EUREKA und dem 4. Rahmenprogramm zeigt auch, dass beim Parameter „durchschnittliche Anzahl der Beteiligungen pro Projekt" EUREKA-Projekte mit 6,1 Partnern zwar unter der durchschnittlichen Beteiligung im 4. RP liegen, dennoch aber eine relativ breite Beteiligungsbasis aufweisen. Dies ist umso bemerkenswerter, als trotz der Marktnähe von EUREKA Projekten, eine nicht unbeträchtliche Anzahl von Kompetenzen zusammenwirken, was auf einen hohen Grad an Forschungsdynamik und Lerneffekten hinweist.

- An EUREKA-Projekten sind Partner aus durchschnittlich 3,3 Ländern beteiligt, im 4. Rahmenprogramm dagegen aus durchschnittlich mehr als 5,2 Ländern. Dies lässt interessante Schlussfolgerungen auf die Qualität von EUREKA Projekten zu, da die Förderungsentscheidungen jeweils auf nationaler Ebene erfolgt. Somit sind bei jedem Projekt durchschnittlich 3,3 nationale Förderstellen involviert mit jeweils unabhängigen Begutachtungen. Dabei ist es zwangsläufig so, dass die nationalen Förderstellen sich in ihrer Begutachtung nicht auf jenen Projektteil beschränken können, den der heimische Projektpartner bestreitet, sondern die Förderstellen überprüfen klarerweise die Qualität des Gesamtprojektes. Zusätzlich erfolgt die Zuerkennung des EUREKA-Status für ein F\&E-Projekt nach Prüfung der relevanten Kriterien auf Basis der Vorschläge nationaler Projektkoordinatoren.

Seit kurzem produziert das EUREKA-Büro eine sogenannte "Competitiveness Matrix“"17, in der auf Basis einer ex-ante Evaluierung die Projektvorschläge nach zwei Parametern (technologischer Innovationsgrad und wirtschaftliche Leistungsfähigkeit) beurteilt werden. Für beide Parameter wurden

\footnotetext{
${ }^{17}$ EUREKA Doc. E/N3-2c, S. 8
} 
spezifische Kriterien anhand eines Punktesystems bewertet. Die folgende Abbildung 4-7 zeigt das Resultat der Bewertung von Projekten mit österreichischer Beteiligung und der anteilsmäßigen Zuordnung zu den jeweiligen Quadranten.

Abbildung 4-7: Competitiveness Matrix von Projekten mit österreichischer Beteiligung

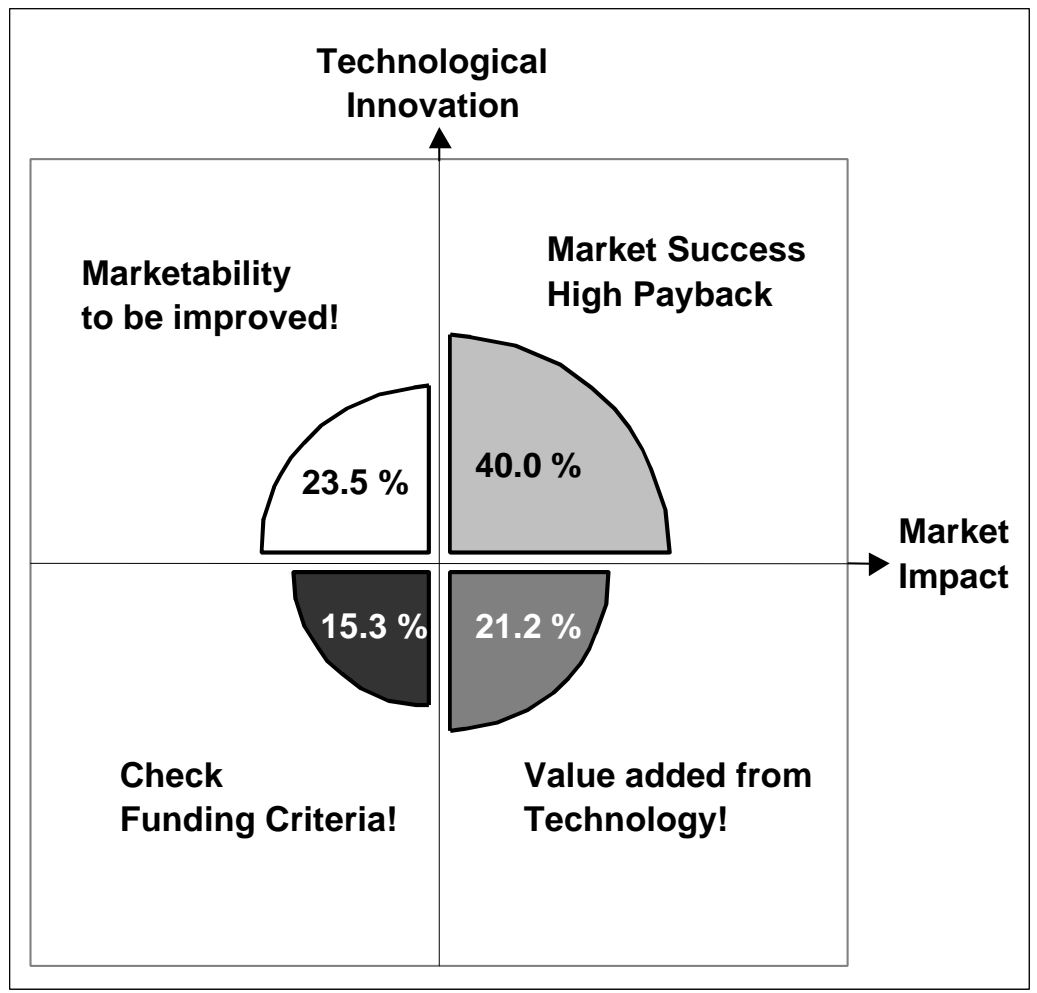

Quelle: BMVIT, Berechnungen von tip

Aus dem Sample von 85 Projektgenehmigungen weist lediglich ein Anteil von 15,3\% einen deutlich unterdurchschnittlichen Innovationsgrad sowie unterdurchschnittliche Verwertungsmöglichkeiten auf. Der Rest teilt sich auf die restlichen drei Quadranten auf. $40 \%$ aller EUREKA Projekte mit österreichischer Beteiligung sind, gemessen an den beiden Parametern, sowohl technologisch überdurchschnittlich innovativ als auch wirtschaftlich verwertbar.

Zusammenfassend lässt sich somit sagen, dass das gegenwärtige Zusammenspiel von rein nationalen Begutachtungs- und Auswahlverfahren gut funktioniert, da auf einer zweiten Evaluierungsebene die Competitiveness Matrix zeigt, dass in EUREKA mehrheitlich technologisch anspruchsvolle und marktorientierte F\&E-Projekte gefördert werden. 


\subsection{Die österreichische Beteiligung an COST ${ }^{18}$}

\subsubsection{COST im Überblick}

Österreich ist seit 1971 Mitglied der europäischen Forschungsinitiative COST, die 32 Mitgliedstaaten (15 EU- und 17 Nicht-EU-Mitglieder) sowie Israel umfasst. Gleichzeitig eröffnet COST Kooperationen mit Forschungsinstitutionen aus Drittstaaten wie Russland, Japan, Australien, Kanada und den U.S.A. und ist ein wichtiges Forum für die Zusammenarbeit mit dem Donauraum. 40.000 Wissenschaftler arbeiten in COST als einem Netzwerk zusammen. Besonders wichtig ist COST für Nachwuchswissenschaftler zur Heranführung an internationale Kooperation und kurzfristigen Forscheraustausch im Rahmen der Short Term Scientific Missions. Das im Rahmen von COST aufgebrachte Forschungsvolumen beträgt 2 Mrd Euro pro Jahr.

Die Zahl der Mitgliedstaaten ist seit der Gründung kontinuierlich gestiegen:

\begin{tabular}{|l|l|l|}
\hline 1971 & Gründung von COST & 19 Mitgliedstaaten \\
\hline 1991 & Ministerkonferenz von Wien & 25 Mitgliedstaaten \\
\hline 1997 & Ministerkonferenz von Prag & 28 Mitgliedstaaten \\
\hline 1999 & COST-Beschluss von Sliema, Malta & 32 Mitgliedstaaten \\
\hline 2000 & COST-Beschluss von Helsinki & $32+$ Israel (Sonderstatus) \\
\hline
\end{tabular}

COST fördert wissenschaftlich-technische Forschung im vorwettbewerblichen Bereich auf Grundlage gemeinsamer Koordination, jedoch auf Basis nationaler Finanzierung. Die Projekte umfassen ein Spektrum von der Grundlagenforschung bis zur industriellen Anwendung, wobei besonders multi- und interdisziplinäre Bereiche angesprochen werden.

Die „variable Geometrie“ der COST-Zusammenarbeit ermöglicht eine Beteiligung der Mitgliedstaaten nach dem „à la carte-Prinzip“: es besteht freie Wahlmöglichkeit der Beteiligung an den einzelnen Forschungsaktionen. Das hohe Interesse der Mitgliedstaaten und die lebendige Wissenschaftskooperation lässt sich eindrucksvoll an der ständig gewachsenen Zahl der Forschungsaktionen belegen:

Bei der Gründung 1971 wurden 7 COST-Aktionen beschlossen, 1990 wurden 42 Aktionen durchgeführt, im Jahr 2000 bereits rd. 150 Forschungsaktionen.

COST stellt eine Infrastruktur zur Verfügung, die es Wissenschaftlern aus ganz Europa ermöglicht, ihre Expertise auszutauschen und miteinander effizient zu kooperieren. Dies ist besonders für junge Forscherteams attraktiv, denen die Flexibilität von COST entscheidende Vorteile bietet: Offenheit für neue Impulse (bottom up approach), Workshops, "Short Term Scientific Missions" und zunehmend weltweite Kooperationen.

\footnotetext{
${ }^{18}$ COST - Coopération européenne dans le domaine de la recherche scientifique et technique - Europäische Zusammenarbeit auf dem Gebiet der wissenschaftlichen und technischen Forschung
} 


\subsubsection{Beteiligung Österreichs an COST}

Die kontinuierlich gestiegene Beteiligung Österreichs an COST ist prozentuell so hoch wie an keinem anderen Forschungsprogramm: (Quelle: COST-Jahresberichte, herausgegeben vom BMVIT)

1996/97: $\quad 51 \%$

1997/98: $\quad 52 \%$

1998/99: $\quad 70 \%$

1999/2000: $90 \%$

Besonders hervorzuheben ist die bedeutende Rolle österreichischer Wissenschaftler auf dem Gebiet der Nanowissenschaften und die starke Beteiligung an Aktionen auf medizinischem und chemischpharmazeutischem Gebiet, im Bereich der Telekommunikation, im Verkehrsbereich und auf dem Gebiet der Holzverarbeitung und Papierindustrie.

Die erfolgreiche COST-Kooperation bedeutet auch für Österreich eine entscheidende Steigerung des nationalen Innovationspotentials zu günstigen Bedingungen und Zugang zu den gemeinsam erarbeiteten Forschungsergebnissen von gesamteuropäischer Relevanz. Dabei erweist sich COST als besonders wertvolles Instrument für Österreich, für das die Zusammenarbeit mit mittel- und osteuropäischen Staaten besonderes Gewicht hat.

In Österreich gibt es keine Budgetposition für COST-Aktionen, wie es in manchen anderen Mitgliedstaaten der Fall ist (z.B. Schweiz oder Tschechische Republik). Die im Zuge der Teilnahme an COST-Aktionen entstehenden Projektkosten werden derzeit getragen durch:

1. Eigenmittel der Forschungseinrichtungen (insb. Universitätsinstitute)

2. Forschungsförderung durch Fonds: vor allem FWF, FFF

3. Finanzierung durch Unternehmen

4. Auftragsforschung der Fachressorts

5. Sonstige Mittel: Know-how, Forschungs- und Koordinationstätigkeit

\subsubsection{COST und das EU-Rahmenprogramm}

Das top-down orientierte Rahmenprogramm und die bottom-up Kooperation COST sind komplementär, wobei COST in seiner flexiblen Arbeitsweise den Europäischen Forschungsraum (ERA) unterstützt. Im EU-Haushalt besteht keine eigenständige Budgetlinie für COST, sondern die Infrastruktur von COST wird aus dem EU-Rahmenprogramm über dessen Budgetlinie INCO finanziert. Die Einpassung einer Forschungskooperation, die weit über die Grenzen der Union hinausreicht, in die INCOLinie hat sich infolge unterschiedlicher Strukturen und Zielsetzungen nicht bewährt. Von Österreich wird die Einrichtung einer eigenen Budgetlinie für COST im 6. Rahmenprogramm vorgeschlagen.

Bei der finanziellen Ausstattung von COST im 5. EU-Rahmenprogramm wurden die Mittel gekürzt, obwohl die Assoziationsabkommen zum RP mit den MOEL in Kraft getreten sind. Daher stehen COST aus dem 5. RP proportional weniger Mittel zur Verfügung. Es gilt nunmehr, im 6. RP durch eine moderate Anhebung der Mittel die finanzielle Ausstattung von COST so weit anzuheben, dass diese Kürzung wieder ausgeglichen wird und ein geeignetes effizientes Management sicher gestellt wird.

Um die Synergien zwischen Rahmenprogramm, EUREKA und COST sicherzustellen, wird die Beibehaltung eines Sekretariats der Europäischen Kommission befürwortet. Diese Leistung, hat die EK seit Gründung von COST übernommen und soll daher aus deren Erbringung nicht entlassen werden. Andere Finanzierungsformen würden letztlich wieder den Mitgliedstaaten zur Last fallen.

COST hat Modellcharakter für neue Kooperationen auch in den Beziehungen Europas zu anderen Erdteilen. Die jüngste Entwicklung hat gezeigt, dass vor allem Kanada, die USA und Australien ihre 
Beziehungen zu COST intensivieren und auf Aktionsebene verstärkt zum gegenseitigen Nutzen kooperieren. Dabei liefern COST-Projekte wesentliche Initialzündungen zu weiteren Entwicklungen in EUREKA und im Rahmenprogramm, zumal COST-Aktionen vor allem im vorwettbewerblichen Bereich, insbesondere der Grundlagenforschung, angesiedelt sind. Angesichts der Zielsetzung des künftigen Europäischen Forschungsraums stellt COST ein gutes Instrument zur Nutzung der vorhandenen nationalen Potentiale auf europäischer Ebene dar. Die COST-Kooperation liegt damit im Interesse der gesamteuropäischen Innovation und dient gleichzeitig der Wahrung der österreichischen Interessen in diesem Prozess. 


\subsection{Die Internationalisierung der Forschung und Entwicklung der Unternehmen}

Abbildung 4-8: Finanzierung von Unternehmens-F\&E durch das Ausland nach empfangenden Sektoren, 1998

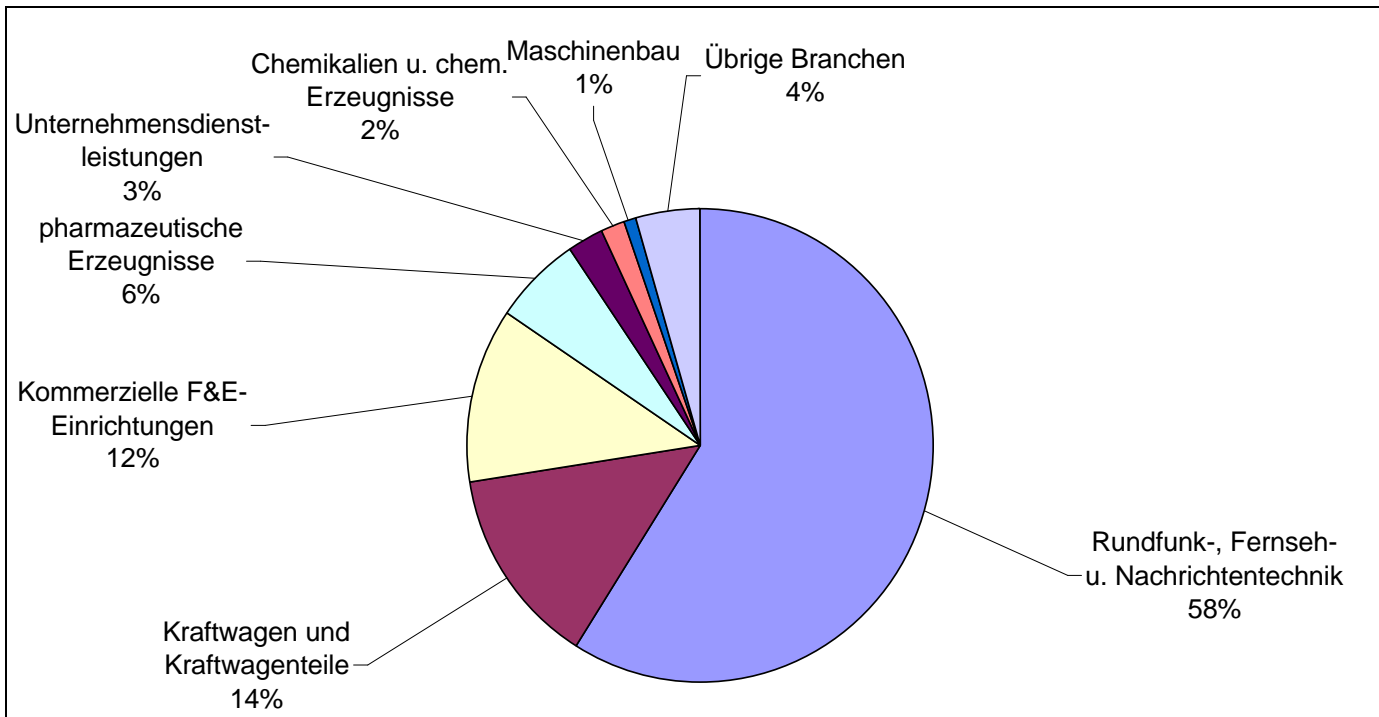

Gesamte vom Ausland finanzierte F\&E im Unternehmenssektor 1998: 7.236 Mio. ATS

Quelle: Statistik Austria (2001)

Abbildung 4-9: Finanzierung von F\&E im Ausland durch österreichische Unternehmen nach leistenden Sektoren, 1998

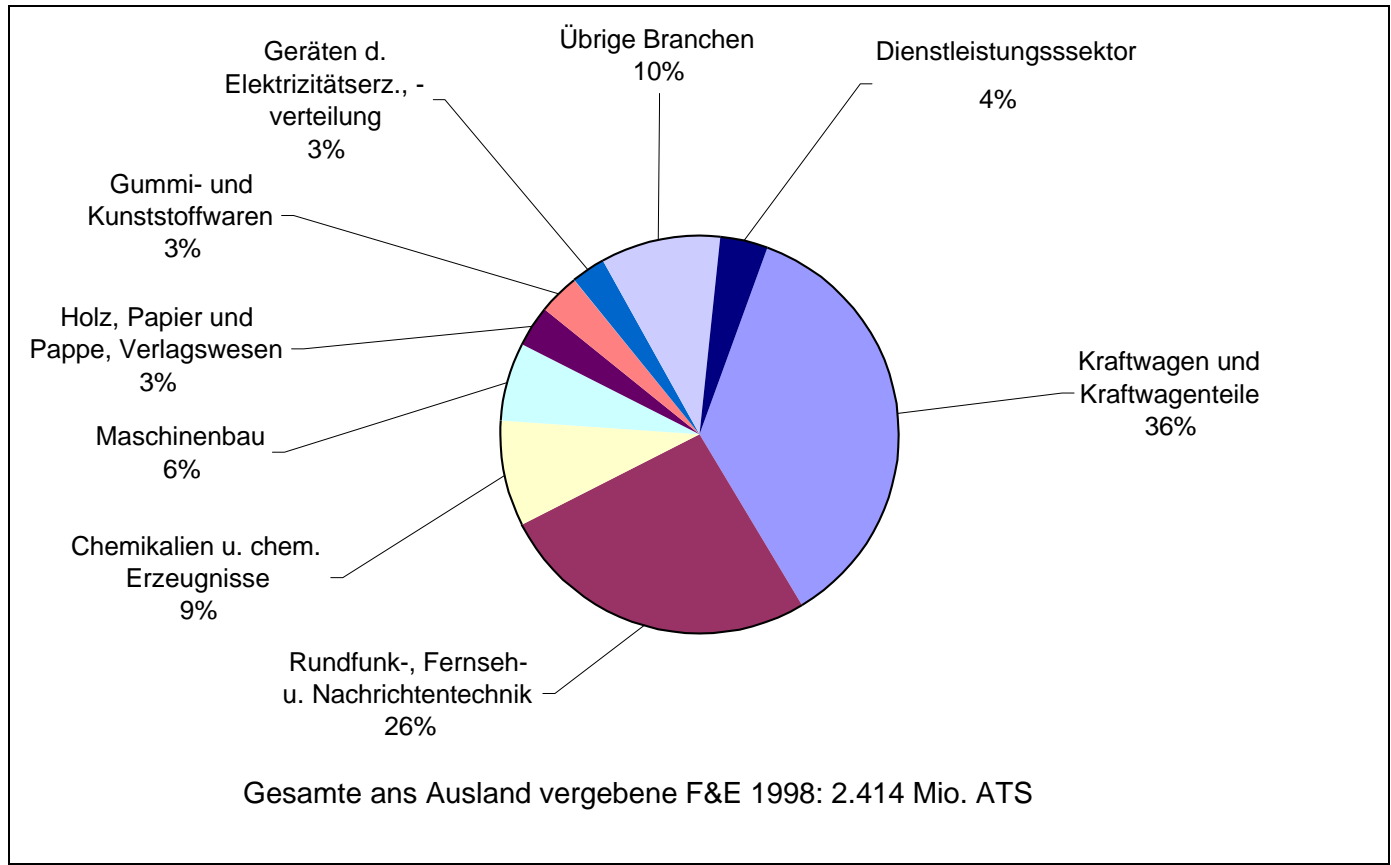

Quelle: Statistik Austria (2001)

Der Anstieg des Anteils ausländischer Finanzierung der heimischen Forschung zeigt, dass das österreichische Innovationssystem an einer Entwicklung hin zu einer stärkeren Internationalisierung der 
Unternehmensforschung teilnimmt, die in anderen OECD-Staaten schon länger beobachtet werden kann (für eine Übersicht vergleiche OECD 1998). Aufgrund der aktuellen Ergebnisse, wie sie Statistik Austria nach Revision der Forschungsstatistik ausweist, würde Österreich, was den Auslandsanteil der inländischen F\&E-Finanzierung betrifft, sogar klar alle anderen OECD-Staaten übertreffen. Gleichzeitig finanzierten österreichische Unternehmen 1998 F\&E-Aktivitäten in der Höhe von 2,4 Mrd. ATS in ausländischen Einrichtungen.

Die Internationalisierung des österreichischen Innovationssystems kann auf der Ebene der Finanzflüsse eindeutig auf die zunehmende Dezentralisierung der F\&E-Aktivitäten transnationaler Unternehmen zurückgeführt werden. Intensivere internationale Kontakte zwischen Unternehmen und Universitäten oder die Rückflüsse aus den EU-Rahmenprogrammen spielen dagegen in finanzieller Hinsicht nur eine untergeordnete Rolle. Ausländische Unternehmen, die Österreich zum Forschungsstandort gewählt haben, finanzierten 1998 im heimischen Unternehmenssektor F\&E-Aufwendungen in der Höhe von 7,2 Mrd. ATS. Die Empfänger dieser Mittel beschränken sich auf eine relativ kleine Gruppe von besonders forschungsintensiven Branchen (siehe Abb. 4-8). Österreich konnte sich in den letzten Jahren als attraktiver Standort für F\&E-Einrichtungen transnationaler Unternehmen in diesen Bereichen etablieren. Eine Reihe dieser Engagements sind das Ergebnis von Direktinvestitionen in bestehende Unternehmen. Forschung in kommerziellen F\&E-Einrichtungen (ÖNACE 73) wird beispielsweise fast ausschließlich aus eigenen Quellen sowie dem Ausland finanziert.

Transnationale Unternehmen können ihre Forschungsaktivitäten aus einer Vielzahl von Gründen dezentralisieren. Diese können etwa in Standortvorteilen wie dem Zugang zu Forschungskapazitäten, der Nähe zu bestimmten Universitäten, Forschungseinrichtungen, Unternehmensclustern oder „Communities" (z. B. in den Bereichen IT und Biotechnologie), dem Vorhandensein qualifizierter WissenschafterInnen, günstigen rechtlichen Rahmen- und Förderungsbedingungen oder „soft factors“ (z.B. hohe Lebensqualität) bestehen. Die hohe Konzentration auf forschungsintensive Sektoren kann besonders in Anbetracht der Kleinheit des österreichischen Marktes und der Nähe zu Deutschland als ein Indiz dafür gewertet werden, dass der Auslandsanteil an der heimischen Unternehmensforschung weniger aus der Anpassung von Produkten an den lokalen Markt reguliert als vielmehr eine wichtige Ergänzung der F\&E-Kompetenzen der Mutterunternehmen darstellt.

Auch die Finanzflüsse von österreichischen Unternehmen an das Ausland in der Höhe von 2,4 Mrd. ATS werden zum überwiegenden Teil von forschungsintensiven Sektoren finanziert (siehe Abb. 4-9und gehen zum überwiegenden Teil an Tochterunternehmen und verbundenen Unternehmen. Die Fahrzeugindustrie und die Rundfunk-, Fernseh- und Nachrichtentechnik sind Branchen, die sowohl wichtige Empfänger als auch wichtige Finanziers von F\&E sind. Auffällig hoch ist weiters der Anteil mittel- oder wenig forschungsintensiver Branchen wie der Textilindustrie oder der Papier- und Pappeindustrie. Auf Basis der Finanzierungsflüsse ist der Grad der internationalen Verflechtung des Dienstleistungssektors (ohne Berücksichtigung kommerzieller F\&E-Einrichtungen) wesentlich geringer als der der Sachgüterproduktion (vergleiche Abb. 4-10). 
Abbildung 4-10: Verflechtungsgrad ausgewählter Branchen mit dem Ausland, 1998

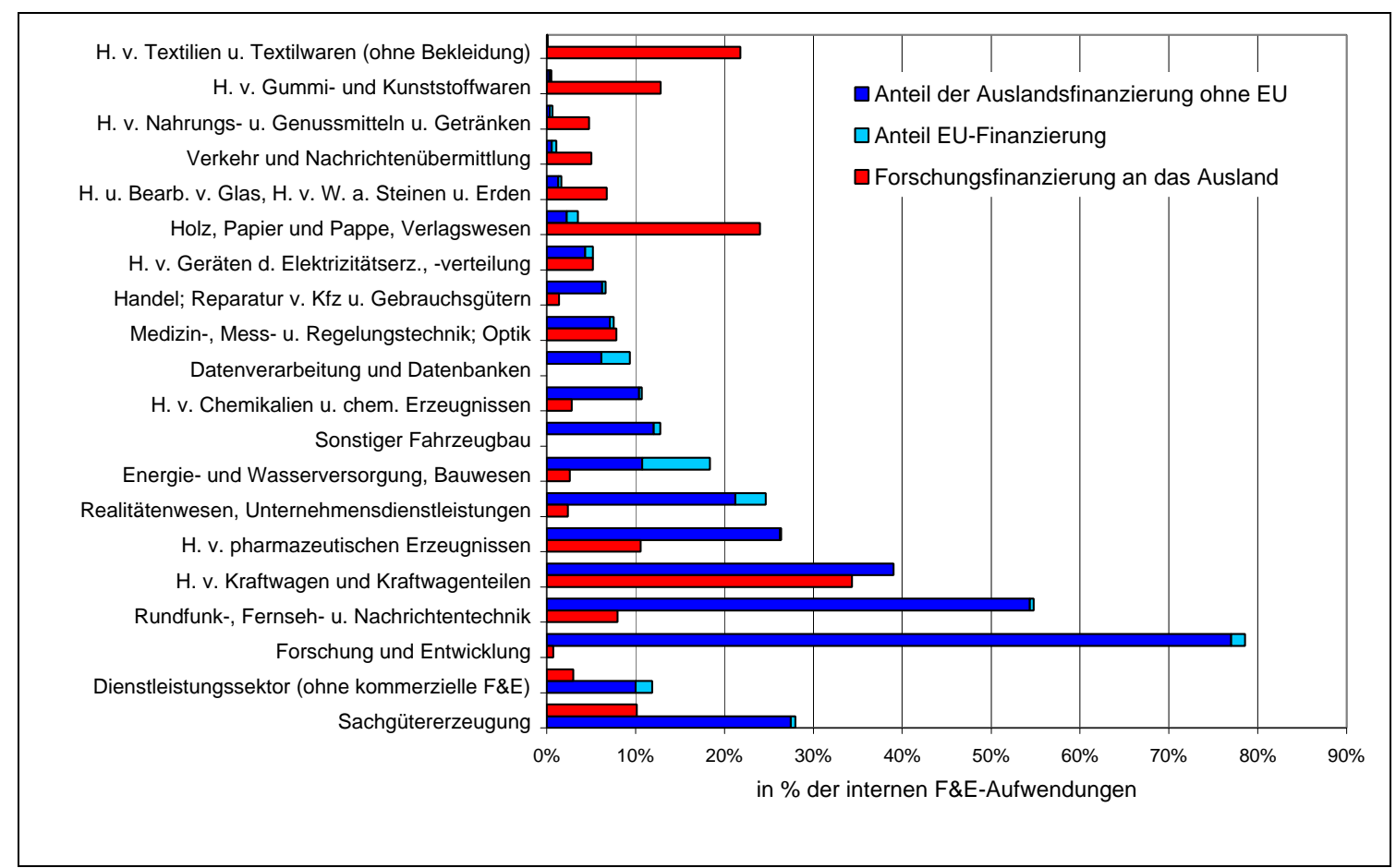

Quelle: Statistik Austria (2001) 


\section{Technologieförderung in Österreich}

Die Forschungs- und Technologieförderung war und ist ein zentrales Element der österreichischen Technologiepolitik. Andere Instrumente der Technologiepolitik, wie Regulierung, öffentliche Beschaffung, Forschungsinfrastruktur (öffentliche Forschungseinrichtungen) haben in Österreich nicht den Stellenwert wie in anderen Ländern.

Das österreichische Fördersystem ist in seiner Gesamtheit in den Wirkungen schwer abzuschätzen. Die bisherige Praxis der Evaluierung im System der österreichischen Technologieförderung ist - trotz einiger positiver Ansätze (vergleiche etwa die Aktivitäten der Plattform Technologie Evaluierung) immer noch lückenhaft und wird auch international als verbesserungsbedürftig gesehen (OECD, 1998). Zum einen wurden wichtige Elemente des Systems bisher noch keiner Evaluierung unterzogen, zum anderen werden Reformen oft punktuell, ohne Berücksichtigung des Gesamtsystems angegangen. Dabei ist die Effektivität von Förderungsinstrumenten - wie auch die OECD (Guellec, van Pottelsberghe 2000) gezeigt hat - nicht unabhängig von den anderen Komponenten des Förderungssystems.

Gründe für den großen Stellenwert der Technologieförderung sind zum einen die international hohe Innovationsdynamik und die daraus resultierenden neuen Aufgaben für die Innovationspolitik, zum anderen der Umstand, dass F\&E-Förderungen im Regime der EU-Beihilfenkontrolle gegenüber anderen Beihilfen bevorzugt werden. Darüber hinaus erhöhte sich, eben weil es sich um einen expansiven Bereich handelt, die Anzahl der darin tätigen Akteure international, national und auch regional.

\subsection{Direkte Förderung}

Die Förderungsdatenbank des Bundeskanzleramts FINKORD weist unter anderem Förderungsbarwerte für "unternehmensbezogene Technologieförderungen" des Bundes aus. Welcher Anteil dieser technologiebezogenen Förderungsmittel (die u.a. auch die Förderung von Technologiediffusion, Technologietransfer und Infrastrukturaufbau einschließen) auf die Förderung von Forschung und Entwicklung im engeren Sinn entfällt, ist nicht eindeutig zu bestimmen, unter anderem deshalb, weil die Zuordnung institutionell und nicht nach Fördergegenstand erfolgt.

Gemessen an diesem Indikator lag die direkte F\&E-Förderung in Österreich Anfang der 90er Jahre etwa im Durchschnitt der OECD-Staaten (Polt et al., 1999). Nachdem um die Mitte der 90er Jahre eine rückläufige Tendenz des Barwertes der Technologieförderung festzustellen war (1996: 1.334 Mill. ATS), stieg er in der zweiten Hälfte der neunziger Jahre wieder deutlich an (1999 auf 2.456 Mill. ATS). Damit wurde die Technologieförderung 1999 nur von der Regionalförderung knapp übertroffen, die aufgrund des Auslaufens der Regionalgebietskulisse 1999 außerordentlich hoch dotiert war.

Im österreichischen System der Direktförderungen herrschen traditionelle Instrumente wie Direktzuschüsse und gestützte Kredite vor. Die österreichische Technologieförderung hat in den letzten Jahren aber auch durchaus innovative Elemente hervorgebracht, die im Einklang mit neueren Erkenntnissen der Technologie- und Innovationsforschung eine Reihe von Dimensionen des Innovationsprozesses adressieren, insbesondere solche, die im Kontext des österreichischen Innovationssystems als wichtige Probleme wahrgenommen wurden. In einigen Programmen wurde

- dem Problem fehlender Information und Awareness über technologische Entwicklungen und Innovationspotentiale - insbesondere bei KMUs - Aufmerksamkeit geschenkt (etwa durch die Finanzierung der Inanspruchnahme von Beratungsleistungen - Austro-BUNT, Zertifizierung der Berater), 
- Technologietransfer und Technologiediffusion - wiederum mit einer Schwerpunktsetzung auf KMUs - unterstützt (etwa in den ITF-Programmen FlexCIM [Flexible Computer-integrierte Fertigung] und Technologietransfer - zu einer Evaluierung dieser Programme (vergleiche Geyer et al., 2000, bzw. Jörg et al, 2000),

- auf die Stärkung der Kooperation zwischen Wissenschaft und Wirtschaft ${ }^{19}$ abgezielt, etwa in den Kompetenzzentren-Programmen: Kplus, Kind/Knet, dem Impulsprogramm für kooperative Forschungsinstitute, den Kontaktprojekten zwischen Wissenschaft und Wirtschaft (für einen Überblick über diese Programme vergleiche Schartinger, Schibany, Gassler, 2000 sowie Polt, 2000),

- durch verschiedene Initiativen - vor allem auf der Länderebene - die Bildung industrieller Cluster unterstützt, um "kritische Massen" zu bilden (u.a. Automobil-Cluster in der Steiermark ${ }^{20}$ und Oberösterreich, Biotechnologie-Cluster in Wien)

- technologie-orientierte Unternehmensgründungen verstärkt gefördert: Business Angels, SeedFinancing, akademische Spin-Offs - "A[cademia]plusB[usiness]" (zur Entwicklung technologieorientierter Unternehmensgründungen vergleiche Almus et al., 2000, zur Konzeption akademischer Spin-Off-Förderung siehe Sturn, 2000).

Auch im "Förder-Design" waren einige wesentliche Innovationen zu bemerken: einschlägige internationale Experimente nachvollziehend wurden etwa durch die Technologie-Impulse-Gesellschaft (TIG) die Kompetenzzentren-Förderungen des Kplus-Programms in einem wettbewerblichen Verfahren vergeben, das u.a. die Selektion von "richtigen" Projekten auch unter unvollständiger Information ermöglichen sollte.

\subsection{Steuerliche F\&E-Förderung}

Eine eher weniger beachtete Rolle nimmt die steuerliche F\&E-Förderung ein. Der Forschungsfreibetrag, der mit der Steuerreform 2000 erhöht und neu gestaltet wurde, ist das bedeutendste Instrument der indirekten F\&E-Förderung in Österreich. ${ }^{21}$

Bis zum Inkrafttreten der Steuerreform 2000 wurde zusätzlich zu den sofort als Betriebsausgaben geltend zu machenden F\&E-Aufwendungen gemäß § 4 Abs. 4 EStG ein Forschungsfreibetrag von 12 $\%$ der Aufwendungen zur Entwicklung oder Verbesserung "volkswirtschaftlich wertvoller Erfindungen" anerkannt. Ein erhöhter Forschungsfreibetrag (18\%) wurde anerkannt, wenn die Forschungsergebnisse im Wesentlichen eigenbetrieblich verwertet wurden (die Einnahmen aus der Verwertung der Erfindungen $25 \%$ der Forschungsaufwendungen des jeweiligen Wirtschaftsjahrs nicht überstiegen).

Die Reform des Forschungsfreibetrags im Rahmen der Steuerreform 2000 umfasste die folgenden Kernpunkte:

- Der Forschungsfreibetrag wurde auf generell $25 \%$ angehoben.

- "Zusätzliche" (d.h. über den Durchschnitt der letzten drei Jahre hinausgehende) F\&E-Ausgaben lukrieren einen Forschungsfreibetrag von bis zu $35 \%$.

- Die Differenzierung der Begünstigung nach dem Kriterium der Eigen- oder Fremdverwertung wurde aufgehoben.

\footnotetext{
${ }^{19}$ Zur Kooperation zwischen Wissenschaft und Wirtschaft in Österreich vergleiche auch Kap. 8. 7

${ }^{20}$ Für eine Evaluierung vergleiche Adametz et al., 2000.

${ }^{21}$ Einen Überblick über das System der steuerlichen F\&E-Förderung bietet Schneider, 2000.
} 
Im Förderungsbericht 1999 des Bundesministeriums für Finanzen werden die Steuereinnahmenausfälle aus dem Titel "Forschungsfreibetrag und Spendenbegünstigung für Wissenschaft und Forschung" im Jahr 1999 (also noch vor der Steuerreform 2000) mit 1,1 Mrd. ATS angegeben. Mit dem Wirksamwerden der Steuerreform ist eine deutliche Erhöhung der Einnahmenausfälle aus diesem Titel zu erwarten. In Relation zu den anderen Instrumenten der Forschungs- und Technologieförderung stellt der Forschungsfreibetrag eine durchaus beachtenswerte Größe dar. Die steuerliche F\&EFörderung ist im internationalen Vergleich insgesamt relativ gut ausgebaut, in einzelnen Bestimmungen aber noch verbesserungsfähig: So etwa benachteiligt die gegenwärtige Regelung u.a. technologieorientierte Start-Up-Unternehmen, die in der Anfangsphase in der Regel keine Gewinne schreiben.

\subsection{Quantifizierung der aggregierten Hebelwirkungen öffentlicher Förderungen}

\section{Wirkungen öffentlicher Förderungen auf die eigenfinanzierten F\&E-Ausgaben des Unterneh- menssektors - Die Studie der OECD (2000a)}

Eine neue empirische Studie der OECD (OECD 2000a, gestützt auf Guellec, - van Pottelsberghe, 2000) versucht mittels ökonometrischer Verfahren den Einfluss verschiedener Formen öffentlicher F\&E-Ausgaben auf die eigenfinanzierten F\&E-Ausgaben des Unternehmenssektors zu quantifizieren. Dabei wurden folgende Einflussfaktoren in Betracht gezogen:
die direkte öffentliche Förderung von F\&E im Unternehmenssektor, die indirekte F\&E-Förderung (anhand eines Index der "Generosität" der Steuerbegünstigung), die F\&E-Ausgaben des öffentlichen Sektors (öffentliche Forschungseinrichtungen) und die F\&E-Ausgaben im Hochschulsektor.

Als zusätzliche Variablen wurden die Wertschöpfung und die unternehmensseitigen F\&E-Ausgaben der Vorperiode einbezogen.

Die Studie stützt sich auf Daten für 17 OECD-Länder im Zeitraum 1981-1996. Ein besonders interessanter Aspekt dieser Studie bestand darin, dass der Einfluss der oben genannten Faktoren simultan bestimmt wurde und nicht - wie zumeist praktiziert - in Isolation (z.B. Einfluss nur der steuerlichen oder nur der direkten F\&E-Förderung auf die F\&E-Ausgaben des Unternehmenssektors).

Die Hauptaussagen der Studie (die "im Durchschnitt" der betreffenden Länder, nicht jedoch für ein einzelnes Land gelten) sind die folgenden:

Sowohl die direkte als auch die indirekte F\&E-Förderung stimulierten die eigenfinanzierten F\&E-Ausgaben des Unternehmenssektors. Ein ATS an direkter Förderung erhöhte im Durchschnitt die F\&E-Ausgaben kurzfristig um insgesamt 1,61 ATS.

Direkte Förderungen wirken insofern "nachhaltiger" als steuerliche Anreize, als sie über einen längeren Zeitraum hinweg Wirkungen auf die unternehmerischen F\&E-Ausgaben haben.

Direkte F\&E-Förderung und steuerliche F\&E-Förderung sind substitutiv: eine Erhöhung der Intensität der einen vermindert die Wirkung der anderen.

Die F\&E-Ausgaben im öffentlichen Sektor und Hochschul-F\&E scheinen unternehmerische F\&E-Ausgaben zu "verdrängen". Diesbezüglich ist jedoch anzumerken, dass die im Modell der OECD enthaltenen Zeitverzögerungen viel zu kurz sind, um den Einfluss der universitären Forschung adäquat abzubilden.

Die Studie enthält wichtige Aussagen hinsichtlich der Effektivität von F\&E-Förderungen:

Weder "zu niedrige" noch "zu hohe" direkte Förderungen sind optimal. Bis zu einer Förderungsintensität von $13 \%$ nimmt der stimulierende Effekt zu, über dieser Schwelle nimmt er ab. $\mathrm{Ab}$ einer Förderintensität von 25 \% scheinen öffentliche Förderungen die private Finanzierung zu substituieren.

Unsicherheit der Unternehmen bezüglich der künftigen Förderungspolitik (z.B. durch häufige Veränderung der Förderungsbedingungen, der Förderintensitäten etc.) vermindert deren Effektivität. Dieses Ergebnis bestätigt die Resultate früherer Studien. Aus diesem Grund ist eine längerfristige Orientierung bzw. Stabilität der Förderungspolitik zu empfehlen. 
- $\quad$ Die Effektivität eines Instruments hängt vom Einsatz der anderen Komponenten öffentlicher F\&E-Ausgaben ab.

Aus der OECD-Studie lassen sich unter anderem die folgenden in Zusammenhang mit den aktuellen Zielen der österreichischen Forschungs- und Technologiepolitik wichtigen Schlussfolgerungen ableiten:

Die öffentliche Förderung von F\&E (ob direkt oder indirekt) stimuliert tatsächlich zusätzliche unternehmerische F\&E-Investitionen.

Die Förderintensität ist jedoch nicht beliebig erhöhbar, ohne dass Effizienzverluste eintreten. Der kurzfristigen Beeinflussbarkeit unternehmerischer F\&E-Investitionsentscheidungen sind daher Grenzen gesetzt.

Die Studie bietet ein starkes Argument für die Verstetigung und Koordinierung der Förderungspolitik. Eine (etwa aus budgetären Restriktionen resultierende) kurzfristig orientierte Stop-and-Go-Politik ist ineffizient. Reformen einzelner Instrumente im System der F\&EFörderung sollten - aus Gründen der Effektivität - das Gesamtsystem der Förderung im Auge haben.

\subsection{1. Ökonometrische Abschätzung der Wirkung öffentlicher F\& E-Förderung}

Kürzlich wurde am WIFO im Auftrag des BMVIT ein erster Versuch unternommen, mittels eines ökonometrischen Ansatzes den Einfluss verschiedener Politikinstrumente auf die vom Unternehmenssektor finanzierten F\&E-Ausgaben (FUNT) in Österreich zu quantifizieren (siehe Hutschenreiter, Polt, Gassler, 2001) ${ }^{22}$. Erklärende Variablen sind, neben den unternehmensfinanzierten F\&E-Ausgaben der Vorperiode:

die direkten Technologieförderungen - DIR (Barwerte laut Finkord und WIFO-Berechnungen),

die steuerliche F\&E-Förderung gemessen durch einen Index der Generosität des Forschungsfreibetrags - B (WIFO-Berechnungen),

die forschungsrelevanten Hochschulausgaben - UNI (Statistik Austria),

die Bruttoanlageinvestitionen der Industrie (auf Basis des Investitionstests des WIFO).

Die Untersuchungsperiode erstreckt sich über zwei Jahrzehnte, nämlich auf den Zeitraum 1980 1999. Die Schätzungen des ökonometrischen Modells erfolgten in logarithmischen Differenzen (Wachstumsraten). Konkret hat das geschätzte F\&E-Investitionsmodell die folgende Form:

$\Delta F U N T_{T}=C+\lambda \Delta F U N T_{T-1}+\beta_{D I R} \Delta D I R_{T-1}+\beta_{U N I} \Delta U N I_{T-1}+\beta_{B} \Delta B_{T}+\beta_{I N V} \Delta I N V_{T}+\varepsilon_{T}$

Dabei bezeichnet $\Delta$ den Log-Differenzen-Operator: $\Delta x_{T}=\log x_{T}-\log x_{T-1}$ und $\log$ den natürlichen Logarithmus.

Die vergleichsweise langen Intervalle der österreichischen F\&E-Erhebungen beschränkten die analytischen Möglichkeiten. Die Ergebnisse der ökonometrischen Untersuchung für Österreich stimmen dennoch in verschiedener Hinsicht mit den Resultaten der OECD (Guellec, van Pottelsberghe 2000) überein. Der Vergleich der Schätzergebnisse mit jenen der OECD-Studie sollte jedoch nicht über Gebühr beansprucht werden, da sich die Untersuchungen nicht nur hinsichtlich Datenbasis und Untersuchungsperiode unterscheiden, sondern auch im ökonometrischen Ansatz. Das Ziel bestand darin, eine Vorstellung von den Größenordnungen der Wirkung von Politikinstrumenten, insbesondere Förderungen auf die unternehmensfinanzierten F\&E-Ausgaben in einem relativ kurzfristigen Kontext (Erhöhung der Forschungsquote auf $2,5 \%$ bis 2005) zu gewinnen.

\footnotetext{
${ }^{22}$ Unter Mitarbeit von S. Kaniovski (WIFO).
} 
Das Hauptergebnis besteht darin, dass sich auch in Österreich ein hochsignifikanter Einfluss der direkten Technologieförderung auf die unternehmensfinanzierten F\&E-Ausgaben zeigt (durchwegs auf 1 \%-Niveau). Die durchgeführten Berechnungen geben Hinweise auf die Größenordnung dieser Wirkung.

Die Elastizität gibt an, um wie viele Prozent sich die abhängige Variable (hier: die unternehmensfinanzierten F\&E-Ausgaben, FUNT) verändert, wenn der Wert der jeweiligen erklärenden Variablen, z.B. der direkten Technologieförderungen (DIR) um ein Prozent erhöht wird. Der marginale Effekt einer Erhöhung der Variablen DIR auf die unternehmensfinanzierten F\&E-Ausgaben ergibt sich daher aus der Multiplikation der Elastizität mit der (durchschnittlichen) Relation FUNT/DIR.

In der Basisvariante gelangt man zu dem Ergebnis, dass 1 ATS direkter Technologieförderung zusätzliche unternehmensfinanzierte F\&E-Ausgaben in Höhe von 0,59 ATS induziert (Tabelle 5-1). Der Effekt eines Förderungsschillings auf die gesamten F\&E-Ausgaben beträgt daher 1,59 ATS. Im Vergleich dazu gelangt die Studie der OECD in ihrer Basisvariante zu einem kurzfristigen Effekt der direkten F\&E-Förderung auf die gesamten F\&E-Ausgaben von 1,61 (langfristig 1,70). Berücksichtigt man, dass diesen Berechnungen für Österreich ein etwas breiterer Förderungsbegriff zugrunde liegt, so können diese Ergebnisse als durchaus ähnlich bezeichnet werden.

Tabelle 5-1: Effekt einer Erhöhung der direkten Förderung um 1 ATS

\begin{tabular}{lcc}
\hline & Österreich & $\begin{array}{c}\text { 17 OECD- } \\
\text { Länder }\end{array}$ \\
\hline Elastizitäten & 0,06 & 0,07 \\
FUNT / DIR & 9,82 & 8,71 \\
Marginaler Effekt auf die unternehmensfinanzierten F\&E-Ausgaben (FUNT) & 0,59 & 0,61 \\
Marginaler Effekt auf die F\&E-Ausgaben insgesamt & 1,59 & 1,61 \\
\hline
\end{tabular}

Quelle: OECD (2000), WIFO

Die steuerliche F\&E-Förderung wurde durch einen Index der Generosität des Forschungsfreibetrags repräsentiert. Im Beobachtungszeitraum verändert sich die "Generosität" der steuerlichen Forschungsförderung drei Mal: Einmal direkt durch die Erhöhung des Forschungsfreibetrags, zweimal durch Veränderungen des Körperschaftssteuersatzes. Die Koeffizienten sind weniger robust als bei der direkten Förderung, haben jedoch in der Regel das richtige Vorzeichen. Das heißt, eine Erhöhung der Generosität der steuerlichen Anreize für F\&E (durch eine Erhöhung des Freibetragssatzes oder der Körperschaftssteuer) führt nach unseren Ergebnissen wie erwartet zu einer Steigerung der unternehmensfinanzierten F\&E-Ausgaben.

In Zusammenhang mit der Quantifizierung der Effekte steuerlicher Instrumente der F\&E-Förderung wird in der internationalen Forschung häufig eine entsprechende Nutzen-Kosten-Relation berechnet. Diese gibt das Verhältnis der durch den steuerlichen Anreiz bei den Unternehmen induzierten F\&EAusgaben zu den dadurch verursachten Steuereinnahmenausfällen wieder (und ist damit annäherungsweise mit dem Gesamteffekt der direkten Förderung auf die gesamten F\&E-Ausgaben vergleichbar). Die höchsten bisher publizierten Schätzungen liegen in einer Größenordnung von 2, die Mehrzahl der Schätzungen aus den 90er Jahren im Bereich 1,3 bis 2,0 (siehe Hall, - van Reenen, 2000).

Die Elastizität der unternehmensfinanzierten F\&E-Ausgaben in bezug auf die forschungsrelevanten Hochschulausgaben ist in allen Spezifikationen negativ. In der Basisvariante führt eine Erhöhung der forschungsrelevanten Hochschulausgaben um einen ATS zu einer Erhöhung der gesamten F\&E-Ausgaben um 72 Groschen. Auch die Studie der OECD (2000) gelangt in dieser Beziehung zu ähnlichen Resultaten. Auch hier dominiert anscheinend der Crowding-Out-Effekt den Stimulierungseffekt. In bei- 
den Modellen erscheinen die Zeitverzögerungen jedoch viel zu kurz, um den Einfluss der universitären Forschung adäquat abzubilden.

Zusammenfassend erscheinen nach den vorliegenden Ergebnissen die Resultate der OECD hinsichtlich der Hebelwirkung von Förderungen als brauchbare Arbeitshypothese. Die Erwartung, dass ein Ausbau von Förderungen im Aggregat zusätzliche unternehmensfinanzierte F\&E-Ausgaben in gleicher Höhe nach sich ziehen, erscheint - jedenfalls in der kurzen Frist - eher hoch gegriffen, in der Öffentlichkeit zuweilen kolportierte vielfache "Hebelwirkungen" umso mehr. Dies schließt natürlich nicht aus, dass einzelne Instrumente eine stärkere Wirkung auf die unternehmensfinanzierte Forschung haben, als die hier im Durchschnitt geschätzte. 


\section{Zur technologischen Leistungsfähigkeit des Unternehmenssektors}

\subsection{Struktur und Leistungsfähigkeit nach Branchentypen}

\subsubsection{Die WIFO Branchentaxonomien}

Internationale Vergleiche von Spezialisierungsmustern in Produktion und Außenhandel zielen darauf ab, die Leistungsfähigkeit einer Volkswirtschaft anhand des tatsächlich realisierten ökonomischen Erfolges bei der wirtschaftlichen Aneignung technologischen Wissens zu messen. Voraussetzung ist eine ökonomisch interpretierbare Basis, die es erlaubt, Rückschlüsse auf die relativen Stärken und Schwächen in Bezug auf jene Bestimmungsfaktoren vorzunehmen, die im Wettbewerb über den unternehmerischen Erfolg oder Misserfolg entscheiden. Diesen Zweck erfüllt der Gebrauch von Klassifikationen, die unterschiedliche Produktionszweige oder Produktgruppen unter gemeinsamen analytischen Gesichtspunkten zusammenfassen.

Am WIFO wurden im Auftrag der Europäischen Union in den vergangenen Jahren drei neue Klassifikationen der Wirtschaftszweige entwickelt (Tabelle 6-11), die seither zu einem festen Bestandteil der Kommissionsberichte über die Wettbewerbsfähigkeit der europäischen Industrie ${ }^{23}$ geworden sind; ein Befund, der aus österreichischer Sicht positiv zu bemerken ist.

Der Grundgedanke von Taxonomie I beruht auf der Unterscheidung zwischen vornehmlich standortgebundenen Wettbewerbsvorteilen auf der Grundlage von unterschiedlichen Faktorpreisen etwa von Kapital und Arbeit einerseits, sowie vom Unternehmen selbst geschaffenen Wettbewerbsvorteilen auf der Grundlage strategischer Investitionen in immaterielle Aktiva wie Marketing und Forschung andererseits. Für Taxonomie II wurden Daten über die relativen Beschäftigungsanteile von hoch bzw. niedrig qualifizierten Beschäftigten herangezogen. Taxonomie III gliedert die Branchen der Sachgütererzeugung nach ihrer unterschiedlichen Art und Intensität in der Nachfrage nach externen Dienstleistungen.

Wie jede Typenbildung beruhen auch die neuen Klassifikationen des WIFO auf der Reduktion und Verdichtung von Information und müssen daher vorsichtig interpretiert werden. Selbstverständlich sind die in den Typen zusammengefassten Wirtschaftszweige noch immer sehr heterogen. Anhand statistischer Testverfahren lässt sich jedoch nachweisen, dass ein Großteil der beobachtbaren Variation zwischen den Branchen z.B. in der Kapital-, Forschungs- oder Werbeintensität durch die Klassifizierung erfolgreich innerhalb des jeweiligen Industrietyps isoliert werden konnte (Peneder, 2001).

\footnotetext{
${ }^{23}$ European Communities (1998), The Competitiveness of European Industry: 1998 Report, Luxembourg; European Communities (1999), The Competitiveness of European Industry: 1999 Report, Luxembourg; European Communities (2000), European Competitiveness Report 2000, Luxembourg.
} 
Tabelle 6-1: Die WIFO Branchentaxonomien

\begin{tabular}{|c|c|c|c|c|}
\hline \multicolumn{5}{|c|}{ Taxonomie I (Faktoreinsatztypen) } \\
\hline $\begin{array}{l}\text { Traditionelle Sachgü- } \\
\text { terbranchen (TS) }\end{array}$ & $\begin{array}{l}\text { Arbeitsintensive In- } \\
\text { dustrien }(\mathrm{Al})\end{array}$ & $\begin{array}{l}\text { Kapitalintensive In- } \\
\text { dustrien (KI) }\end{array}$ & $\begin{array}{l}\text { Marketing- ge- } \\
\text { stützte Industrien } \\
\text { (MGl) }\end{array}$ & $\begin{array}{l}\text { Technologie- ge- } \\
\text { stützte Industrien } \\
\text { (TGI) }\end{array}$ \\
\hline \multicolumn{5}{|c|}{ Taxonomie II (Qualifikationstypen) } \\
\hline $\begin{array}{l}\text { Branchen mit vor- } \\
\text { nehmlich ... }\end{array}$ & $\begin{array}{c}\text {.. niedrig qualifizierten } \\
\text { Beschäftigten (NQ) }\end{array}$ & $\begin{array}{l}\text {.. mittel qualifizierten, } \\
\text { blue-collar' Beschäf- } \\
\text { tigten (MBC) }\end{array}$ & $\begin{array}{l}\text {.. mittel qualifizierten, } \\
\text { 'white-collar’ Be- } \\
\text { schäftigten (MWC) }\end{array}$ & $\begin{array}{c}\text {.. hoch qualifizier- } \\
\text { ten Beschäftigten } \\
(\mathrm{HQ})\end{array}$ \\
\hline \multicolumn{5}{|c|}{ Taxonomie III (externe Dienstleistungsinputs) } \\
\hline $\begin{array}{l}\text { Branchen mit einem } \\
\text { hohen Anteil an Inputs } \\
\text { von ... }\end{array}$ & $\begin{array}{l}\text {... wissensintensiven } \\
\text { Dienstleistungen } \\
\text { (IWDL) }\end{array}$ & $\begin{array}{c}\text {... Dienstleistungen in } \\
\text { Marketing und Ver- } \\
\text { trieb (IM\&V) }\end{array}$ & $\begin{array}{l}\text {... Transport- } \\
\text { dienstleistungen } \\
\text { (ITR) }\end{array}$ & Sonstige \\
\hline
\end{tabular}

Quelle: Peneder (2001)

\subsubsection{Strukturmerkmale: Produktivität, Wachstum und Qualitätswettbewerb}

Tabelle 6-2 fasst zusammen, warum Spezialisierungsmuster innerhalb der angeführten Kategorien für die Beurteilung der techno-ökonomischen Leistungsfähigkeit eines Landes von Bedeutung sind. Es werden darin systematische Unterschiede zwischen den einzelnen Wirtschaftszweigen sowohl in Bezug auf ihre durchschnittliche Wachstumsperformance, die qualitative Differenzierbarkeit der angebotenen Güter und Leistungen sowie hinsichtlich der Arbeitsproduktivität als unmittelbarem Beitrag zur volkswirtschaftlichen Einkommensbildung bestätigt.

Wenn man die Europäische Union, Japan und die USA zusammenzählt, dann war für den Zeitraum von 1990 bis 1998 das durchschnittliche Wachstum der Konsumnachfrage in den technologiegestützten Branchen (+3,75 \% p.a.) mit Abstand am höchsten. Überdurchschnittlich hohe Wachstumsraten des Konsums begünstigten auch die Entwicklung in Branchen mit hohem Anteil qualifizierter Arbeitskräfte, in Industrien mit großer Nachfrage nach marketing- und vertriebsorientierten Dienstleistungen, sowie solchen mit hohen Inputs an wissensintensiven Dienstleistungen. Mit Abstand am geringsten war die Dynamik der Nachfrage in der Gruppe der kapitalintensiven Branchen ( $+1,48 \%$ p.a.). Allerdings stellt auch die Gruppe der traditionellen Sachgütererzeugung ( $+3,22 \%$ p.a.) unter Beweis, dass ein hohes Wachstum der Nachfrage nicht notwendigerweise auf "moderne“ Industriezweige beschränkt sein muss.

Die Produktivität der Arbeit steigt mit der Effizienz und der Menge im Einsatz zusätzlicher Produktionsfaktoren wie Anlageinvestitionen, Forschung, Werbung oder der Qualifikation der Beschäftigten (Humankapital). Insgesamt zeigt sich, dass im Jahr 1998 die Arbeitsproduktivität in den technologiegestützten Industrien am höchsten war, gefolgt von kapitalintensiven Branchen sowie Industrien mit hohem Anteil wissensintensiver Dienstleistungen. Mit Abstand am geringsten ist das Niveau der Arbeitsproduktivität in der Gruppe arbeitsintensiver Branchen, die dadurch gekennzeichnet sind, dass zusätzliche Produktionsfaktoren nur in relativ geringen Mengen zum Einsatz kommen. 
Tabelle 6-2: Wachstum, Produktivität und Unit Values nach Branchentypen: EU-Japan-USA

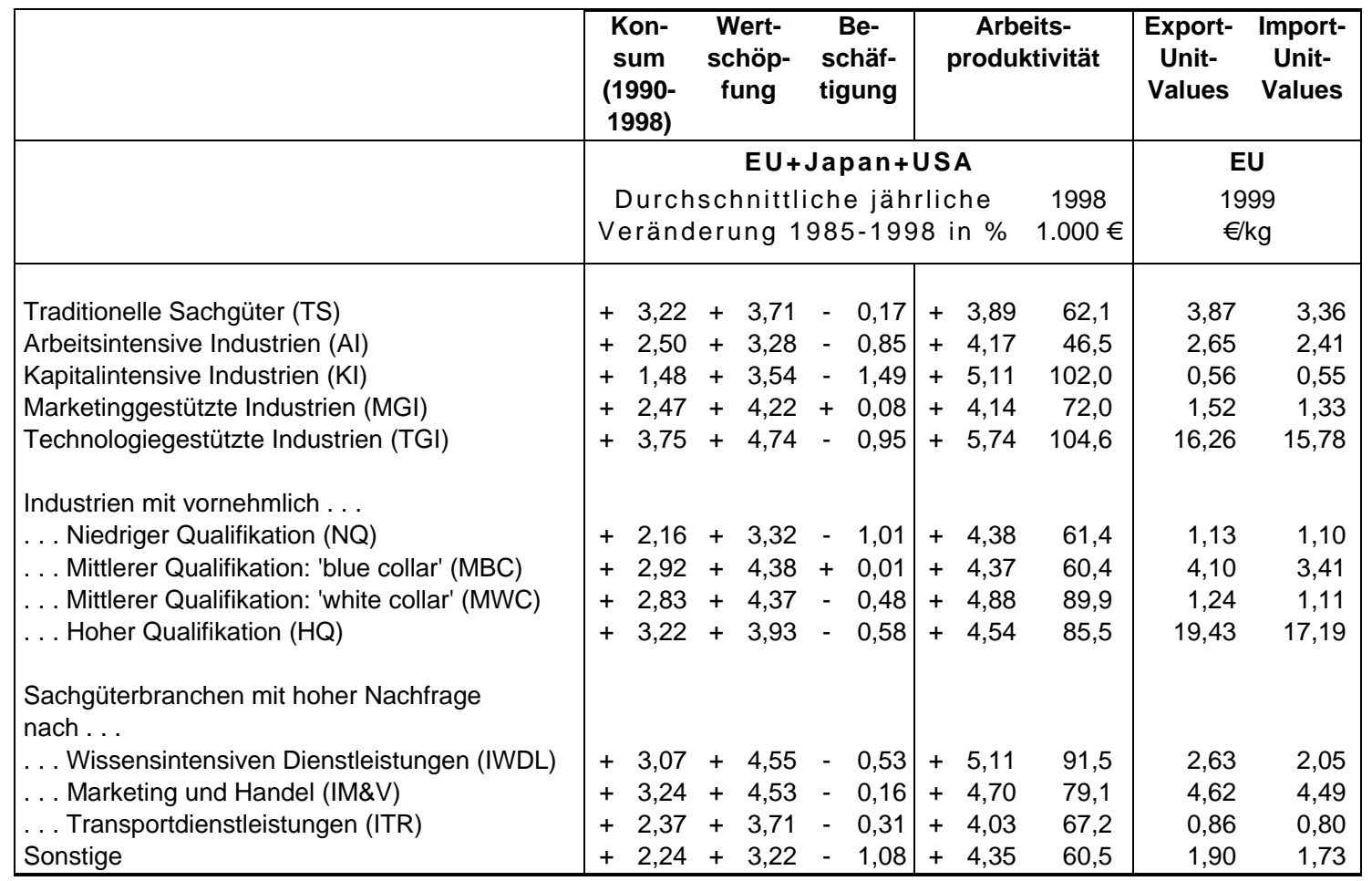

Quelle: WIFO

Vergleicht man die durchschnittlichen jährlichen Wachstumsraten von Wertschöpfung, Beschäftigung und Arbeitsproduktivität zwischen 1985 und 1998, dann bestätigen sich hohe Zuwachsraten in der Wertschöpfung v.a. für marketing- und technologiegestützte Industrien sowie für jene Branchen der Sachgütererzeugung, deren Vorleistungen durch besonders hohe Anteile wissensintensiver sowie marketing- bzw. vertriebsorientierter Dienstleistungen gekennzeichnet sind. Sowohl bei den technologiegestützten Branchen als auch bei den Produktionszweigen mit hohem Anteil wissensintensiver Dienstleistungen verhindern aber die ebenfalls sehr großen Produktivitätsgewinne eine positive Entwicklung der Beschäftigung.

"Unit Values" sind ein Maß für vertikale Differenzierung und Qualitätswettbewerb und errechnen sich aus dem nominellen Handelswert dividiert durch die physische Menge von Handelswaren, die z.B. in Gewichtseinheiten oder Stückzahlen gemessen werden. Dabei zeigt sich im Vergleich der Industrietypen, dass die Gruppen der vornehmlich technologiegestützten Industrien sowie jene mit besonders hohem Anteil hochqualifizierter Arbeitskräfte mit Abstand und signifikant höhere Unit Values aufweisen als alle anderen Gruppen.

\subsubsection{Eine österreichische Besonderheit: gute Performance in traditionellen Strukturen}

Die Beobachtung persistenter Defizite in der Spezialisierung auf moderne, wachstumsorientierte und technologisch anspruchsvolle Produktionszweige geht bereits auf die Strukturberichterstattung der 80er Jahre zurück und fand in den 90er Jahren seine Fortsetzung unter den Begriffen der „Technologielücke“, des "Struktur-Performance Paradoxon“ (Hutschenreiter und Peneder 1997, Peneder 1999) 
und zuletzt des "Nutzer-Paradoxon“ (Tichy 2001). ${ }^{24}$ Die neuen Branchenklassifikationen erlauben einen frischen Blick auf diese Befunde (Tabelle 6-1).

In der Abgrenzung nach typischen Faktoreinsatzmengen weicht das österreichische Spezialisierungsmuster von jenem der Europäischen Union v.a. in zwei Gruppen ab: Einem deutlich höheren Anteil arbeitsintensiver Industrien (20,13\% versus $15,55 \%$ in der EU) steht in Österreich ein markant niedrigerer Wertschöpfungsanteil in der Gruppe technologiegestützter Produktionszweige $(14,16 \%$ vs. $20,92 \%)$ gegenüber. Ähnlich stark ausgeprägt ist das Strukturdefizit hinsichtlich jener Taxonomie, die Branchen nach Art und Ausmaß der zugekauften Dienstleistungen unterscheidet. Bedenklich ist der auffallend geringe Anteil von Branchen mit ausgeprägter Nachfrage nach wissensintensiven Dienstleistungen (12,40 \% vs. 19,32 \%), während etwa der Anteil von Branchen mit großer Nachfrage nach Transportdienstleistungen (32,39\%) deutlich über dem EU Durchschnitt $(23,57 \%)$ liegt. Weniger stark ist das Strukturdefizit nach Qualifikationstypen ausgeprägt. Allerdings liegt auch hier der Anteil von Industrien mit besonders großen Anteilen hochqualifizierter Beschäftigter $(13,11 \%)$ unter dem Wert für die Europäische Union (16,67 \%).

Trotz dieser deutlichen Strukturdefizite zeigt sich in der gesamtwirtschaftlichen Betrachtung, dass Österreich in den 90er Jahren mit durchschnittlich 2,2 p.a. um rund 0,4 \% schneller gewachsen ist als der Durchschnitt der Europäischen Union. Und gerade die Sachgütererzeugung konnte mit durchschnittlich $+3,6 \%$ p.a. ihren realen Produktionswert um fast die Hälfte steigern. Im Vergleich mit den anderen EU-Ländern ist dies das vierthöchste Wachstum innerhalb der vergangenen Dekade. Deutlich schneller ist die Industrieproduktion lediglich in Irland ( $+10,8 \%$ p.a.), Finnland $(+5,2 \%$ p.a.) und Schweden (+4,2\% p.a.) gestiegen. Im Gegensatz zu Österreich ist in all diesen Ländern die gute Wachstumsleistung aber mit einem deutlichen Strukturwandel einhergegangen.

Zusammenfassend lässt sich festhalten, dass die österreichische Sachgüterproduktion überwiegend in traditionellen Branchen mit durchschnittlich bis geringem technologischen Anspruchsniveau tätig ist. Die positiven Befunde zur makroökonomischen Entwicklung zeigen jedoch, dass sie sich innerhalb diese Märkte sehr gut behauptet. Die möglichen längerfristigen Auswirkungen dieses Befunds für Österreich verdienen in der wirtschaftspolitischen und technologiepolitischen Diskussion mehr Aufmerksamkeit als bisher.

\footnotetext{
${ }^{24}$ Das „(Struktur-) Performance Paradoxon“ verweist auf den Umstand, dass den im internationalen Vergleich eher geringen Investitionen Österreichs in Forschung und Entwicklung eine positive langfristige Performance der österreichischen Wirtschaft gegenübersteht.

Das „Nutzer-Paradoxon“ verweist auf den Umstand, dass die Nutzer zwar am ehesten über neue innovatorische Bedürfnisse wüssten und demgemäß hinter den meisten wichtigen Innovationen stünden; zugleich aber steuerten sie den Innovationsprozess zugunsten einer Verbesserung bestehender Produkte zulasten neuer Ansätze, verhinderten also radikale Innovationen. Fazit: „Hinter dem langsamen technischen Fortschritt in Österreich steht die Scheu der Unternehmer vor größeren, wenn schon nicht radikalen Innovationen" (zitiert nach Tichy 2001, S.224).
} 
Abbildung 6-1: Anteile an der Wertschöpfung in der Sachgütererzeugung 1998 in \%

Taxonomie I (Faktoreinsatzmengen)

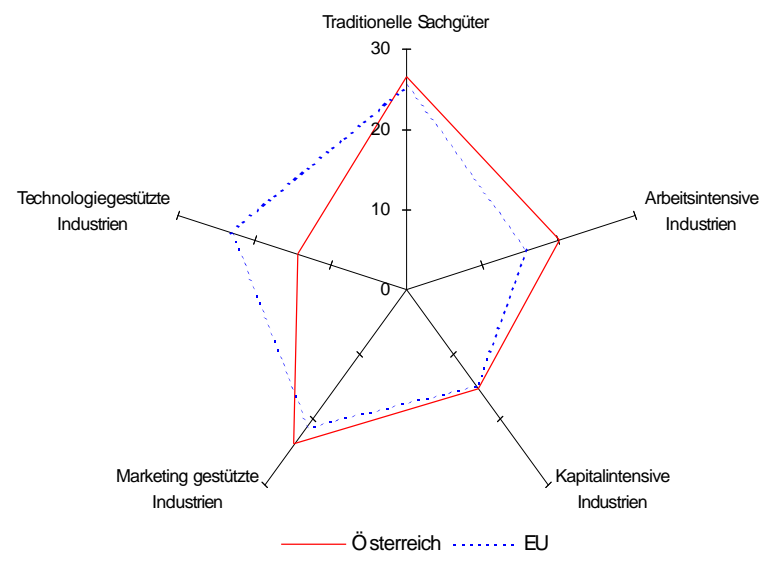

Taxonomie II (Qualifikationstypen)

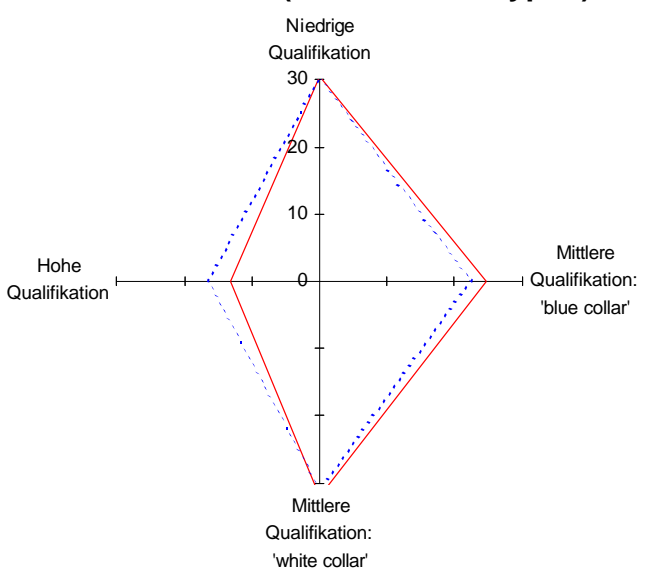

Taxonomie III (Nachfrage nach externen Dienstleistungen)

Wissensintensive

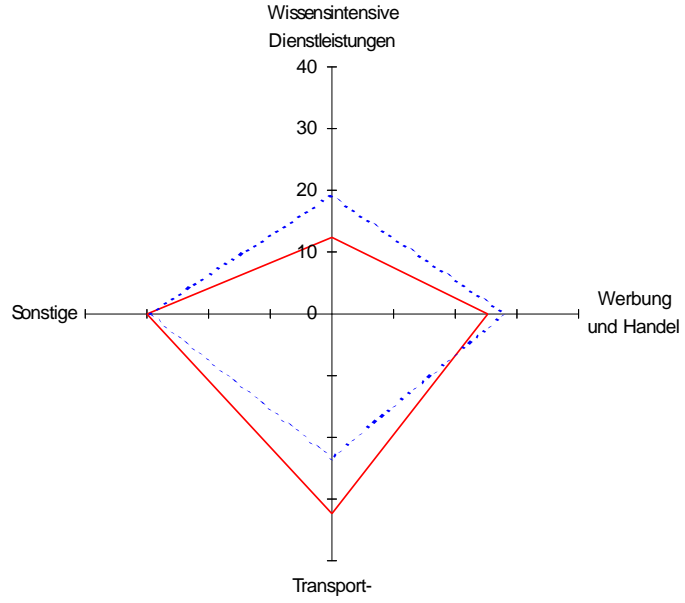

Quelle: WIFO 


\subsection{Unternehmensgründungen in Österreich ${ }^{25}$}

Nach wie vor lässt sich für Österreich im Vergleich zu Referenzregionen wie Westdeutschland und Bayern (hier steht eine vergleichbare Datenlage zur Verfügung) eine generelle Gründungsschwäche feststellen. Bezogen auf alle Gründungen weist Österreich eine niedrigere Gründungsintensität (Zahl der Neugründungen pro 10.000 erwerbsfähige EinwohnerInnen) auf. Im Zuge der 90er Jahre kann auch keine Verringerung des Abstands im Gründungsniveau zu den Referenzregionen beobachtet werden. Allerdings konnten die Gründungs-Defizite im Bereich der F\&E-intensiven Branchen des Verarbeitenden Gewerbes inzwischen aufgeholt werden, bei den F\&E-intensiven Branchen der unternehmensnahen Dienstleistungen werden die nach wie vor bestehenden Defizite durch die stetige Zunahme der Gründungszahlen seit 1993 sukzessive vermindert.

\section{Abbildung 6-2: Struktur der Unternehmensgründungen (1997/98) Österreichs im Vergleich zu Deutsch- land (alte Bundesländer)}

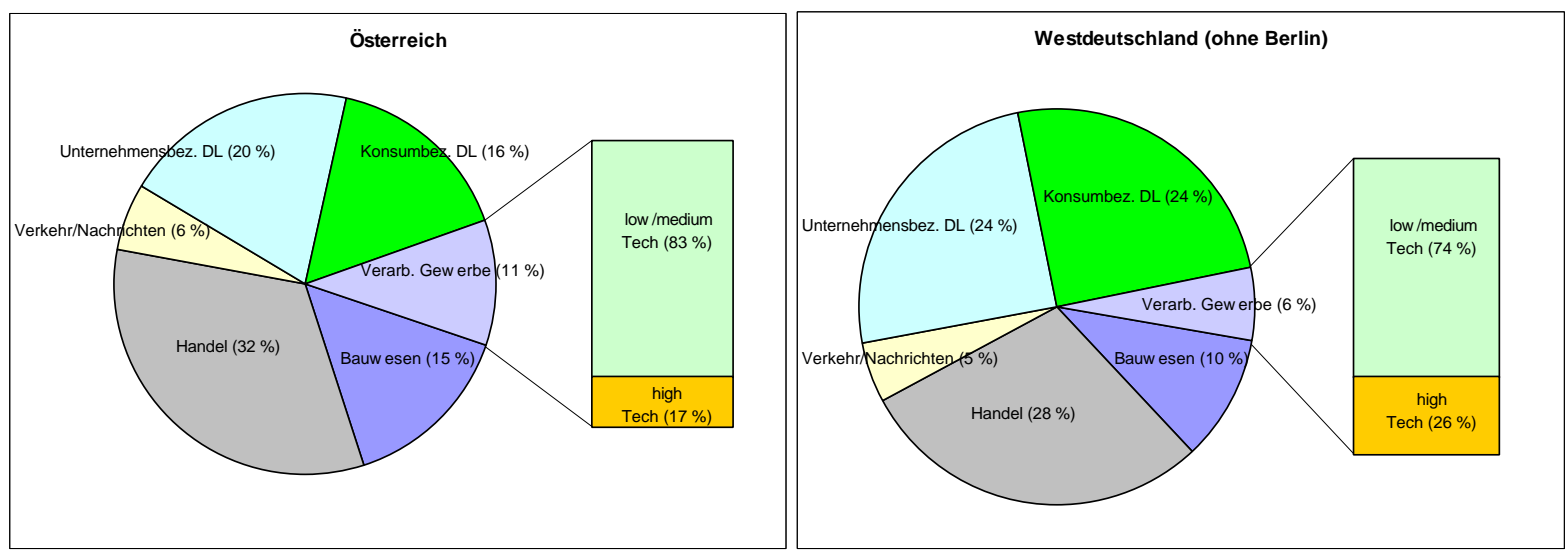

Quelle: Gründungsmonitoring ZEW/ARCS (Almus et. al 2000)

Eine Ursache für die generelle Gründungsschwäche Österreichs kann in der unverändert industrielastigen Struktur der österreichischen Neugründungen zu finden sein. In den 90er Jahren steigen die Gründungszahlen in den Branchen des Verarbeitenden Gewerbes tendenziell sogar an, während sie in Westdeutschland und Bayern laufend zurückgehen. Ende der 90er Jahre (1997/98) ist der Anteil industrieller Gründungen in Österreich fast doppelt so hoch wie in den deutschen Vergleichsräumen (siehe Abbildung 6-2). Der Anteil der neuen Unternehmen aus den - insgesamt erheblich gründungsintensiveren - Dienstleistungsbranchen ist nach wie vor relativ niedrig. Zwar steigt der Anteil der unternehmensnahen Dienstleistungen leicht an, jener der konsumorientierten Dienstleister stagniert allerdings auf niedrigem Niveau.

Die Technologieorientierung der Gründungen im Verarbeitenden Gewerbe ist zwar um einiges niedriger als in den deutschen Vergleichsräumen. Da der Anteil industrieller Gründungen in Österreich jedoch wie erwähnt deutlich höher ist als in Westdeutschland, liegt der Anteil F\&E-intensiver Gründungen an allen Gründungen in Österreich mit ca. 1,8 \% (1997/98) sogar leicht über jenen in Westdeutschland $(1,5 \%)$

Trotz dieser Industrielastigkeit der Gründungen ist die Strukturquote (berechnet als Verhältnis des Anteils einer Branche bei den Gründungen im Vergleich zu dem entsprechenden Anteil im Unternehmensbestand) des Verarbeitenden Gewerbes kleiner als eins, d.h. der Anteil des Verarbeitenden Gewerbes an den Neugründungen ist kleiner als am Unternehmensbestand. Die Strukturquote der

\footnotetext{
${ }^{25}$ Vorliegende Kurzübersicht basiert auf den zusammenfassenden Überlegungen des Gründungsmonitoring von ZEW/ARCS (Almus et al., 2000).
} 
unternehmensnahen Dienstleistungen ist erheblich größer als eins. Die Gründungen tragen somit zu einer Strukturveränderung der österreichischen Wirtschaft in Richtung moderner Dienstleistungen bei.

Neu gegründete Unternehmen tragen nicht unbeträchtlich zum Beschäftigungswachstum bei. Allerdings weist der überwiegende Teil der jungen Unternehmen kein oder nur ein sehr geringes Wachstum auf. Die Wachstumsbeiträge kommen von einigen wenigen Unternehmen, die dann jedoch beachtliche Beschäftigungswachstumsraten aufweisen (sogenannte ",high flyers“ oder "gazelle companies"). Hierbei weisen die FuE-intensiven Branchen tendenziell die bessere Wachstumsperformance auf. Unterschiedliche Wachstumsraten in den ersten Lebensjahren zwischen den Branchen resultieren auch aus branchenspezifischen mindestoptimalen Betriebsgrößen, die unterschiedlich schnell erreicht werden können.

Anhand von Daten des Hauptverbandes der Sozialversicherungsträger kann analysiert werden, inwieweit Betriebsgründungen (bzw. Schließungen) oder eher Beschäftigungsveränderungen im Unternehmensbestand die Entwicklung der Gesamtbeschäftigung determinieren. Zwischen 1994 und 1997 ist die Gesamtbeschäftigung im privaten Sektor Österreichs geringfügig um 0,9\% gewachsen (vergleiche Tabelle 6-3). Dabei resultieren 0,85 Prozentpunkte dieses Beschäftigungswachstums aus der Betriebsgründungdynamik (Saldo zwischen Marktaus- und Markteintritten), lediglich 0,05 Prozentpunkte aus Beschäftigungsveränderungen in bestehenden Betrieben. Insgesamt überwiegt also der Beschäftigungssaldo aus Marktein- und -austritten die Beschäftigungsveränderungen im Bestand. Diese Relation ist allerdings ausschließlich auf die Situation im produzierenden Gewerbe zurückzuführen, wo ein starker Beschäftigungsrückgang im Betriebsbestand zu verzeichnen war und dadurch auch die Gesamtveränderung nach unten "gedrückt“ wird. Der Dienstleistungssektor konnte im betrachteten Zeitraum deutliche Beschäftigungszunahmen verzeichnen, wobei die Zuwächse durch Veränderungen im Betriebsbestand höher waren als durch die Betriebsgründungsdynamik. Besonders avancierte Dienstleistungen (unternehmensnahe Dienstleistungen und hier vor allem F\&E-intensive Dienstleistungen) verzeichneten - trotz des doch relativ kurzen Zeitraumes - eine zweistellige Beschäftigungszunahme auf.

Tabelle 6-3: Veränderung des Beschäftigtenbestandes von 1995 bis 1997 nach Sektoren

\begin{tabular}{lccc}
\hline & $\begin{array}{c}\text { Gesamtveränderung } \\
\text { (in \%) }\end{array}$ & $\begin{array}{c}\text { durch Marktein- } \\
\text { und -austritte } \\
\text { (in \%) }\end{array}$ & $\begin{array}{c}\text { durch Veränderung im } \\
\text { Betriebsbestand } \\
\text { (in \%) }\end{array}$ \\
\hline Produzierender Sektor & $\mathbf{- 4 , 3 1}$ & $-\mathbf{0 , 5 2}$ & $\mathbf{- 3 , 7 9}$ \\
$\quad$ Verarbeitendes Gewerbe & $-5,33$ & $-0,64$ & $-4,69$ \\
$\quad$ davon FuE intensives VG & $-3,89$ & 0,34 & $-4,23$ \\
Bau & $-1,58$ & $-0,18$ & $-1,40$ \\
Distributionssektor & $\mathbf{1 , 2 3}$ & $\mathbf{0 , 3 9}$ & $\mathbf{0 , 8 4}$ \\
Handel & 2,40 & 0,46 & 1,94 \\
Dienstleistungen & $\mathbf{8 , 9}$ & $\mathbf{3 , 5 6}$ & $\mathbf{5 , 3 4}$ \\
Unternehmensnahe DL & 20,43 & 8,56 & 11,87 \\
$\quad$ davon FuE intensives DL & 40,82 & 17,27 & 23,55 \\
davon Beratung & 18,73 & 7,65 & $\mathbf{1 1 , 0 9}$ \\
Konsumbezogene DL & 4,74 & 1,75 & 2,99 \\
Gesamtbestand im privaten Sektor & $\mathbf{0 , 9 0}$ & $\mathbf{0 , 8 5}$ & $\mathbf{0 , 0 5}$ \\
\hline
\end{tabular}

Quelle: Hauptverband der österreichischen Sozialversicherungsträger, Berechnungen des ZEW Mannheim. 


\subsection{Das technologische Spezialisierungsprofil: Patentanmeldungen}

\section{Abbildung 6-3: Patent-Portfolio Österreichs am Europäischen Patentamt im internationalen Vergleich}

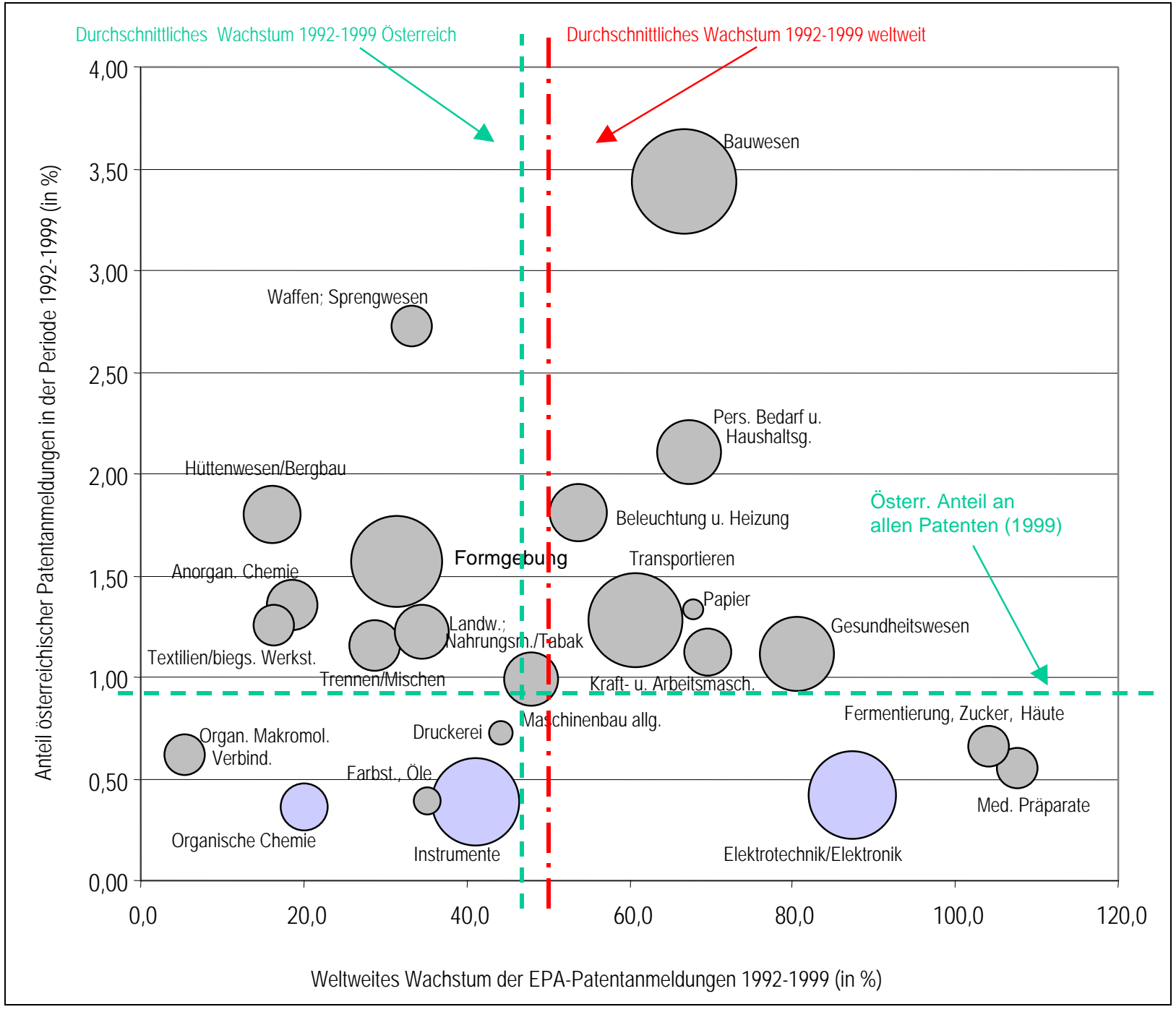

Quelle: Europäisches Patentamt; Berechnungen von tip

Anmerkung: Die Größe der Kreise bezieht sich auf die absolute Zahl der österreichischen Patentanmeldungen im Jahr 1999 (Summe = 794). Die Kreise mit Schachbrettmuster kennzeichnen jene drei Technologiefelder die weltweit die größte Bedeutung (in bezug auf absolute Zahl an Patentanmeldungen) aufweisen.

In Abbildung 6-3 werden die Patentanmeldungen Österreichs am Europäischen Patentamt (EPA) im Zuge der 90er Jahre international positioniert. Die Befunde lassen sich wie folgt zusammenfassen:

- Zwar liegt mit einem Wachstum zwischen 1992 und 1999 von 46 \% Österreich etwas unter dem generellen Anstieg der Patenmeldungen am EPA (Zunahme der Anmeldungen um $52 \%$ ), trotzdem lässt sich auch für Österreich im Zuge der 90er Jahre ein anhaltender Bedeutungsgewinn europäischer Patentanmeldungen feststellen. Durch die geringere Wachstumsrate verliert Österreich jedoch „Marktanteile“ am internationalen Markt für patentierbare Erfindungen.

- Die Struktur der Patentanmeldungen folgt dem bereits bekannten Muster (vergleiche die Technologieberichte 1997 und 1999): In Technologiefeldern, in denen weltweit die meisten europäischen Patente angemeldet werden (Instrumente; Elektrotechnik/Elektronik; Organische Chemie), ist 
Österreich vergleichsweise gering vertreten (d.h. der Anteil dieser Technologiefelder liegt unter dem generellen Anteil Österreichs von ca. 0,9\%).

- Gleichzeitig ist Österreich in Feldern mit rasch wachsender Bedeutung (überproportionale Zunahme der Patentanmeldungen) (Fermentierung; Zucker; Häute; Medizinische Präparate; Elektrotechnik/Elektronik) unterdurchschnittlich stark spezialisiert.

Insgesamt lassen sich somit anhand des Profils der Patentanmeldungen Schwächen der technologischen Spezialisierung Österreichs konstatieren. Dies korrespondiert mit den bekannten Defiziten der österreichischen Industriestruktur, nämlich der starken Ausrichtung auf traditionelle Branchen mit tendenziell niedrigem bis mittlerem Technologieniveau. 


\section{Informations- und Kommunikationstechnologien (IKT): die technologische Basis der wissensbasierten Ökonomie}

Die Erklärung wirtschaftlicher und gesellschaftlicher Transformationsprozesse im Kontext rapiden technologischen Wandels erfolgt in den 90er Jahren auf Basis teils konkurrierender, teils überlappender Konzepte wie z.B. Digital Economy, Informationsgesellschaft (Information Society), Learning Economy, Network Economy, New Economy und Wissensbasierte Ökonomie (Knowledge-based Economy). IKT bilden in zweierlei Hinsicht einen gemeinsamen Bezugspunkt für Erklärungsansätze wirtschaftlicher Transformationsprozesse in industrialisierten Ländern und für die Formulierung von nationalen Strategien zur Steigerung von Produktivität, Beschäftigung und Wachstum. Einerseits zeichnen sich Produkte und Dienstleistungen des IKT-Sektors durch hohe Wissensintensität - gemessen am erforderlichen Forschungsinput und der Qualifikation des Forschungspersonals - sowie eine überdurchschnittliche Dynamik bei Wachstum und Produktivität aus. Andererseits gelten IKT als Querschnittstechnologien, deren Einsatz in der Erstellung von Gütern und Dienstleistungen in allen Sektoren der Wirtschaft Transformationsprozesse auslöst.

Beginnend mit einer kurzen Darstellung des österreichischen Informationssektors konzentriert sich das vorliegende Kapitel deshalb in weiterer Folge auf die IKT-Diffusion in Österreich.

\subsection{Struktur und Entwicklung des österreichischen IKT-Sektors}

Abbildung 7-1: Beschäftigungsstruktur des österreichischen IKT-Sektors nach OECD-Definition, 1998

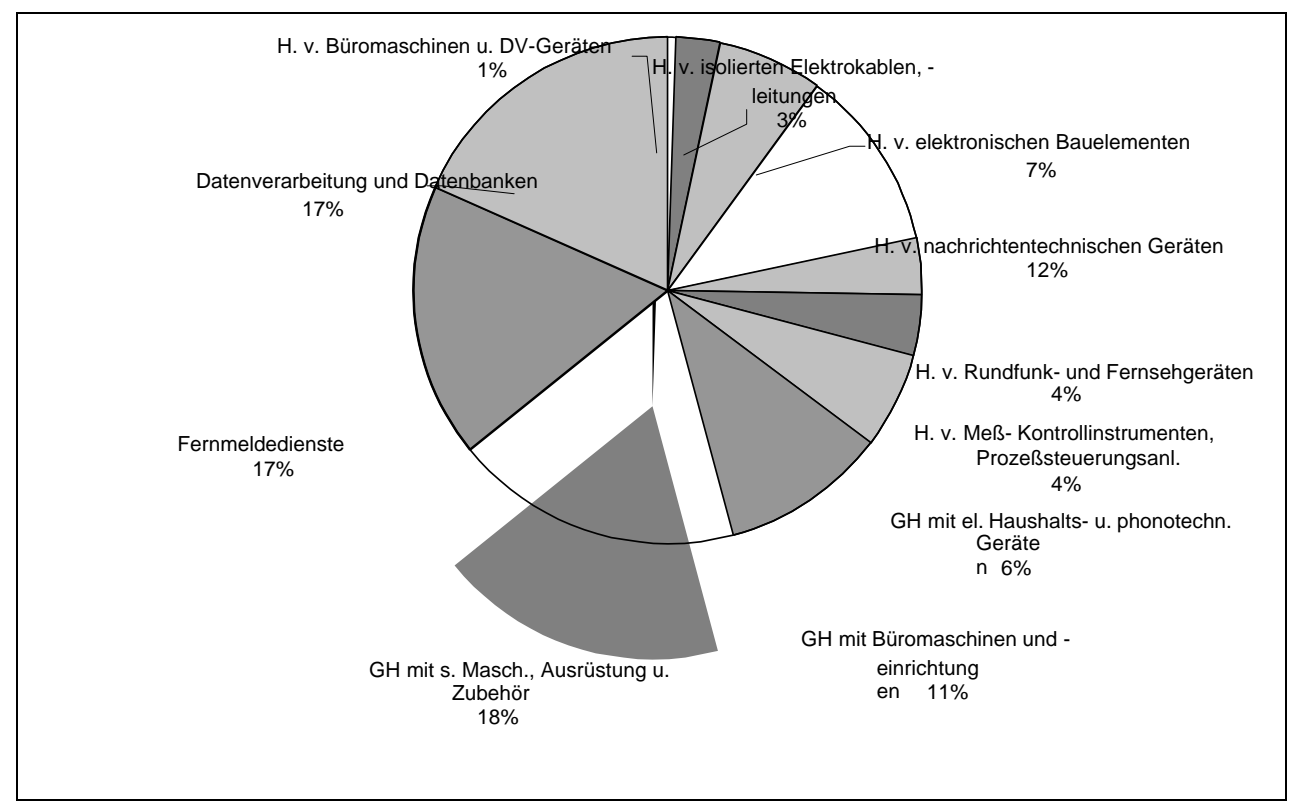

Quelle: Statistik Austria, Struktur- und Leistungserhebung 1998, Berechnungen von tip 


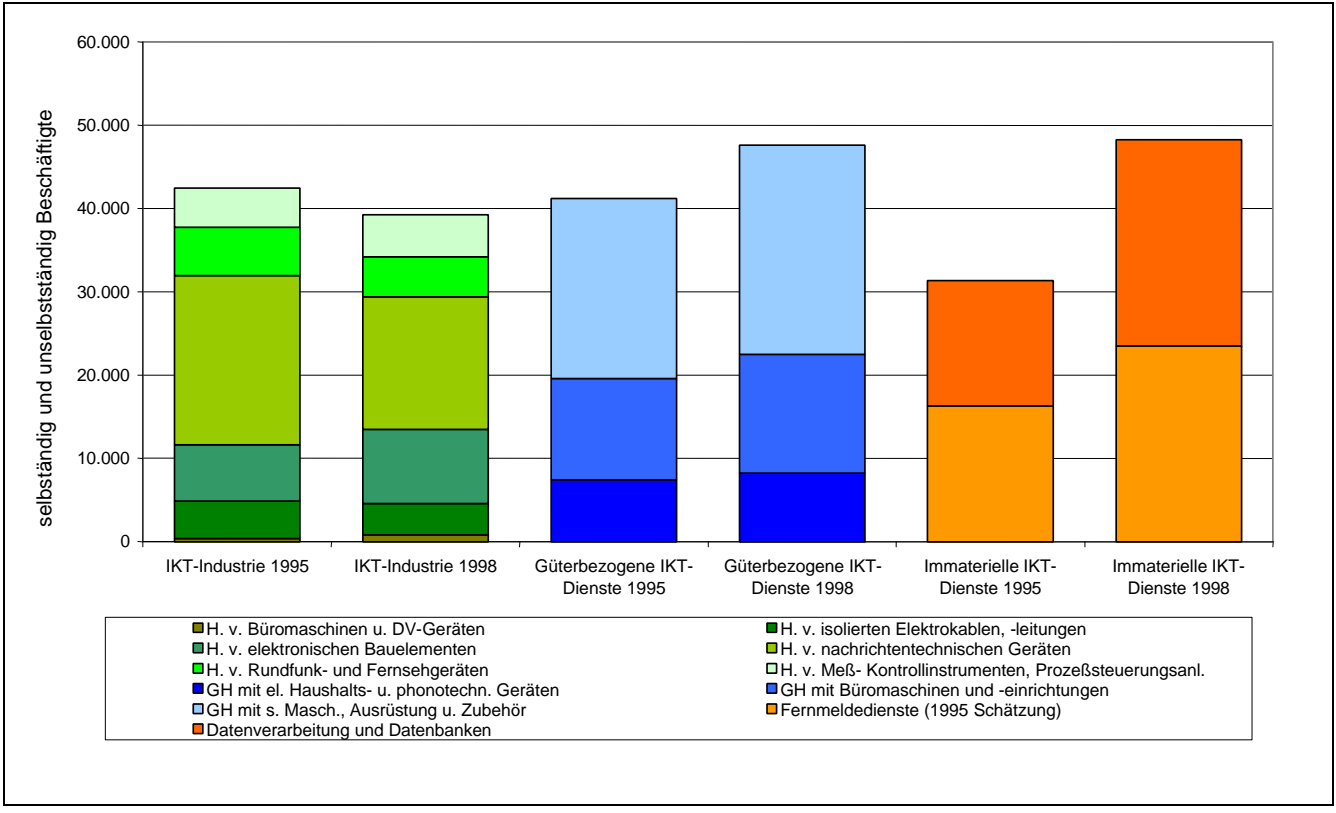

Quelle: Statistik Austria, Struktur- und Leistungserhebung 1995 und 1998, Berechnungen von tip

Der österreichische IKT-Sektor ${ }^{26}$ verzeichnete im Jahre 1998 in rund 11.000 Unternehmen mehr als 135.000 Beschäftigte. Mit einem Wertschöpfungsanteil von 8,4 \% bzw. einem Beschäftigtenanteil von $6 \%$ am gesamten Unternehmenssektor (ohne Landwirtschaft) hat der Sektor eine nicht unerhebliche Bedeutung für die österreichische Wirtschaft. Im Sektor sind im wesentlichen drei annähernd gleich große Gruppen von Produkten und Dienstleistungen zu unterscheiden (vergleiche Abbildung 7-1):

- Die IKT-Industrie (Beschäftigtenanteil $29 \%$ ) umfasst sowohl die Erzeuger von Bauteilen als auch von Telekommunikationsausrüstung und -endgeräten.

- Die güterbezogenen IKT-Dienstleistungen (Beschäftigtenanteil $35 \%$ ) beinhalten vorwiegend Großhandelsaktivitäten bzw. Handelsaktivitäten internationaler IKT-Unternehmen ohne nennenswerte Produktion in Österreich.

- Die besonders wachstumsstarken Branchen der Datenverarbeitung und der Telekommunikation (Fernmeldedienste) bilden gemeinsam die Gruppe der immateriellen IKT-Dienstleistungen (Beschäftigungsanteil $36 \%)$.

Insgesamt zeichnet sich der Sektor durch eine besonders hohe Dynamik aus. Das sektorale Wachstum zwischen 1995 und 1998 beispielsweise ist - gemessen an der Zahl der Unternehmen - mit $+48 \%$ und gemessen am Beschäftigtenstand mit $+17 \%$ überdurchschnittlich. Allerdings werden deutliche Unterschiede in den Teilsektoren erkennbar (vergleiche Abbildung 7-2):

- Zu einer erfreulich hohen Beschäftigungsausweitung kam es bei Datenverarbeitung und Telekommunikation ${ }^{27}$. Darüber hinaus weisen diese beiden Branchen mit einem Anstieg der Zahl der Unternehmen um jeweils ca. $90 \%$ eine außerordentlich hohe Gründungsdynamik auf.

${ }^{26}$ Die im folgenden verwendete Abgrenzung des IKT-Sektors entspricht der Definition der OECD. Vergleiche dazu OECD (2000c).

${ }^{27}$ Die Daten von Statistik Österreich geben erst ab 1998 den Umfang des Telekom-Sektors korrekt wieder, da bis dahin die Geschäftsbereiche Postdienste und Fernmeldedienste in der Post und Telekom Austria AG vereinigt waren und so der Beschäftigtenstand des Sektors Fermeldedienste, ÖNACE 64.20, auch die Mitarbeiter der heutigen Post AG und Postbus AG 
- Die heimische IKT-Industrie verzeichnete bei mäßigem Anstieg der Wertschöpfung eine deutliche Reduktion der Beschäftigtenzahl (-7\%) und lieferte somit im Vergleich mit den nordischen Staaten und den USA schwache Wachstumsimpulse für die Gesamtwirtschaft. Allerdings weisen Teile der IKT-Produktion (z.B. elektronische Bauteile) eine ähnlich hohe Wachstumsdynamik wie die immateriellen IKT-Dienste auf.

- Die expansive Entwicklung im IKT-Großhandel, dem auch die österreichischen Niederlassungen internationaler Technologiekonzerne angehören, spiegelt den steigenden Bedarf an IKT im privaten Bereich wie in der Wirtschaft ${ }^{28}$.

Insgesamt macht die Entwicklung im heimischen IKT-Sektor zwischen 1995 und 1998 den Strukturwandel deutlich erkennbar. Die Beschäftigungsentwicklung weist eine Verschiebung von der IKTProduktion hin zu IKT-Dienstleistungen auf. Unterschiede - und zwar sowohl in der Produktivitätsentwicklung als auch in der Handelbarkeit (Intensität des internationalen Wettbewerbs, Möglichkeiten für Produktionsverlagerungen) - spielen hierbei eine Rolle.

enthielt. Diese Beschäftigten, die 1995 nach Angaben dem Geschäftsbericht 1995 der PTA 37.412 der insgesamt 55.720 Mitarbeiter ausmachten, wurden vom Beschäftigtenstand der Fernmeldedienste abgezogen.

${ }^{28}$ Entwicklungen bei IKT-Industrie und IKT-Großhandel finden auch in der sektoralen Ausweitung des Handelsbilanzdefizits ihren Niederschlag. Siehe dazu auch OECD (2000c). 


\subsection{Entwicklung der IKT-Diffusion im internationalen Vergleich}

Infolge seiner hohen Wachstumsdynamik bei gleichzeitig rapidem technologischen Wandel liefert der IKT-Sektor einen direkten Beitrag für die Entwicklung hin zu einer wissensbasierten Ökonomie. Allerdings folgen aus der zunehmenden Nutzung von IKT für die Erstellung von Gütern und Dienstleistungen sowie für die Anbahnung und Abwicklung kommerzieller Transaktionen in anderen Sektoren (vergleiche dazu weiter unten die Box zur Verbreitung von CIM-Technologien in Österreich) mindestens ebenso bedeutsame Effekte.

Produkte und Anwendungen wie Computer und Internet gelten mittlerweile als selbstverständliche Werkzeuge. IKT erfüllen die wesentlichen Eigenschaften von generischen Technologien bzw. Querschnittstechnologien (general-purpose technologies). Die zunehmende IT-Intensität - gemessen beispielsweise an den IT-Ausgaben in Prozent des Bruttoinlandsprodukts - ist ein allen entwickelten Industrieländern gemeinsames Phänomen und kann als Indiz für die zunehmende Diffusion von IKT gewertet werden. Während der 90er Jahre ist für diesen Indikator ein starker Anstieg zu beobachten, und auch in fortgeschrittenen Ländern wie z.B. den USA ist bislang keine Sättigung erkennbar (vergleiche Abbildung 7-3). Der internationale Vergleich zeigt außerdem, dass ein Aufholen Europas gegenüber den USA zumindest bis Ende der 90er Jahre nicht gelungen ist und die Situation innerhalb der Europäischen Union nach wie vor durch starke Disparitäten (regionales Nord-Süd-Gefälle) gekennzeichnet ist.

Im Länderranking hat sich die Position Österreichs im hinteren europäischen Mittelfeld kaum verändert. Die IT-Intensität ist hierzulande noch immer niedriger als in der Spitzengruppe, bestehend aus den nordischen Ländern, der Schweiz und den Niederlanden. Seit Mitte der 90er Jahre besteht zudem ein annähernd gleicher (geringer) Rückstand zum Durchschnitt der Europäischen Union. Trotz eingeschränkter Aussagekraft dieses aggregierten Indikators zur Diffusion von IT-Anwendungen im Unternehmensbereich ist davon auszugehen, dass ein genereller Aufholprozess bislang noch weitgehend ausgeblieben ist.

Ein konkretes Beispiel für zögerliche Aufholprozesse Österreichs bei der IT-Diffusion in den 90er Jahren liefert die Entwicklung der PC-Dichte (vergleiche Abbildung 7-4). Im Vergleich von 24 überwiegend westeuropäischen OECD-Ländern führt Österreich im Jahre 1999 das untere Drittel der untersuchten Länder mit 256,8 PCs pro 1.000 Einwohner an. Österreich liegt damit hinter vielen EU-Mitgliedern zurück und nur unwesentlich über dem Vergleichswert für die gesamte Europäische Union $(248,6)$. Allerdings soll darauf hingewiesen werden, dass bis 1994 Österreich unter dem EU-Durchschnitt lag und erst im Jahr 1995 eine im Vergleich zum EU-Schnitt höhere PC-Dichte aufwies. Die Ausgangssituation Anfang der 90er Jahre (bzw. in den 80er Jahren) war also durchaus jene eines "Nachzüglers“. Insofern ist das, wenn auch verhaltene, Aufholen bzw. Anschließen an den „Durchschnitt“ bemerkenswert. 


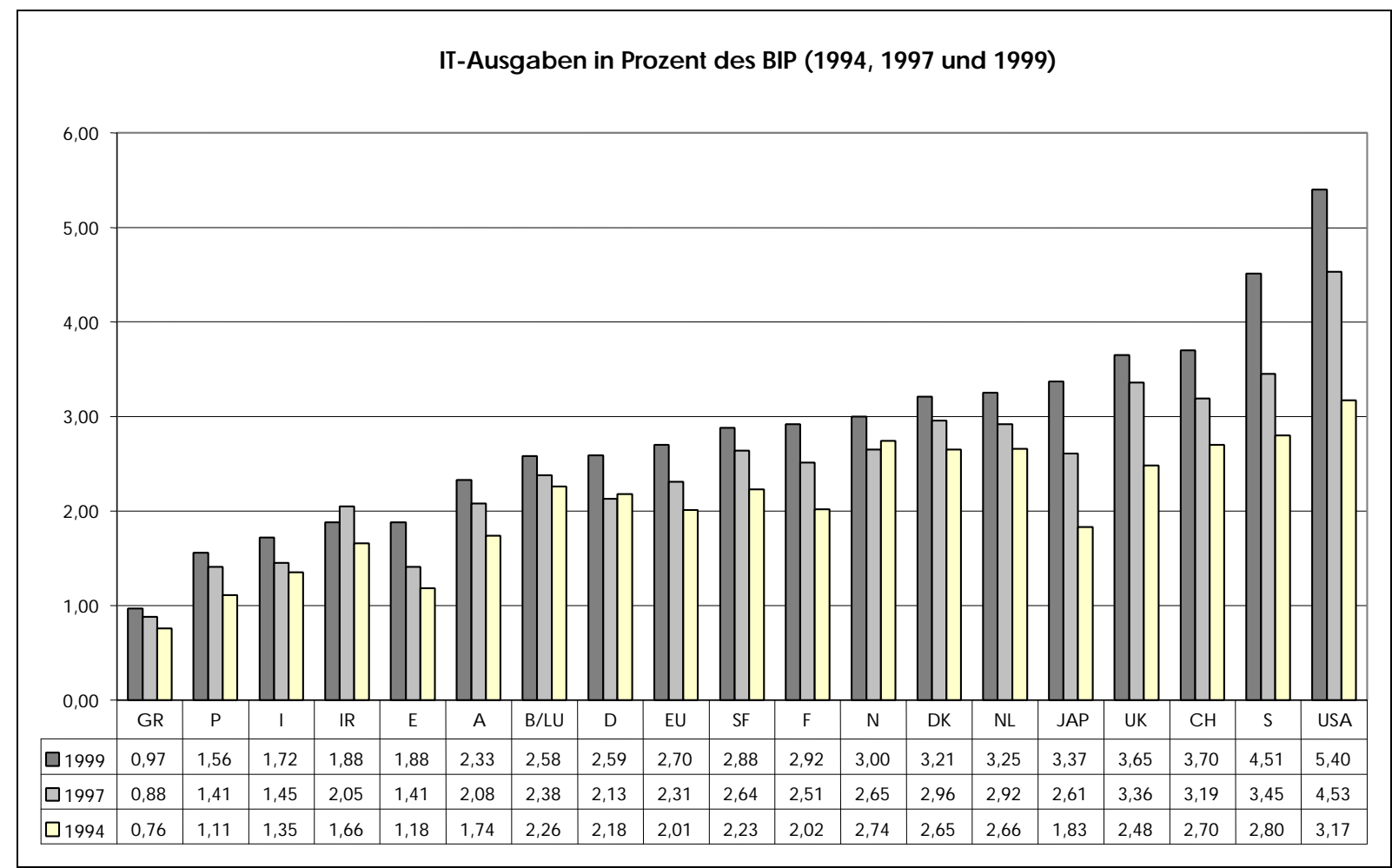

Quelle: EITO.

Aus der Wachstumsdynamik im Zeitraum von 1991 bis 1999 lässt sich kein Aufholen erkennen. So ist der - in logarithmischer Skalierung - fast parallel erscheinende zeitliche Verlauf für die Ländergruppen ein Indiz für das Ausbleiben von Aufholprozessen. Dasselbe Ergebnis lässt sich aus dem direkten Vergleich der Wachstumsraten ableiten (vergleiche Abbildung 7-4). Gemessen an der durchschnittlichen jährlichen Wachstumsrate (CAGR 1992-1999) liegt Österreich mit einem Wert von 16,3\% über den Vergleichswerten der Europäischen Union (15,1\%) und des mittleren Drittels (15,2\%). Allerdings weisen sowohl das untere (16,9\%) als auch das obere Terzil $(17,1 \%)$ höhere durchschnittliche Wachstumsraten auf. Der Rückstand Österreichs gegenüber der Spitzengruppe beträgt somit mehrere Jahre, und ein Aufschließen an diese Vorreiter ist bei gegebenen Wachstumsraten zumindest kurzfristig nur schwer möglich. 
PCs pro 1000 Einwohner (1991-1999)

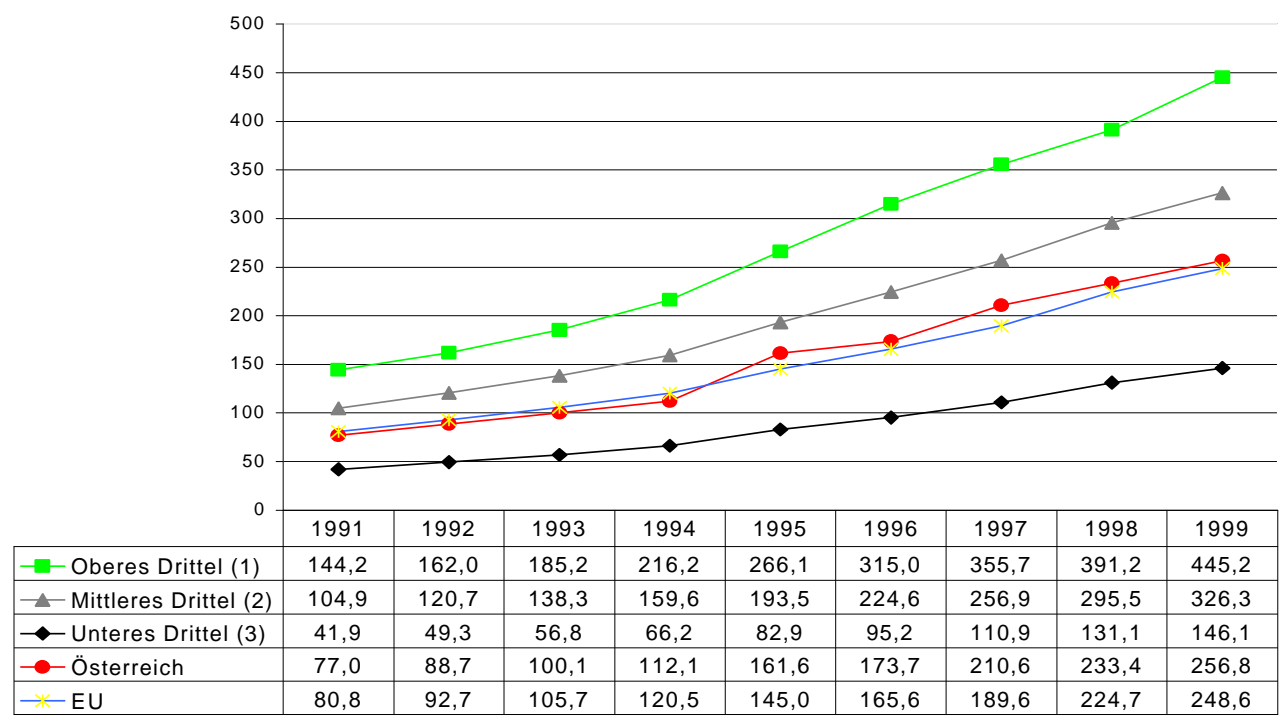

PCs pro 1000 Einwohner (1991-1999; logarithmische Skalierung)

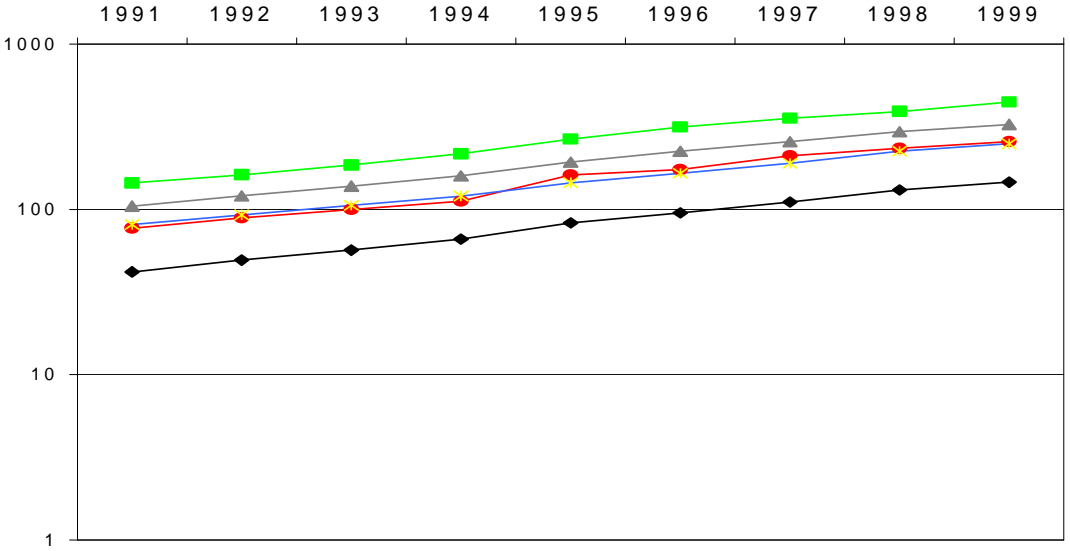

(1).. Mittelwert für Australien, Dänemark, Irland, Luxembourg (ab 1996), Norwegen, Schweden, Schweiz und USA.

(2).. Mittelwert für Belgien, Deutschland, Finnland, Großbritannien, Japan, Kanada, Neuseeland und die Niederlande.

(3).. Mittelwert für Frankreich, Griechenland, Italien, Korea, Mexiko, Österreich, Portugal und Spanien.

Quelle: ITU, WIFO-Berechnungen für 24 ausgewählte OECD-Staaten. 
Dass die vergleichsweise niedrige PC-Dichte in Österreich nicht allein auf eine Schwäche bei privater Nutzung zurückzuführen ist, sondern auch mit niedriger Nutzungsintensität in der betrieblichen Anwendung zusammenhängt, zeigt eine europäische Umfrage ${ }^{29}$. Aus dieser Befragung folgt, dass nur 42,5\% der heimischen Arbeitnehmer einen Computer für die Arbeit nutzen. Österreich liegt knapp unterhalb des Vergleichswerts für die gesamte Europäische Union (45\%) und hinter Deutschland (46,0 \%) aber deutlich hinter den nordischen Ländern (Schweden: 74,3\%; Dänemark: 67,3\%; Finnland: $56,9 \%)$ und den Niederlanden (67,5\%). Damit bestätigt sich der für die PC-Dichte vorgelegte Befund auch in einer niedrigen Nutzung von PCs für berufliche Zwecke. Diese Ergebnisse sind jedoch vor dem Hintergrund der jeweiligen Wirtschaftsstrukturen zu sehen. Österreich weist, wie auch Deutschland, insbesondere im Vergleich zu den nordischen Ländern (v.a. Schweden und Dänemark) noch einen hohen Anteil des sekundären Sektors auf. Da die PC-Nutzung in vielen Berufen des Dienstleistungssektors allgemein höher ist als in der produzierenden Industrie, ergibt sich somit ceteris paribus - zwangsläufig eine geringere Nutzungsdichte. Insbesondere unternehmensnahe Dienstleistungen, die eine besonders hohe PC-Nutzungsdichte aufweisen, sind in Österreich im Vergleich zu Ländern wie Schweden oder der Schweiz noch immer unterdurchschnittlich vertreten.

Trotz eines Diffusions-Rückstandes gegenüber Ländern der Spitzengruppe, der anhand von Indikatoren wie z.B. IT-Intensität und PC-Dichte nachweisbar ist, sind die Hinweise einer Entwicklung der österreichischen Volkswirtschaft hin zu einer wissensbasierten Ökonomie unverkennbar. Ein Indiz für strukturelle Änderungen liefert beispielsweise die Zunahme der Softwareinvestitionen in der Wirtschaft. Software gilt als eine Form von kodifiziertem Wissen, die einerseits unter Nutzung hochqualifizierten Humankapitals erstellt wird. Andererseits ist der Grad ihres Einsatzes als universelles Instrument zur Erfassung, Verarbeitung und Speicherung von Information auch ein Indiz von der Informationsintensität von Branchen oder Unternehmen.

Das Wachstum der Softwareinvestitionen in Österreich ist beachtlich. Aus Erhebungen der Volkswirtschaftlichen Gesamtrechnung (Statistik Austria) folgt, dass sich die Investitionen in Software zwischen 1988 und 1999 mit einem Anstieg von 4,2 Mrd. auf 13,2 Mrd. ATS in etwa verdreifacht haben. Softwareinvestitionen zählen damit zu den am raschesten wachsenden Bestandteilen des Kapitalstocks.

Bei genauerer Betrachtung der Softwareinvestitionen fallen im Branchenvergleich große Disparitäten sowohl in bezug auf die Softwareintensität pro Mitarbeiter als auch den Softwareanteil an den Bruttoinvestitionen auf. Insgesamt entfällt auf den Unternehmenssektor mit Softwareinvestitionen von rund 8,8 Mrd. ATS der Hauptteil der Investitionstätigkeit (vergleiche Statistik Austria 2000a, 2000b). Softwareinvestitionen spielen in allen Branchen eine bedeutende Rolle. Allerdings ist ähnlich wie bei Forschungs- und Entwicklungsausgaben eine Ungleichverteilung zwischen den Wirtschaftsklassen - insbesondere eine Konzentration auf den Dienstleistungssektor - unverkennbar (während bei den F\&EAufwendungen die Industrie dominiert). So übertreffen beispielsweise die Softwareausgaben des Bankensektors mit 2,054 Mrd. ATS die Softwareinvestitionen der gesamten Sachgüterproduktion $(1,66 \mathrm{Mrd} \text {. ATS })^{30}$. Banken und Versicherungen, Datenverarbeitung, Telekommunikation und unternehmensnahe Dienstleistungen stellen - neben der Gruppe forschungsintensiver Industriezweige den zweiten Kernbereich einer wissensbasierten Ökonomie dar. Bei einem Beschäftigungsanteil von $17 \%$ leisten sie $56 \%$ der gesamten Softwareinvestitionen im Unternehmenssektor Österreichs.

\footnotetext{
${ }^{29}$ Eurobarometer, November 2000

${ }^{30}$ Werte für 1998.
} 
Abbildung 7-5: Struktur der Softwareinvestitionen des österreichischen Unternehmenssektors 1998

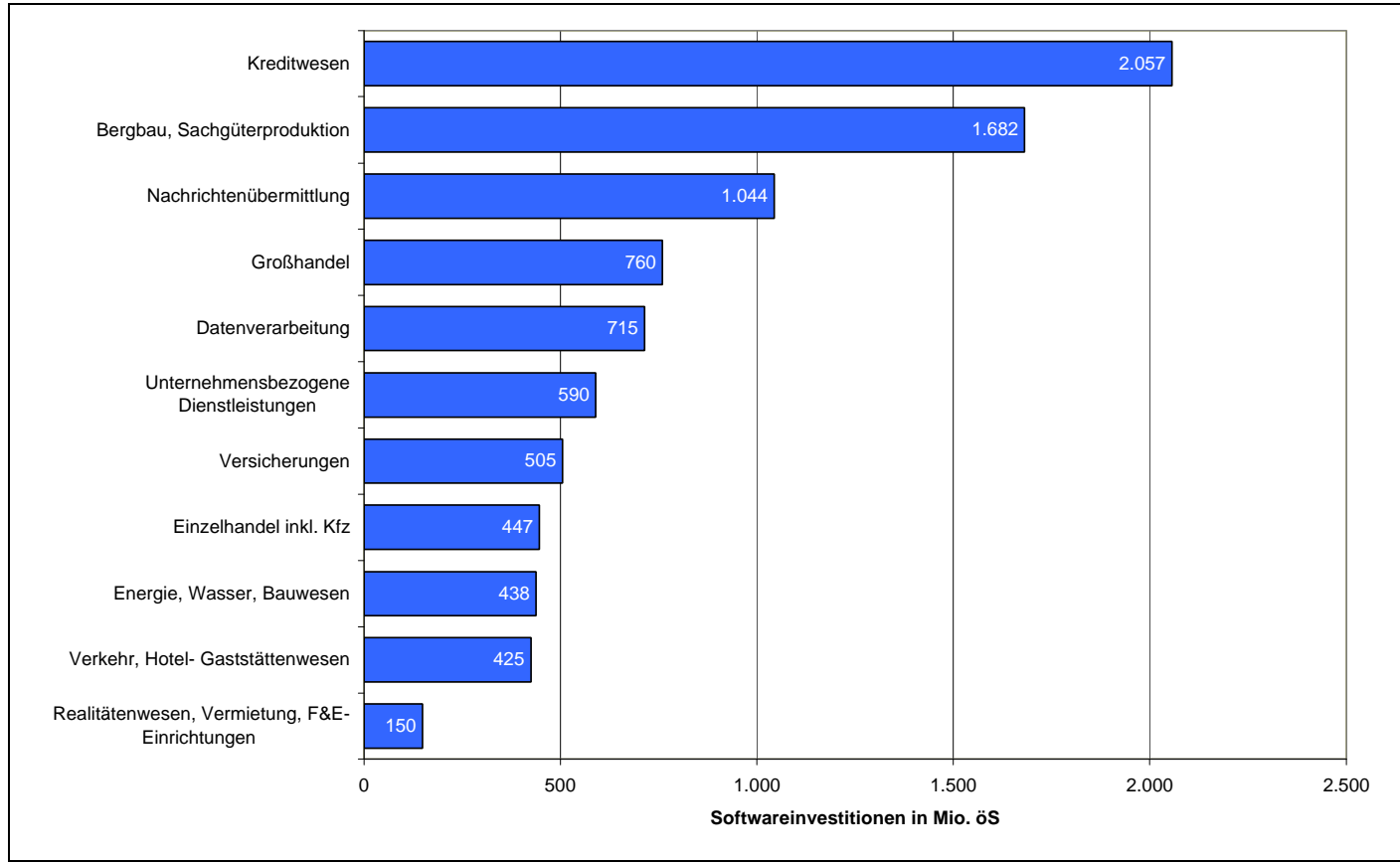

Quelle: Statistik Austria; Struktur- und Leistungserhebung 1998.

Abbildung 7-6: Softwareintensität des österreichischen Unternehmenssektors, 1998

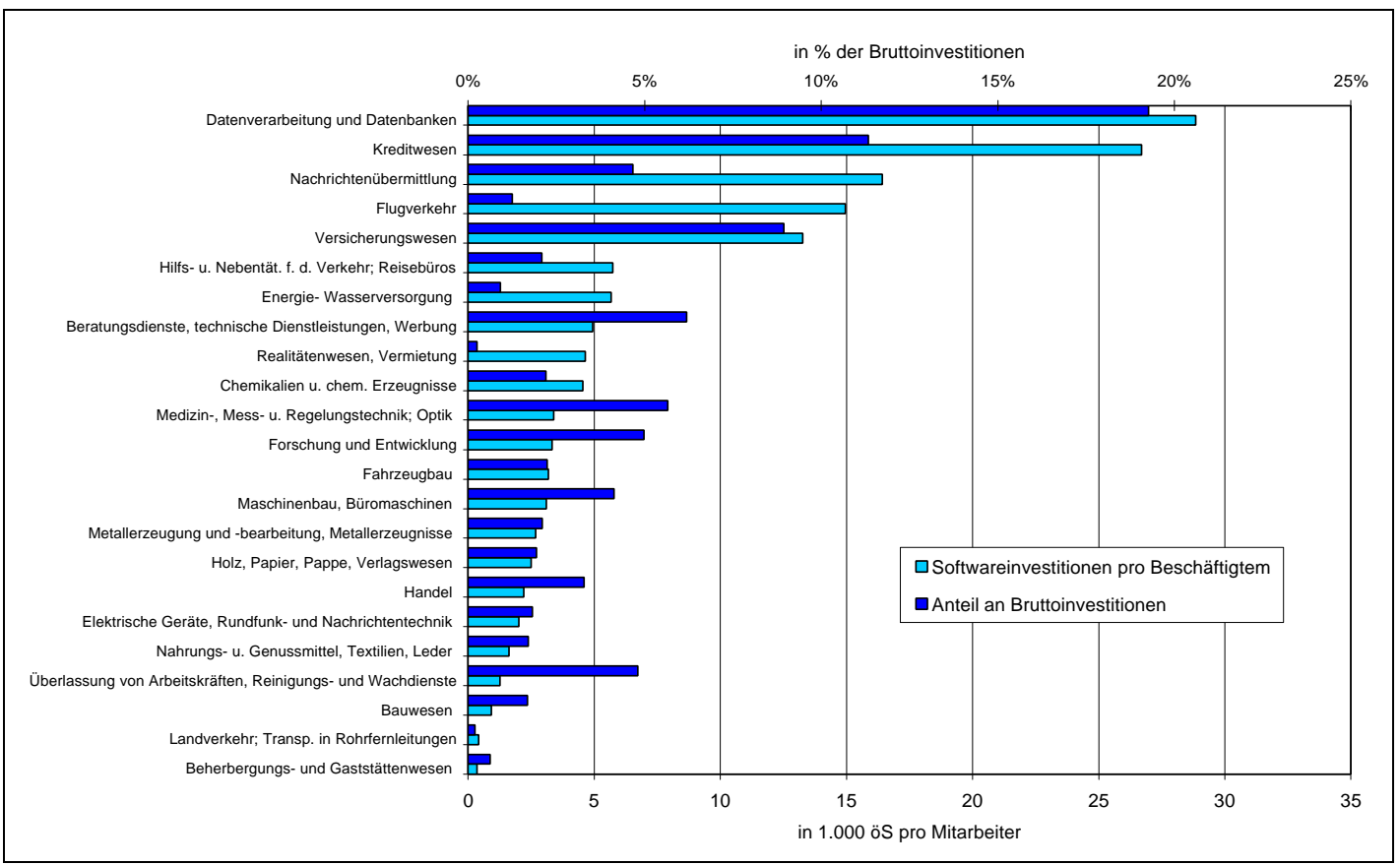

Quelle: Statistik Austria; Struktur- und Leistungserhebung 1998.

Disparitäten in der Softwareintensität bzw. die starke Position einiger besonders wissensintensiver Dienstleistungsbranchen lassen sich vorwiegend durch Anwendungsmöglichkeiten und bestehende Nutzungsformen (Front- und Back-office-Anwendungen, Kundendatenbanken etc.) erklären. Darüber hinaus ist Software vielfach in andere Investitionsgüter (z.B. Maschinen und Anlagen) inkorporiert und scheint nicht explizit als Investition auf. Gleichzeitig wird erkennbar, dass Softwareinvestitionen hin- 
sichtlich der Modernisierung von Prozessen (Prozessinnovationen) im Dienstleistungsbereich einen mit Anlageinvestitionen in der Sachgüterproduktion vergleichbaren Stellenwert haben.

Der IKT-Sektor reicht über IT im engeren Sinn hinaus und beinhaltet neben Kommunikationsausrüstungen und -geräten auch Telekommunikationsdienste. Letztere bilden einen wichtigen infrastrukturellen Baustein der wissensbasierten Ökonomie. Ein Abgehen vom monopolistischen Angebot sowie die Beschleunigung des technologischen Wandels (Digitalisierung, Konvergenz etc.) haben im Telekomsektor während der 90er Jahre zu einer Beschleunigung des Strukturwandels geführt. Die Diffusion von Telekomdiensten hat - anders als im IT-Bereich - durch die Liberalisierung des österreichischen Marktes infolge von Preissenkungen, der beschleunigten Modernisierung der Netzinfrastruktur und des Angebots neuer Dienstleistungen starke Impulse erhalten. Beispielsweise gehört Österreich in der Mobilkommunikation in Hinblick auf niedrige Kosten und hohe Marktdurchdringung nunmehr zu den führenden Ländern Europas. Damit sind auch die ersten Schritte zum Aufbau einer universellen, mobilen Infrastruktur gesetzt. Dies ist umso mehr bemerkenswert als Österreich aus einer eher ungünstigen Ausgangssituation gestartet ist (verspätete Liberalisierung, anfangs niedrige Nutzungsdichten und hohe Preise).

Die Diffusion von Telekom-Anwendungen wird sowohl unter gesellschafts- als auch unter wirtschaftspolitischen Gesichtspunkten als strategisch wichtig eingeschätzt. Hohe Erwartungen richten sich an das Internet, die Brücke zwischen IT-Welt und Telekommunikation, eine Querschnittstechnologie mit innovativen Impulsen für geschäftliche Anwendungsumgebungen. Das Internet fungiert zudem als technische Plattform für Anwendungen des eCommerce. Letztlich hat eine Ausweitung digitaler Kommunikation Implikationen, die von erforderlichen individuellen Fähigkeiten über Re-Organisationsbedarf in Unternehmen bis hin zu Verschiebungen der Kräfteverhältnisse zwischen Marktteilnehmern reichen.

Im Vergleich mit der PC-Dichte lassen sich für Österreich in der Nutzung des Internet deutlich positive Entwicklungen ausmachen. Gemessen an der Zahl der Internetnutzer pro 1000 Einwohnern liegt Österreich im mittleren Terzil von 24 ausgewählten OECD-Ländern (vergleiche Abbildung 7-7). Österreich liegt deutlich über dem Durchschnitt der gesamten EU und hat - rein zeitlich gesehen - einen Rückstand zur führenden Ländergruppe von lediglich rund einem Jahr. Die Wachstumsdynamik des Indikators weist auf Aufholprozesse zwischen den Ländergruppen hin. So zeigt sich beispielsweise in Abbildung 7-7(bei logarithmischer Skalierung) eine leichte Konvergenz über den zeitlichen Verlauf. In dieselbe Richtung weisen die durchschnittlichen Wachstumsraten der 90er Jahre (CAGR 92-99), die Ländern mit niedrigem Ausgangsniveau in der überwiegenden Mehrzahl stärkere Wachstumsraten bescheinigen.

Aus der Internetnutzerdichte lässt sich keine stringente Einschätzung der Positionierung Österreichs hinsichtlich der Nutzung elektronischer Medien für die geschäftliche Kommunikation treffen. Messprobleme und fehlende quantitative Untersuchungen mit ausreichendem Repräsentationsgrad verhindern eine fundierte Bewertung. Ein - auch von der OECD verwendeter - Indikator, um das Ausmaß kommerzieller Anwendungen im Internet abschätzen zu können, ist die Dichte der „secure web servers" (Anzahl pro Million EinwohnerInnen), die laufend von diversen privaten Internet research organisations (z.B. Netcraft) ermittelt wird. Hier liegt Österreich (1999 basierend auf Angaben von Netcraft) im oberen europäischen Mittelfeld in etwa gleichauf mit Norwegen und Großbritannien (die Schweiz, Schweden und Finnland stellen die Spitzengruppe). Nichteuropäische englischsprachige Länder (USA, Neuseeland und Kanada) weisen allerdings durchwegs eine deutlich höhere Dichte als die europäischen Länder. 


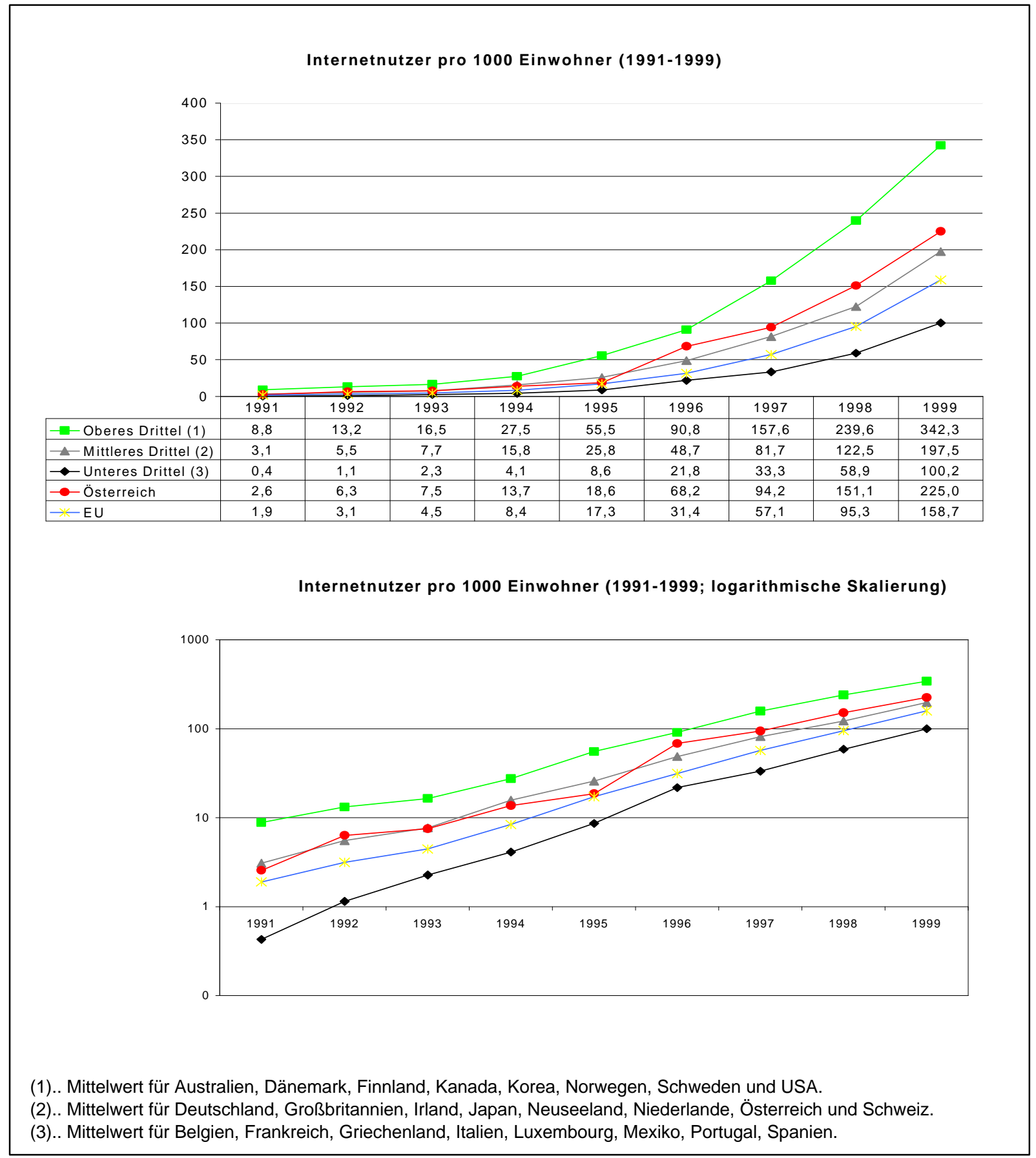

Quelle: ITU, WIFO-Berechnungen für 24 ausgewählte OECD-Staaten. 


\section{Verbreitung, Einsatz und Entwicklungstrends von CIM-Technologien in der österreichischen Sachgüterproduktion}

\section{Zeitprofil der CIM-Anwendung}

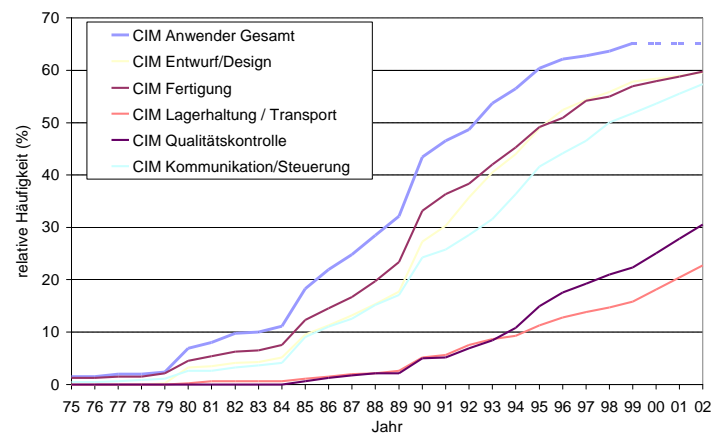

CIM-Anwendung im Jahr 1999 nach Betriebsgröße ${ }^{31}$

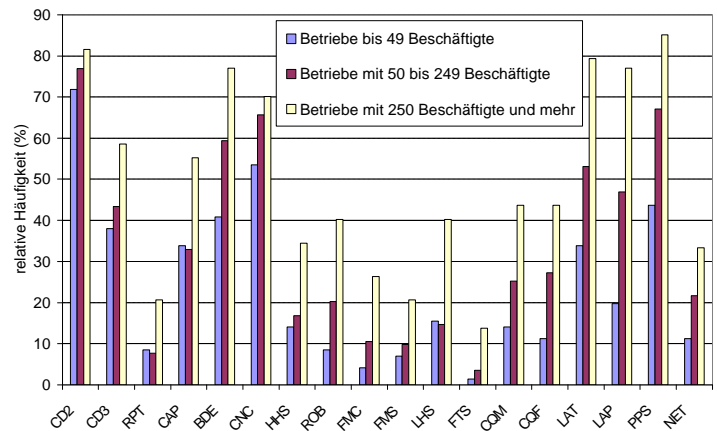

Quelle: FlexCIM-Erhebung 1999, Berechnungen ARCS

Die Verbreitung von fortgeschrittenen Fertigungstechnologien ist ein wichtiger Indikator für die Fähigkeit einer Ökonomie zu Prozessinnovationen. Die österreichische Sachgüterproduktion ist traditionell stärker als die in anderen Ländern auf Prozessinnovationen orientiert. Gleichwohl war in den fortgeschrittensten Fertigungstechnologien wie z.B. in der Computer-Integrierten Fertigung (CIM) in den 80er Jahren ein Rückstand festzustellen.

In den 90er Jahren setzte dagegen ein rasches Wachstum ein. 1999 setzten bereits ca. $65 \%$ der Betriebe der österreichischen Sachgüterproduktion (ÖNACE-Abschnitte 10 bis 36) mit mehr als 20 Beschäftigten computerintegrierte Fertigungsverfahren (CIM) ein (Geyer et al. 2000). Am häufigsten erfolgt der CIM-Einsatz für Entwurf und Planung (vor allem CAD-Anwendungen) sowie in der Fertigung (häufigste Technologien: CNC und Betriebsdatenerfassung). In diesen beiden Anwendungsbereichen ist bereits ein Abflachen der Diffusionskurve festzustellen.

Weiterhin zunehmen werden dagegen neuere CIM-Technologien für Produktionskommunikation und steuerung (PPS, interne und externe Netzwerke), wobei vor allem die Verbreitung von Netzwerken mit Kunden und/oder Lieferanten in den kommenden Jahren stark wachsen wird. Eine relativ geringe Verbreitung von CIM besteht nach wie vor in den Bereichen Qualitätskontrolle und Handling (Lagerhaltungssysteme, Transportsysteme), wobei letztere CIM-Technologien aber auch nur in einem Teil der Betriebe sinnvoll einsetzbar sind.

Die Verbreitung bzw. die Intensität des Einsatzes von CIM in der österreichischen Sachgüterproduktion ist deutlich von der Betriebsgröße abhängig. Während bei "alten" CIM-Technologien (z.B. zweidimensionales CAD, CNC) die Unterschiede in der Verbreitung zwischen großen und kleinen Betrieben vergleichsweise gering ausfallen, sind diese avancierteren CIM-Anwendungen (z.B. RPT, FMC, FMS) besonders deutlich ausgeprägt.

Im Jahr 1998 betrug der Anteil der in der FlexCIM-Erhebung 1999 erfassten Betriebe, die mehr als 80 $\%$ des Umsatzes mit CIM-gefertigten Produkten erwirtschafteten, rund $17 \%$. Dieser Anteil wird bis zum Jahr 2002 rasch wachsen und auf $32 \%$ ansteigen (Geyer et al. 2000).

${ }^{31}$ BDE: Betriebsdatenerfassung; CAP: computerunterstützte Arbeitsvorbereitung; CD2: CAE/CAD zweidimensional; CD3: CAE/CAD dreidimensional; CNC: CNC/DNC-Maschinen inklusive Laser; CQF: Qualitätskontrolle Fertigprodukte; CQM: Qualitätskontrolle Material / Ware in Arbeit; FMC: Flexible Produktionszellen; FMS: Flexible Produktionssysteme; FTS: Transportsysteme; HHS: Handhabungssysteme (mit drei und weniger Freiheitsgraden); LAP: LAN in der Produktion; LAT: LAN für technische Daten; LHS: Lagerhaltungssysteme; NET: Netzwerke mit Lieferanten oder Kunden; PPS: Produktionsplanungsund -steuerung; ROB: Industrieroboter; RPT: Rapid Prototyping / Simulation 


\subsection{Schlussfolgerungen}

Die Wachstumserwartungen einer durch einen starken IKT-Sektor geprägten "New Economy“ haben sich in Österreich, wie auch in den meisten europäischen Ländern, bislang nur zum Teil erfüllt. Gleichzeitig zeigen sich in bezug auf die Diffusion und Nutzung von IKT deutliche Defizite gegenüber den führenden Ländern. Da die führenden Länder bei der Anwendung von IKT auch gleichzeitig die höchsten Wachstumsraten bei IKT-Ausgaben aufweisen, ist im internationalen Vergleich kein durchgängiger Trend zu Konvergenz feststellbar. Ein kurzfristiges, gleichsam "automatisches" Aufschließen Österreichs an die Spitzengruppe ist deshalb nicht zu erwarten. Die empirische Evidenz ist uneinheitlich; partiellen Aufholprozessen (wie z.B. in der Mobiltelefonie oder abgeschwächt in der Internetdichte) steht ein gleichbleibender Abstand in anderen Bereichen (z.B. IT-Investitionen) gegenüber. Insgesamt lässt sich feststellen, dass technologiepolitische Initiativen im weiteren Sinne notwendig erscheinen.

Der technologische Wandel löst in zweierlei Hinsicht Handlungsbedarf aus: Einerseits leistet der IKTSektor direkt und indirekt einen wesentlichen Beitrag für Wachstum und zur Erhaltung der Wettbewerbsfähigkeit (von der Unternehmensebene bis zur gesamtwirtschaftlichen Ebene) und sollte daher in seinem Wachstum unterstützt werden. Andererseits gilt es aber auch, eine neue gesellschaftliche Segmentierung (Digital Divide), die aus unterschiedlichen Möglichkeiten im Zugang zu und der Nutzung von IKT resultieren, zu vermeiden.

Sowohl die Stärkung der internationalen Wettbewerbsfähigkeit als auch die Vermeidung einer Digital Divide bilden die wesentlichen Grundlinien der von der Europäischen Kommission auf den Gipfel von Lissabon formulierten Initiative „eEurope ${ }^{\text {“32 }}$. Zentrale Maßnahmen betreffen die Sicherstellung einer leistungsfähigen, für alle Bevölkerungsgruppen zugänglichen Telekommunikationsinfrastruktur, Qualifikation im Umgang mit Internet und Neuen Medien sowie die Förderung der Verbreitung von ecommerce und anderen Anwendungen des Internets in verschiedenen Bereichen unter Einschluss des öffentlichen Sektors.

Die Initiative eEurope hat allerdings weder hinsichtlich Art noch im Hinblick auf ihren Umfang den Anspruch, eine dem Querschnittscharakter von IKT entsprechende nationale Technologiepolitik zu ersetzen. Mitglieder der Union, die auf dem Weg in die Informationsgesellschaft besonders weit fortgeschritten sind, haben in den vergangenen Jahren vielfach an der Entwicklung und Umsetzung von IKT-bezogenen, technologiepolitischen Strategien gearbeitet; in Teilbereichen gehen die Länderinitiativen wesentlich über die Initiative „eEurope“ hinaus (siehe nachfolgende Box).

Auch in Österreich hat die Kommissionsinitiative eEurope zu einer spürbaren Intensivierung der Aktivitäten zur Informationsgesellschaft beigetragen. Aufgrund des Querschnittscharakters von IKT sind eine Reihe von Ministerien involviert:

- Das Bundesministerium für Bildung, Wissenschaft und Kultur fördert im Rahmen seiner IKTStrategie $^{33}$ acht Programmschwerpunkte, wobei Schwerpunkte auf die IKT-Ausstattung von Schulen,, die Verbesserung der Netzinfrastruktur von Universitäten und anderen Bildungseinrichtungen sowie die Adaptierung der Ausbildungsinhalte und Verbesserung der Ausbildungsmöglichkeiten ausgerichtet sind.

- Das Bundesministerium für Verkehr, Innovation und Technologie fördert Forschung mit IKT-Bezug in insgesamt sechs K-plus-Kompetenzzentren sowie Initativen im Verkehrsbereich (z. B. Logistik).

\footnotetext{
${ }^{32}$ Siehe Europäische Kommission (2000).

${ }^{33} \mathrm{vgl}$. http://www.bmbwk.gv.at/ikt-strategie/grundl.htm
} 
Auch der Regulator für den Telekommarkt, dem bei der Senkung der Zugangskosten zur Telekom-Infrastruktur eine entscheidende Rolle zukommt, ist beim BMVIT angesiedelt.

- Das Bundesministerium für Wirtschaft und Arbeit hat in sieben Arbeitsgruppen ${ }^{34}$ Maßnahmenvorschläge zu verschiedenen Aspekten von „e-business“ ausarbeiten lassen, die im Laufe des Jahres umgesetzt werden. Im Rahmen des $\mathrm{K}^{\text {ind }} / \mathrm{K}^{\text {net }}$-Projekts werden drei Kompetenzzentren im Bereich e-business gefördert.

- Das Bundesministerium für öffentliche Leistung und Sport befasst sich als IT-Koordinator des Bundes mit Anwendungsmöglichkeiten von IKT in der öffentlichen Verwaltung (e-government).

- Das Bundesministerium für Justiz adaptiert rechtliche Bestimmungen in verschiedenen Bereichen (etwa durch die Umsetzung der EU-Richtlinie über den elektronischen Geschäftsverkehr) an die Erfordernisse neuer Kommunikationsmittel.

- Den Ministerien nachgelagerte Fonds (etwa FWF, FFF, ERP) fördern im Rahmen ihrer allgemeinen Tätigkeit sowie in Sonderprogrammen Forschungs-, Entwicklungs- sowie Investitionsprojekte mit IKT-Bezug.

Eine gemeinsame Strategie der Bundesregierung, die die einzelnen Programme der Ministerien zu einer Gesamtstrategie vereint, befindet sich in Ausarbeitung. Ein entsprechendes Dokument des Bundesministeriums für öffentliche Leistungen und Sport, das den 1996 vorgestellten Aktionsplan zur Informationsgesellschaft der Bundesregierung fortsetzt, wird 2001 fertiggestellt.

\footnotetext{
${ }^{34} \mathrm{vgl.}$ http://www.bmwa.gv.at/ebusiness/index.htm
} 


\section{Internationale Initiativen für die Informationsgesellschaft ${ }^{35}$}

Nationale Strategien fortgeschrittener Länder wie z.B. Dänemarks, Deutschlands, Großbritanniens und der Niederlande reichen beim Einsatz des technologiepolitischen Instrumentariums vielfach über eEurope hinaus. Informations- und Kommunikationstechnologien (IKT) haben in der Technologiepolitik dieser Länder einen besonderen Stellenwert und die eingesetzten Instrumente zur Stimulierung von Innovation und Diffusion weisen dabei ein breites Spektrum auf:

Die Förderung von Forschung und Entwicklung greift z.B. auf spezifische Forschungsprogramme (z.B. LINK) oder die Schaffung breitbandiger Kommunikationsinfrastrukturen für Forschung (z.B. GigaPort) zurück.

Eine Verstärkung der Innovationsaktivitäten soll auch durch die Schaffung innovativer Milieus auf regionaler bzw. lokaler Ebene erreicht werden; Beispiele für entsprechende Initiativen, die auch - aber nicht nur - im Zusammenhang mit IKT eine Rolle spielen, finden sich in Großbritannien (Innovative Cluster Fund) und Dänemark (Digitales Nordjütland).

Die Stimulierung von Innovationsaktivitäten bei IKT beschränkt sich nicht notwendigerweise auf bestehende Firmen, sondern schließt eine Anhebung der Anzahl von "innovativen“ Firmengründungen ein; Ansätze, die über Bereitstellung von Venture Capital hinausreichen, finden sich beispielsweise in den Niederlanden (Twinning) und Dänemark (Innovationsmiljøer).

Zur Erhöhung der Verbreitung von IKT-Anwendungen (z.B. Internet, e-Commerce) sind vielfach Informationsdefizite bei Nicht-Anwendern zu beheben. Kampagnen, (kostenlose) Erstberatungen, Schulungen, Auszeichnungen gelten in den untersuchten Ländern als probates Mittel. Ähnlich wie Großbritannien hat Deutschland durch den Aufbau regionaler Kompetenzzentren Akzente gesetzt.

Verwaltung und Behörden können zur Verbreitung neuer Kommunikationsdienste und Anwendungen einen wesentlichen Beitrag leisten. In allen vier untersuchten Ländern werden beispielsweise Anstrengungen zur elektronischen Durchführung des Beschaffungswesens verstärkt.

Rechtliche Rahmenbedingungen für den Einsatz von IKT beziehen sich derzeit weniger auf die Regulierung des Telekommunikationssektors als auf den Aufbau einer Sicherheitsinfrastruktur im elektronischen Raum. Durch Pilotprojekte zur Verwendung digitaler Signatur im Vorfeld der Gesetzgebung hat die dänische Regierung bereits 1998 eine technologiepolitisch bedeutsame Initiative gesetzt.

\footnotetext{
${ }^{35}$ Siehe Dachs - Knoll (2001).
} 


\section{Das österreichische Universitätssystem}

Die Universitäten sind ein zentraler Bestandteil des österreichischen Innovationssystems. Ihre Bedeutung ergibt sich zum einen aus dem großen Anteil, den die Universitäten an den öffentlichen Forschungsausgaben haben, zum anderen aus den Outputs der Universitäten. Diese bestehen zum einen in der Ausbildung von qualifiziertem wissenschaftlichem Nachwuchs und Arbeitskräften, zum anderen in ihrer Funktion als größte Einrichtungen der Grundlagenforschung und damit verbundenen wissenschaftlichen Publikationen und darüber hinaus in Inputs für die Innovationsleistungen der Unternehmen (Auftragsforschung, Kooperationen etc).

Im folgenden wird ein Überblick über zentrale Bestandsdaten des österreichischen Universitätssystems (Finanzierung, Personal) gegeben sowie Indikatoren zum wissenschaftlichen und innovationsrelevanten Output (Absolventen, Publikationen, Kooperationen) präsentiert.

Die Auswertungen wurden, so weit möglich, auf der Ebene der Fakultäten durchgeführt. Die folgende Tabelle 8-1 zeigt die Zuordnung der einzelnen Fakultäten auf die Universitäten.

Tabelle 8-1: Fakultätszuordnung

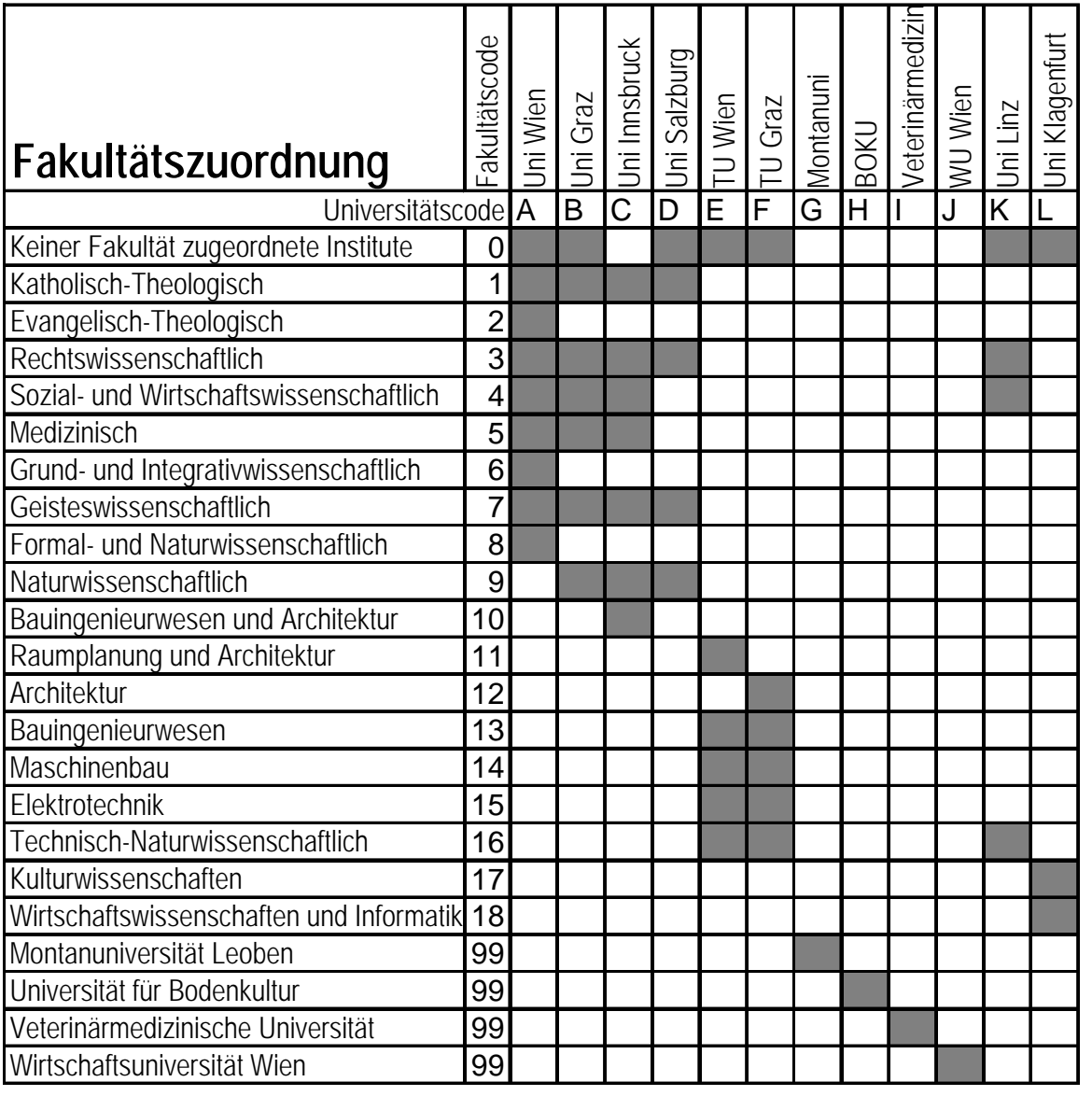

Quelle: BMBWK. Die Fakultätsbezeichnungen entsprechen dem Berichtszeitraum und wurden zwischenzeitig teilweise geändert. 


\subsection{Budget}

Abbildung 8-1: Budget und Absolventen nach Fakultäten/Universitäten,

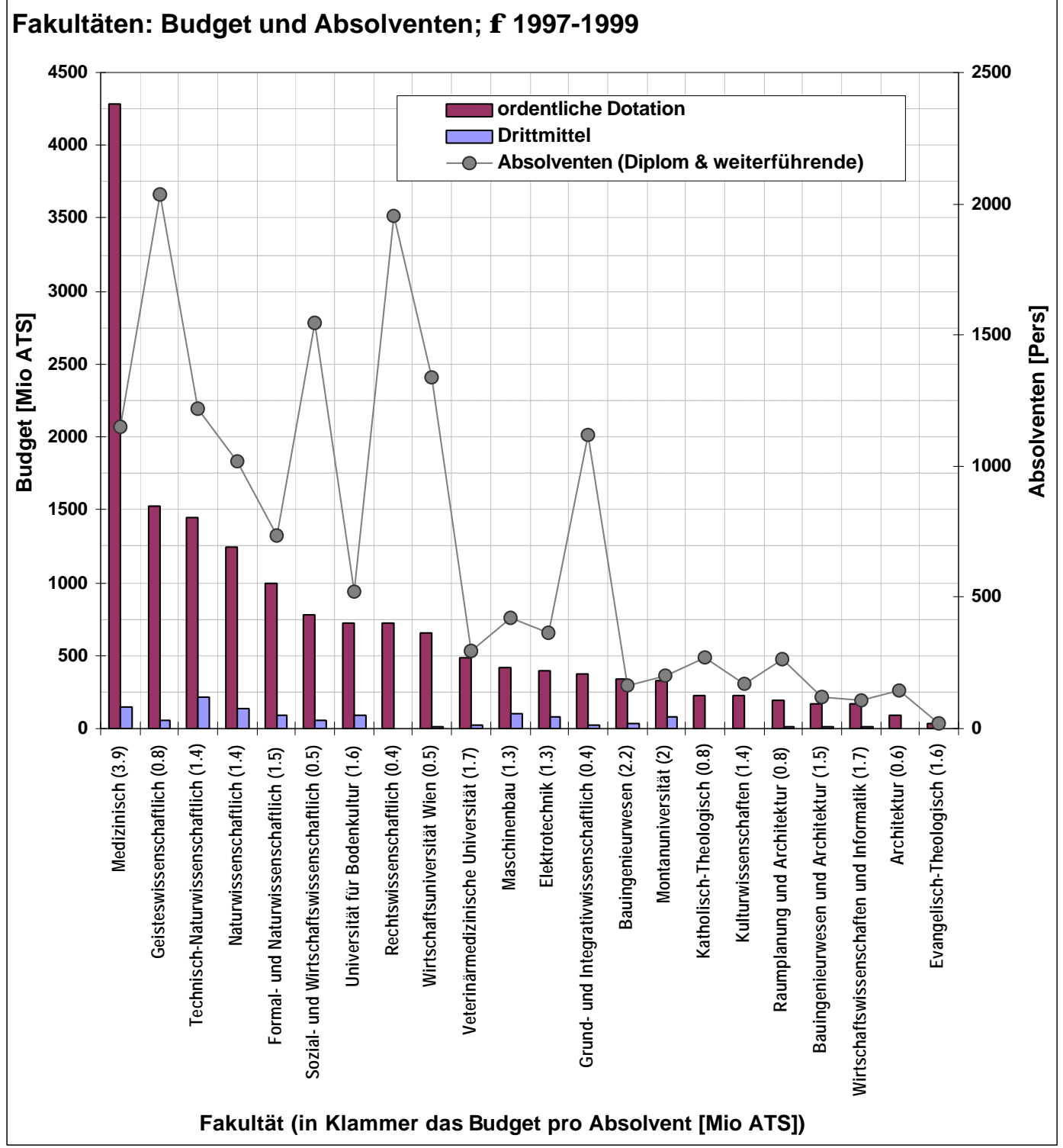

Quelle: BMBWK, Berechnungen von tip

Die höchste Budgetausstattung ${ }^{36}$ weist die Medizinische Fakultät (hier sind allerdings die Universitätskliniken mit berücksichtigt), gefolgt von den Geisteswissenschaften, auf. Interessanter als die absoluten Zahlen sind allerdings die auf die Absolventen gezogenen Werte: das bei weitem höchste Budget dabei hat die Medizin (allerdings wieder inklusive der Universitätskliniken, die ja auch in hohem Maße Dienstleistungen erbringen), gefolgt von den Bauingenieuren, der Montanuniversität Leoben und der Veterinärmedizin (2,2; 2,2 bzw. 1,6 Mio. ATS pro Absolvent). Die geringsten Budgets pro Absolvent weisen die Grund- und Integrativwissenschaften, die Rechtswissenschaften und die Wirtschaftsuniversität Wien auf ( 0,4 bis 0,5 Mio. ATS pro Absolvent). Die unterschiedlichen errechneten Ausgaben pro Absolventen sind das Resultat divergierender Kapitalintensitäten und lassen sich nicht als „Effizienzmaß" interpretieren.

\footnotetext{
${ }^{36}$ Fakultäts-Mittel sind je nach Universität verschieden und beinhalten nicht aufteilbare Kosten NICHT!
} 
Abbildung 8-2: Budgetstruktur nach Fakultäten/Universitäten, $\varnothing$ 1997-99

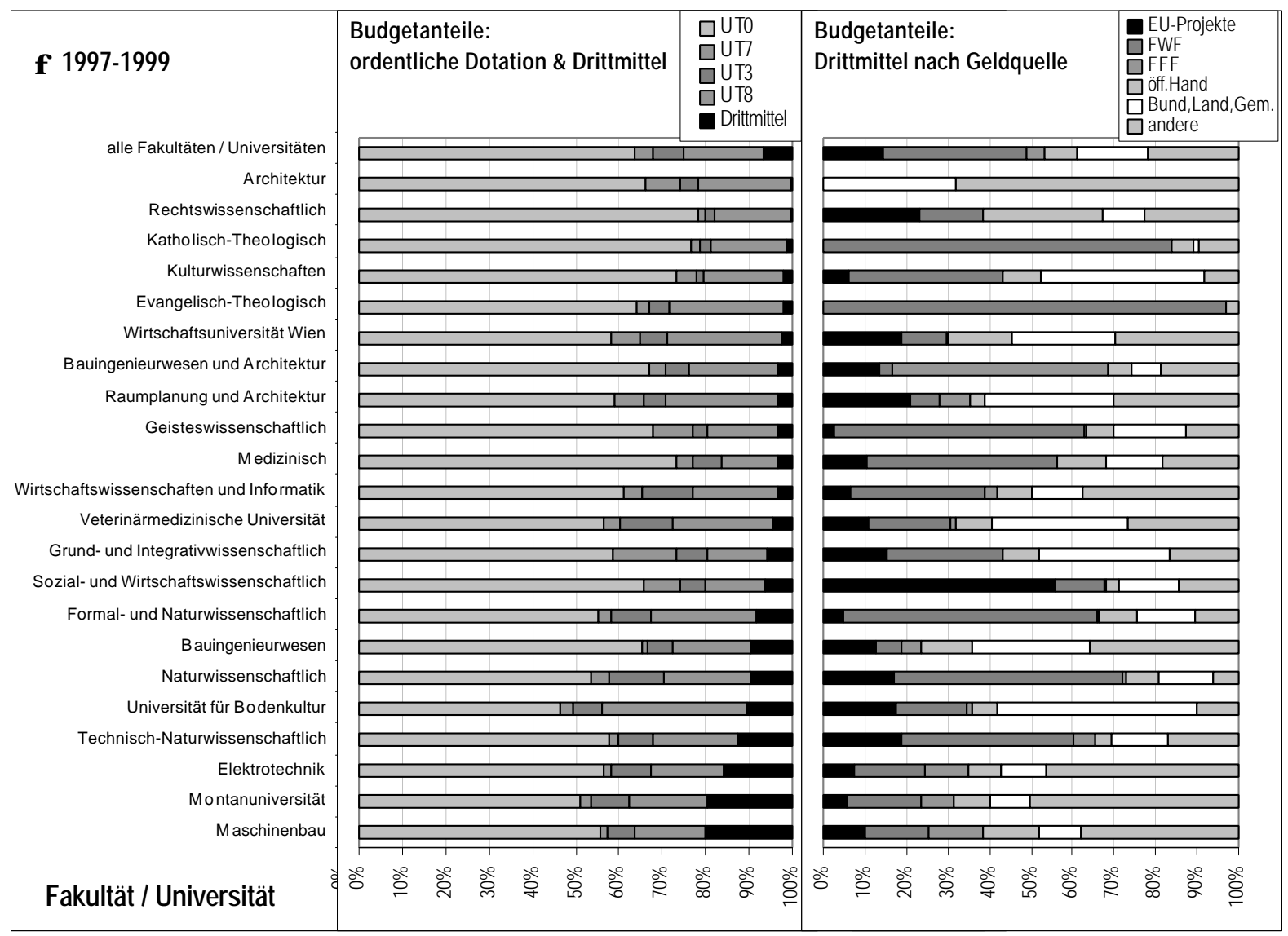

Quelle: BMBWK, "Berechnungen von tip" ${ }^{37}$

Bei der Struktur des Gesamtbudgets zeigt sich ein recht klarer Zerfall in "harte" und "weiche" Wissenschaften: die erste Gruppe weist Drittmittelanteile von 10-20\% auf (Spitzenreiter sind der Maschinenbau und die Montanuniversität Leoben); die Schlusslichter Architektur (TU Graz) und Rechtswissenschaften weisen Drittmittelanteile von unter $2 \%$ auf.

EU-Projekte spielen in der Drittmittelstruktur die größte Rolle in den Sozial- und Wirtschaftswissenschaften (55\%). Überhaupt keine Rolle spielen - nicht überraschend - diese in den theologischen Fakultäten und in der Architektur an der TU Graz. Letztere weist hingegen den größten Anteil an "anderen" (nicht-öffentlichen) Finanzierungsquellen auf. Darunter ist hauptsächlich die Finanzierung durch den Unternehmenssektor zu verstehen, der Forschungsaufträge an universitäre Institute vergibt.

Private Finanzmittel spielen bei Fakultäten mit hohem Drittmittelanteil ebenfalls eine wichtige Rolle. Bei den technischen Wissenschaften wie Maschinenbau, Elektrotechnik oder der Montanuniversität Leoben macht der Unternehmenssektor nahezu die Hälfte der Drittmittel aus. Es lässt sich aber zusammenfassend sagen, dass der Anteil der Drittmittel am Budget der meisten Fakultäten bzw. Universitäten eine geringe Rolle spielt und maximal $10 \%$ des Budgets ausmacht. Über alle Fakultäten/Universitäten gerechnet stammen ca. $7 \%$ des Budgets vom privaten Sektor.

\footnotetext{
${ }^{37}$ UT: nicht zuteilbare Gemeinkosten sind in der Abbildung nicht enthalten (UT0=Personal; UT7= Aufwendungen und gesetzli-
} che Verpflichtungen; UT3=Anlagen; UT8=Aufwendungen) 


\subsection{Personalausstattung}

Abbildung 8-3: Ausstattung mit Lehrpersonal und Personalstruktur nach Fakultäten/Universitäten

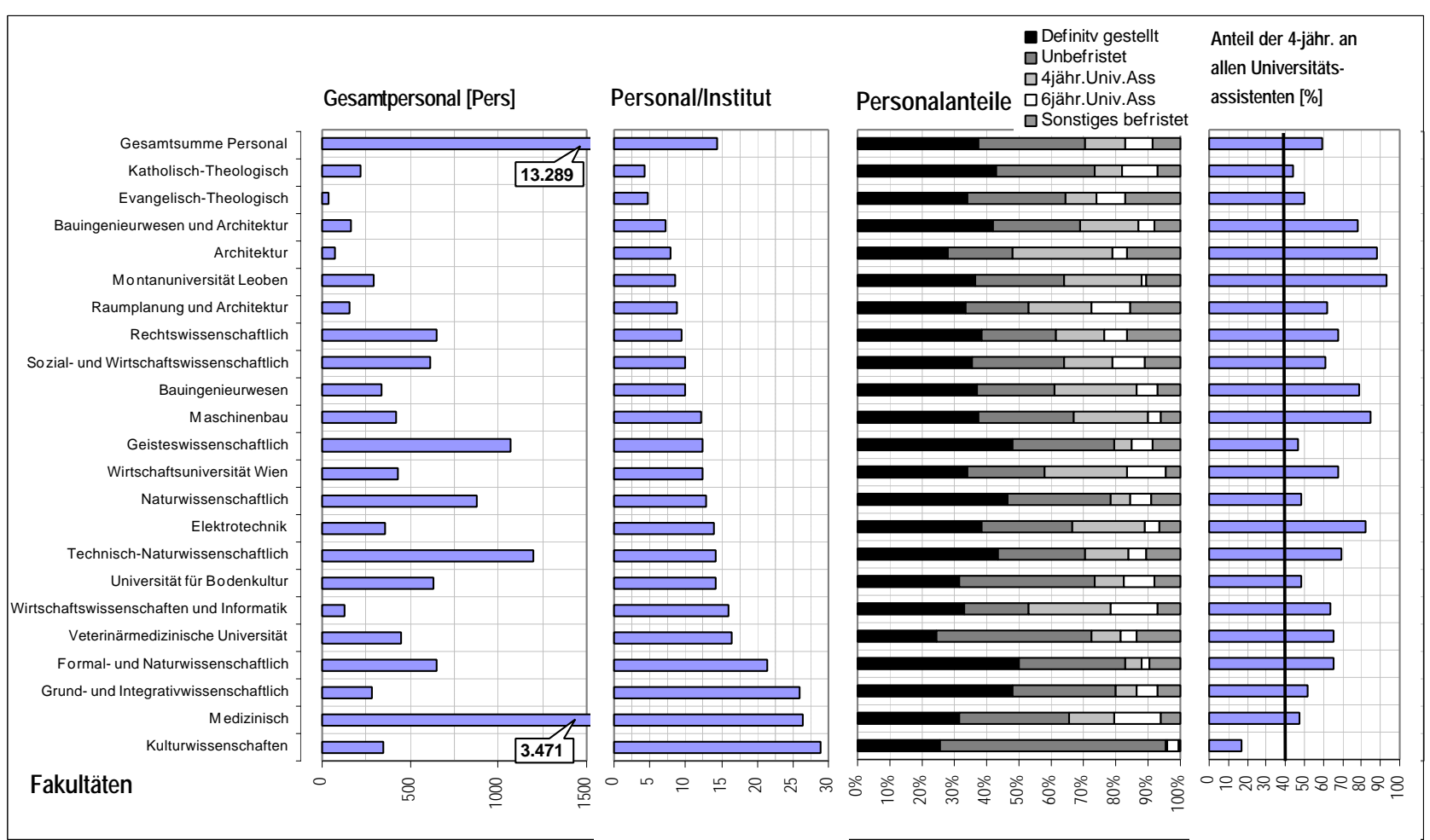

Quelle: Arbeitsberichte der Institutsvorstände, Berechnungen von tip

1999 waren 13.289 bundesbedienstete Lehrpersonen an den wissenschaftlichen Universitäten beschäftigt. Die größten Fakultäten gemessen am Personal sind in absoluten Zahlen die medizinischen, technisch-naturwissenschaftlichen und geisteswissenschaftlichen. Die personalstärksten Institute stellen die Kulturwissenschaften, Medizin und die Grund- und Integrativwissenschaftliche Fakultät; die geringste Personalausstattung weisen Bauingenieurwesen, Architektur und die theologischen Fakultäten auf.

Bei der Struktur der Personalausstattung ist zu beobachten, dass der höchste Anteil an unbefristeten und definitiv gestellten Bediensteten an den Instituten der kulturwissenschaftlichen Fakultät vorzufinden ist (Anteil $95 \%$ ), gefolgt von der formal-naturwissenschaftlichen, der grund- und integrativwissenschaftlichen, und den geistes- und naturwissenschaftlichen Fakultäten (Anteile zwischen 75 und 83 $\%)$. Die geringsten Anteile weisen mit um die $50 \%$ Architektur und Wirtschaftswissenschaften und Informatik auf.

Die rechte Grafik zeigt den Anteil der 4-jährig befristeten an der Gesamtzahl der Universitätsassistenten (4- und 6-jährige) auf: bei einer vollständigen Übernahme aller 4-jährigen in eine nachfolgende 6jährige Befristung sollte im Gleichgewichtszustand dieser Anteil bei $40 \%$ liegen; je höher daher der Anteil der 4-jährig befristeten, umso geringer die Wahrscheinlichkeit, dass diese Übernahme erfolgt. Es zeigt sich, dass tendenziell die technischen Fakultäten die geringsten Übernahmewahrscheinlichkeiten aufweisen (Spitzenreiter ist die Montanuniversität Leoben: nur $6 \%$ aller Universitätsassistenten sind im 6-jährigen Beschäftigungsverhältnis). Da die Gesamtzahl der Assistentenstellen beschränkt ist, ist davon auszugehen, dass vor allem von solchen Instituten mit größerem Anteil an 4-jährigen Assistenten auch eine größere Anzahl an wissenschaftlich ausgebildeten Personen in den nichtakademischen Sektor wechseln werden. 


\subsubsection{Universitätsjahresrechnung 2001}

\section{Bundespersonal an Instituten und Kliniken nach Wissenschaftszweigen und Beschäftigten-}

kategorien (Tabelle 15 im statistischen Anhang)

Von Statistik Austria (Bundesanstalt Statistik Österreich) wurden für den universitären Bereich auf der Grundlage des nach Instituten (bzw. Kliniken) untergliederten Stellenplans für das wissenschaftliche und nichtwissenschaftliche Personal zum Stichtag 1. 1. 2001 unter Heranziehung der aus der F\&EErhebung 1998 stammenden Koeffizienten (Arbeitszeitverteilung) Vollzeitäquivalentwerte für F\&E, für Lehre und Ausbildung sowie für sonstige Tätigkeiten nach Wissenschaftszweigen (für das Bundespersonal) ermittelt. Planstellen an den Universitäten der Künste sind in dieser Auswertung nicht berücksichtigt.

Demnach waren zum Stichtag 1. 1. 2001 an Österreichs Universitäten insgesamt 6820,2 Vollzeitäquivalente für F\&E eingesetzt, welche sich wie folgt auf die Wissenschaftszweige verteilten:

\begin{tabular}{|l|c|c|}
\hline Wissenschaftszweige & VZÄ für F\&E & in Prozent \\
\hline Naturwissenschaften & 1816,9 & 26,6 \\
\hline Technische Wissenschaften & 914,9 & 13,4 \\
\hline Humanmedizin (einschl. Kliniken) & 1886,2 & 27,7 \\
\hline Land- und Forstwirtschaft, Veterinärmedizin & 340,1 & 5,0 \\
\hline Sozialwissenschaften & 1132,8 & 16,6 \\
\hline Geisteswissenschaften & 729,3 & 10,7 \\
\hline INSGESAMT & 6820,2 & 100,0 \\
\hline
\end{tabular}

Es sei darauf hingewiesen, dass die zugrundeliegenden Planstellenwerte, die natürlich auch unbesetzte Planstellen einschließen, das Instituts- bzw. Klinikpersonal umfassen, während das Personal der zentralen Universitätsverwaltungen ausgeschlossen ist und auch refundiertes Personal bzw. an den Instituten (Kliniken) tätiges Nicht-Bundespersonal gleichfalls nicht einbezogen ist. 


\subsubsection{Lehrpersonal und Frauenanteil}

Abbildung 8-4: Frauenanteil nach Lehrpersonalkategorien

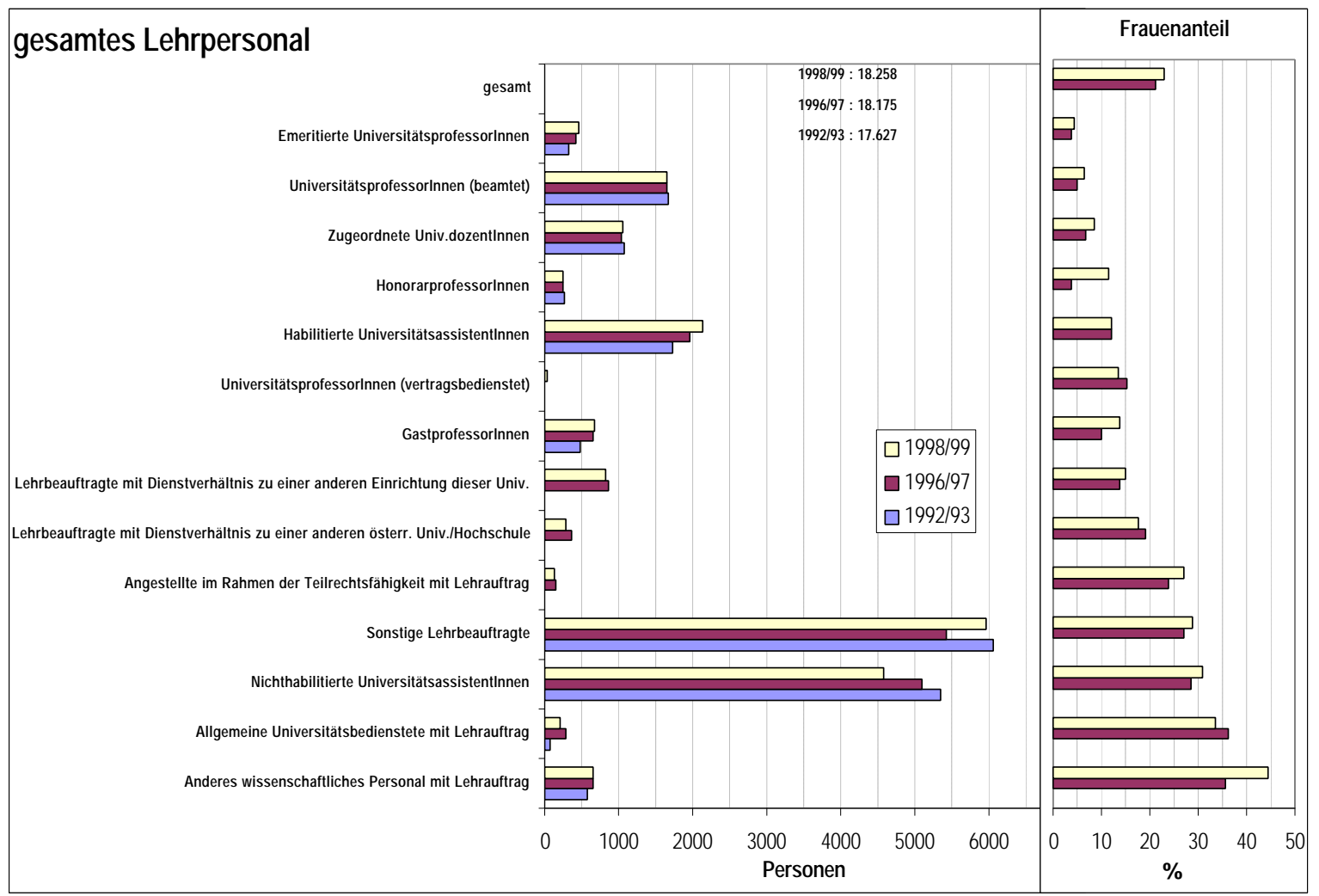

Quelle: BMBWK, Berechnungen von tip

Die Personalkategorien sind nach ihrem Frauenanteil im Jahr 1999 gereiht dargestellt. Diese Reihung spiegelt recht gut die Hierarchie des Lehrpersonals bzw. die historische Entwicklung wider: der geringste Frauenanteil ist mit unter $5 \%$ bei den Emeritierten festzustellen; die höchsten Frauenanteile bestehen bei nichthabilitierten Lehrenden. Für praktisch alle Kategorien zeigt sich im Vergleich 1996/97 mit 1998/99 ein wachsender Frauenanteil. ${ }^{38}$

Zusammenfassend kann angemerkt werden, dass der Frauenanteil mit zunehmender Stufe der akademischen Hierarchie sinkt: begonnene Erststudien - $55 \%$, abgeschlossene Erststudien - $50 \%$, begonnene weiterführende Studien - $45 \%$, abgeschlossene weiterführende Studien - $35 \%$, nichthabilitierte Universitätsassistentenschaft - $32 \%$, habilitierte Assistentenschaft - $15 \%$, Professorenstand $-7 \%$, Emeritierte - $4 \%$.

\footnotetext{
${ }^{38}$ Für die Jahre davor sind die Daten nicht nach Geschlechtern differenziert. Quelle: Arbeitsberichte der Institutsvorstände
} 


\subsection{Studierende und Studienabschlüsse: Basis für den Forschungsnachwuchs}

\subsubsection{Gesamtübersicht}

Abbildung 8-5: Entwicklung der begonnenen/belegten/abgeschlossenen Studien

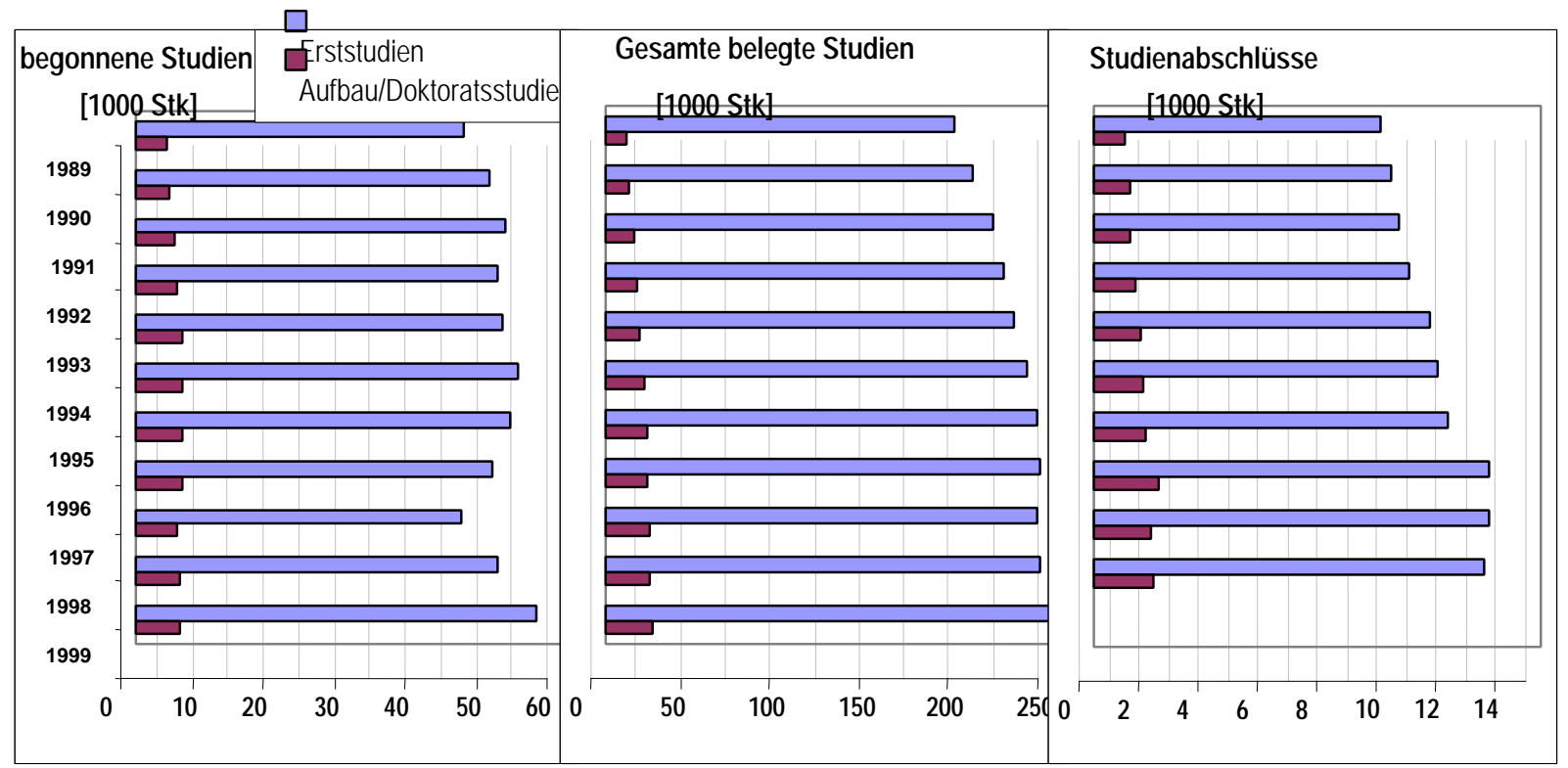

Quelle: BMBWK, Berechnungen von tip

Die Abbildung 8-5 zeigt die Entwicklung der begonnenen und gesamten belegten Studien sowie der Studienabschlüsse im Zeitraum 1989 - 1999, jeweils getrennt nach Erststudien (Diplom- und Kurzstudien) und weiterführenden Studien (Aufbau- und Doktoratsstudien). ${ }^{39}$ Die Zahlen stellen von In- und Ausländerlnnen begonnene, belegte bzw. abgeschlossene Studien dar und nicht die Anzahl der Studierenden, die ein Studium beginnen, belegen oder anschließen; Mehrfachstudien werden entsprechend mehrfach gezählt. Die Jahreszahlen bezeichnen den Beginn des jeweiligen Studienjahres (Jahr $1995=$ WS 1995/96 + SS 1996).

Die begonnenen Studien, sowohl Erststudien wie auch weiterführende Studien, weisen nach einem Rückgang in den Jahren 1995-1997 wieder steigende Tendenz auf. Diese Tendenz kann in sehr abgeschwächter Form auch bei den belegten Studien und (mit einem Jahr Verzögerung) den Studienabschlüssen festgestellt werden. Ein Teil dieses Rückgangs bei den begonnenen Studien kann mit der Einführung der Fachhochschulen 1994 erklärt werden. Diese wiesen 1998 etwa 3.000 begonnene Studien auf und zeigen eine stark wachsende Tendenz. Allerdings zeigt die wieder steigende Zahl von begonnenen Studien auch, dass die zunehmende Attraktivität von Fachhochschulen nicht (nur) auf Kosten der Universitäten geht, sondern auch eine Erweiterung der Nachfragebasis nach tertiärer Bildung induziert.

Stärker gewachsen sind die weiterführenden Studien: im Berichtszeitraum 1989 - 1999 erhöhte sich die Zahl der begonnenen weiterführenden Studien um 34 \% (zum Vergleich: Erststudien +22 \%), die Abschlüsse weisen mit $+91 \%$ fast eine Verdopplung auf (zum Vergleich: Erststudienabschlüsse $+37 \%$ ).

\footnotetext{
${ }^{39}$ Für das Jahr 1999 standen für die Studienabschlüsse noch keine endgültigen Zahlen zur Verfügung.
} 


\subsubsection{Studienabschlüsse nach Fakultäten}

Abbildung 8-6: Veränderung der Studienabschlüsse 1989-92 / 1996-98

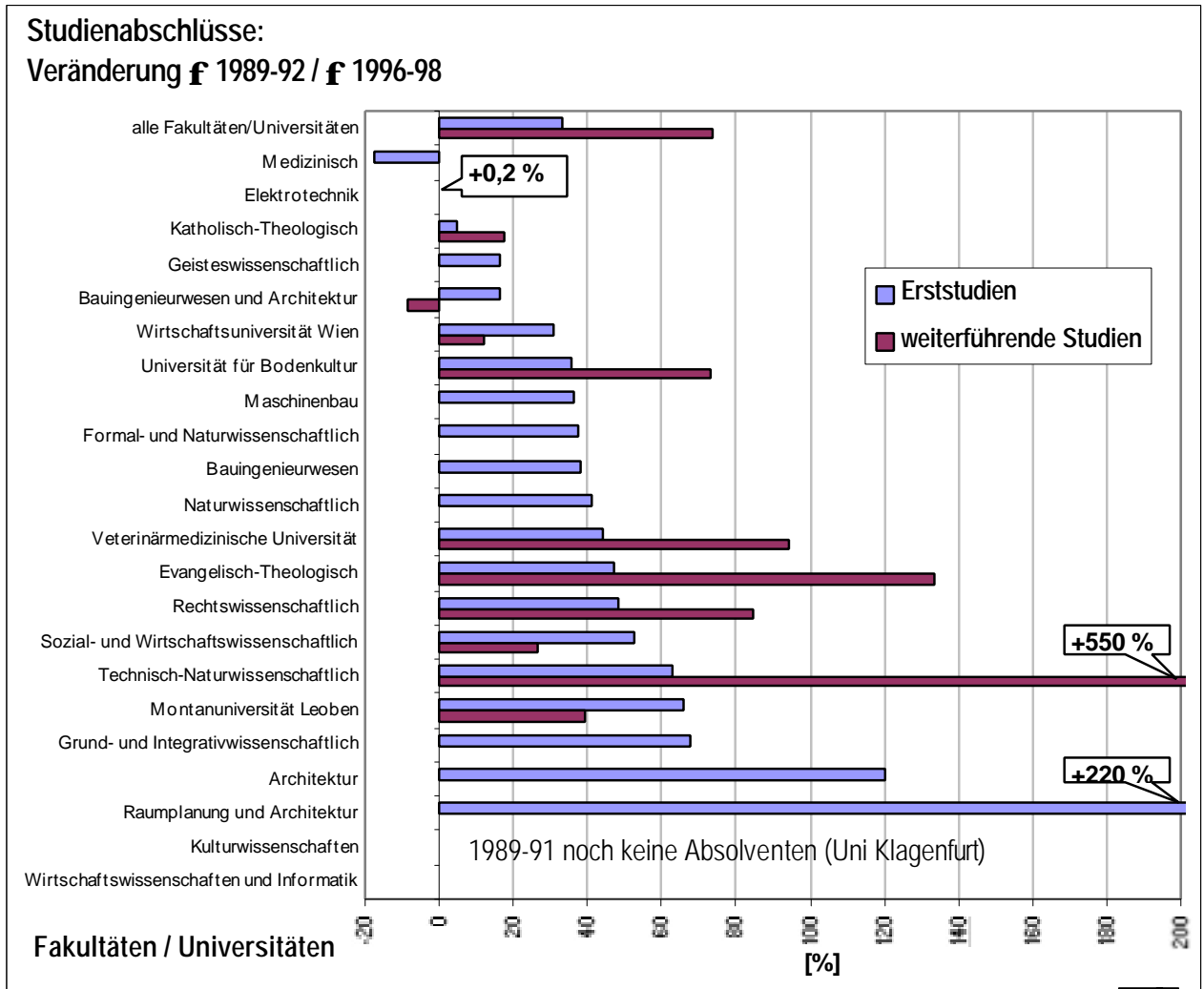

Quelle: BMBWK, Berechnungen von tip

Bezüglich der Studienabschlüsse lässt sich festhalten, dass mit Ausnahme der Medizin sämtliche Fakultäten steigende Studienabschlüsse verzeichnen. An der Spitze bei Erststudienabschlüssen liegt dabei die Fakultät "Raumplanung und Architektur" an der TU Wien mit einer Steigerung von $220 \%$ zwischen den beiden Zeitperioden. Bei weiterführenden Studien liegen die technischnaturwissenschaftlichen Fakultäten an der Spitze, gefolgt von den evangelisch-theologischen Fakultäten und der Veterinärmedizinischen Universität. Die Ingenieurwissenschaften verzeichnen, mit Ausnahme der Montanuniversität Leoben, ein unterdurchschnittliches Wachstum in den Studienabschlüssen. 


\subsection{Internationale Mobilität der österreichischen Studierenden}

\section{Abbildung 8-7: Auslandsaufenthalte von ErststudienabsolventInnen}

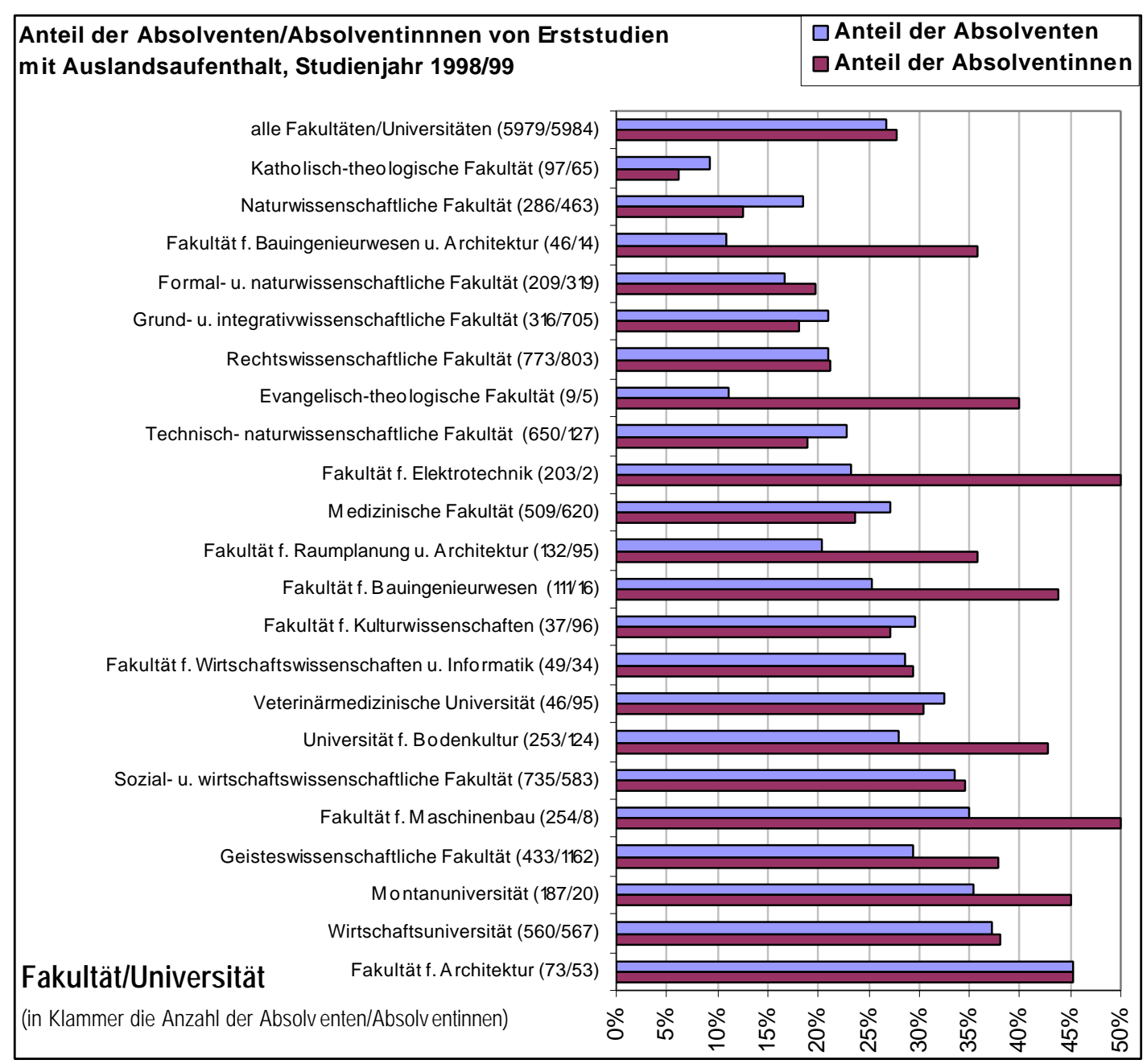

Quelle: STATISTIK AUSTRIA, Berechnungen von tip ${ }^{40}$

Die Abbildung zeigt den Anteil von Absolventen und Absolventinnen von Erststudien, die während ihres Studiums mindestens einen Auslandsaufenthalt absolviert haben (die Fakultäten sind nach dem Gesamtanteil, d.h. Absolventen und Absolventinnen, gereiht).

Den höchsten Anteil an Auslandsaufenthalten weisen demnach die an der TU Graz eingerichtete Fakultät für Architektur (45\%) und die Wirtschaftsuniversität Wien (37\%) auf; die geringsten Anteile finden sich bei der katholisch-theologischen Fakultät $(8 \%)$ und der naturwissenschaftlichen Fakultät (15\%).

Die Unterschiede zwischen Absolventinnen und Absolventen sind in den meisten Fällen gering; auffallend ist, dass bei größeren geschlechtsspezifischen Unterschieden meist die Absolventinnen einen höheren Anteil an Auslandsaufenthalten aufweisen (Extreme: Bauingenieurwesen und Architektur: 36 vs. $11 \%$, Elektrotechnik: 50 vs. $23 \%$ ).

\footnotetext{
${ }^{40}$ Die Zahlen beruhen auf einer Auswertung des Fragebogens, der von der Statistik Austria an alle Absolventen österreichischer Universitäten verteilt wird. Ein Vergleich mit den Absolventenzahlen ergibt, dass die Rücklaufquote bei etwa $90 \%$ im Falle der Erststudienabschlüsse und etwa $80 \%$ bei den weiterführenden Studien liegt.
} 


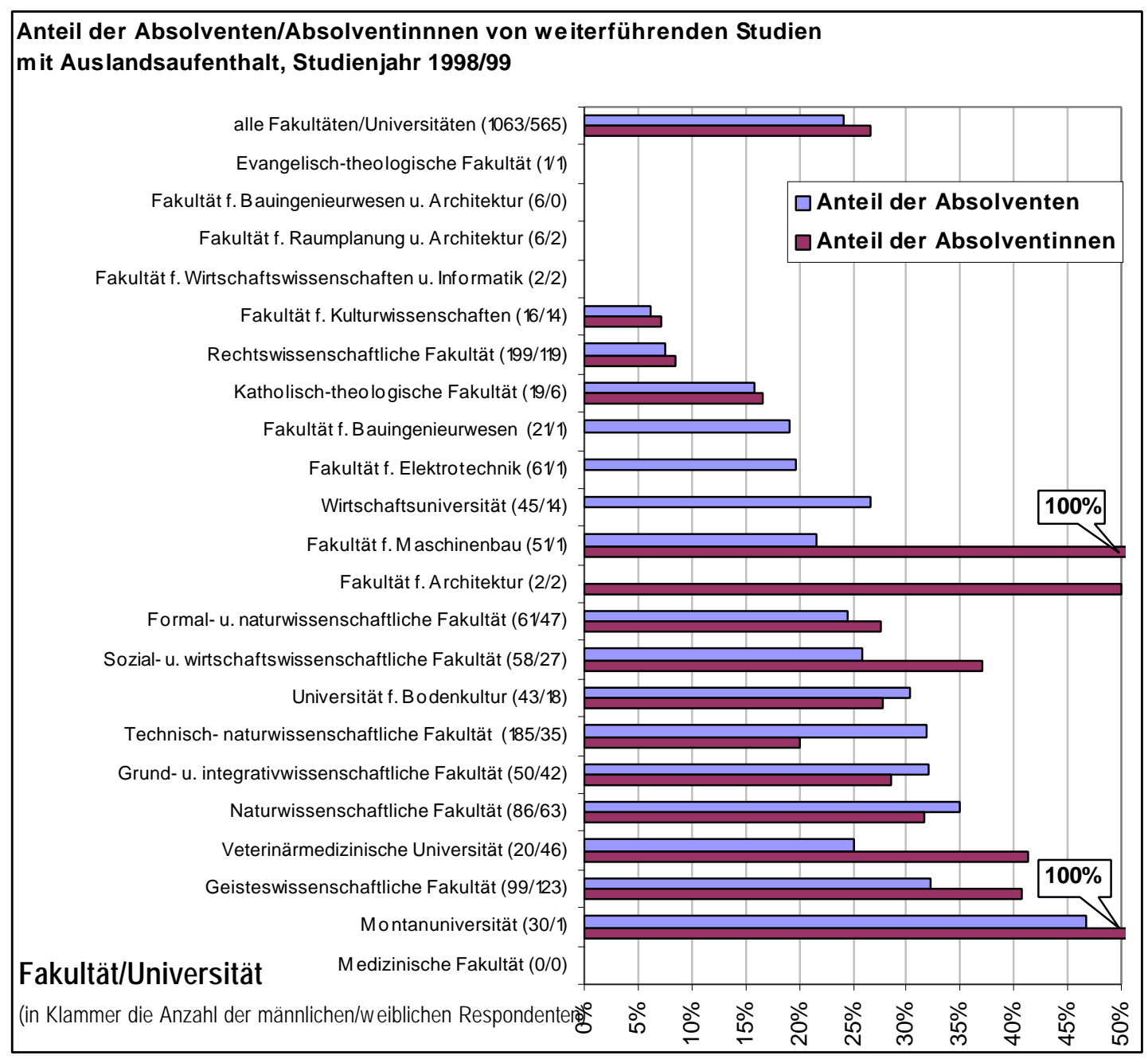

Quelle: STATISTIK AUSTRIA, Berechnungen von tip

Die Abbildung 8-8 zeigt den Anteil von Absolventen und Absolventinnen von weiterführenden Studien, die während ihres Studiums mindestens einen Auslandsaufenthalt absolviert haben (die Fakultäten sind nach dem Gesamtanteil, d.h. Absolventen und Absolventinnen, gereiht).

Im Vergleich mit den Erststudienabschlüssen ist die Reihenfolge der Fakultäten hier doch recht unterschiedlich: Wirtschaftsuniversität Wien und Architektur (TU Graz) nehmen keine Spitzenplätze mehr ein (nur $27 \%$ der Absolventen und keine einzige Absolventin der WU Wien weisen einen Auslandsaufenthalt auf; auf der Fakultät für Architektur (TU Graz) hingegen wies keiner ihrer 2 Absolventen, aber beide Absolventinnen einen Auslandsaufenthalt auf). Die AbsolventInnen von 4 Fakultäten können auf überhaupt keinen Auslandsaufenthalt verweisen (diese 4 weisen allerdings nur sehr kleine Absolventenzahlen auf). Für die Medizin schließlich liegen keine Ergebnisse vor (die Medizin kennt kein gesondertes Doktoratsstudium).

Spitzenreiter ist bei den weiterführenden Studien die Montanuniversität Leoben (fast $50 \%$ der AbsolventInnen), gefolgt von den Geisteswissenschaften (32\% der AbsolventInnen). 


\subsubsection{Förderungsprogramme für den wissenschaftlichen Nachwuchs}

Tabelle 8-2: Beteiligung an Förderprogrammen für den wissenschaftlichen Nachwuchs

\begin{tabular}{|c|c|c|c|c|}
\hline Stipendium/Preis & Geber & \begin{tabular}{|r|} 
Teilnehmer \\
$\Sigma 1998-2000$ \\
$* \sum 1999-2000$ \\
\end{tabular} & $\begin{array}{r}\phi \text { Budget/Stip } \\
\text { [Tsd ATS] }\end{array}$ & Kriterien \\
\hline Erwin Schrödinger-Auslandsstipendium & FWF & 297 & 417 & für Graduierte/Wissenschafter, 10-24 Monate, ATS 290-500 t/Jahr \\
\hline Erwin Schrödinger-Rückkehrprogramm & FWF & 1 & 1,700 & für Graduierte/Wissenschafter, max.ATS 4 Mio \\
\hline Lise Meitner-Programm für Ausländerlnnen & FWF & 50 & 408 & für ausländische Postdocs unter 35,12 Monate, bis ATS $210 \mathrm{t}$ \\
\hline Charlotte Bühler-Programm & FWF & 31 & 768 & weibl.Hochschullehrernachwuchs unter 41, 12-24 Monate, ATS 530/Jahr \\
\hline Herta Firnberg-Stellen & FWF & 35 & 1,891 & weibl.wiss.Nachwuchs unter 40 (Postdoc), in Ö lebend, 3 Jahre, ATS 662t/Jahr \\
\hline START-Programm & FWF & 15 & & ForscherInnen unter 35, max 6.Jahre, ATS 2-2,5 Mio/Jahr \\
\hline Wittgenstein-Preis & FWF & 6 & 5,920 & ForscherInnen, ATS 20 Mio \\
\hline APART (Aus.Progr.f.Advanced Res\&Techn.) & ÖAW & 55 & & für Habilitation, 3 Jahre, ATS 620 t/Jahr \\
\hline DOC (Doktorandenprogramm) & ÖAW & 98 & 885 & Doktoranden, max.2 Jahre, ATS 200 t/Jahr \\
\hline Öst.Progr.f.Techn.Doktoranden bei CERN & BMBWK & $16^{*}$ & & max.24 Monate bei CERN, durchschn. ATS 36t/Monat \\
\hline Beihilfe für Zwecke der Wissenschaft & BMBWK & k.A. & & Vergabe im Unibereich, Summe 1999/2000: ATS 17 Mio \\
\hline Praktika bei internat/supranat.Organisationen & BMBWK & 101 & 420 & \\
\hline Postgrad.Stipendien für das fremdspr.Ausl. & BMBWK & $270^{\star}$ & 182 & \\
\hline Kurt Gödel-Stipendien für d.Int.Space Univ. & BMBWK & $19^{*}$ & 75 & Postdocs/Wissenschafter, max.12 Monate, max ATS 12.500/Monat \\
\hline Forschungsstip.am Hist.Inst. beim ÖKI Rom & BMBWK & $22^{\star}$ & & ATS 12.000/Monat \\
\hline
\end{tabular}

Quelle: BMBWK, Berechnungen von tip

Tabelle 8-2 gibt einen Überblick über die wichtigsten Stipendienprogramme für junge Forscher und Forscherinnen (2 davon, das Charlotte Bühler-Programm und die Herta Firnberg-Stellen, richten sich explizit an Forscherinnen). Im Jahr 2000 wurden insgesamt 264 Stipendien bzw. Preise vergeben, der Gesamtfinanzierungsbetrag betrug in diesem Jahr etwa ATS 230 Mio.

Auf europäischer Ebene ist im 4. Rahmenprogramm der EU besonders das Programm „Förderung und Mobilität von Wissenschaftern" (TMR) zu erwähnen, da es sich explizit an Hochschulen richtete und die grenzüberschreitende Mobilität des wissenschaftlichen Nachwuchses förderte. Im TMR Programm konnten nach dem bottom-up Prinzip aus allen Bereichen der Natur- und Ingenieurswissenschaft sowie der Geistes-, Sozial- und Wirtschaftswissenschaften Anträge gestellt werden, sofern sie den generellen Zielsetzungen des Rahmenprogramms entsprachen. Zielgruppen waren vor allem graduierte und promovierte AkademikerInnen sowie Forschungseinrichtungen, die einen Ausbildungsplatz für qualifizierte AkademikerInnen zur Verfügung stellen. Diese Aktionslinie wurde im 4. Rahmenprogramm in vier Aktionsbereiche untergliedert und ging im 5. Rahmenprogramm in die spezifische Maßnahme „Ausbau des Potentials an Humanressourcen in der Forschung“ über.

Tabelle 8-3: Förderung der Mobilität in den EU-Rahmenprogrammen

\begin{tabular}{|l|l|l|l|}
\hline 4. Rahmenprogramm & 5. Rahmenprogramm \\
\hline & $\begin{array}{l}\text { Erfolgreiche Beteili- } \\
\text { gungen }\end{array}$ & $\begin{array}{l}\text { Erfolgreiche Beteiligun- } \\
\text { gen }\end{array}$ & 30 \\
\hline Forschungsnetzwerke (NW) & 53 & Ausbildungsnetze & 35 \\
\hline Zugang zu Großforschungsanlagen (LSF) & 1 & Stipendien & 34 \\
\hline Forschungsstipendien (RTG) & 81 & Konferenzen & \\
\hline Begleitmaßnahmen (AM) & 5 & & \\
\hline
\end{tabular}


Tabelle 8-4: Auslandsaufenthalte nach Personengruppen

\begin{tabular}{|c|c|c|c|c|c|c|c|c|c|c|c|c|}
\hline ? 1997-1999 (2000) & Studen & enzah & & & $\begin{array}{l}\text { Outgoir } \\
\text { nach P }\end{array}$ & $\begin{array}{l}\text { grsone } \\
\text { rns }\end{array}$ & ruppe & & $\begin{array}{l}\text { Incomir } \\
\text { nach P }\end{array}$ & $\begin{array}{l}\text { g } \\
\text { ersone }\end{array}$ & gruppe & \\
\hline Universität & 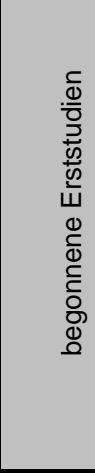 & 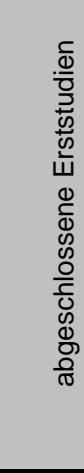 & 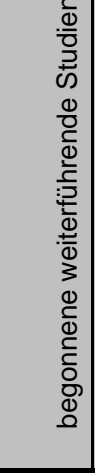 & 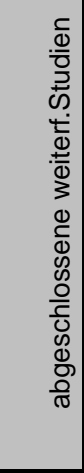 & 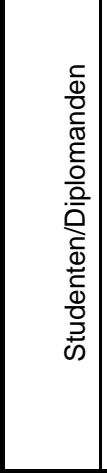 & $\begin{array}{l}0 \\
\frac{0}{0} \\
\frac{0}{0} \\
\frac{0}{0} \\
0 \\
0\end{array}$ & 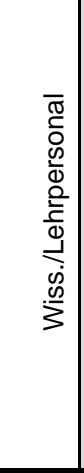 & 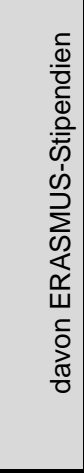 & 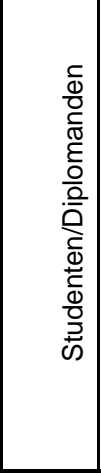 & $\begin{array}{l}\frac{0}{0} \\
\frac{0}{00} \\
\frac{0}{0} \\
\frac{0}{0} \\
0\end{array}$ & 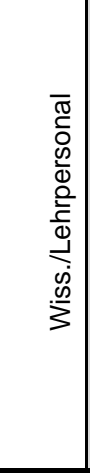 & 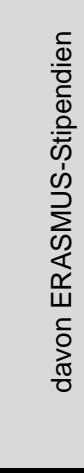 \\
\hline Universität für Bodenkultur Wien & 900 & 442 & 229 & 78 & 145 & 23 & 15 & 120 & 86 & 45 & 5 & 73 \\
\hline Universität Innsbruck & 6,447 & 1,735 & 807 & 251 & 600 & 100 & 180 & 350 & 380 & k.A. & 180 & 250 \\
\hline Universität Wien & 17,096 & 4,046 & 1,659 & 566 & 1,036 & k.A. & 200 & 680 & 584 & k.A. & 380 & 584 \\
\hline
\end{tabular}

Quelle: Auslandsabteilungen der Universitäten Innsbruck, Wien und Bodenkultur; Berechnungen von tip

Anmerkung: die anderen Universitäten wurden kontaktiert, konnten jedoch keine entsprechendes Datenmaterial liefern.

Tabelle 8-4 zeigt, dass das ERASMUS-Stipendium zu den wichtigsten Finanzierungsinstrumenten zur Förderung der Mobilität der Studierenden und Lehrenden zählt. Andere Finanzierungsquellen sind Förderungen von kurzfristigen wissenschaftlichen Arbeiten im Ausland und Joint Study-Programme (nach ERASMUS die wichtigsten Programme), CEEPUS, Partnerschaften, Kulturübereinkommen, etc. ERASMUS stellt einen Programmteil von SOKRATES dar, an dem Österreich seit 1992/93 sehr aktiv teilnimmt. Innerhalb des Studierendenaustausches wurden seit 1992 über 11.000 österreichische Studierende an die teilnehmenden Hochschuleinrichtungen entsandt.

Die Zahlen in Tabelle 8-4 stellen Mittelwerte für die Jahre 1997-1999 (bzw. 1996-1998 im Fall der Absolventenzahlen) oder jeweils aktuellste Werte dar (nicht für alle Kategorien waren Zeitreihen vorhanden). Die Mobilitätszahlen umfassen nur Bewegungen, die von den jeweiligen Auslandsstudienabteilungen erfasst wurden; insbesondere enthalten sie keine Zahlen zu privat oder z.B. über LEONARDO finanzierte Auslandsaufenthalte. Ebenso sind Auslandsaufenthalte von wissenschaftlichen Mitarbeitern, die im Rahmen von Projekten abgewickelt wurden, nicht erfasst. 


\subsection{Wissenschaftliche Produktion der Universitäten}

\subsubsection{Lehre und Betreuung}

\section{Abbildung 8-9: Lehre und Betreuung nach Fakultäten/Universitäten}

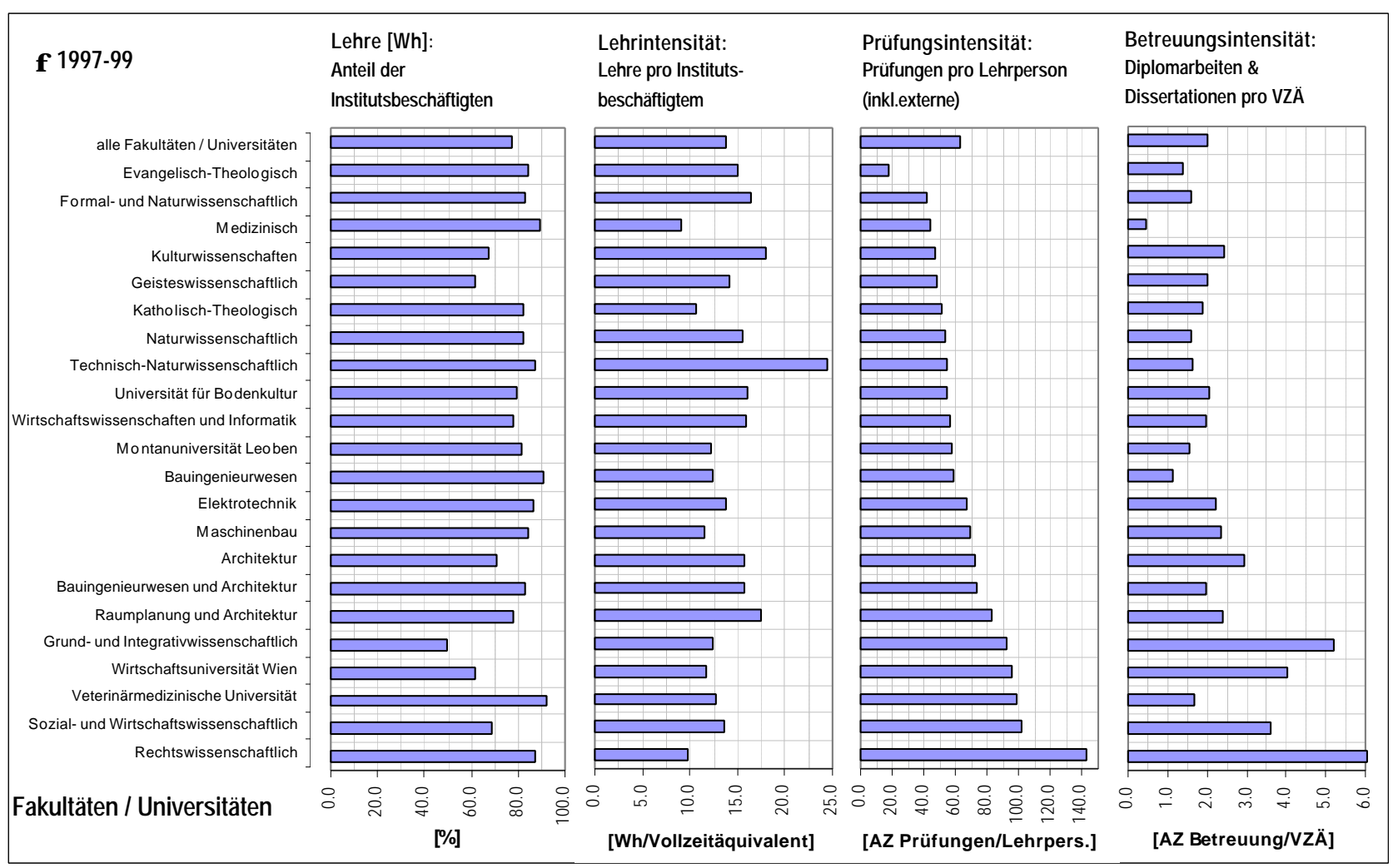

Quelle: BMBWK, Berechnungen von tip

Die erste Reihe zeigt den Anteil am gesamten Lehrangebot, das von Institutsbediensteten durchgeführt wird. Den größten Anteil an von externem Lehrpersonal abgehaltenen Stunden weist demnach die grund- und integrativwissenschaftliche Fakultät auf (mehr als $50 \%$ ), den höchsten "Eigenanteil" hat mit über $93 \%$ die Veterinärmedizin. Die übrigen Fakultäten bewegen sich zwischen 60 und rd. $90 \%$.

Die höchste Lehrintensität, d.h. die Anzahl der angebotenen Pflichtlehrveranstaltungen [Wh] pro interner Lehrperson, findet sich auf der technisch-naturwissenschaftlichen Fakultät mit fast 25 Wochenstunden pro Vollzeitäquivalent (der Schnitt über alle Fakultäten beträgt etwa $14 \mathrm{Wh}$ ); den geringsten Wert weist mit 9 Wh die Medizin auf.

Die unterschiedlichen Prüfungsmodi wurden in eine Aktivitätszahl zusammengefasst ${ }^{41}$. Die technischen Fächer finden sich in der Rangordnung der Prüfungsaktivität, bezogen auf das (interne + externe) Lehrpersonal im Mittelfeld; Spitzenreiter sind die Rechtswissenschaften, die Sozial- und Wirtschaftswissenschaften, Veterinärmedizin, Wirtschaftsuniversität, sowie Grund- und Integrativwissenschaften. Mit der Ausnahme der Veterinärmedizin stellen diese Fakultäten auch die Spitzenplätze bei der Betreuungsintensität. ${ }^{42}$

\footnotetext{
${ }^{41}$ AZ Prüfungen $=\left(\right.$ Beurteilungen $\left.{ }^{\star} 0,75\right)+($ Vorlesungsprüfungen*1 $)+\left(\right.$ Einzelprüfungen $\left.{ }^{\star} 2\right)+($ kommissionelle Prüfungen*2 $)$

${ }^{42}$ Aktivitätszahl Betreuung $=$ Diplomarbeiten*1 + Dissertationen ${ }^{*} 2$
} 


\subsubsection{Publikationen}

Abbildung 8-10: Publikationstätigkeit nach Fakultäten/Universitäten

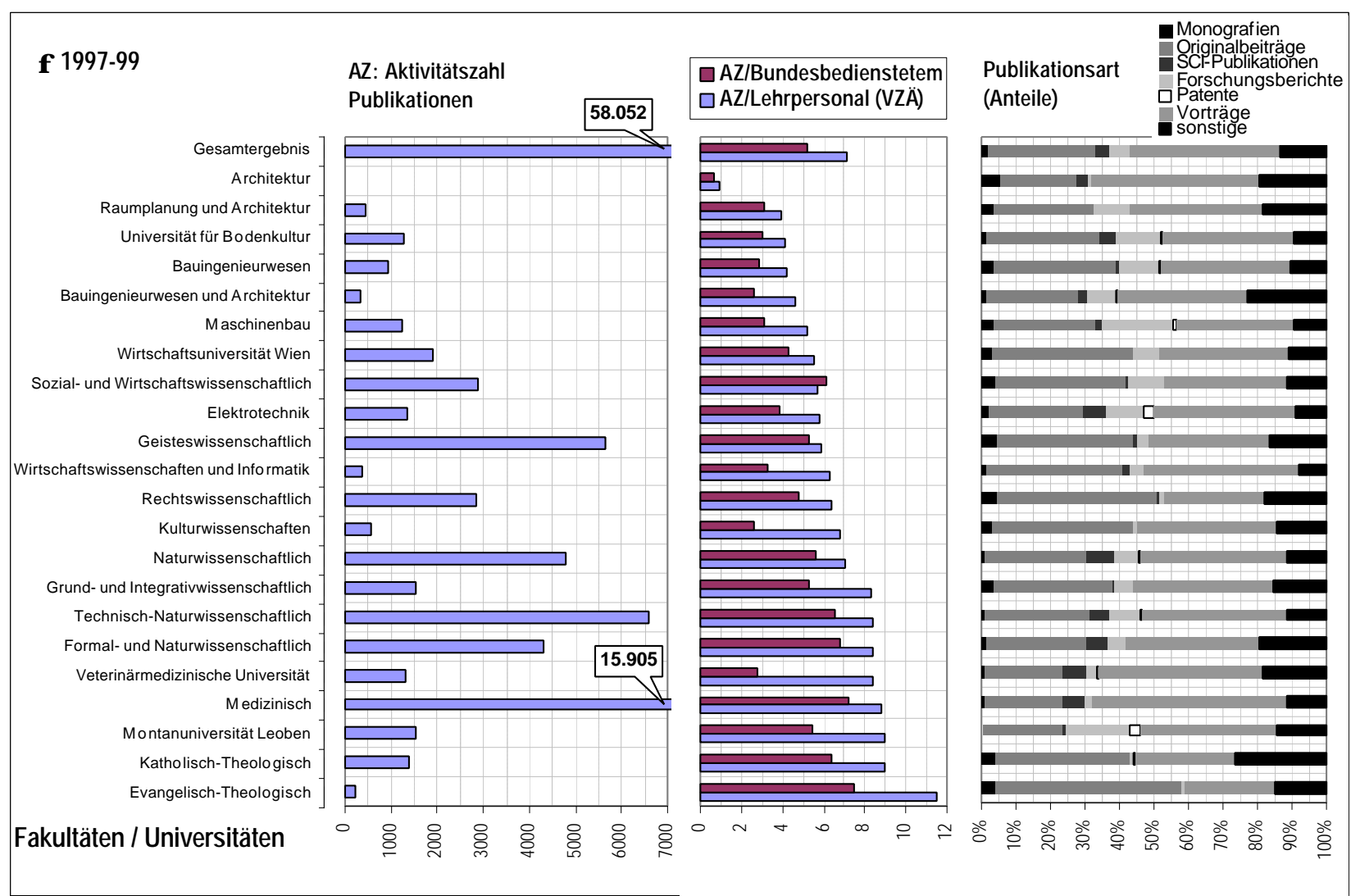

Quelle: BMBWK, Berechnungen von tip

Die Publikationen wurden in einer Aktivitätszahl zusammengefasst ${ }^{43}$. Die höchste Publikationstätigkeit, gemessen am Lehrpersonal, weisen die theologischen Fakultäten auf, gefolgt von der Montanuniversität (die damit die "produktivste" technische Fakultät/Universität darstellt) und Human- bzw. Veterinärmedizin. Schlusslichter bilden Architektur, Raumplanung und die Universität für Bodenkultur.

Zur Struktur der Veröffentlichungen kann angemerkt werden, dass Patente naturgemäß nur in den technischen Fakultäten eine Rolle spielen; nennenswerte Anteile am wissenschaftlichen Output lassen sich dabei in der Elektrotechnik und an der Montanuniversität Leoben feststellen.

Die größten Anteile an Publikationen, die im "Science Citation Index" erfasst sind, an den gesamten Originalbeiträgen stellen Human- und Veterinärmedizin, die naturwissenschaftliche Fakultät und die Elektrotechnik.

Über alle Fakultäten/Universitäten gerechnet haben Originalbeiträge in Fachzeitschriften und Sammelwerken den höchsten Anteil an der Aktivitätszahl.

\footnotetext{
${ }^{43}$ AZ Publikationen $=\left(\right.$ Erstauflagen von Monografien $\left.{ }^{*} 3\right)+\left(\right.$ Originalbeiträge in Fachzeitschriften und Sammelwerken ${ }^{*}$ (davon $\mathrm{SCI} / \mathrm{SSCl} / \mathrm{AHCl}$-Publikationen $\left.\left.{ }^{*} 3\right)\right)+\left(\right.$ Forschungsberichte $\left.{ }^{\star} 1,5\right)+\left(\right.$ Patente $\left.^{\star} 2,5\right)+($ Vorträge und Präsentationen bei wissenschaftlichen Kongressen $\left.{ }^{*} 0,5\right)+\left(\right.$ sonstige wissenschaftliche Veröffentlichungen $\left.{ }^{*} 0,5\right)$
} 


\subsubsection{Projekttätigkeit}

Abbildung 8-11: Projekttätigkeit nach Fakultäten/Universitäten

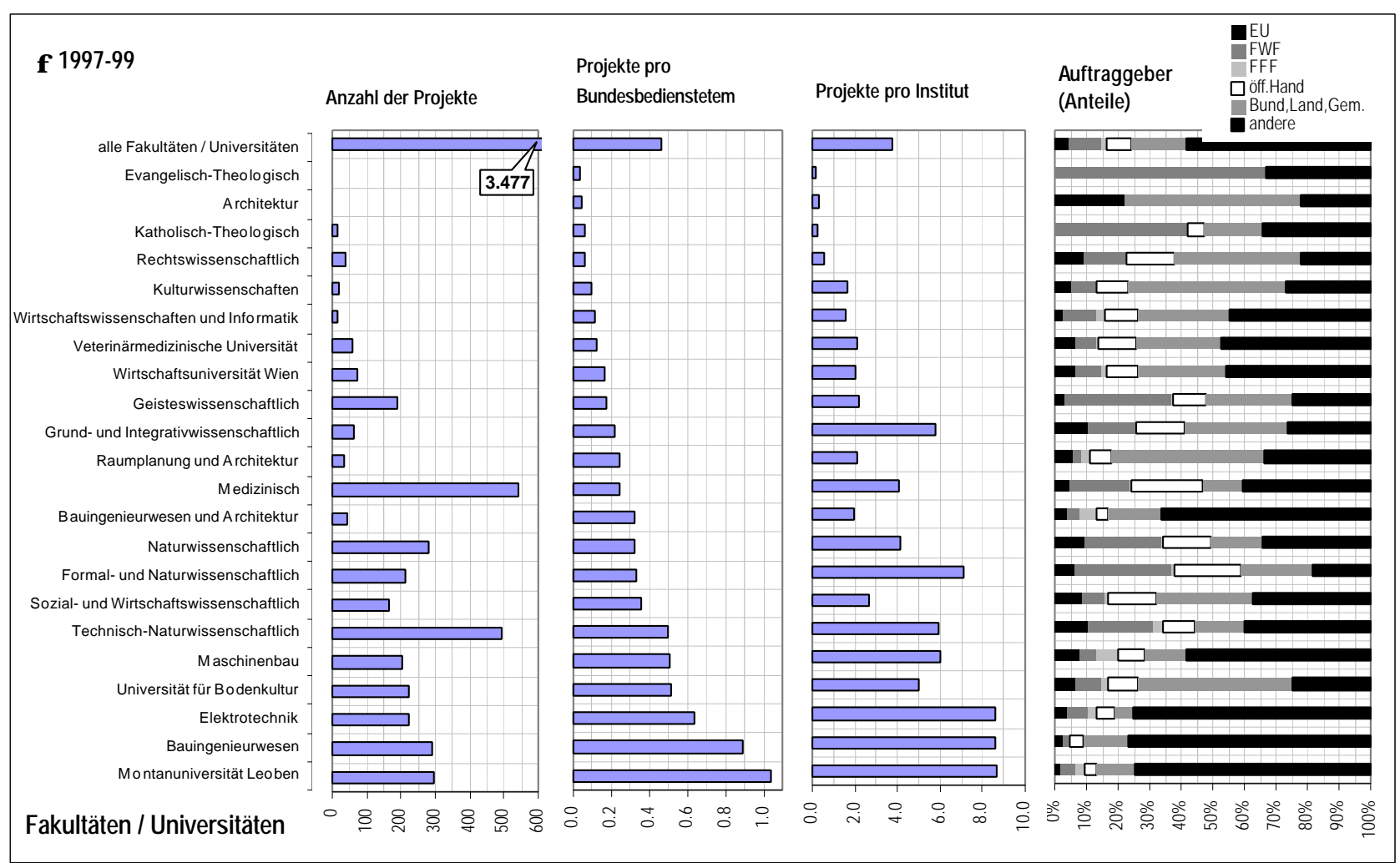

Quelle: BMBWK, Berechnungen von tip

Im Durchschnitt der Jahre 1997-99 wurden insgesamt 3.477 Projekte abgewickelt; die größten Anteile entfielen davon auf die medizinische und technisch-naturwissenschaftliche Fakultät (550 bzw. 500 Projekte). Sowohl bei den Projekten pro Institut als auch pro Bundesbedienstetem weisen die Montanuniversität Leoben, das Bauingenieurwesen und die Elektrotechnik die größten Werte auf. Dies ist nicht weiter verwunderlich, da generell Projektarbeit eine Domäne der technischen Fakultäten darstellt. Für die vorgenannten drei Fakultäten stellen auch "andere" (nicht-öffentliche) Auftraggeber die höchsten Anteile in der Projektstruktur (mit etwa drei Viertel aller Projekte).

Die geringe Projektintensität der Rechtswissenschaftlichen Fakultäten und der Wirtschaftsuniversität Wien bestätigen deren bereits festgestellte Position als vorwiegende (und effiziente) "Ausbildungsinstitutionen".

Auffallend ist, dass bei jenen Instituten mit hoher Projektintensität der private Sektor als Auftraggeber den höchsten Anteil aufweist (Montanuniversität Leoben, Bauingenieurwesen, Elektrotechnik). Der FWF ist bei den Katholischen Fakultäten (die allerdings eine sehr geringe Projektintensität aufweisen) sowie den naturwissenschaftlichen und geisteswissenschaftlichen Fakultäten anteilsmäßig am stärksten vertreten. 


\subsection{Internationaler Vergleich des Publikationsoutputs}

Abbildung 8-12: Entwicklung der Länderanteile an den SCI-Publikationen, 1981-1999

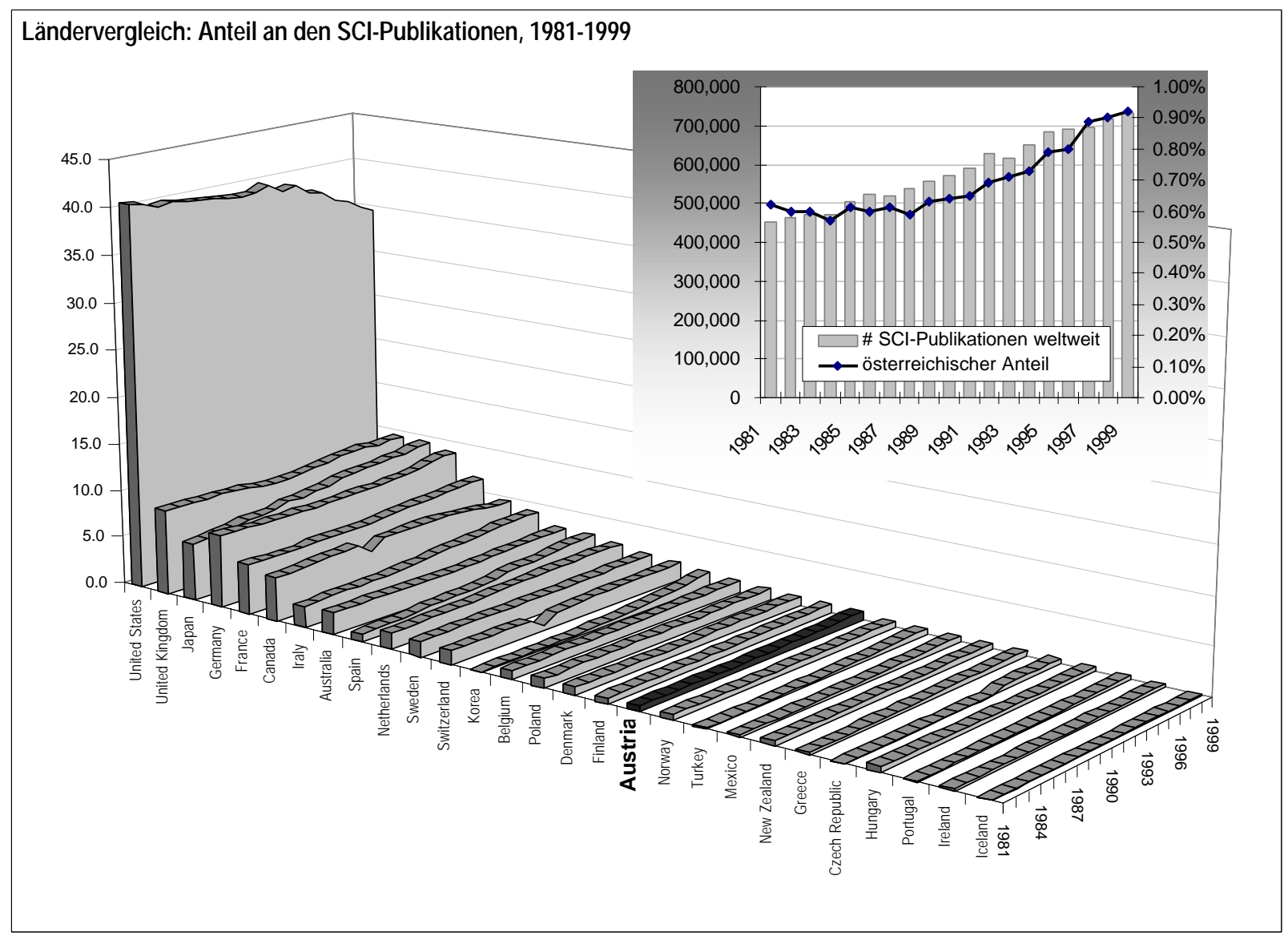

Quelle: Institute for Scientific Information, NSIOD 1981-1999, zit. nach The State and Quality of Scientific Research in Finland, 2000; Berechnungen von tip

Basis dieser Analyse sind die "National Science Indicators" (NSIOD 1981-1999) des Institute for Scientific Information. Dieses Datenbank enthält für den Zeitraum 1981-1999 Angaben zu Veröffentlichung und Zitation in etwa 5.500 naturwissenschaftlichen/technischen, 1.800 sozialwissenschaftlichen und etwa 1.200 geisteswissenschaftlichen Zeitschriften. Die nationale Zuordnung erfolgt durch die Angabe der jeweiligen Instituts/Arbeitsadresse des Autors/Co-Autors bzw. der Autorin/Co-Autorin.

Die obige Grafik zeigt die zeitliche Entwicklung der Länderanteile an der Gesamtzahl der Publikationen (die Länder sind nach ihrem relativen Anteil im Jahr 1999 geordnet). Der Anteil der Publikationen von österreichischen AutorInnen stieg im Beobachtungszeitraum von 0.62 auf $0.92 \%$ und betrug 6.718 Publikationen im Jahr 1999; dies entspricht einer Zunahme des relativen Anteils von etwa $48 \%$. Zum Vergleich: die entsprechenden Zahlen betragen für Deutschland $+15 \%$ (von 7.61 auf $8.79 \%$ ), Schweden $+31 \%$ (von 1.54 auf $2.01 \%$ ), Finnland $+64 \%$ (von 0.58 auf $0.95 \%$ ), Schweiz $+36 \%$ (von 1.38 auf $1.88 \%)$

Festzuhalten bleibt, dass der österreichische Anteil über die 80er Jahre relativ konstant blieb, um dann in den 90er Jahren anzusteigen. Da über den gesamten Zeitraum eine weltweite Zunahme an SCI-Publikationen zu beobachten ist, konnte das österreichische Wissenschaftssystem also nicht nur mit dem allgemeinen Wachstum mithalten sondern sogar seine Position verbessern. 
Abbildung 8-13: Publikationen bezogen auf BIP, Forschungsausgaben und Bevölkerung

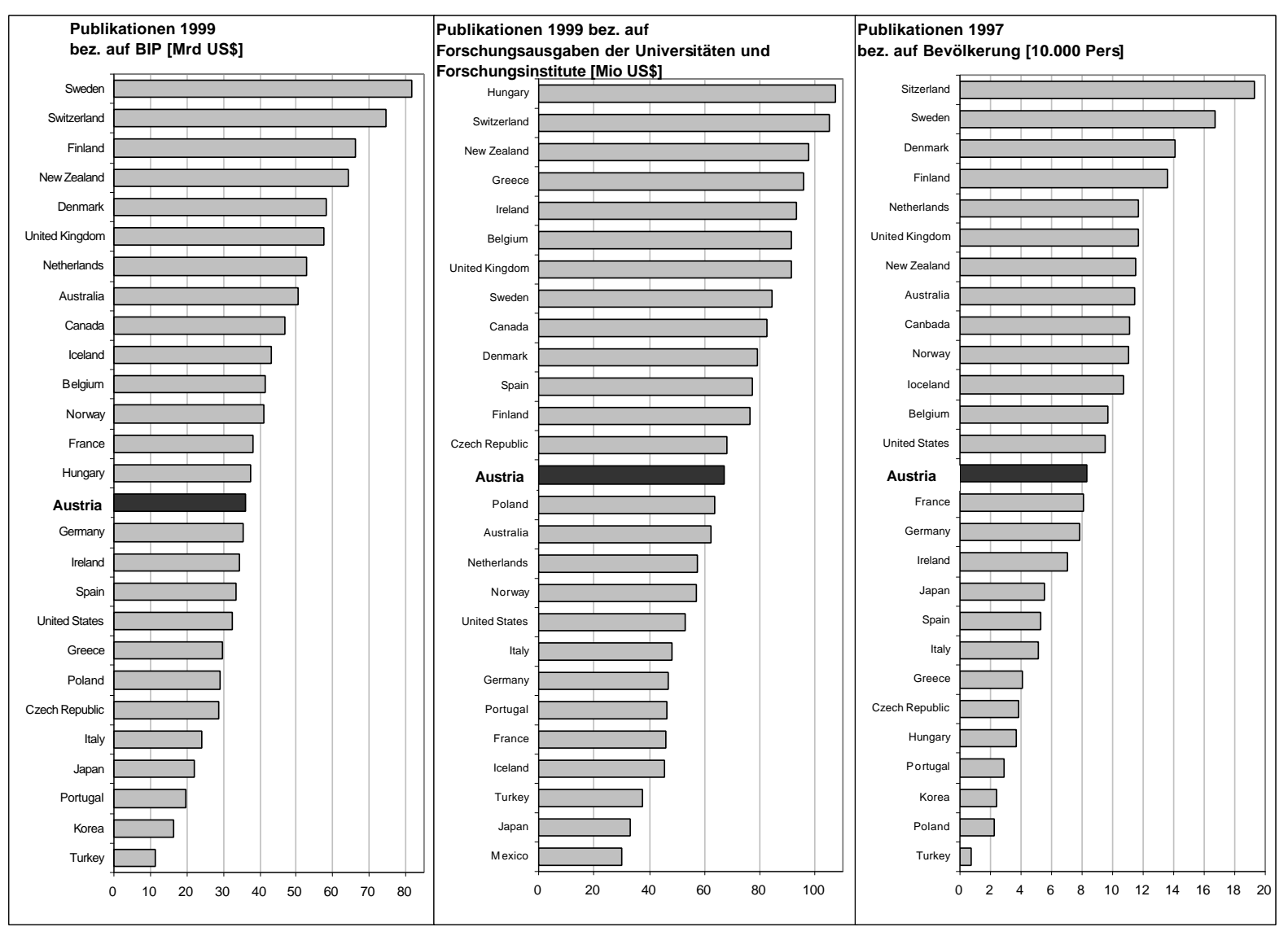

Quelle: Institute for Scientific Information, NSIOD 1981-1999, zit. nach The State and Quality of Scientific Research in Finland, 2000; Berechnungen von tip

Publikationen stellen nur einen Teil des wissenschaftlichen Outputs dar und sind als alleiniger Indikator wenig aussagekräftig. Eine, wenn auch beschränkte, Möglichkeit Ländervergleiche anzustellen, besteht daher darin, Publikationen mit anderen relevanten Indikatoren in Beziehung zu setzen. Die obige Abbildung bezieht die Anzahl der Publikationen auf Indikatoren wie das Bruttoinlandsprodukt, die Forschungsausgaben der Universitäten/Forschungseinrichtungen und die Bevölkerung.

Bei allen drei Indikatoren liegt Österreich jeweils im Mittelfeld, jeweils (knapp) vor Deutschland und (meist deutlich) hinter Schweden, Finnland und der Schweiz, die jeweils meist Spitzenplätze belegen. Interessant ist, dass die in absoluten Zahlen überragenden USA in diesen relativen Rangordnungen nur im (unteren) Mittelfeld aufscheinen. 
Abbildung 8-14: Ländervergleich der Publikationsstruktur nach Wissenschaftsbereichen

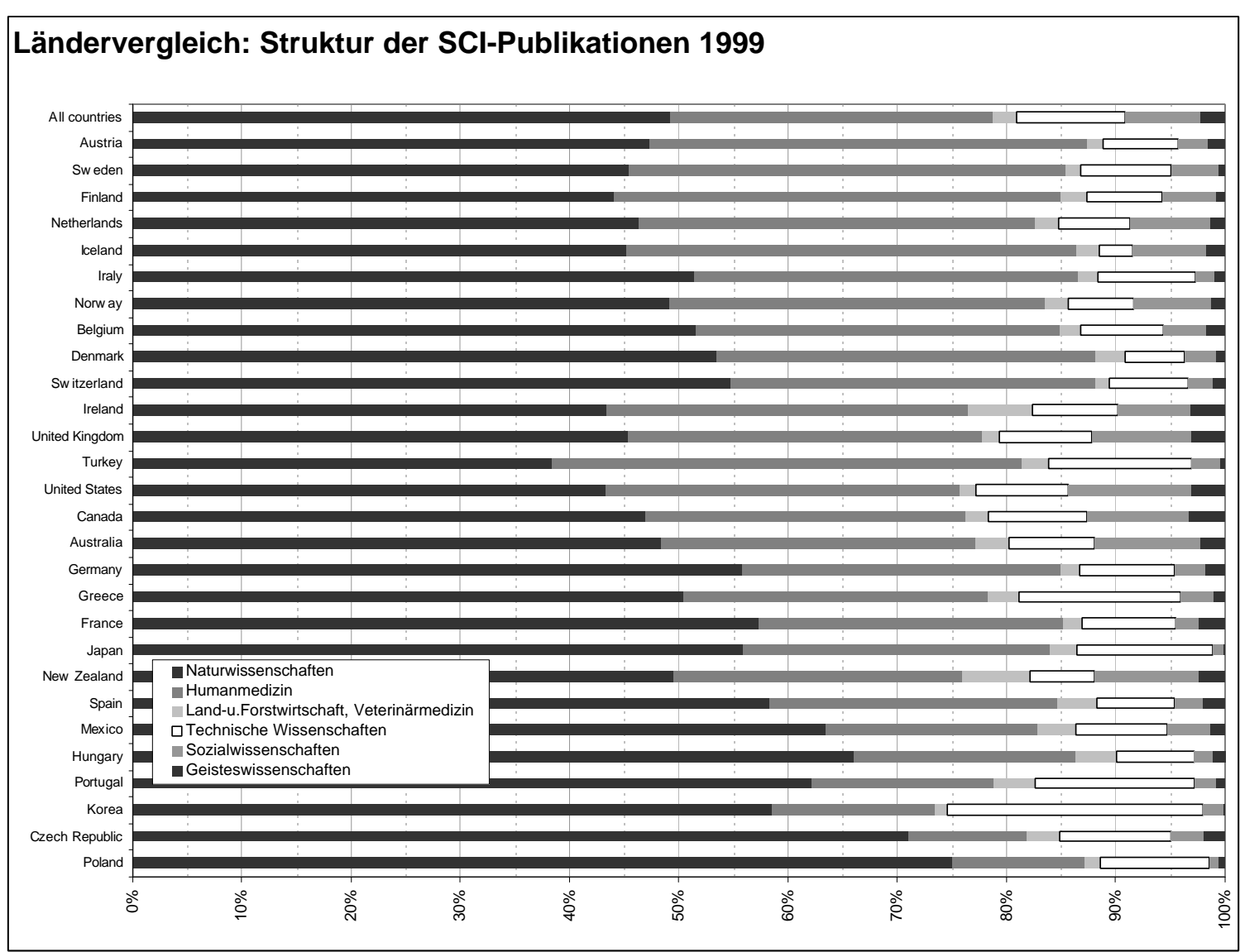

Quelle: Institute for Scientific Information, NSIOD 1981-1999, zit. nach The State and Quality of Scientific Research in Finland, 2000; Berechnungen von tip

Die Grafik zeigt die Struktur der Publikationen, d.h. die Anteile an der Gesamtanzahl, die auf die Wissenschaftsbereiche ${ }^{44}$ entfallen. Die Länder sind nach der "Strukturähnlichkeit" mit Österreich gereiht ${ }^{45}$. Die ähnlichste Publikationsstruktur weisen demnach Schweden und Finnland auf; grob können drei etwa gleich große Ländergruppen nach fallender Strukturähnlichkeit unterschieden werden: kleine europäische Länder; große bzw. englischsprachige Länder; sowie der "Rest" (Mexiko, Korea, osteuropäische Länder). Bei der Humanmedizin weist Österreich mit $40 \%$ den fünfthöchsten Anteil aller 28 Länder auf (die entsprechenden Plätze betragen: 19. bei den Naturwissenschaften, 25. bei der Landund Forstwirtschaft, 22. bei den technischen Wissenschaften, 18. bei den Sozialwissenschaften sowie 13. bei den Geisteswissenschaften).

\footnotetext{
${ }^{44}$ Naturwissenschaften, Humanmedizin, Land- und Forstwirtschaft inkl. Veterinärmedizin, Technische Wissenschaften, Sozialwissenschaften sowie Geisteswissenschaften

${ }^{45}$ Für die Strukturähnlichkeit wurde ein quadratisches Distanzmaß gewählt; es misst den durchschnittlichen Unterschied in den Anteilen der Wissenschaftsbereiche an der Gesamtzahl der Publikationen.
} 
Abbildung 8-15: Impact Factors nach Ländern und Wissenschaftsbereichen

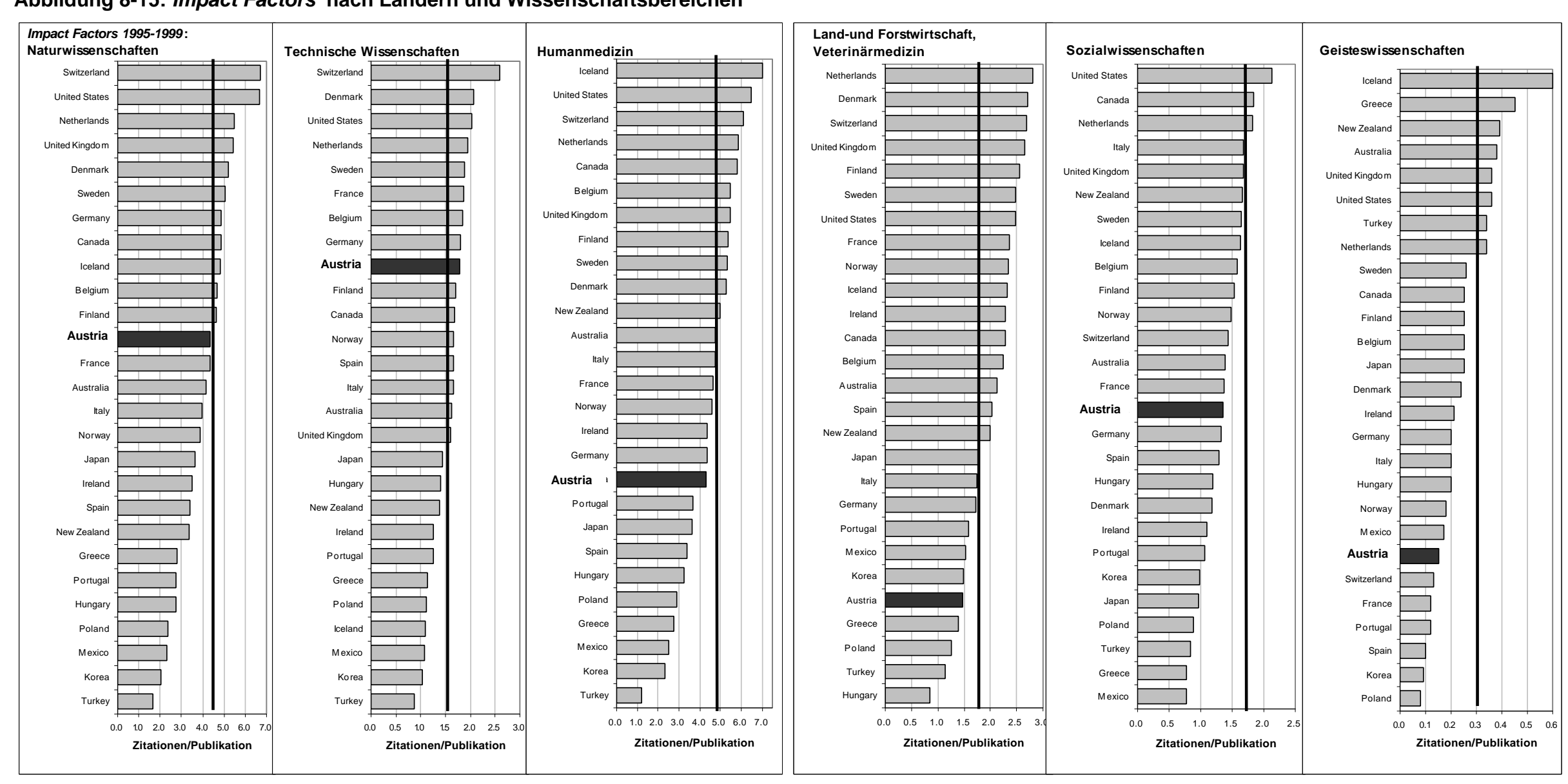

Quelle: Institute for Scientific Information, NSIOD 1981-1999, zit. nach The State and Quality of Scientific Research in Finland, 2000; Berechnungen von tip 
Die Abbildung 8-15 zeigt für die 6 Wissenschaftsbereiche ${ }^{46}$ die Impact Faktoren; diese errechnen sich als Anzahl der Zitationen, bezogen auf die Anzahl der Publikationen (d.h., wie oft ein Artikel im Schnitt zitiert wird); als Bezugslinie ist jeweils der Durchschnitt über alle Länder eingezeichnet.

Obwohl in der Struktur der österreichischen Publikationstätigkeit die Technischen Wissenschaften unterrepräsentiert sind (vergleiche oben), weisen sie den höchsten relativen Impactfaktor aller Wissenschaftszweige auf (etwa $9 \%$ über dem Länderdurchschnitt). Der relativ größte Wissenschaftszweig in Österreich, die Humanmedizin, erreicht nur einen etwas unterdurchschnittlichen Impactfaktor (etwa $10 \%$ unter dem Länderdurchschnitt). Leicht überdurchschnittlich $(+1 \%)$ ist außerdem noch der Impact der naturwissenschaftlichen Publikationen. Im unteren Mittelfeld, jedenfalls mit deutlich unterdurchschnittlichen Impactfaktoren, befinden sich die übrigen Wissenschaftszweige.

Die Vergleichsländer Schweiz, Schweden, Finnland und Deutschland können in den meisten Fällen (z.T. deutlich) höhere Werte verbuchen (die Schweiz ist bei den Natur- und technischen Wissenschaften, der Humanmedizin sowie der Land- und Forstwirtschaft jeweils unter den 3 besten Ländern, nur bei den Geisteswissenschaften liegt die Schweiz als 23. noch 2 Plätze schlechter als Österreich); Finnland liegt in den Natur- und technischen Wissenschaften etwa gleichauf mit Österreich, weist aber in den übrigen Wissenschaftszweigen z.T. deutlich bessere Platzierungen auf. Am ähnlichsten bei den relativen Impactfaktoren stellt sich Deutschland dar.

\footnotetext{
${ }^{46}$ Naturwissenschaften, Technische Wissenschaften, Humanmedizin, Land- und Forstwirtschaft / Veterinärmedizin, Sozialwissenschaften, Geisteswissenschaften
} 


\subsection{Kooperationen zwischen Universitäten und Industrie in Österreich}

Tabelle 8-5: Das Ausmaß von Beziehungen zwischen Universitäten und dem Unternehmenssektor in verschiedenen Interaktionsformen

\begin{tabular}{|c|c|c|c|c|c|c|c|c|}
\hline & $\begin{array}{c}\text { Anteil der } \\
\text { Institute }\end{array}$ & Naturw. & $\begin{array}{c}\text { Technische } \\
\text { W. }\end{array}$ & $\begin{array}{l}\text { Human- } \\
\text { medizin }\end{array}$ & Agrarw. & Sozialw. & Geistesw. & Summe \\
\hline $\begin{array}{l}\text { Anteil am Personal } \\
\text { Interaktionsformen: }\end{array}$ & & 23 & 11 & 31 & 3 & 19 & 12 & 100 \\
\hline Betreuung Dipl./Diss. & 16 & 17 & 31 & 8 & 2 & 42 & 1 & 100 \\
\hline Lehrveranstaltungen & 14 & 7 & 20 & 4 & 1 & 60 & 8 & 100 \\
\hline Drittmittelpersonal & 13 & 28 & 39 & 14 & 7 & 10 & 2 & 100 \\
\hline Forschermobilität & 13 & 37 & 34 & 14 & 1 & 12 & 1 & 100 \\
\hline Gem. Projekte & 12 & 21 & 45 & 17 & 3 & 13 & 0 & 100 \\
\hline Weiterbildung & 11 & 6 & 24 & 15 & 1 & 46 & 8 & 100 \\
\hline Gem. Publikationen & 11 & 28 & 38 & 14 & 5 & 14 & 0 & 100 \\
\hline Neugründungen & 6 & 29 & 31 & 7 & 1 & 30 & 1 & 100 \\
\hline Sabbaticals & 4 & 24 & 29 & 10 & 7 & 29 & 2 & 100 \\
\hline Total & 23 & 29 & 29 & 23 & 18 & 23 & 13 & \\
\hline
\end{tabular}

Quelle: Institutsbefragung 1999 an den 12 Universitäten, siehe Schartinger et al. 2000. Angaben in Prozent.

Tabelle 8-5 gibt einen Überblick über verschiedene Formen von wissensintensiven Interaktionen zwischen Universitäten und dem Unternehmenssektor in Österreich. In einer Vollerhebung im Jahr 1999 wurden die Universitätsinstitute der 12 Universitäten über das Ausmaß ihrer Beziehungen zum Unternehmenssektor in folgenden Interaktionsformen befragt:

- Gemeinsame Betreuung von Diplomarbeiten und Dissertationen mit dem Unternehmenssektor;

- Lehrveranstaltungen, die von Angehörigen des Unternehmenssektors an Universitätsinstituten gehalten werden;

- Personal, das durch Drittmittel von Unternehmen bezahlt wird;

- Wechsel von Universitätsassistenten in den Unternehmenssektor;

- gemeinsame Projekte mit dem Unternehmenssektor;

- Weiterbildung von Universitätsinstituten für Angehörige des Unternehmenssektors;

- gemeinsame Publikationen mit dem Unternehmenssektor;

- Neugründungen von Firmen aus Universitäten; Sabbaticals von Universitätsforschern im Unternehmenssektor.

Aus der Befragung geht hervor, dass zumindest $23 \%$ aller Universitätsinstitute im Zeitraum 1995-98 über wenigstens eine der genannten Interaktionsformen einmal Kontakt zum Unternehmenssektor hatten. Der entsprechende Anteil der Institute pro Wissenschaftszweig variiert stark. In den Natur- und Technischen Wissenschaften beträgt der Anteil der Institute mit Kontakten zum Unternehmenssektor in den genannten Formen im Zeitraum 1995-98 zumindest knapp $30 \%$, in der Humanmedizin und in den Sozialwissenschaften knapp ein Viertel und in den Agrar- und Geisteswissenschaften unter $20 \%$.

Was die Intensität der Interaktionen betrifft, so zeigt sich, dass etwa die Technischen Wissenschaften, die über $11 \%$ des Universitätspersonals verfügen, $31 \%$ aller Betreuungsaktivitäten von Diplomarbeiten und Dissertationen gemeinsam mit dem Unternehmenssektor auf sich vereinen. In Relation zu ihrer Größe weisen sie damit eine überdurchschnittliche Interaktionsintensität in der Interaktionsform "gemeinsame Betreuung wissenschaftlicher Arbeiten“ auf, sowie in allen anderen Interaktionsformen. Die besondere Präferenz dieses Wissenschaftszweiges (besonders hoch ist ihre Interaktionsintensität) liegt jedoch in der Interaktionsform „gemeinsame Projekte“. 
Auch die Bereiche Naturwissenschaften und Agrarwissenschaften weisen in einigen Interaktionsformen überdurchschnittliche Interaktionsintensitäten auf. Dabei erfolgt in den Naturwissenschaften ein besonders intensiver Wissensfluss in Form der Mobilität von Universitätsforschern in den Unternehmenssektor, in den Agrarwissenschaften hingegen über die gemeinsame Betreuung von Vertragsassistenten, die aus Drittmitteln vom Unternehmenssektor finanziert werden.

Tabelle 8-6: Unternehmenssektoren und ihre Beziehungen zu Universitäten in verschiedenen Interaktionsformen

\begin{tabular}{|c|c|c|c|c|c|c|c|}
\hline Wirtschaftszweig & Nace & $\begin{array}{c}\text { FuE- } \\
\text { Quote }^{47}\end{array}$ & $\begin{array}{l}\text { Gem. Pub- } \\
\text { lizieren }\end{array}$ & $\begin{array}{c}\text { Gem. Pro- } \\
\text { jekte }\end{array}$ & $\begin{array}{l}\text { Mobilität v. } \\
\text { Univ. in Ind. }\end{array}$ & Spin-offs & $\begin{array}{c}\text { Gem. Aus- } \\
\text { bildung }\end{array}$ \\
\hline \multicolumn{8}{|l|}{ Produktionssektor } \\
\hline Ernährungsindustrie & (nace 15-16) & 0,56 & 0,82 & 0,27 & 1,42 & 0,00 & 1,09 \\
\hline Textilindustrie & (nace 17-19) & 2,37 & 0,00 & 0,48 & 0,00 & 0,00 & 0,03 \\
\hline Holzindustrie & (nace 20) & 0,80 & 0,00 & 0,00 & 0,42 & 0,00 & 1,24 \\
\hline Papierindustrie & (nace 21-22) & 0,64 & 1,22 & 0,63 & 1,98 & 0,00 & 4,73 \\
\hline Chemische Industrie & (nace 23-24) & 12,66 & 2,38 & 3,69 & 2,66 & 0,22 & 1,68 \\
\hline Kunststoffindustrie & (nace 25) & 1,61 & 0,99 & 25,60 & 1,25 & 0,00 & 0,59 \\
\hline Glasindustrie & (nace 26) & 2,77 & 0,36 & 3,55 & 0,00 & 0,00 & 0,21 \\
\hline Metallindustrie & (nace 27-28) & 1,53 & 2,58 & 3,98 & 0,81 & 0,00 & 1,49 \\
\hline Maschinenbau & (nace 29) & 3,91 & 0,95 & 1,17 & 0,92 & 0,43 & 0,66 \\
\hline Elektro(nik)industrie & (nace $30-32$ ) & 16,71 & 0,37 & 0,40 & 0,17 & 0,06 & 0,22 \\
\hline Medizintechnik & (nace 33) & 6,41 & 5,75 & 1,27 & 3,37 & 3,81 & 2,23 \\
\hline Kfz-Industrie & (nace $34-35,50)$ & 2,79 & 1,03 & 1,20 & 0,65 & 0,00 & 2,35 \\
\hline Möbelindustrie & (nace 36) & 1,0 & 0,91 & 0,22 & 0,00 & 0,00 & 1,23 \\
\hline Abfallverwertung & (nace 37,90 ) & 0,85 & 5,39 & 1,24 & 1,94 & 0,00 & 3,64 \\
\hline \multicolumn{8}{|l|}{ Dienstleistungssektor } \\
\hline Handel & (nace 51-52) & 0,02 & 5,39 & 0,43 & 4,25 & 0,00 & 2,22 \\
\hline TransportDL & (nace 60-63) & 0,0 & 21,57 & 10,83 & 27,21 & 0,00 & 65,05 \\
\hline Telekommunikation & (nace 64) & 0,09 & 2,59 & 1,39 & 10,88 & 0,00 & 2,68 \\
\hline FinanzDL & (nace 65-67) & 0,08 & 6,47 & 1,19 & 4,42 & 0,00 & 9,57 \\
\hline SoftwareDL & (nace 72) & 3,88 & 0,86 & 0,17 & 4,06 & 5,33 & 1,05 \\
\hline$F \& E$ & (nace 73) & 56,75 & 2,58 & 0,47 & 1,76 & 0,54 & 0,83 \\
\hline UnternehmensDL & (nace 74) & 0,14 & 23,73 & 2,82 & 43,20 & 199,70 & 50,64 \\
\hline
\end{tabular}

Quelle: Institutsbefragung 1999 an den 12 Universitäten, siehe Schartinger et al. 2000.

Die Tabelle 8-6 versucht diejenigen Unternehmenssektoren zu identifizieren, die besonders starke Verbindungen zu Universitäten aufweisen. Hierzu wird die Anzahl der Interaktionen eines Unternehmenssektors mit der „zu erwartenden Interaktionsintensität“ aufgrund der Größe des Sektors verglichen. ${ }^{48}$ Zur besseren Anschaulichkeit ist weiters in der linken Spalte die FuE-Intensität für jeden Sektor ausgewiesen. (Die verschiedenen Interaktionsformen wurden teilweise aggregiert: Die Interaktionsform "Gemeinsame Ausbildung" umfasst die gemeinsame Betreuung von Diplomarbeiten und Dissertationen mit dem Unternehmenssektor; Lehrveranstaltungen, die von Angehörigen des Unternehmenssektors an Universitätsinstituten gehalten werden und die Weiterbildung auf Universitätsinstituten für Angehörige des Unternehmenssektors. Die Interaktionsform "Mobilität“ umfasst gleichzeitig Sabbaticals und den dauerhaften Wechsel von Universitätsforschern in die Industrie.)

Im produzierenden Sektor zeigt sich, dass nicht notwendigerweise die FuE-intensivsten Unternehmenssektoren auch eine besonders hohe Interaktionsintensität in allen Interaktionsformen zeigen: Die Elektronikindustrie weist in allen Interaktionsformen eine bedeutend niedrigere Interaktionsintensität auf, als aufgrund ihrer Größe und ihrer FuE-Intensität zu erwarten wäre. Anders in der Medizintechnik: Dieser Unternehmenssektor weist im Vergleich zu seiner Größe in allen genannten Interaktionsformen eine überdurchschnittlich hohe Interaktionsintensität auf. Weiters zeigen einzelne Unternehmenssek-

\footnotetext{
${ }^{47}$ Anteil der FuE-Beschäftigten an den gesamten Beschäftigten

${ }^{48}\left(\mathrm{I}_{\mathrm{s}} / \Sigma \mathrm{I}_{\mathrm{s}}\right) /\left(\mathrm{S}_{\mathrm{s}} / \Sigma \mathrm{S}_{\mathrm{s}}\right)$, wobei $\mathrm{I}_{\mathrm{s}}$ für die Anzahl der Interaktionen eines Unternehmenssektors s steht und $\mathrm{S}_{\mathrm{s}}$ für die Anzahl von FuEAngestellten dieses Unternehmenssektors.
} 
toren deutliche Präferenzen für bestimmte Interaktionstypen: So halten die Sektoren Maschinenbau und Glasindustrie hauptsächlich über gemeinsame Projekte Beziehungen zu österreichischen Universitäten aufrecht, die Ernährungsindustrie hingegen über die Mobilität von Universitätsforschern und über gemeinsame Ausbildungsaktivitäten.

Im Dienstleistungssektor weist der Unternehmenssektor "Forschung und Entwicklung", der definitionsgemäß auch der FuE-intensivste Sektor ist, v.a. über gemeinsame Publikationen und die Mobilität von Universitätsforschern überdurchschnittlich intensive Kontakte zu Universitäten auf. Der Sektor „Softwaredienstleistungen" zeigt deutliche Präferenz für Mobilität, Spin-offs und gemeinsame Ausbildungsaktivitäten mit Universitäten. Insgesamt sind es drei Unternehmenssektoren, die eine höhere Intensität an Unternehmensneugründungen aus Universitäten heraus aufweisen, als aufgrund ihrer Größe zu erwarten wäre: Medizintechnik, Softwaredienstleistungen und v.a. der unternehmensnahe Dienstleistungssektor.

Tabelle 8-7: Unternehmenssektoren und ihre extramuralen FuE-Ausgaben für Universitäten

\begin{tabular}{|c|c|c|c|c|c|}
\hline Wirtschaftszweig & Nace & $\begin{array}{c}\text { EFuE_Univ/ } \\
\text { BWS }^{1} \\
\text { (A) }\end{array}$ & $\begin{array}{c}\text { EFuE_gesamt/ } \\
\text { BWS }^{2} \\
\text { (B) }\end{array}$ & $\begin{array}{c}A / B^{3} \\
\text { (C) }\end{array}$ & 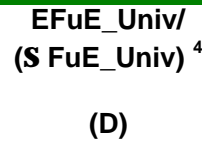 \\
\hline Forschung und Entwicklung & 73 & 0,82 & 5,36 & 15,30 & 2,27 \\
\hline Medizintechnik & 33 & 0,12 & 1,32 & 9,09 & 3,93 \\
\hline Elektro(nik)ind. & 31 & 0,08 & 0,63 & 12,70 & 5,85 \\
\hline Chemische Ind. & 24 & 0,07 & 1,23 & 5,69 & 7,45 \\
\hline Glasind. & 26 & 0,07 & 0,29 & 24,14 & 6,64 \\
\hline Energie/Wasserversorgung & $40-41$ & 0,05 & 0,13 & 38,46 & 11,32 \\
\hline Maschinenbauind. & 29 & 0,05 & 0,55 & 9,09 & 8,52 \\
\hline Holzind. & 20 & 0,04 & 0,10 & 40,00 & 3,17 \\
\hline Datenverarbeitung & 72 & 0,03 & 0,18 & 16,67 & 1,87 \\
\hline Kunststoffind. & 25 & 0,03 & 4,58 & 0,66 & 1,95 \\
\hline Rundfunk; TV; Nachrichtentechnik & 32 & 0,02 & 2,81 & 0,71 & 2,54 \\
\hline Bankdienstl. & $65-67$ & 0,02 & 0,15 & 13,33 & 10,58 \\
\hline Metallind. & $27-28$ & 0,02 & 0,22 & 9,09 & 5,08 \\
\hline Nahrungs-und Genussmittelind. & 15 & 0,02 & 0,12 & 16,67 & 2,77 \\
\hline UnternehmensDL; Real.; Vermiet. & $70-71,74$ & 0,02 & 0,08 & 25,00 & 7,73 \\
\hline Verkehr/ Nachrichtenübermittlung & $60-64$ & 0,01 & 0,08 & 12,50 & 6,45 \\
\hline Schmuck etc. & 36 & 0,01 & 0,12 & 8,33 & 0,93 \\
\hline Textilind. & 17 & 0,01 & 0,43 & 2,33 & 0,30 \\
\hline Papierind.; Druckereiwesen & $21-22$ & 0,01 & 0,23 & 4,35 & 0,85 \\
\hline Handel & $50-52$ & 0,01 & 0,02 & 50,00 & 5,63 \\
\hline Fahrzeugbau & 34 & 0,00 & 3,95 & 0,00 & 0,37 \\
\hline Bauind. & 45 & 0,00 & 0,01 & 0,00 & 0,02 \\
\hline Recycling & 37 & 0,00 & 0,00 & - & 0,00 \\
\hline Tourismus & 55 & 0,00 & 0,00 & - & 0,00 \\
\hline
\end{tabular}

Quelle: Statistik Österreich; FuE-Erhebung 1998. Angaben in Prozent.

\footnotetext{
${ }^{1}$ Extramurale FuE-Ausgaben für Universitäten in Prozent der Bruttowertschöpfung

${ }^{2}$ Extramurale FuE Ausgaben gesamt in Prozent der Bruttowertschöpfung

${ }_{3}^{3}$ Anteil der extramuralen FuE-Ausgaben für Universitäten an den gesamten extramuralen FuE-Ausgaben eines Sektors

${ }^{4}$ Extramurale FuE-Ausgaben für Universitäten pro Sektor in Prozent der gesamten extramuralen FuE- Ausgaben für Universitäten über alle Sektoren
}

Tabelle 8-7 zeigt die extramuralen FuE-Ausgaben der einzelnen Unternehmenssektoren für Universitäten. Der Sektor Forschung und Entwicklung weist dabei mit 0,82 \% der Bruttowertschöpfung die relativ höchsten Anteile an extramuralen FuE-Ausgaben für Universitäten auf. Mit deutlichem Abstand folgen die Sektoren Medizintechnik, die Elektronik- und die Chemische Industrie. 
Es gibt Unternehmenssektoren, die mehr als alle anderen dazu tendieren, externes Wissen einzukaufen und für Innovationen im technologischen oder organisatorischen Bereich zu verwenden. $\mathrm{Zu}$ diesen Sektoren, die allgemein über einen hohen Anteil an extramuralen FuE-Ausgaben an der Bruttowertschöpfung verfügen (Tabelle 8-7, Spalte B), gehören der FuE-Sektor, die Kunststoffindustrie, der Fahrzeugbau und der Sektor Rundfunk-, TV- und Nachrichtentechnik. Als Quellen für relevantes externes Wissen kommen außer Universitäten auch andere Einrichtungen in Frage, wie etwa andere Unternehmen und sonstige staatliche oder private Einrichtungen. Ein Vergleich der Spalten A und B zeigt, dass dabei von den hier genannten Unternehmenssektoren, die in hohem Maße externes Wissen zukaufen, nur der Sektor Forschung und Entwicklung in nennenswertem Ausmaß auf das Wissen von Universitäten zurückgreift (15,3\% der gesamten extramuralen FuE-Ausgaben des Sektors, Spalte C).

Die Sektoren hingegen, die die höchsten Anteile ihrer extramuralen FuE-Ausgaben an Universitäten vergeben (Spalte C), sind der Handel (50\%), die Holzindustrie (40), Energie- und Wasserversorgung (38,5\%), der unternehmensnahe Dienstleistungssektor (25\%) und die Glasindustrie (24\%).

Spalte D weist für jeden Sektor den Anteil seiner extramuralen FuE-Ausgaben für Universitäten an der Summe aller extramuralen FuE-Ausgaben für Universitäten aus. Dabei zeigt sich, dass Universitäten mengenmäßig den höchsten Betrag an Drittmitteln aus dem Unternehmenssektor Energie- und Wasserversorgung (11,3\%) bekommen, gefolgt von Bankdienstleistungen (10,6\%) und der Maschinenbauindustrie $(8,5 \%)$. 


\section{Literatur}

Almus, M., Egeln, J., Engel, D., Gassler, H. (2000): Unternehmensgründungsgeschehen in Österreich bis 1998, ZEW Dokumentation, (00-06), Mannheim.

Dachs B., Leo H. (1999): Innovationsaktivitäten der österreichischen Wirtschaft: Band 2: Dienstleistungssektor, WIFO, Wien.

Dachs, B., Knoll. (2001): Screening eEurope. Technologiepolitik für die Informationsgesellschaft. Studie im Rahmen des TIP, Wien.

DIW (Hrsg.) (2000): Die Ökonomie der Informationsgesellschaft, Vierteljahresheft 4/2000, Deutsches Institut für Wirtschaftsforschung, Berlin.

DoC (U.S. Department of Commerce) (1999): The Emerging Digital Economy II, Washington, D.C.

DoC (U.S. Department of Commerce) (2001): Digital Economy 2000, Washington http://www.esa.doc.gov/de2k2.htm.

EITO (2001): European Information Technology Observatory 2001, Frankfurt am Main.

Eliasson, G., Fölster, S., Lindberg, T., Pousette, T., Taymaz, E. (1990): The Knowledge-based Information Economy, Almquist \& Wicksell International, Stockholm.

"Erklärung der Bundesregierung zu aktuellen Fragen der Forschungs- und Technologiepolitik", beschlossen im Ministerrat am 11. Juli 2000, Wien.

Europäische Kommission (1994): Europe's Way to the Information Society: An Action Plan, KOM (94)347 final, Brussels.

Europäische Kommission (2000): eEurope 2002: Eine Informationsgesellschaft für alle, Aktionsplan, vorgelegt am Gipfel von Lissabon, 23/24. März 2000.

Gassler, H., Polt, W., Rammer, C. (1999): Erhöhung der österreichischen F\&E Quote bis 2005: Modellrechnungen, Studie im Auftrag des BMWA, OEFZS--S-0032. Seibersdorf.

Geyer, A., Rammer, C, Pointner, W, Polt, W., Hollenstein, Donzé, L., W, Arvanitis, S., (2000): Evaluierung des ITF-Schwerpunktes FlexCIM, ARCS Report OEFZS-S-0102, Seibersdorf.

Guellec, D., van Pottelsberghe, B. (2000): The Impact of Public R\&D Expenditure on Business R\&D, STI Working Papers, 2000 (4). Paris

Hall, B., van Reenen, J., (2000): How Effective are Fiscal Incentives for R\&D? A Review of the Evidence, in: Research Policy, 29(4-5), S. 449-469.

Harhoff, D. (1994): Zur steuerlichen Behandlung von Forschungs- und Entwicklungsaufwendungen. Eine internationale Bestandsaufnahme, ZEW Dokumentation (94-02), Mannheim.

Hipp C. (2000): Innovationsprozesse im Dienstleistungssektor., Physica, Heidelberg.

Hutschenreiter G., Kaniovski S. (1999): Embodied Technology Diffusion in the Austrian Economy. Studie im Rahmen des TIP, Wien.

Hutschenreiter, G. (Koordination), Knoll, N., Paier, M., Ohler, F. (1998): Österreichischer Technologiebericht 1997, tip-Programm, Wien.

Hutschenreiter, G. (1993): Neue Ansätze zu einer Industriepolitik der EG, in: WIFO-Monatsberichte, 66(5).

Hutschenreiter, G. (1995): Intersektorale und internationale "F\&E-Spillovers". in: Externe Effekte von Forschung und Entwicklung, in: WIFO-Monatsberichte, 68(6), S. 419-427.

Hutschenreiter, G. (1998): Produktivität und Technologiediffusion, in: Wirtschaftspolitische Blätter, 45(1), S. 28-37.

Hutschenreiter, G., Kaniovski, S. (1999): Embodied Technology Flows in the Austrian Economy, Studie im Auftrag der Bundesministerien für wirtschaftliche Angelegenheiten sowie für Wissenschaft und Verkehr, Technologie - Information - Politikberatung (TIP), Wien. 
Hutschenreiter, G., Leo, H. (1998): Technologiepolitik und -förderung, in: Bayer, K. (Koordination), Der Staat und seine Funktionen. Neue Formen der Erfüllung öffentlicher Aufgaben, S. 151-175, Bundesministerium für Finanzen, Wien.

Hutschenreiter, G., Peneder, M., „Österreichs Technologielücke im Außenhandel“, in WIFOMonatsberichte, 1997, 70 (2), S. 103-114.

Hutschenreiter, G., Polt, W. Gassler, H. (2001): Möglichkeiten zur Erhöhung der österreichischen Forschungsquote - Abschätzung der Effekte öffentlicher auf private F\&E-Ausgaben. Endbericht an die AG ,Forschungsquote' im Auftrag des BMVIT, Wien.

ITU (1995), World Telecommunication Development Report 1995: Information Indicators, Geneva.

Jörg, L., Pointner, W., Polt, W., Zinöcker, K. (2001): Evaluierung des ITF-Schwerpunktes TechTransfer, Wien.

Leo, H. (1999): Österreichs Innovations- und Forschungsleistung im internationalen Vergleich, in WIFO-Monatsberichte, 72(6), S. 435-443.

Leyden, D.P., Link, A.N. (1992): Government's Role in Innovation, Kluwer Academic Publishers, Dordrecht.

Lundvall, B. (1997): Information Technology in the Learning Economy: Challenges for Development Strategies, Communications \& Strategies 28/4, pp.177-191.

Movery, D. (1995): The Pratice of Technology Policy, in: Stoneman, P. (Hsg.), Handbook of the Economics of Innovation and Technical Change, pp. 513-557, Blackwell, Oxford.

OECD (2001), Communications Outlook 2001, Paris.

OECD (2000a), Science Technology and Industry Outlook 2000, Paris.

OECD (2000b), A New Economy?: The changing role of innovation and information technology in growth, Paris.

OECD (2000c), Measuring the ICT Sector, Paris.

OECD-BSTS (2000): Basic Science and Technology Statistics (BSTS), Paris.

OECD-MSTI (2000): Main Science and Technology Indicators (MSTI), Paris.

OECD (1999): Science, Technology and Industry Scoreboard 1999. Benchmarking Knowledge-Based Economies, Paris.

OECD (1998): Internationalisation of Industrial R\&D. Patterns and Trends, Paris.

OECD (1998)Technology, Productivity and Job Creation - Best Policy Practices, Paris.

OECD (1997), Policy Evaluation in Innovation and Technology. Towards Best Practices, Paris.

OECD (1996), The Knowledge-based Economy, Paris.

Peneder, M. (2001), Entrepreneurial Competition and Industrial Location, Edward Elgar, Cheltenham, UK.

Peneder, M. (1999), The Austrian Paradox: "Old" Structures but High Performance?, in: Austrian Economic Quarterly, 1999 (4), pp. 239-247, Wien.

Polt, W. (Koordination), Gassler, H., Hutschenreiter, G., Knoll, N., Leo, H., Paier, M., Peneder, M., Schibany, A. (1999): Österreichischer Technologiebericht, tip-Programm, Wien.

Polt, W. (2000): Policy case study Austria. Report prepared for the OECD Focus Group on Innovative Firm Network, Wien.

Polt, W., Daniel, H. D., Fritz, O, (2000), Evaluierung von F\&E-Förderungen. Ein internationaler Vergleich am Beispiel Finnland, Wien.

Schartinger, D., Gassler, H., Schibany, A. (2000): Benchmarking Industry - Science Relations, ARCS Report OEFZS-S-0099, Seibersdorf. 
Schibany, A., Jörg, L., Gassler, H., Warta, K., Sturn, D., Polt, W., Streicher, G., Luukkonen, T., Arnold, E. (2001): Evaluierung der österreichischen Beteiligung am 4. Rahmenprogramm der EU für Forschung, technologische Entwicklung und Demonstration, Wien.

Schibany, A. (1998): Co-operative Behaviour of Innovative Firms in Austria, tip Programm, Wien.

Schneider, H.W. (2000): Steuerliche Begünstigung von Forschung und Entwicklung, Linde, Wien.

Scholtze, E. (2000): Finanzierung der Ausgaben für Forschung und experimentelle Entwicklung in Österreich. Globalschätzung 2000. In: Statistische Nachrichten 10/2000, S. 792-796.

Shapiro, C., Varian, H. (1999): Information Rules: A Strategic Guide to the Network Economy, Harvard Business School Press, Boston, Massachusetts.

Statistik Austria (2001): Forschung und experimentelle Entwicklung (F\&E) im firmeneigenen Bereich 1998. In: Statistische Nachrichten 2/2001, S. 89-103.

Statistik Austria (2000a): Finanzierung der Ausgaben für Forschung und experimentelle Entwicklung in Österreich. Globalschätzung 2000. In: Statistische Nachrichten 10/2000, S. 792-796.

Statistik Austria (2000a): Produzierender Bereich 1998, Wien.

Statistik Austria (2000b): Handel, Dienstleistungen 1998, Wien.

Statistik Austria (1998a): Produzierender Bereich 1995, Wien.

Statistik Austria (1998b): Handel, Dienstleistungen 1995, Wien.

Sturn, D., Novacovic, M., Pointner, W., (2000), Unterstützung bei der Konkretisierung und Ausformulierung eines Förderungsprogramms "Akademische Spin-offs", Wien-Graz.

Sundbo, J., Gallouj, F. (1998): Innovation in Services. SI4S Project Synthesis Report. http://www.step.no/Projectarea/si4s/start.htm

Tichy, G. (2001): Das Nutzer-Paradoxon und seine Bedeutung für die europäische Innovationsschwäche. Neue Ansatzpunkte für die Technologiepolitik?, in: Fuchs, W. und Horvath, G. Hg. Wirtschaftsstandort Österreich. Von der Theorie zur Praxis, Wien: Bundesministerium für Wirtschaft und Arbeit, 207-30.

WIFO, Forschungszentrum Seibersdorf, Joanneum Research (1996): Technologiepolitisches Konzept 1996 der Bundesregierung, Expertenentwurf, Wien. 


\section{Anhänge}

I Statistischer Anhang

II Szenarienrechnungen des "Tip" zur Erhöhung der F\&E-Quote

III Arbeitsaufträge aus der Erklärung der Bundesregierung vom 11. Juli 2000

III/1: Bericht der Projektgruppe „Hebung der Forschungsquote in der Wirtschaft“ vom 8. März an die Bundesregierung

III/2: Statistische Erhebungen - Synchronisierung mit internationalen Organisationen

III/3: Erweiterung der Bund/-Bundesländer-Kooperation

III/4: Programm "Public Understanding of Science and Technology" 


\section{STATISTISCHER ANHANG I: TABELLENÜBERSICHT}

\begin{tabular}{|c|c|}
\hline abelle & Ing \\
\hline 1 & $\begin{array}{l}\text { Globalschätzung 2001: Bruttoinlandsausgaben für F\&E. Finanzierung der in Österreich durchge- } \\
\text { führten Forschung und experimentellen Entwicklung } 1981 \text { - } 2001 \text { in Millionen Schilling }\end{array}$ \\
\hline $1 a$ & $\begin{array}{l}\text { Globalschätzung 2001: Bruttoinlandsausgaben für F\&E. Finanzierung der in Österreich durchge- } \\
\text { führten Forschung und experimentellen Entwicklung } 1981 \text { - } 2001 \text { in Prozent des BIP }\end{array}$ \\
\hline 2 & $\begin{array}{l}\text { Ausgaben des Bundes für Forschung und Forschungsförderung } 1998 \text { bis } 2001 \text { nach Ressorts. } \\
\text { Aufgliederung der Beilage T der Amtsbehelfe zu den Bundesfinanzgesetzen } 2000 \text { und } 2001 \text { (Teil a } \\
\text { und Teil b) }\end{array}$ \\
\hline 3 & $\begin{array}{l}\text { Forschungswirksame Ausgaben des Bundes } 1998 \text { bis } 2001 \text { nach Ressorts. Beilage T des Amtsbe- } \\
\text { helfs zum Bundesfinanzgesetz } 2001 \text { (Teil a und b) }\end{array}$ \\
\hline 4 & $\begin{array}{l}\text { Ausgaben des Bundes } 1989 \text { bis } 2001 \text { für Forschung und Forschungsförderung nach sozio- } \\
\text { ökonomischen Zielsetzungen (ÖSTAT-Systematik) }\end{array}$ \\
\hline 5 & $\begin{array}{l}\text { Ausgaben des Bundes } 1998 \text { für Forschung und Forschungsförderung nach sozio-ökonomischen } \\
\text { Zielsetzungen (ÖSTAT-Systematik) und Ressorts }\end{array}$ \\
\hline 6 & $\begin{array}{l}\text { Ausgaben des Bundes } 1999 \text { für Forschung und Forschungsförderung nach sozio-ökonomischen } \\
\text { Zielsetzungen (ÖSTAT-Systematik) und Ressorts }\end{array}$ \\
\hline 7 & $\begin{array}{l}\text { Ausgaben des Bundes } 2000 \text { für Forschung und Forschungsförderung nach sozio-ökonomischen } \\
\text { Zielsetzungen (ÖSTAT-Systematik) und Ressorts }\end{array}$ \\
\hline 8 & $\begin{array}{l}\text { Ausgaben des Bundes } 2001 \text { für Forschung und Forschungsförderung nach sozio-ökonomischen } \\
\text { Zielsetzungen (ÖSTAT-Systematik) und Ressorts; ohne die im BFG } 2001 \text { veranschlagten Mittel der } \\
\text { "Forschungs- und Technologieoffensive" }\end{array}$ \\
\hline 9 & $\begin{array}{l}\text { Forschungsförderungen und Forschungsaufträge des Bundes } 1999 \text { nach Förderungsempfängern } \\
\text { bzw. Auftragnehmern (gegliedert nach volkswirtschaftlichen Sektoren/Bereichen) und vergebenden } \\
\text { Ressorts (einschließlich "große" Globalförderungen) }\end{array}$ \\
\hline 10 & $\begin{array}{l}\text { Forschungsförderungen und Forschungsaufträge des Bundes } 1999 \text { nach Förderungsempfängern } \\
\text { bzw. Auftragnehmern (gegliedert nach volkswirtschaftlichen Sektoren/Bereichen) und vergebenden } \\
\text { Ressorts (ohne "große" Globalförderungen) }\end{array}$ \\
\hline 11 & $\begin{array}{l}\text { Forschungsförderungen und Forschungsaufträge des Bundes } 1999 \text { nach sozio-ökonomischen Ziel- } \\
\text { setzungen und vergebenden Ressorts (einschließlich "große" Globalförderungen) }\end{array}$ \\
\hline 12 & $\begin{array}{l}\text { Forschungsförderungen und Forschungsaufträge des Bundes } 1999 \text { nach sozio-ökonomischen Ziel- } \\
\text { setzungen und vergebenden Ressorts (ohne "große" Globalförderungen) }\end{array}$ \\
\hline 13 & $\begin{array}{l}\text { Forschungsförderungen und Forschungsaufträge des Bundes } 1999 \text { nach Wissenschaftszweigen } \\
\text { und vergebenden Ressorts (einschließlich "große" Globalförderungen) }\end{array}$ \\
\hline 14 & $\begin{array}{l}\text { Forschungsförderungen und Forschungsaufträge des Bundes } 1999 \text { nach Wissenschaftszweigen } \\
\text { und vergebenden Ressorts (ohne "große" Globalförderungen) }\end{array}$ \\
\hline 15 & $\begin{array}{l}\text { Universitäts-Jahresrechnung 2001: Bundespersonal an Instituten und Kliniken gegliedert nach Wis- } \\
\text { senschaftszweigen und Beschäftigtenkategorien (VZÄ-Schätzung auf der Basis des Stellenplanes } 1 . \\
\text { 1. 2001) }\end{array}$ \\
\hline 16 & $\begin{array}{l}\text { Universitäten: Beschäftigte in Forschung und experimenteller Entwicklung (in Vollzeitäquivalent) } \\
\text { an Instituten und Kliniken im Jahre } 1998 \text { nach Wissenschaftszweigen und Beschäftigtenkategorien }\end{array}$ \\
\hline 17 & $\begin{array}{l}\text { Universitäten: Arbeitszeitverteilung der an Instituten und Kliniken im Jahre } 1998 \text { Beschäftigten nach } \\
\text { Wissenschaftszweigen und Beschäftigtenkategorien }\end{array}$ \\
\hline 18 & $\begin{array}{l}\text { Universitäten: Wissenschaftliches Personal (in VZÄ für F\&E) an Instituten und Kliniken im Jahre } \\
1998 \text { nach Wissenschaftszweigen, Beschäftigtenkategorien, Alter und Geschlecht }\end{array}$ \\
\hline 19 & $\begin{array}{l}\text { Beschäftigte in Forschung und experimenteller Entwicklung in sämtlichen Erhebungsbereichen im } \\
\text { Jahre } 1998 \text { in Kopfzahlen und Vollzeitäquivalenten nach Beschäftigtenkategorien }\end{array}$ \\
\hline 20 & $\begin{array}{l}\text { Beschäftigte in Forschung und experimenteller Entwicklung in sämtlichen Erhebungsbereichen im } \\
\text { Jahre } 1998 \text { in Kopfzahlen und Vollzeitäquivalenten nach Beschäftigtenkategorien und Geschlecht }\end{array}$ \\
\hline 21 & $\begin{array}{l}\text { Hochschulsektor: Beschäftigte in Forschung und experimenteller Entwicklung im Jahre } 19 \\
\text { Wissenschaftszweigen und Beschäftigtenkategorien }\end{array}$ \\
\hline
\end{tabular}




\begin{tabular}{|c|c|}
\hline 22 & $\begin{array}{l}\text { Sektor Staat: Beschäftigte in Forschung und experimenteller Entwicklung im Jahre } 1998 \text { nach Wis- } \\
\text { senschaftszweigen und Beschäftigtenkategorien }\end{array}$ \\
\hline 23 & $\begin{array}{l}\text { Sektor Staat: Beschäftigte in Forschung und experimenteller Entwicklung im Jahre } 1998 \text { nach } \\
\text { Rechtsträgern und Beschäftigtenkategorien }\end{array}$ \\
\hline 24 & $\begin{array}{l}\text { Privater gemeinnütziger Sektor: Beschäftigte in Forschung und experimenteller Entwicklung im } \\
\text { Jahre } 1998 \text { nach Wissenschaftszweigen und Beschäftigtenkategorien }\end{array}$ \\
\hline 25 & $\begin{array}{l}\text { Unternehmenssektor ohne firmeneigener Bereich: Beschäftigte in Forschung und experimenteller } \\
\text { Entwicklung im Jahre } 1998 \text { nach Wissenschaftszweigen und Beschäftigtenkategorien }\end{array}$ \\
\hline 26 & $\begin{array}{l}\text { Forschung und experimentelle Entwicklung nach Wissenschaftszweigen: Zusammenfassung der } \\
\text { Erhebungsbereiche unter Ausschluss des firmeneigenen Bereichs. Beschäftigte in F\&E in den Jah- } \\
\text { ren 1989, } 1993 \text { und } 1998 \text {. }\end{array}$ \\
\hline 27 & $\begin{array}{l}\text { Forschung und experimentelle Entwicklung im firmeneigenen Bereich 1998: Beschäftigte in F\&E } \\
\text { und Ausgaben für F\&E } 1998 \text { nach Wirtschaftszweigen (ÖNACE) }\end{array}$ \\
\hline 28 & Forschung und experimentelle Entwicklung (F\&E) 1998 im internationalen Vergleich \\
\hline 29 & FWF: Bewilligungen nach Forschungsstätten 2000 \\
\hline 30 & FWF: Bewilligungen nach Forschungsstätten - Zahl der Neubewilligungen 2000 \\
\hline 31 & FWF: Förderungskategorien - Neu- und Zusatzbewilligungen 2000 (in Mio. ATS) \\
\hline 32 & FWF: Bewilligungen 2000 nach Wissenschaftsdisziplinen \\
\hline 33 & FFF: Förderungsübersicht 2000 nach der Systematik der Wirtschaftstätigkeit (NACE) \\
\hline 34 & FFF: Förderungsübersicht 2000 nach Sonderbereichen der Forschung \\
\hline 35 & FFF: Förderungsübersicht 2000 nach Bundesländern (Projektstandort) \\
\hline
\end{tabular}


Tabelle 1: Globalschätzung 2001: Bruttoinlandsausgaben für F\&E

Finanzierung der in Österreich durchgeführten Forschung und experimentellen Entwicklung 1981 - 2001 (in Mio. Schilling)

\begin{tabular}{|c|c|c|c|c|c|c|c|c|c|c|c|c|c|}
\hline & Finanzierungssektoren & 1981 & 1985 & 1989 & 1993 & 1994 & 1995 & 1996 & 1997 & 1998 & 1999 & 2000 & 2001 \\
\hline \multirow[t]{6}{*}{1.} & $\begin{array}{l}\text { Bruttoinlandsausgaben für F\&E } \\
\text { (in Mio. ATS) } \\
\quad \text { Davon finanziert durch: }\end{array}$ & 12332,1 & 17183,2 & 22966,9 & 31694,2 & 34943,5 & 36876,9 & 39291,0 & 42450,2 & 47258,1 & 49533,9 & 50740,6 & 53967,6 \\
\hline & A. Bund ${ }^{1}$ ) & 4986,7 & 7130,2 & 8501,7 & 13170,2 & 14794,2 & 15030,1 & 14674,8 & 14828,0 & 15560,4 & 16523,7 & 16409,6 & 18438,9 \\
\hline & B. Bundesländer ${ }^{2}$ ) & 658,6 & 979,7 & 1229,9 & 1784,3 & 2183,6 & 2117,5 & 2188,7 & 2302,8 & 2582,6 & 2744,1 & 2934,8 & 3064,4 \\
\hline & C. Unternehmenssektor ${ }^{3}$ ) & 6195,9 & 8440,8 & 12182,6 & 15527,1 & 16073,6 & 16688,1 & 17366,8 & 18107,0 & 19125,8 & 19871,0 & 20804,1 & 21627,2 \\
\hline & D. Ausland ${ }^{4}$ ) & 305,0 & 425,2 & 741,3 & 821,4 & 1466,0 & 2602,0 & 4616,7 & 6559,2 & 9447,4 & 9842,8 & 10058,8 & 10401,9 \\
\hline & E. Sonstige ${ }^{5}$ ) & 185,9 & 207,3 & 311,4 & 391,2 & 426,1 & 439,2 & 444,0 & 653,2 & 541,9 & 552,3 & 533,3 & 435,2 \\
\hline 2. & $\begin{array}{l}\text { BIP nominell }{ }^{6} \text { ) } \\
\text { (in Mrd. ATS) }\end{array}$ & 1081,7 & 1369,1 & 1699,1 & 2159,5 & 2276,1 & 2370,7 & 2450,0 & 2513,5 & 2614,7 & 2712,0 & 2833,9 & 2941,4 \\
\hline 3. & $\begin{array}{l}\text { Bruttoinlandsausgaben für F\&E } \\
\text { in } \% \text { des BIP }\end{array}$ & 1,14 & 1,26 & 1,35 & 1,47 & 1,54 & 1,56 & 1,60 & 1,69 & 1,81 & 1,83 & 1,79 & 1,83 \\
\hline
\end{tabular}

Stand: April 2001

Quelle: Statistik Austria (Bundesanstalt Statistik Österreich)

1) 1981, 1985, 1989 und 1993: Erhebungsergebnisse (Bund einschließlich Mittel der zwei Forschungsförderungsfonds sowie 1989 und 1993 auch einschließlich des ITF).

1990 - 1992 und 1994 - 2001: Beilage T/Teil b (Bundesbudget-Forschung); 1990 zusätzlich Sonderaktion zur Förderung außenhandelsorientierter F\&E-Vorhaben (50,0 Mio.ATS).

2000: Einschließlich jener Rücklagenreste aus den Technologiemilliarden 1997 und 1998, die im Jahr 2000 für Forschungszwecke in Anspruch genommen wurden (Nicht im Bundesvoranschlag 2000 enthalten).

2001: Im Bundesbudget Forschung sind für 2001 unter dem VA-Ansatz 1/5182 12 für das F\&E-Offensivprogramm 7 Mrd. ATS veranschlagt. Aufgrund der bisher vorliegenden Informationen wird von der Annahme ausgegangen, daß von diesen 7 Mrd. ATS im Jahre 2001 nur ca. 2 Mrd. ATS ausgegeben werden, die Inanspruchnahme der restlichen 5 Mrd. ATS ist für F\&E-Ausgaben der Folgejahre vorgesehen. In die F\&E-Ausgaben-Schätzung von Statistik Austria sind daher - abweichend von der Beilage T/Teil b - nur jene 2 Mrd. ATS, welche im Jahre 2001 der Forschung zufließen sollen, eingegangen.

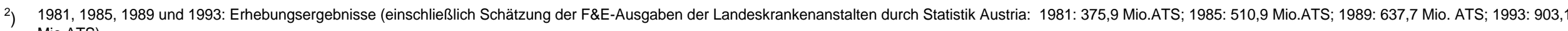
Mio.ATS).

1990 - 1992 und 1994 - 2001: Schätzung durch Statistik Austria unter Heranziehung der F\&E-Ausgaben-Schätzungen der Ämter der Landesregierungen.

3) Umfaßt Finanzierung durch die Wirtschaft (einschl. Jubiläumsfonds der Oesterreichischen Nationalbank).

1981, 1985, 1989 und 1993: Erhebungsergebnisse.

1990 - 1992 und 1994 - 2001: Schätzung durch Statistik Austria auf der Basis der Ergebnisse der F\&E-Erhebungen der Wirtschaftskammer Österreich (1989, 1991 und 1993$)$ und von Statistik Austria (1989, 1993 und 1998 Ergebnisse der F\&E-Erhebung von Statistik Austria im firmeneigenen Bereich).

4) 1981, 1985, 1989 und 1993: Erhebungsergebnisse. 1990 - 1992 und 1994 - 2001: Schätzung durch Statistik Austria unter Berücksichtigung der Ergebnisse der F\&E-Erhebung 1998 von Statistik Austria im firmeneigenen Bereich.

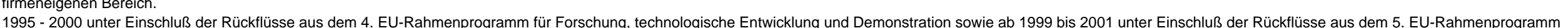
für Forschung, technologische Entwicklung und Demonstration (Stand: April 2001).

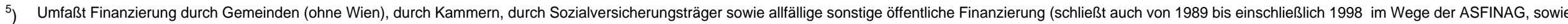
1993 bis einschließlich 2000 durch die BIG außerbudgetär finanzierte Bauvorhaben im Hochschulsektor mit ein) und durch den privaten gemeinnützigen Sektor.

1981, 1985, 1989 und 1993: Erhebungsergebnisse. 1990 - 1992 und 1994 - 2001: Schätzung durch Statistik Austria.

6) 1981 - 1999: Statistik Austria; 2000, 2001: WIFO-Prognose (März 2001) 
Tabelle 1a: Globalschätzung 2001: Bruttoinlandsausgaben für F\&E

Finanzierung der in Österreich durchgeführten Forschung und experimentellen Entwicklung 1981 - 2001 (in Prozent des BIP)

\begin{tabular}{|c|c|c|c|c|c|c|c|c|c|c|c|c|}
\hline Finanzierungssektoren & 1981 & 1985 & 1989 & 1993 & 1994 & 1995 & 1996 & 1997 & 1998 & 1999 & 2000 & 2001 \\
\hline $\begin{array}{l}\text { 1. Bruttoinlandsausgaben für F\&E } \\
\text { (in \% des BIP) } \\
\text { Davon finanziert durch: }\end{array}$ & 1,14 & 1,26 & 1,35 & 1,47 & 1,54 & 1,56 & 1,60 & 1,69 & 1,81 & 1,83 & 1,79 & 1,83 \\
\hline A. Bund ${ }^{1}$ ) & 0,46 & 0,52 & 0,50 & 0,61 & 0,65 & 0,63 & 0,60 & 0,59 & 0,60 & 0,61 & 0,58 & 0,63 \\
\hline B. Bundesländer ${ }^{2}$ ) & 0,06 & 0,07 & 0,07 & 0,08 & 0,10 & 0,09 & 0,09 & 0,09 & 0,10 & 0,10 & 0,10 & 0,10 \\
\hline C. Unternehmenssektor ${ }^{3}$ ) & 0,57 & 0,62 & 0,72 & 0,72 & 0,71 & 0,70 & 0,71 & 0,72 & 0,73 & 0,73 & 0,73 & 0,74 \\
\hline D. Ausland ${ }^{4}$ ) & 0,03 & 0,03 & 0,04 & 0,04 & 0,06 & 0,11 & 0,19 & 0,26 & 0,36 & 0,36 & 0,35 & 0,35 \\
\hline E. Sonstige ${ }^{5}$ ) & 0,02 & 0,02 & 0,02 & 0,02 & 0,02 & 0,02 & 0,02 & 0,03 & 0,02 & 0,02 & 0,02 & 0,01 \\
\hline $\begin{array}{l}\text { 2. BIP nominell }{ }^{6} \text { ) } \\
\text { (in Mrd. ATS) }\end{array}$ & 1081,7 & 1369,1 & 1699,1 & 2159,5 & 2276,1 & 2370,7 & 2450,0 & 2513,5 & 2614,7 & 2712,0 & 2833,9 & 2941,4 \\
\hline
\end{tabular}

Stand: April 2001

Quelle: Statistik Austria (Bundesanstalt Statistik Österreich)

1) 1981, 1985, 1989 und 1993: Erhebungsergebnisse (Bund einschließlich Mittel der zwei Forschungsförderungsfonds sowie 1989 und 1993 auch einschließlich des ITF).

1990 - 1992 und 1994 - 2001: Beilage T/Teil b (Bundesbudget-Forschung); 1990 zusätzlich Sonderaktion zur Förderung außenhandelsorientierter F\&E-Vorhaben (50,0 Mio.ATS).

2000: Einschließlich jener Rücklagenreste aus den Technologiemilliarden 1997 und 1998, die im Jahr 2000 für Forschungszwecke in Anspruch genommen wurden (Nicht im Bundesvoranschlag 2000 enthalten).

2001: Im Bundesbudget Forschung sind für 2001 unter dem VA-Ansatz 1/5182 12 für das F\&E-Offensivprogramm 7 Mrd. ATS veranschlagt. Aufgrund der bisher vorliegenden Informationen wird von der Annahme ausgegangen, daß von diesen 7 Mrd. ATS im Jahre 2001 nur ca. 2 Mrd. ATS ausgegeben werden, die Inanspruchnahme der restlichen 5 Mrd. ATS ist für F\&E-Ausgaben der Folgejahre vorgesehen. In die F\&E-Ausgaben-Schätzung von Statistik Austria sind daher - abweichend von der Beilage T/Teil b - nur jene 2 Mrd. ATS, welche im Jahre 2001 der Forschung zufließen sollen, eingegangen.

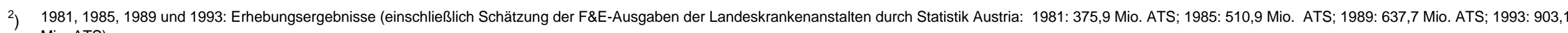

1990 - 1992 und 1994 - 2001: Schätzung durch Statistik Austria unter Heranziehung der F\&E-Ausgaben-Schätzungen der Ämter der Landesregierungen.

3) Umfaßt Finanzierung durch die Wirtschaft (einschl. Jubiläumsfonds der Oesterreichischen Nationalbank).

1990 - 1992 und 1994 - 2001: Schätzung durch Statistik Austria auf der Basis der Ergebnisse der F\&E-Erhebungen der Wirtschaftskammer Österreich (1989, 1991 und 1993) und von Statistik Austria (1989, 1993 und 1998 -

Ergebnisse der F\&E-Erhebung von Statistik Austria im firmeneigenen Bereich).

4) 1981, 1985, 1989 und 1993: Erhebungsergebnisse. 1990 - 1992 und 1994 - 2001: Schätzung durch Statistik Austria unter Berücksichtigung der Ergebnisse der F\&E-Erhebung 1998 von Statistik Austria im firmeneigenen Bereich.

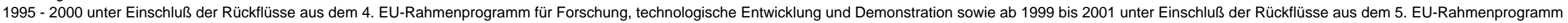
für Forschung, technologische Entwicklung und Demonstration (Stand: April 2001).

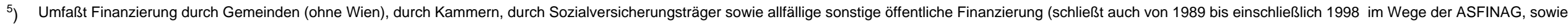
1993 bis einschließlich 2000 durch die BIG außerbudgetär finanzierte Bauvorhaben im Hochschulsektor mit ein) und durch den privaten gemeinnützigen Sektor.

1981, 1985, 1989 und 1993: Erhebungsergebnisse. 1990 - 1992 und 1994 - 2001: Schätzung durch Statistik Austria.

6) 1981 - 1999: Statistik Austria; 2000, 2001: WIFO-Prognose (März 2001). 
Tabelle 2: Ausgaben des Bundes für Forschung und Forschungsförderung 1998 bis 2001 nach Ressorts

Aufgliederung der Beilage T der Amtsbehelfe zu den Bundesfinanzgesetzen 2000 und 2001 (Teil a und Teil b)

\begin{tabular}{|c|c|c|c|c|c|c|c|c|}
\hline \multirow{3}{*}{ Ressorts ${ }^{1}$ ) } & \multicolumn{4}{|c|}{ Erfolg } & \multicolumn{4}{|c|}{ Bundesvoranschlag } \\
\hline & $\left.1998^{2}\right)$ & & $\left.1999^{3}\right)$ & & $\left.2000^{3}\right)$ & & $\left.2001^{3}\right)$ & \\
\hline & Mio. S & $\%$ & Mio. S & $\%$ & Mio. S & $\%$ & Mio. S & $\%$ \\
\hline Bundeskanzleramt & 109,382 & 0,7 & 108,364 & 0,6 & 77,907 & 0,5 & 70,521 & 0,3 \\
\hline Bundesministerium für Inneres & 2,358 & 0,0 & 2,235 & 0,0 & 1,994 & 0,0 & 1,994 & 0,0 \\
\hline Bundesministerium für Unterricht und kulturelle Angelegenheiten ${ }^{4}$ ) & 529,690 & 3,2 & 585,471 & 3,4 & . & . & · & . \\
\hline Bundesministerium für Wissenschaft und Verkehr ${ }^{4}$ ) & 11839,041 & 72,5 & 12474,876 & 72,0 & - &. & & \\
\hline Bundesministerium für Bildung, Wissenschaft und Kultur ${ }^{4}$ ) & & & & & 12546,775 & 76,3 & 12957,420 & 53,9 \\
\hline Bundesministerium für Arbeit, Gesundheit und Soziales & 1890,951 & 11,5 & 2041,780 & 11,8 & . & . & . & 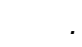 \\
\hline Bundesministerium für soziale Sicherheit und Generationen & & . & . & . & 483,920 & 2,9 & 145,958 & 0,6 \\
\hline Bundesministerium für Umwelt, Jugend und Familie & 171,476 & 1,0 & 170,966 & 1,0 & . & . & . & . \\
\hline Bundesministerium für auswärtige Angelegenheiten & 24,035 & 0,1 & 25,069 & 0,1 & 27,570 & 0,2 & 27,570 & 0,1 \\
\hline Bundesministerium für Justiz & 1,050 & 0,0 & 1,000 & 0,0 & 0,850 & 0,0 & 0,850 & 0,0 \\
\hline Bundesministerium für Landesverteidigung & 3,188 & 0,0 & 2,611 & 0,0 & 3,267 & 0,0 & 3,547 & 0,0 \\
\hline Bundesministerium für Finanzen & 398,825 & 2,4 & 415,705 & 2,4 & 429,933 & 2,6 & 7442,947 & 31,0 \\
\hline Bundesministerium für Land- und Forstwirtschaft ${ }^{4}$ ) & 531,007 & 3,2 & 581,074 & 3,3 & . & . & . & . \\
\hline Bundesministerium für Land- und Forstwirtschaft, Umwelt und Wasserwirtschaft $\left.{ }^{4}\right)^{5}$ ) & & . & . & . & 731,108 & 4,4 & 725,487 & 3,0 \\
\hline Bundesministerium für wirtschaftliche Angelegenheiten ${ }^{6}$ ) & 884,603 & 5,4 & 940,299 & 5,4 & . & . & . & . \\
\hline Bundesministerium für Wirtschaft und Arbeit ${ }^{6}$ ) & . & . & . & . & 89,063 & 0,5 & 93,855 & 0,4 \\
\hline Bundesministerium für Verkehr, Innovation und Technologie & . & . & . & . & 2075,895 & 12,6 & 2571,379 & 10,7 \\
\hline Bundesministerium für öffentliche Leistung und Sport & & . & . & . & - & - & - & - \\
\hline INSGESAMT & 16385,606 & 100,0 & 17349,450 & 100,0 & 16468,282 & 100,0 & 24041,528 & 100,0 \\
\hline
\end{tabular}

\section{Stand: April 2001}

Quelle: Statistik Austria (Bundesanstalt Statistik Österreich)

1) Entsprechend der im jeweiligen Jahr gültigen Fassung des Bundesministeriengesetzes 1986 (1998, 1999: BGBI. I Nr. 21/1997; 2000, 2001: BGBI. I Nr. 16/2000).

2) Amtsbehelf zum Bundesfinanzgesetz 2000.

3) Amtsbehelf zum Bundesfinanzgesetz 2001.

${ }^{4}$ ) Einschließlich der VA-Ansätze aus Kapitel 64 für Bauausgaben für dem Bundesministerium unterstehende F\&E ausführende Bundeseinrichtungen.

5) Bundesvoranschlag 2000: Einschließlich der im Budgetkapitel 18 veranschlagten Ausgaben für Forschung und Forschungsförderung im Bereich Umwelt.

$\left.{ }^{6}\right)$ Onne die VA-Ansätze aus Kapitel 64 für Bauausgaben für einem anderen Bundesministerium unterstehende F\&E ausführende Bundeseinrichtungen. 


\section{BEILAGE T \\ des Amtsbehelfes zum Bundesfinanzgesetz 2001}

\section{Forschungswirksame Ausgaben des Bundes von 1999 bis 2001 nach Ressorts}

Die nachfolgenden Übersichten für die Jahre 1999 bis 2001 sind aufgegliedert nach

1. Beitragszahlungen aus Bundesmitteln an internationale Organisationen, die Forschung und Forschungsförderung (mit) als Ziel haben (Teil a)

2. sonstigen Ausgaben des Bundes für Forschung und Forschungsförderung (Teil b, Bundesbudget Forschung)

Für die Aufstellung dieser Ausgaben ist in erster Linie der Gesichtspunkt der Forschungswirksamkeit maßgebend, der inhaltlich über den Aufgabenbereich 12 „Forschung und Wissenschaft" hinausgeht und auf dem Forschungsbegriff des FrascatiHandbuches der OECD beruht, wie er im Rahmen der forschungsstatistischen Erhebungen von STATISTIK AUSTRIA zur Anwendung gelangt.

Forschungswirksame Anteile bei den Bundesausgaben finden sich daher nicht nur bei den Ausgaben des Aufgabenbereiches 12 "Forschung und Wissenschaft", sondern auch in zahlreichen anderen Aufgabenbereichen (z. B. 11/Erziehung und Unterricht, 13/Kunst, 34/Land- und Forstwirtschaft, 36/Industrie und Gewerbe, 43/Übrige Hoheitsverwaltung), bei denen die Zielsetzungen des betreffenden Aufgabenbereiches im Vordergrund stehen.

Zur Beachtung: Die Anmerkungen zu den nachfolgenden Übersichten finden sich im Anhang zur Beilage $T$. 
a) Beitragszahlungen aus Bundesmitteln an internationale Organisationen, die Forsehung und Forschungsförderung (mit) als Ziel haben

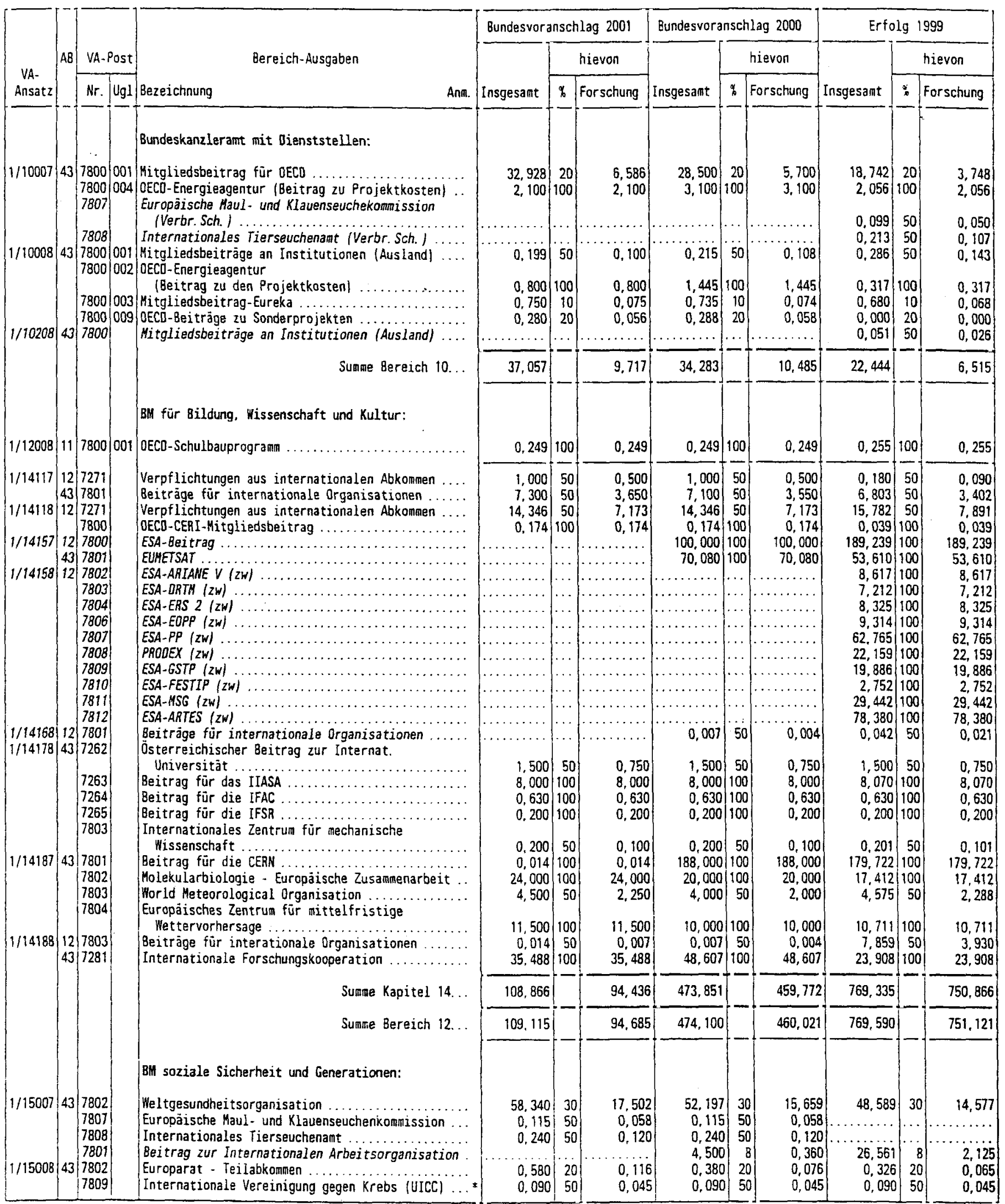


a) Beitragszahlungen aus Bundesmitteln an internationale Organisationen, die forschung und forschungsförderung (mit) als Ziel haben

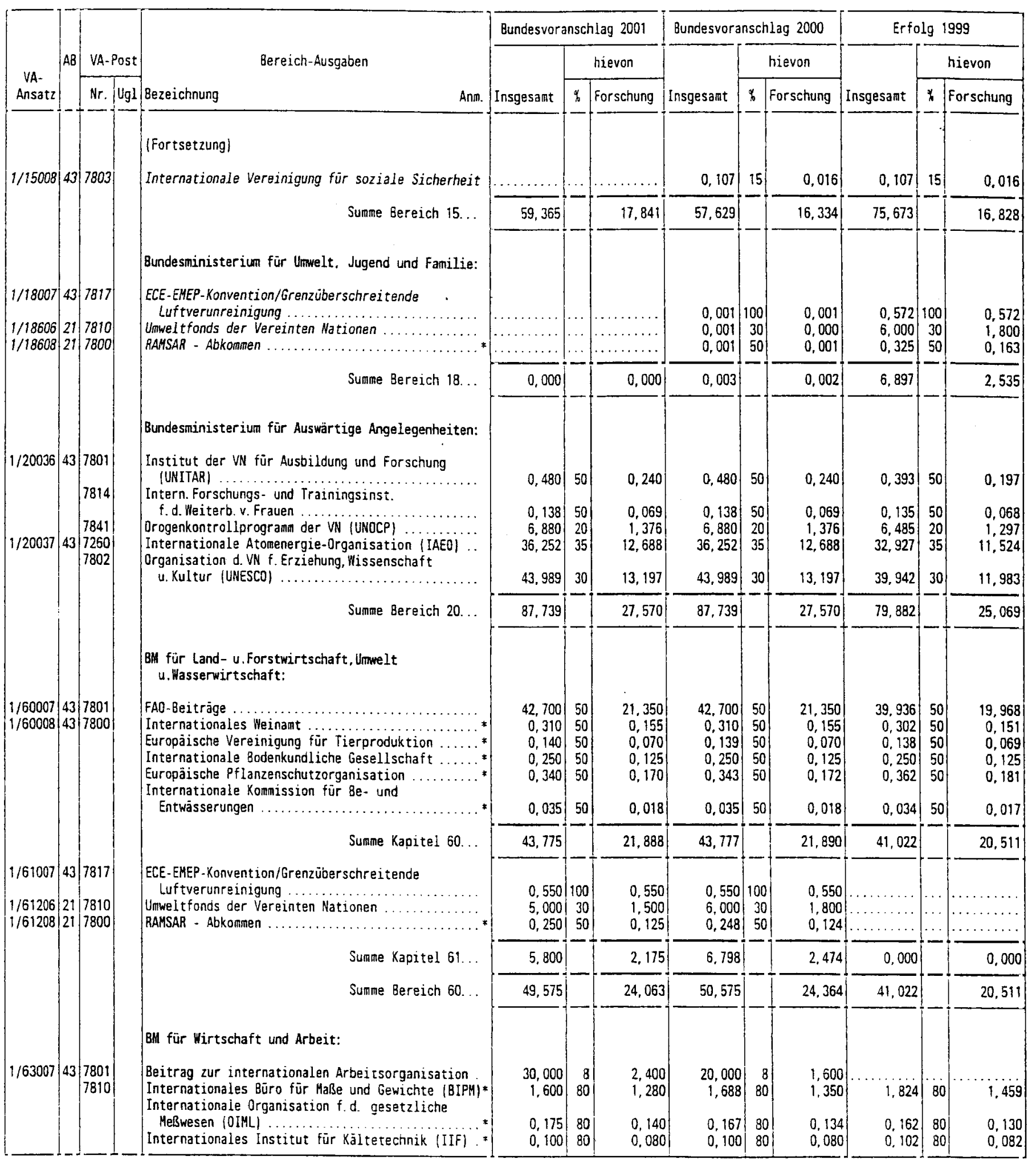


BUNOESVORANSCHLAG 2001

Forschungswirksame Ausgaben des Bundes $(*)$

(Beträge in Millionen Schilling)

a) Beitragszahlungen aus Bundesmitteln an internationale Organisationen, die forschung und Forschungsförderung (mit) als Ziel haben

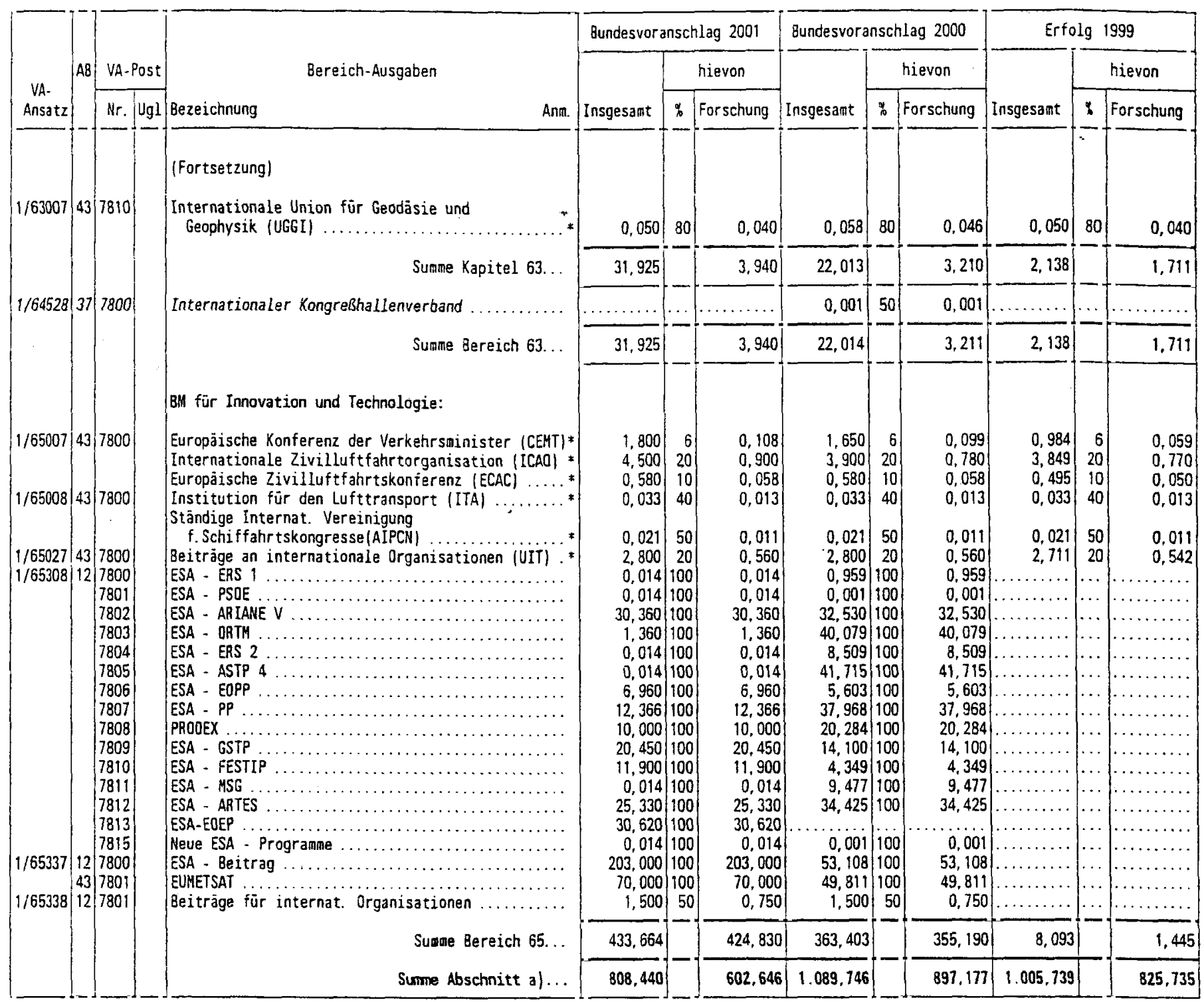


b) Ausgaben des Bundes (ausgen. die bereits im Abschnitt a) ausgewiesen sind) für Forschung und Forschungsförderung (Bundesbudget-Forschung)

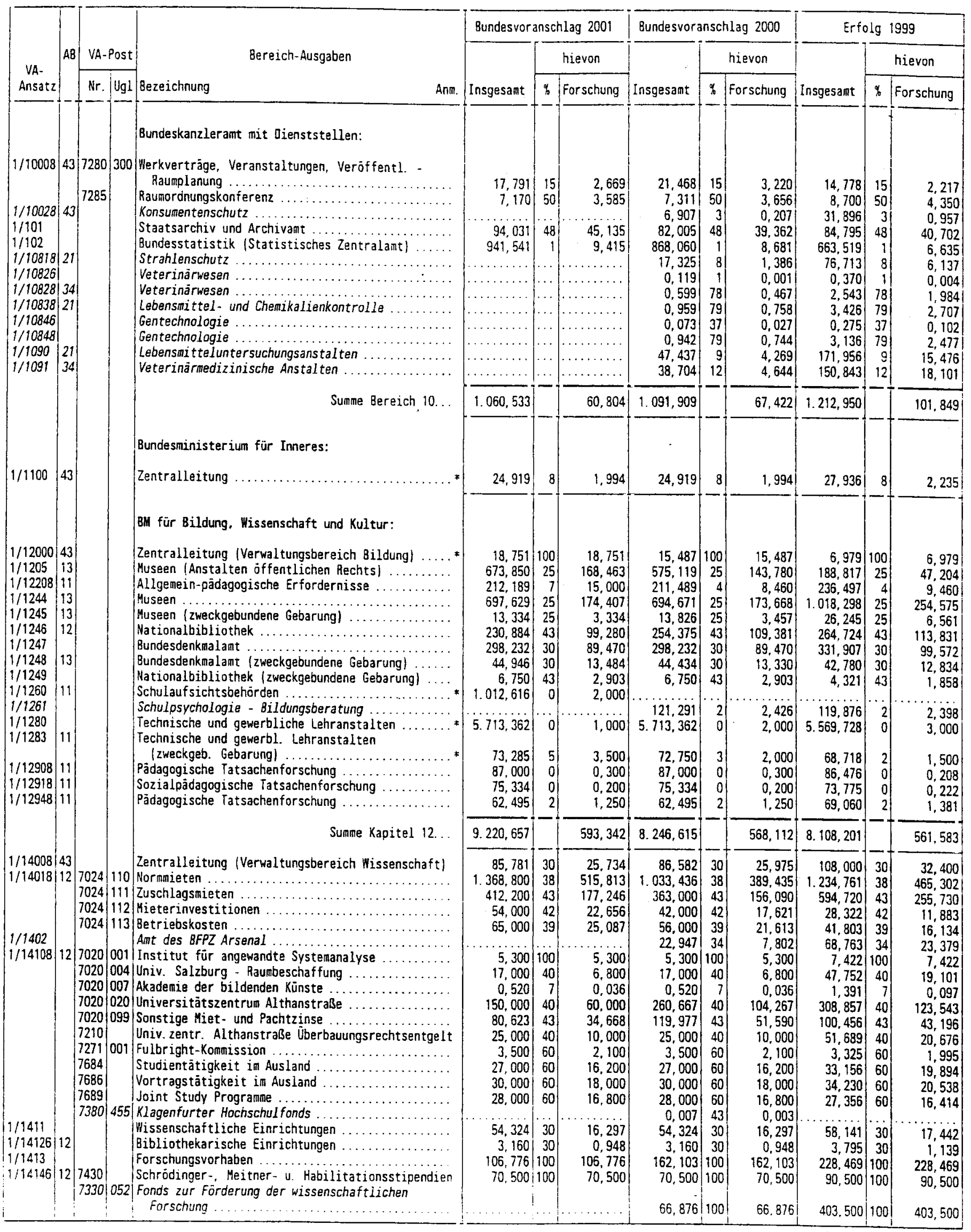


b) Ausgaben des Bundes (ausgen. die bereits im Abschnitt a) ausgewiesen sind) für Forschung und Forschungsförderung (Bundesbudget-Forschung/

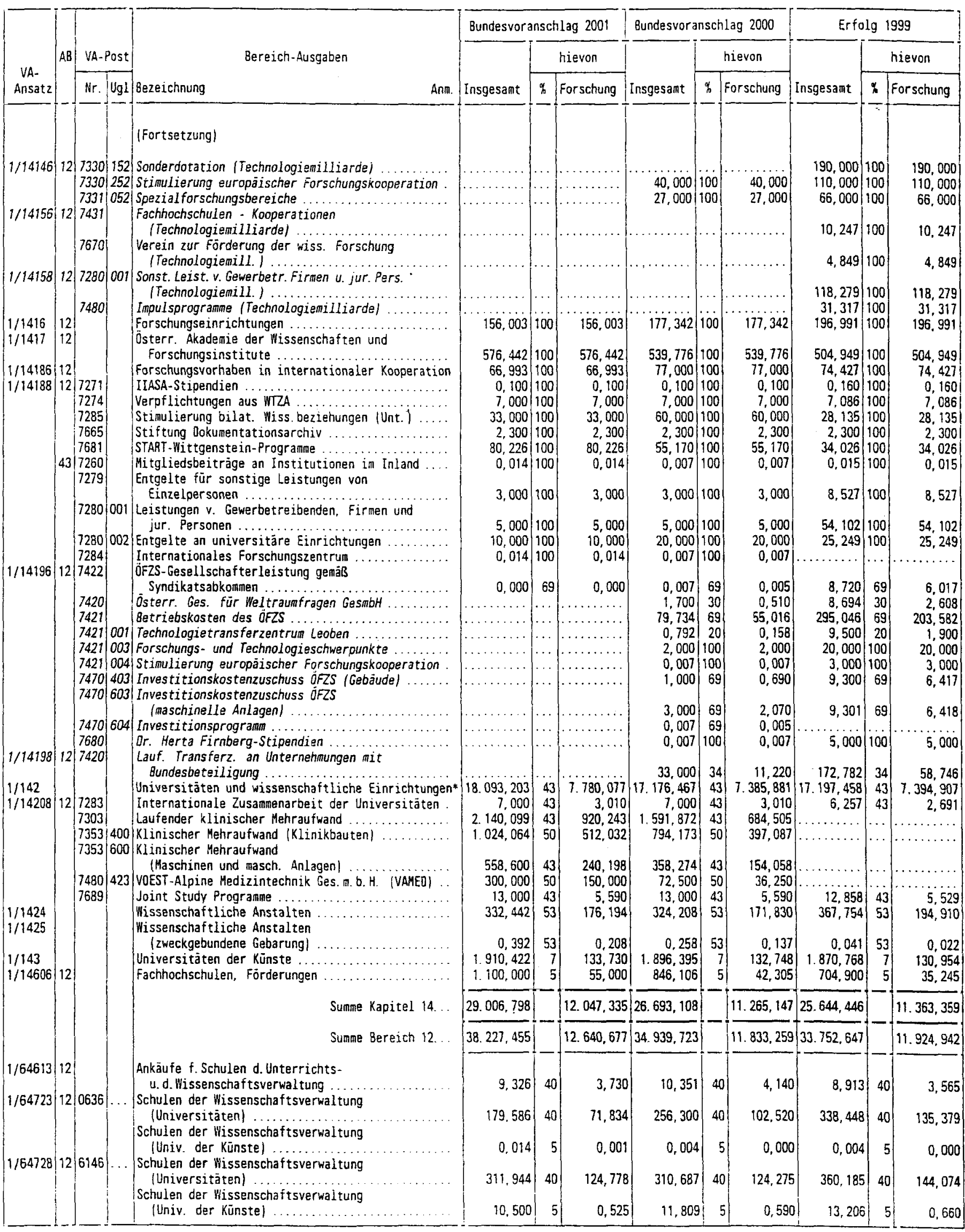


b) Ausgaben des Bundes (ausgen. die bereits im Abschnitt a) ausgewiesen sind) für Forschung und Forschungsförderung (Bundesbudget-Forschung)

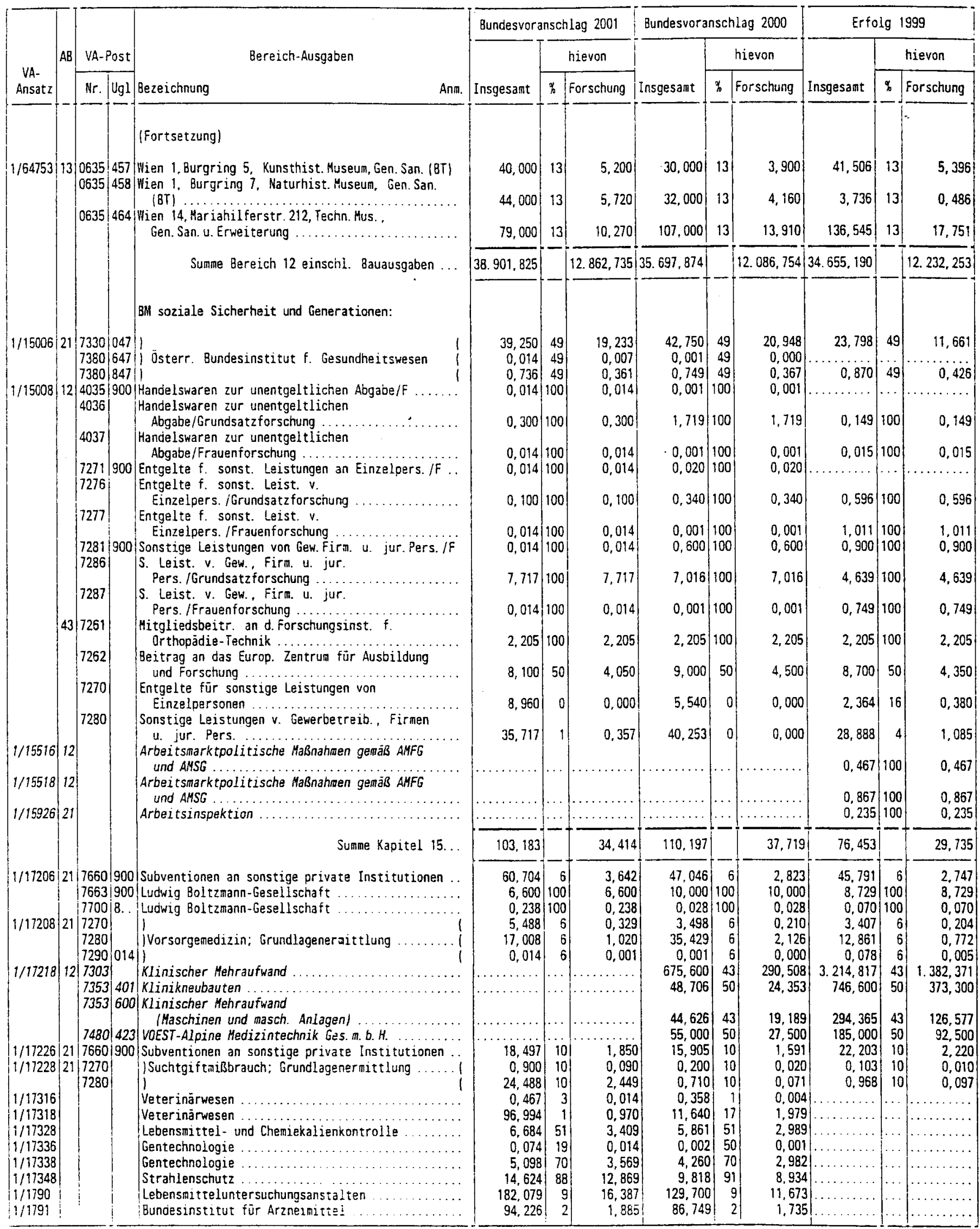


Beilage $T$

B UNOESVOAANSEHLAG 2001

Forschungswirksame Ausgaben des Bundes (*)

(Beträge in Millionen Schilling)

b) Ausgaben des 8undes (ausgen. die bereits in Abschnitt a) ausgewiesen sind) für Forschung und Forschungsförderung (Bundesbudget-Forschung)

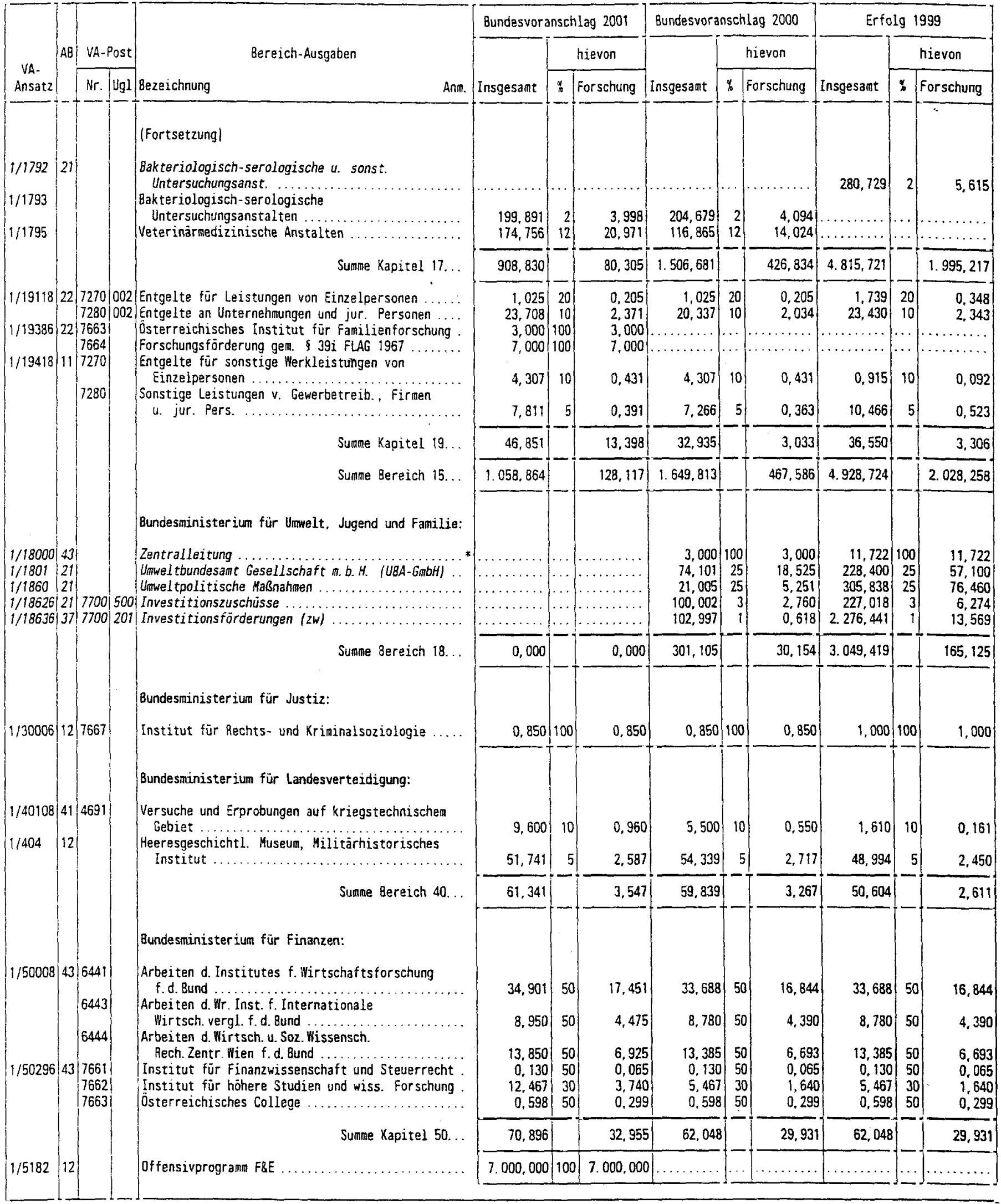


B UNOES VOA A S C H L A G 200

Forschungswirksame Ausgaben des Bundes (*) |Betràge in Millionen Schilling)

b) Ausgaben des Bundes (ausgen. die bereits im Abschnitt a) ausgewiesen sind) für Forschung und Forschungsförderung (Bundesbudget-Forschung)

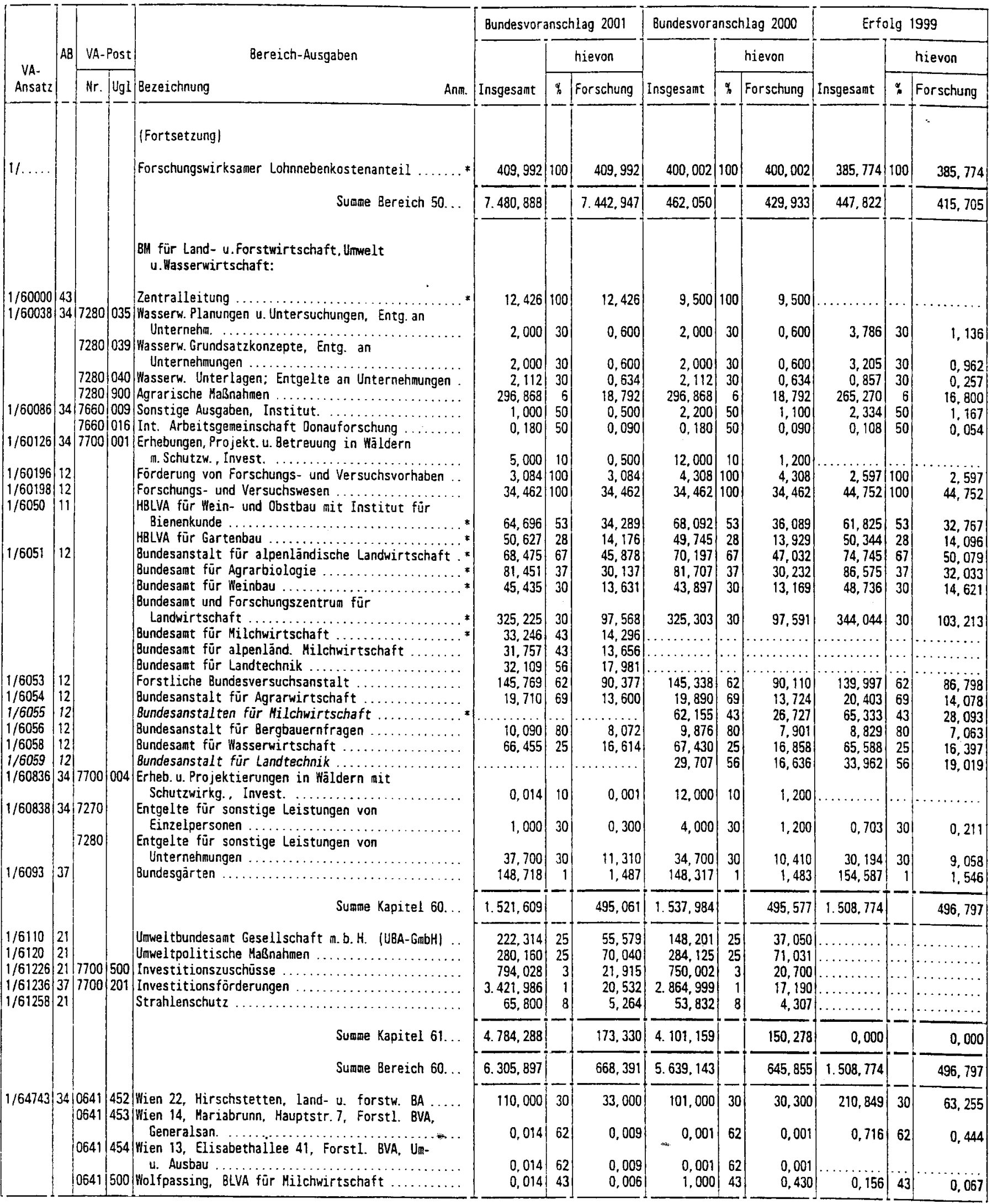


b) Ausgaben des Bundes (ausgen. die bereits in Abschnitt a) ausgewiesen sind) für Forschung und Forschungsförderung (Bundesbudget-Forschung)

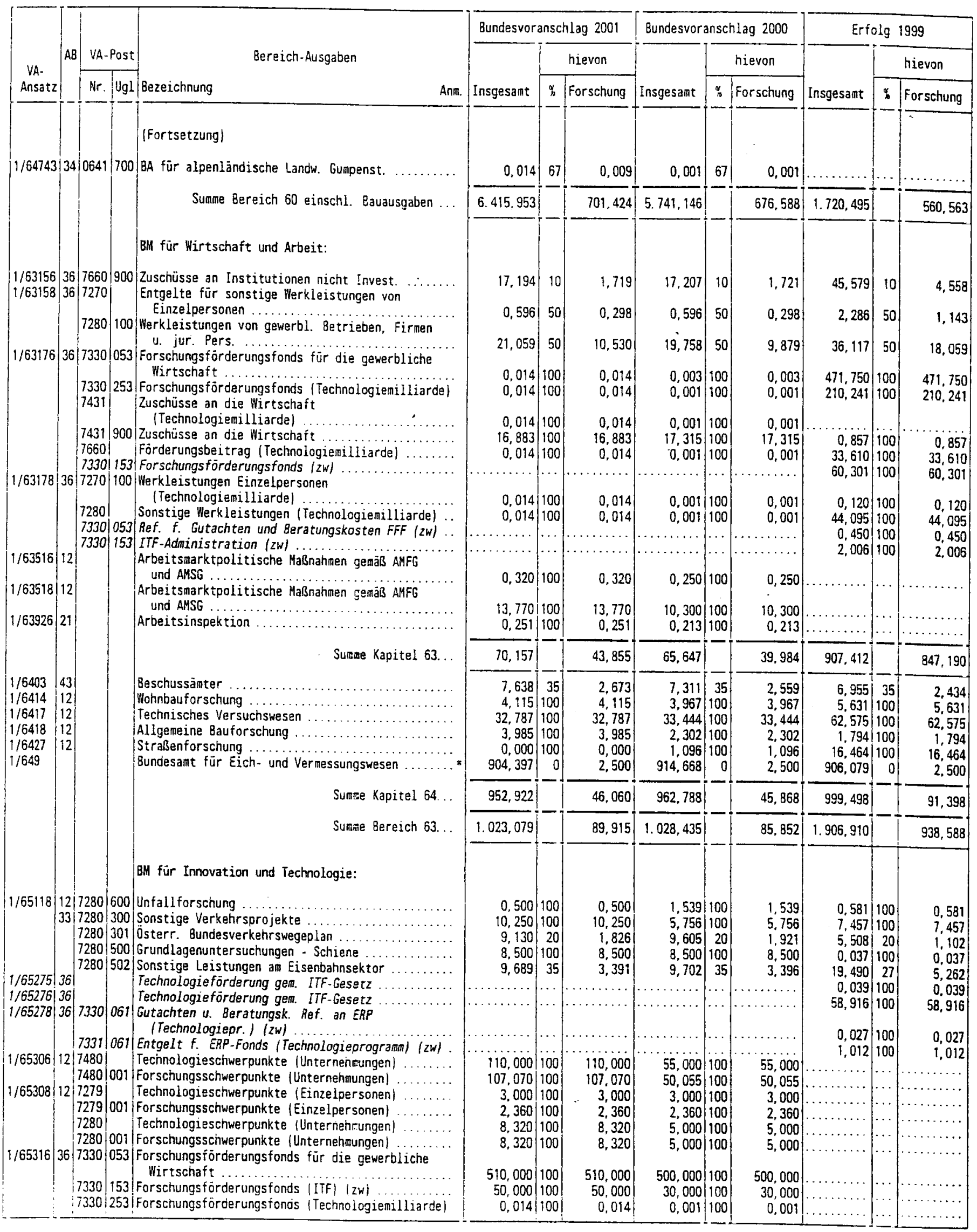


b) Ausgaben des Bundes (ausgen. die bereits in Abschnitt a) ausgewiesen sind) für Forschung und Forschungsförderung (Bundesbudget-Forschung)

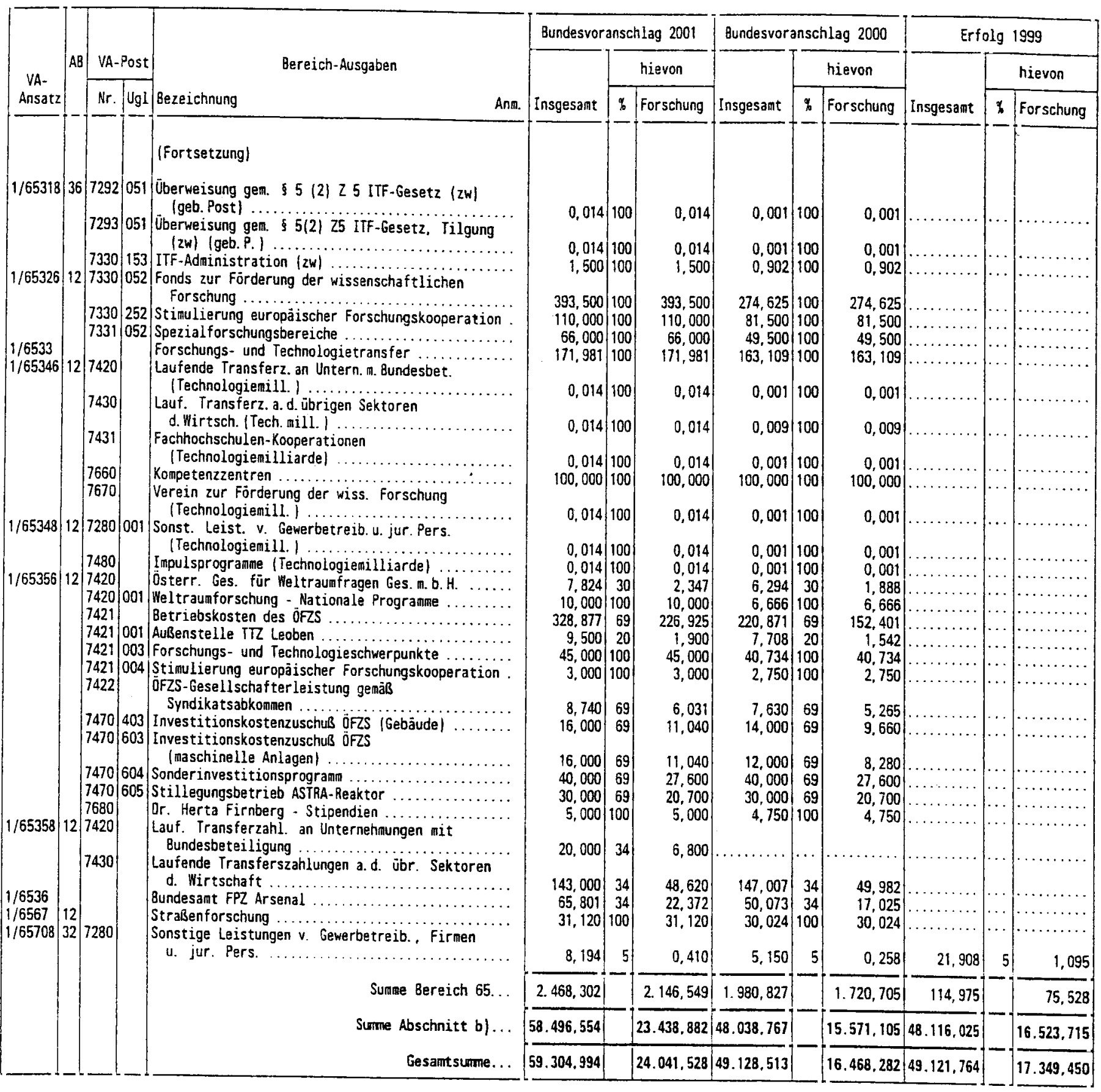


Armerkungen zur Beilage I

*) F \& E Koeffizienten geschätzt

Die Beilage I ist aufgegliedert nach:

a) Beitragszahlungen aus Bundesmitteln an internationale Organisationen, die forschung und Forschungsförderung (nit) als Ziel haben,

b) sonstigen Ausgaben des Bundes für Forschung und Forschungsförderung (Bundesbudget-Forschung)

Für die Aufstellung dieser Ausgaben ist in erster Linie der Gesichtspunkt der Forschungswirksankeit maßgebend, der inhaltlich über den Aufgabenbereich 12 'Forschung und Wissenschaft' hinausgeht und auf dem Forschungsbegriff des Frascati-Handbuches der OECD beruht, wie er im Rahmen der forschungsstatistischen Erhebungen des ÖSTAT zur Anwendung gelangt.

Forschungswirksame Anteile bei den Bundesausgaben finden sich daher nicht nur bei den Ausgaben des Aufgabenbereiches 12 'Forschung und Wissenschaft', sondern auch in zahlreichen anderen Aufgabenbereichen Zz. B. 11/Erziehung und Unterricht, 13/Kunst, 34/Land und Forstwirtschaft, 36/Industrie und Gewerbe, 43/Übrige Hoheitsverwaltungl, bei denen die Zielsetzungen des betreffenden Aufgabenbereiches in Vordergrund stehen.

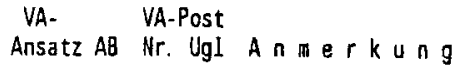

1/6055 12 Ohne Ausgaben der VA-Posten 7150, 7151 des VA-Ansatzes 1/60557 und der VA-Posten 4030, 4251 des VA-Ansatzes $1 / 60558$.

$1 / 61208217800 \quad$ Teilbetrag der VA-Post.

1/649 Forschungsanteil: Pauschalbetrag.

1/65007 437800 Teilbetrag der VA-Post.

$1 / 65008437800 \quad$ Teilbetrag der VA-Post.

$1 / 65027437800 \quad$ Teilbetrag der VA-Post.

$1 / \ldots$

F\&E-Anteil an den Lohnnebenkosten der in Forschungseinrichtungen tātigen Bundesbeanten. Imputation nach OECD-Richtlinien. 
Tabelle 4: Ausgaben des Bundes 1989 bis 2001 für Forschung und Forschungsförderung nach sozio-ökonomischen Zielsetzungen (ÖSTAT-Systematik) Aufgliederung der Beilage T des Amtsbehelfes zum jeweiligen Bundesfinanzgesetz (Teil a und Teil b)

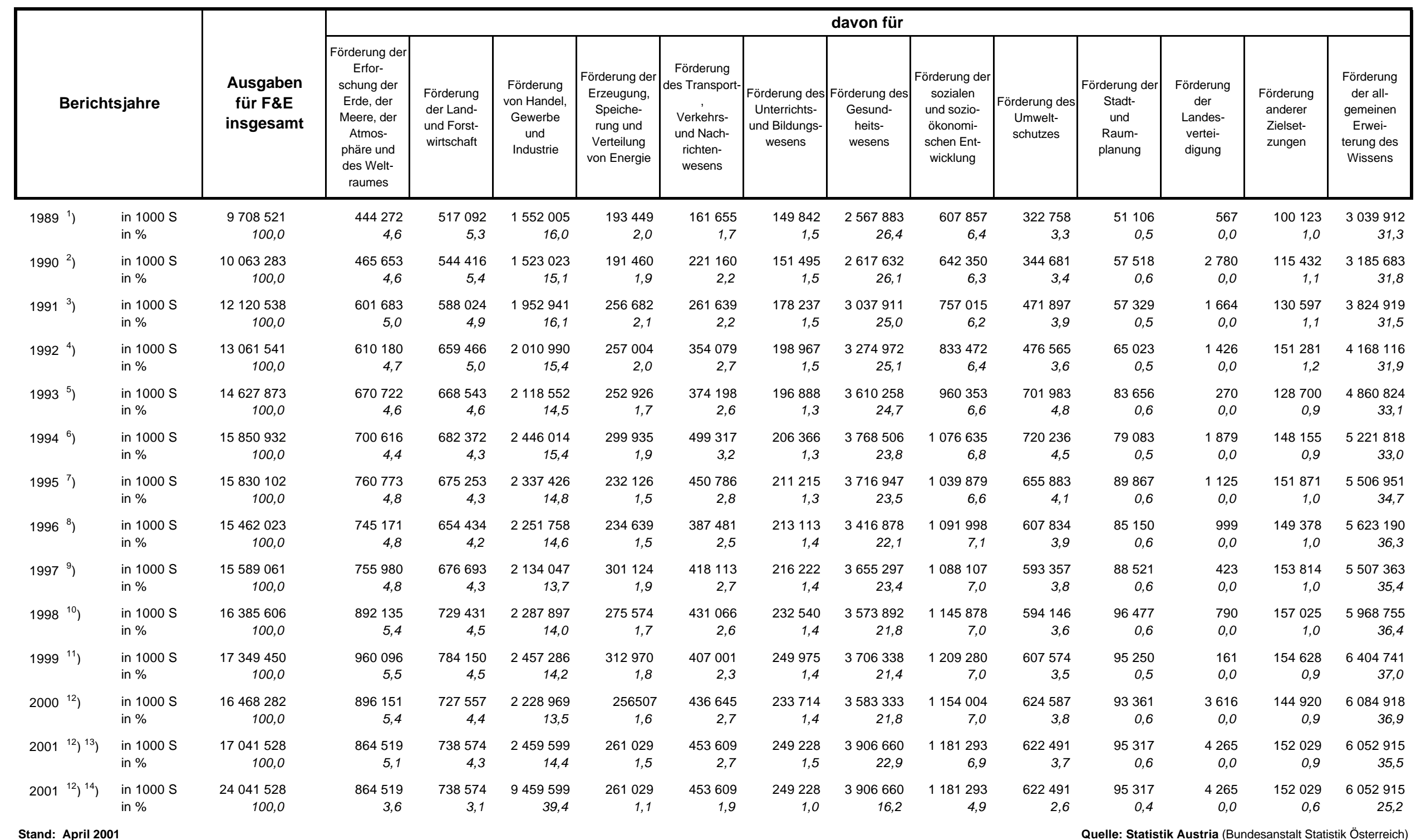

Stand: April 2001

1) Beilage T des Amtsbehelfes zum BFG 1991, Erfolg. Revidierte Daten. - ${ }^{2}$ ) Beilage T des Amtsbehelfes zum BFG 1992, Erfolg. Revidierte Daten. - ${ }^{3}$ ) Beilage T des Amtsbehelfes zum BFG 1993, Erfolg. Revidierte Daten

4) Beilage T des Amtsbehelfes zum BFG 1994, Erfolg. - ${ }^{5}$ ) Beilage T des Amtsbehelfes zum BFG 1995, Erfolg. Revidierte Daten. ${ }^{6}$ ) Beilage T des Amtsbehelfes zum BFG 1996, Erfolg. - ${ }^{7}$ ) Beilage T des Amtsbehelfes

zum BFG 1997, Erfolg. ${ }^{8)}$ Beilage T des Amtsbehelfes zum BFG 1998, Erfolg. - ${ }^{9}$ Beilage T des Amtsbehelfes zum BFG 1999, Erfolg. - ${ }^{10)}$ Beilage T des Amtsbehelfes zum BFG 2000, Erfolg. -

Beilage T des Amtsbehelfes zum BFG 2001, Erfolg. - ${ }^{22}$ Beilage T des Amtsbehelfes zum BFG 2001, Bundesvoranschlag. - ${ }^{-13}$ ) Ohne die im BFG 2001 rücklagefähig veranschlagten Mittel der Forschungs- und Technologieoffensive

(7 Mrd. Schilling). ${ }^{\left.-{ }^{4}\right)}$ Einschließlich der im BFG 2001 rücklagefähig veranschlagten Mittel der Forschungs- und Technologieoffensive (7 Mrd. Schilling). 
Tabelle 5: Ausgaben des Bundes 1998 für Forschung und Forschungsförderung nach sozio-ökonomischen Zielsetzungen (ÖSTAT-Systematik) und Ressorts Aufgliederung der Jahreswerte $1998^{1}$ ) aus der Beilage T des Amtsbehelfes zum Bundesfinanzgesetz 2000 (Teil a und Teil b)

\begin{tabular}{|c|c|c|c|c|c|c|c|c|c|c|c|c|c|c|c|}
\hline \multirow{2}{*}{\multicolumn{2}{|c|}{ RESSORTS }} & \multirow[b]{2}{*}{$\begin{array}{c}\text { Ausgaben } \\
\text { des Bundes } \\
\text { für F\&E } \\
\text { insgesamt }\end{array}$} & \multicolumn{13}{|c|}{ davon für } \\
\hline & & & $\begin{array}{c}\text { Förderung } \\
\text { der Erfor- } \\
\text { schung der } \\
\text { Erde, der } \\
\text { Meere, der } \\
\text { Atmos- } \\
\text { phäre und } \\
\text { des Welt- } \\
\text { raumes }\end{array}$ & $\begin{array}{l}\text { Förderung } \\
\text { der Land- } \\
\text { und Forst- } \\
\text { wirtschaft }\end{array}$ & $\begin{array}{c}\text { Förderung } \\
\text { von Handel, } \\
\text { Gewerbe } \\
\text { und } \\
\text { Industrie }\end{array}$ & \begin{tabular}{|c|} 
Förderung \\
der \\
Erzeugung, \\
Speiche- \\
rung und \\
Verteilung \\
von Energie
\end{tabular} & $\begin{array}{l}\text { Förderung } \\
\text { des } \\
\text { Transport-, } \\
\text { Verkehrs- } \\
\text { und Nach- } \\
\text { richten- } \\
\text { wesens }\end{array}$ & $\begin{array}{c}\text { Förderung } \\
\text { des } \\
\text { Unterrichts- } \\
\text { und Bildungs } \\
\text { wesens }\end{array}$ & $\begin{array}{l}\text { Förderung } \\
\text { des } \\
\text { Gesund- } \\
\text { heits- } \\
\text { wesens }\end{array}$ & $\begin{array}{l}\text { Förderung } \\
\text { der } \\
\text { sozialen } \\
\text { und sozio- } \\
\text { ökonomi- } \\
\text { schen Ent- } \\
\text { wicklung }\end{array}$ & $\begin{array}{c}\text { Förderung } \\
\text { des } \\
\text { Umwelt- } \\
\text { schutzes }\end{array}$ & $\begin{array}{c}\text { Förderung } \\
\text { der Stadt- } \\
\text { und } \\
\text { Raum- } \\
\text { planung }\end{array}$ & $\begin{array}{c}\text { Förderung } \\
\text { der } \\
\text { Landes- } \\
\text { vertei- } \\
\text { digung }\end{array}$ & $\begin{array}{c}\text { Förderung } \\
\text { anderer } \\
\text { Zielset- } \\
\text { zungen }\end{array}$ & $\begin{array}{l}\text { Förderung } \\
\text { der all- } \\
\text { gemeinen } \\
\text { Erwei- } \\
\text { terung des } \\
\text { Wissens }\end{array}$ \\
\hline BKA & $\begin{array}{l}\text { in } 1000 \mathrm{~S} \\
\text { in } \%\end{array}$ & $\begin{array}{r}109382 \\
100,0\end{array}$ & - & $\begin{array}{r}18016 \\
16,5\end{array}$ & $\begin{array}{r}64 \\
0,1\end{array}$ & $\begin{array}{r}2288 \\
2,1\end{array}$ & - & - & $\begin{array}{r}22167 \\
20,3\end{array}$ & $\begin{array}{r}13962 \\
12,8\end{array}$ & $\begin{array}{r}5911 \\
5,4\end{array}$ & $\begin{array}{r}7482 \\
6,8\end{array}$ & - & - & $\begin{array}{r}39492 \\
36,0\end{array}$ \\
\hline BMI & $\begin{array}{l}\text { in } 1000 \mathrm{~S} \\
\text { in } \%\end{array}$ & $\begin{array}{l}2358 \\
100,0\end{array}$ & - & - & $\begin{array}{l}- \\
-\end{array}$ & - & - & - & $\begin{array}{l}- \\
-\end{array}$ & $\begin{array}{l}2358 \\
100,0\end{array}$ & $\begin{array}{l}- \\
-\end{array}$ & - & - & - & - \\
\hline BMUKA & $\begin{array}{l}\text { in } 1000 \mathrm{~S} \\
\text { in } \%\end{array}$ & $\begin{array}{r}529690 \\
100,0\end{array}$ & $\begin{array}{l}- \\
-\end{array}$ & - & $\begin{array}{r}6000 \\
1,1\end{array}$ & $\begin{array}{l}- \\
-\end{array}$ & - & $\begin{array}{r}12245 \\
2,3\end{array}$ & - & $\begin{array}{r}108959 \\
20,6\end{array}$ & $\begin{array}{l}- \\
-\end{array}$ & - & - & $\begin{array}{l}- \\
-\end{array}$ & $\begin{array}{r}402486 \\
76,0\end{array}$ \\
\hline BMWV & $\begin{array}{l}\text { in } 1000 \mathrm{~S} \\
\text { in } \%\end{array}$ & $\begin{array}{r}11839041 \\
100,0\end{array}$ & $\begin{array}{r}865413 \\
7,3\end{array}$ & $\begin{array}{r}212737 \\
1,8\end{array}$ & $\begin{array}{r}1543979 \\
13,0\end{array}$ & $\begin{array}{r}199674 \\
1,7\end{array}$ & $\begin{array}{r}377760 \\
3,2\end{array}$ & $\begin{array}{r}213431 \\
1,8\end{array}$ & $\begin{array}{r}1505652 \\
12,7\end{array}$ & $\begin{array}{r}909138 \\
7,7\end{array}$ & $\begin{array}{r}389528 \\
3,3\end{array}$ & $\begin{array}{r}85722 \\
0,7\end{array}$ & - & $\begin{array}{r}151822 \\
1,3\end{array}$ & $\begin{array}{r}5384185 \\
45,5\end{array}$ \\
\hline BMAGS & $\begin{array}{l}\text { in } 1000 \mathrm{~S} \\
\text { in } \%\end{array}$ & $\begin{array}{r}1890951 \\
100,0\end{array}$ & - & - & - & - & - & - & $\begin{array}{r}1876575 \\
99,2\end{array}$ & $\begin{array}{r}14376 \\
0,8\end{array}$ & - & - & - & - & - \\
\hline BMUJF & $\begin{array}{l}\text { in } 1000 \mathrm{~S} \\
\text { in } \%\end{array}$ & $\begin{array}{r}171476 \\
100,0\end{array}$ & - & - & - & - & - & - & - & $\begin{array}{r}3076 \\
1,8\end{array}$ & $\begin{array}{r}168400 \\
98,2\end{array}$ & - & - & - & - \\
\hline BMAA & $\begin{array}{l}\text { in } 1000 \mathrm{~S} \\
\text { in } \%\end{array}$ & $\begin{array}{r}24035 \\
100,0\end{array}$ & - & - & - & $\begin{array}{r}10067 \\
41,9\end{array}$ & - & - & - & $\begin{array}{r}13783 \\
57,3\end{array}$ & - & - & - & - & $\begin{array}{c}185 \\
0,8\end{array}$ \\
\hline BMJ & $\begin{array}{l}\text { in } 1000 \mathrm{~S} \\
\text { in } \%\end{array}$ & $\begin{array}{l}1050 \\
100,0\end{array}$ & - & - & - & - & - & - & - & $\begin{array}{l}1050 \\
100,0\end{array}$ & - & - & $\begin{array}{l}- \\
-\end{array}$ & - & - \\
\hline BML & $\begin{array}{l}\text { in } 1000 \mathrm{~S} \\
\text { in } \%\end{array}$ & $\begin{array}{l}3188 \\
100,0\end{array}$ & - & - & - & - & - & - & - & - & - & - & $\begin{array}{r}790 \\
24,8\end{array}$ & - & $\begin{array}{r}2398 \\
75,2\end{array}$ \\
\hline BMF & $\begin{array}{l}\text { in } 1000 \mathrm{~S} \\
\text { in } \%\end{array}$ & $\begin{array}{r}398825 \\
100,0\end{array}$ & $\begin{array}{r}21788 \\
5,5\end{array}$ & $\begin{array}{r}22646 \\
5,7\end{array}$ & $\begin{array}{r}42971 \\
10,8\end{array}$ & $\begin{array}{r}6179 \\
1,5\end{array}$ & $\begin{array}{r}5197 \\
1,3\end{array}$ & $\begin{array}{r}6864 \\
1,7\end{array}$ & $\begin{array}{r}82475 \\
20,7\end{array}$ & $\begin{array}{r}57945 \\
14,5\end{array}$ & $\begin{array}{r}11913 \\
3,0\end{array}$ & $\begin{array}{r}2460 \\
0,6\end{array}$ & $\begin{array}{l}- \\
-\end{array}$ & $\begin{array}{r}5203 \\
1,3\end{array}$ & $\begin{array}{r}133184 \\
33,4\end{array}$ \\
\hline BMLF & $\begin{array}{l}\text { in } 1000 \mathrm{~S} \\
\text { in } \%\end{array}$ & $\begin{array}{r}531007 \\
100,0\end{array}$ & $\begin{array}{r}4934 \\
0,9\end{array}$ & $\begin{array}{r}468554 \\
88,3\end{array}$ & $\begin{array}{r}29982 \\
5,6\end{array}$ & - & - & - & - & $\begin{array}{r}19288 \\
3,6\end{array}$ & $\begin{array}{r}8249 \\
1,6\end{array}$ & - & - & - & - \\
\hline BMWA & $\begin{array}{l}\text { in } 1000 \mathrm{~S} \\
\text { in } \%\end{array}$ & $\begin{array}{r}884603 \\
100,0\end{array}$ & - & $\begin{array}{r}7478 \\
0,8\end{array}$ & $\begin{array}{r}664901 \\
75,3\end{array}$ & $\begin{array}{r}57366 \\
6,5\end{array}$ & $\begin{array}{r}48109 \\
5,4\end{array}$ & - & $\begin{array}{r}87023 \\
9,8\end{array}$ & $\begin{array}{r}1943 \\
0,2\end{array}$ & $\begin{array}{r}10145 \\
1,1\end{array}$ & $\begin{array}{l}813 \\
0,1\end{array}$ & - & - & $\begin{array}{r}6825 \\
0,8\end{array}$ \\
\hline INSGESAMT & $\begin{array}{l}\text { in } 1000 \mathrm{~S} \\
\text { in } \%\end{array}$ & $\begin{array}{r}16385606 \\
100,0\end{array}$ & $\begin{array}{r}892135 \\
5,4\end{array}$ & $\begin{array}{r}729431 \\
4,5\end{array}$ & $\begin{array}{r}2287897 \\
14,0\end{array}$ & $\begin{array}{r}275574 \\
1,7\end{array}$ & $\begin{array}{r}431066 \\
2,6\end{array}$ & $\begin{array}{r}232540 \\
1,4\end{array}$ & $\begin{array}{r}3573892 \\
21,8\end{array}$ & $\begin{array}{r}1145878 \\
7,0\end{array}$ & $\begin{array}{r}594146 \\
3,6\end{array}$ & $\begin{array}{r}96477 \\
0,6\end{array}$ & $\begin{array}{l}790 \\
0,0\end{array}$ & $\begin{array}{r}157025 \\
1,0\end{array}$ & $\begin{array}{r}5968755 \\
36,4\end{array}$ \\
\hline Stand: April 20 & & & & & & & & & & & Quel & : Statistik A & ustria (Bundes & anstalt Statist & k Österreich) \\
\hline
\end{tabular}


Tabelle 6: Ausgaben des Bundes 1999 für Forschung und Forschungsförderung nach sozio-ökonomischen Zielsetzungen (ÖSTAT-Systematik) und Ressorts Aufgliederung der Jahreswerte $1999^{1}$ ) aus der Beilage T des Amtsbehelfes zum Bundesfinanzgesetz 2001 (Teil a und Teil b)

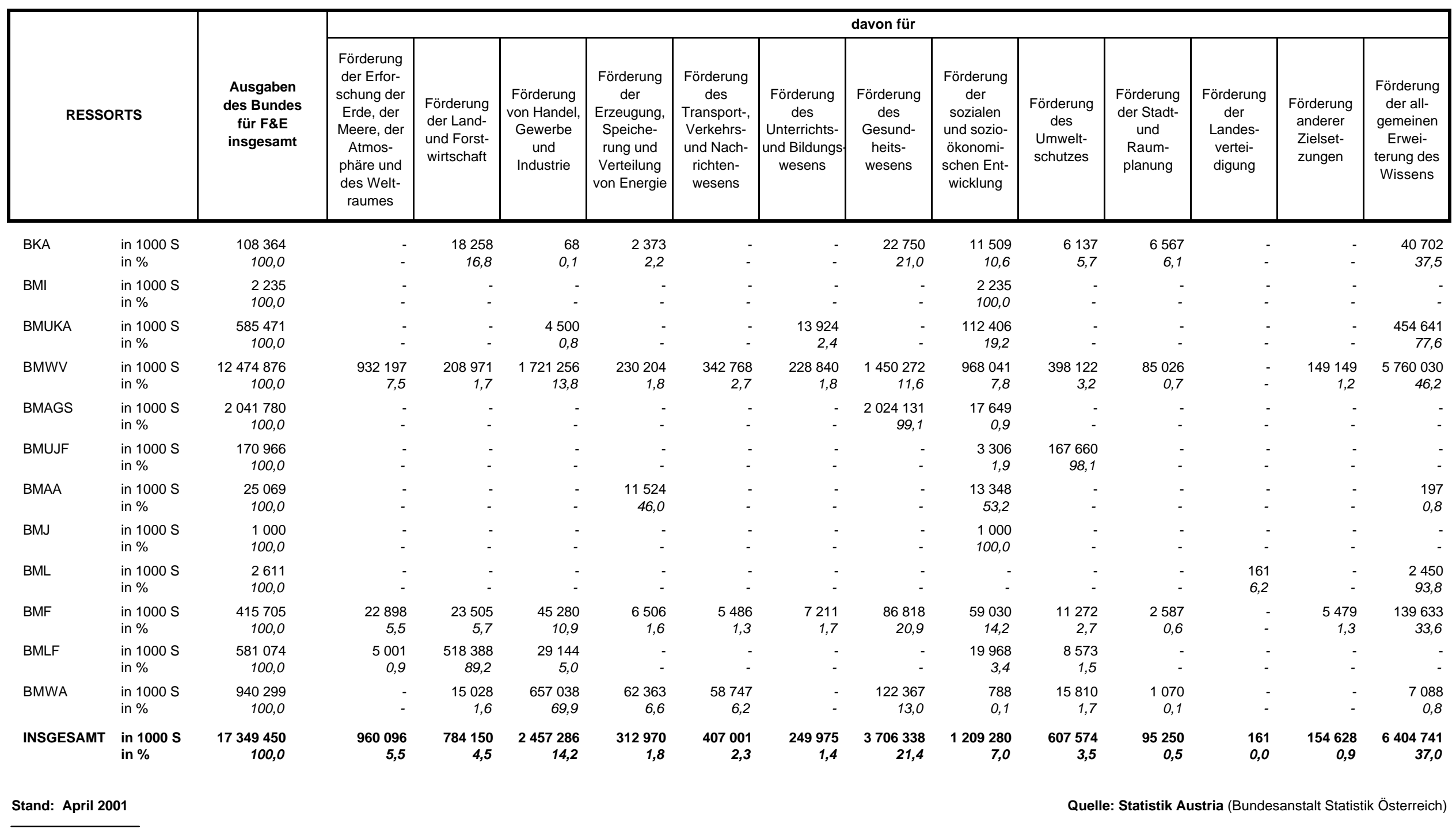


Tabelle 7: $\quad$ Ausgaben des Bundes 2000 für Forschung und Forschungsförderung nach sozio-ökonomischen Zielsetzungen (ÖSTAT-Systematik) und Ressorts

Aufgliederung der Jahreswerte $2000^{1}$ ) aus der Beilage T des Amtsbehelfes zum Bundesfinanzgesetz 2001 (Teil a und Teil b)

\begin{tabular}{|c|c|c|c|c|c|c|c|c|c|c|c|c|c|c|c|}
\hline \multirow{2}{*}{\multicolumn{2}{|c|}{ RESSORTS }} & \multirow[b]{2}{*}{$\begin{array}{c}\text { Ausgaben } \\
\text { des Bundes } \\
\text { für F\&E } \\
\text { insgesamt }\end{array}$} & \multicolumn{13}{|c|}{ davon für } \\
\hline & & & $\begin{array}{c}\text { Förderung } \\
\text { der Erfor- } \\
\text { schung der } \\
\text { Erde, der } \\
\text { Meere, der } \\
\text { Atmos- } \\
\text { phäre und } \\
\text { des Welt- } \\
\text { raumes }\end{array}$ & $\begin{array}{l}\text { Förderung } \\
\text { der Land- } \\
\text { und Forst- } \\
\text { wirtschaft }\end{array}$ & $\begin{array}{c}\text { Förderung } \\
\text { von Handel, } \\
\text { Gewerbe } \\
\text { und } \\
\text { Industrie }\end{array}$ & \begin{tabular}{|c|} 
Förderung \\
der \\
Erzeugung, \\
Speiche- \\
rung und \\
Verteilung \\
von Energie
\end{tabular} & \begin{tabular}{|c|} 
Förderung \\
des \\
Transport-, \\
Verkehrs- \\
und Nach- \\
richten- \\
wesens
\end{tabular} & \begin{tabular}{|c|} 
Förderung \\
des \\
Unterrichts- \\
und Bildungs \\
wesens
\end{tabular} & $\begin{array}{c}\text { Förderung } \\
\text { des } \\
\text { Gesund- } \\
\text { heits- } \\
\text { wesens }\end{array}$ & $\begin{array}{l}\text { Förderung } \\
\text { der } \\
\text { sozialen } \\
\text { und sozio- } \\
\text { ökonomi- } \\
\text { schen Ent- } \\
\text { wicklung }\end{array}$ & $\begin{array}{l}\text { Förderung } \\
\text { des } \\
\text { Umwelt- } \\
\text { schutzes }\end{array}$ & $\begin{array}{l}\text { Förderung } \\
\text { der Stadt- } \\
\text { und } \\
\text { Raum- } \\
\text { planung }\end{array}$ & $\begin{array}{l}\text { Förderung } \\
\text { der } \\
\text { Landes- } \\
\text { vertei- } \\
\text { digung }\end{array}$ & $\begin{array}{l}\text { Förderung } \\
\text { anderer } \\
\text { Zielset- } \\
\text { zungen }\end{array}$ & $\begin{array}{l}\text { Förderung } \\
\text { der all- } \\
\text { gemeinen } \\
\text { Erwei- } \\
\text { terung des } \\
\text { Wissens }\end{array}$ \\
\hline BKA & $\begin{array}{l}\text { in } 1000 \mathrm{~S} \\
\text { in } \%\end{array}$ & $\begin{array}{r}77907 \\
100,0\end{array}$ & - & $\begin{array}{r}4644 \\
6,0\end{array}$ & $\begin{array}{r}74 \\
0,1\end{array}$ & $\begin{array}{r}4545 \\
5,8\end{array}$ & - & - & $\begin{array}{r}6266 \\
8,0\end{array}$ & $\begin{array}{r}14696 \\
18,9\end{array}$ & $\begin{array}{r}1386 \\
1,8\end{array}$ & $\begin{array}{r}6876 \\
8,8\end{array}$ & - & - & $\begin{array}{r}39420 \\
50,6\end{array}$ \\
\hline $\mathrm{BMI}$ & $\begin{array}{l}\text { in } 1000 \mathrm{~S} \\
\text { in } \%\end{array}$ & $\begin{array}{l}1994 \\
100,0\end{array}$ & - & - & - & - & - & - & - & $\begin{array}{l}1994 \\
100,0\end{array}$ & - & - & - & - & - \\
\hline BMBWK & $\begin{array}{l}\text { in } 1000 \mathrm{~S} \\
\text { in } \%\end{array}$ & $\begin{array}{r}12546775 \\
100,0\end{array}$ & $\begin{array}{r}693101 \\
5,5\end{array}$ & $\begin{array}{r}181658 \\
1,4\end{array}$ & $\begin{array}{r}1337695 \\
10,7\end{array}$ & $\begin{array}{r}185728 \\
1,5\end{array}$ & $\begin{array}{r}139749 \\
1,1\end{array}$ & $\begin{array}{r}221185 \\
1,8\end{array}$ & $\begin{array}{r}2818095 \\
22,5\end{array}$ & $\begin{array}{r}987538 \\
7,9\end{array}$ & $\begin{array}{r}308613 \\
2,5\end{array}$ & $\begin{array}{r}77087 \\
0,6\end{array}$ & $\begin{array}{r}598 \\
0,0\end{array}$ & $\begin{array}{r}137709 \\
1,1\end{array}$ & $\begin{array}{r}5458019 \\
43,4\end{array}$ \\
\hline BMSG & $\begin{array}{l}\text { in } 1000 \mathrm{~S} \\
\text { in } \%\end{array}$ & $\begin{array}{r}483920 \\
100,0\end{array}$ & - & $\begin{array}{r}14202 \\
2,9\end{array}$ & - & - & - & - & $\begin{array}{r}443100 \\
91,6\end{array}$ & $\begin{array}{r}17684 \\
3,7\end{array}$ & $\begin{array}{r}8934 \\
1,8\end{array}$ & - & - & - & - \\
\hline BMAA & $\begin{array}{l}\text { in } 1000 \mathrm{~S} \\
\text { in } \%\end{array}$ & $\begin{array}{r}27570 \\
100,0\end{array}$ & - & - & - & $\begin{array}{r}12688 \\
46,0\end{array}$ & - & - & - & $\begin{array}{r}14642 \\
53,1\end{array}$ & - & - & - & - & $\begin{array}{r}240 \\
0,9\end{array}$ \\
\hline BMJ & $\begin{array}{l}\text { in } 1000 \mathrm{~S} \\
\text { in } \%\end{array}$ & $\begin{array}{r}850 \\
100,0\end{array}$ & - & - & - & - & - & - & - & $\begin{array}{r}850 \\
100,0\end{array}$ & - & - & - & - & - \\
\hline BML & $\begin{array}{l}\text { in } 1000 \mathrm{~S} \\
\text { in } \%\end{array}$ & $\begin{array}{l}3267 \\
100,0\end{array}$ & - & - & - & - & - & - & - & - & - & - & $\begin{array}{r}550 \\
16,8\end{array}$ & - & $\begin{array}{r}2717 \\
83,2\end{array}$ \\
\hline BMF & $\begin{array}{l}\text { in } 1000 \mathrm{~S} \\
\text { in } \%\end{array}$ & $\begin{array}{r}429933 \\
100,0\end{array}$ & $\begin{array}{r}23720 \\
5,5\end{array}$ & $\begin{array}{r}24087 \\
5,6\end{array}$ & $\begin{array}{r}47087 \\
11,0\end{array}$ & $\begin{array}{r}6760 \\
1,6\end{array}$ & $\begin{array}{r}5717 \\
1,3\end{array}$ & $\begin{array}{r}7532 \\
1,8\end{array}$ & $\begin{array}{r}90138 \\
21,0\end{array}$ & $\begin{array}{r}60202 \\
14,0\end{array}$ & $\begin{array}{r}11723 \\
2,7\end{array}$ & $\begin{array}{r}2699 \\
0,6\end{array}$ & - & $\begin{array}{r}5693 \\
1,3\end{array}$ & $\begin{array}{r}144575 \\
33,6\end{array}$ \\
\hline BMLFUW $^{2}$ ) & $\begin{array}{l}\text { in } 1000 \mathrm{~S} \\
\text { in } \%\end{array}$ & $\begin{array}{r}731108 \\
100,0\end{array}$ & $\begin{array}{r}5142 \\
0,7\end{array}$ & $\begin{array}{r}485020 \\
66,3\end{array}$ & $\begin{array}{r}28168 \\
3,9\end{array}$ & - & - & - & - & $\begin{array}{r}21350 \\
2,9\end{array}$ & $\begin{array}{r}191428 \\
26,2\end{array}$ & - & - & - & $\begin{array}{l}- \\
-\end{array}$ \\
\hline BMWA & $\begin{array}{l}\text { in } 1000 \mathrm{~S} \\
\text { in } \%\end{array}$ & $\begin{array}{r}89063 \\
100,0\end{array}$ & - & - & $\begin{array}{r}69436 \\
78,0\end{array}$ & - & $\begin{array}{r}429 \\
0,5\end{array}$ & - & - & $\begin{array}{r}12919 \\
14,5\end{array}$ & $\begin{array}{r}856 \\
1,0\end{array}$ & $\begin{array}{r}754 \\
0,8\end{array}$ & - & - & $\begin{array}{r}4669 \\
5,2\end{array}$ \\
\hline BMVIT & $\begin{array}{l}\text { in } 1000 \mathrm{~S} \\
\text { in } \%\end{array}$ & $\begin{array}{r}2075895 \\
100,0\end{array}$ & $\begin{array}{r}174188 \\
8,4\end{array}$ & $\begin{array}{r}17946 \\
0,9\end{array}$ & $\begin{array}{r}746509 \\
35,9\end{array}$ & $\begin{array}{r}46786 \\
2,3\end{array}$ & $\begin{array}{r}290750 \\
14,0\end{array}$ & $\begin{array}{r}4997 \\
0,2\end{array}$ & $\begin{array}{r}225734 \\
10,9\end{array}$ & $\begin{array}{r}22129 \\
1,1\end{array}$ & $\begin{array}{r}101647 \\
4,9\end{array}$ & $\begin{array}{r}5945 \\
0,3\end{array}$ & $\begin{array}{r}2468 \\
0,1\end{array}$ & $\begin{array}{r}1518 \\
0,1\end{array}$ & $\begin{array}{r}435278 \\
20,9\end{array}$ \\
\hline BMOLS & $\begin{array}{l}\text { in } 1000 \mathrm{~S} \\
\text { in } \%\end{array}$ & - & - & - & - & - & - & - & - & - & - & - & - & - & - \\
\hline INSGESAMT & $\begin{array}{l}\text { in } 1000 \mathrm{~S} \\
\text { in } \%\end{array}$ & $\begin{array}{r}16468282 \\
100,0\end{array}$ & $\begin{array}{r}896151 \\
5,4\end{array}$ & $\begin{array}{r}727557 \\
4,4\end{array}$ & $\begin{array}{r}2228969 \\
13,5\end{array}$ & $\begin{array}{r}256507 \\
1,6\end{array}$ & $\begin{array}{r}436645 \\
2,7\end{array}$ & $\begin{array}{r}233714 \\
1,4\end{array}$ & $\begin{array}{r}3583333 \\
21,8\end{array}$ & $\begin{array}{r}1154004 \\
7,0\end{array}$ & $\begin{array}{r}624587 \\
3,8\end{array}$ & $\begin{array}{r}93361 \\
0,6\end{array}$ & $\begin{array}{r}3616 \\
0,0\end{array}$ & $\begin{array}{r}144920 \\
0,9\end{array}$ & $\begin{array}{r}6084918 \\
36,9\end{array}$ \\
\hline
\end{tabular}

Stand: April 2001

1) Bundesvoranschlag.

$\left.{ }^{2}\right)$ Einschließlich der im Budgetkapitel 18 veranschlagten Ausgaben für Forschung und Forschungsförderung im Bereich Umwelt. 
Tabelle 8: Ausgaben des Bundes 2001 für Forschung und Forschungsförderung nach sozio-ökonomischen Zielsetzungen (ÖSTAT-Systematik) und Ressorts Ohne die im BFG 2001 rücklagefähig veranschlagten Mittel der "Forschungs- und Technologieoffensive" (VA-Ansatz 1/5182; 7 Mrd. Schilling)

Aufgliederung der Jahreswerte $200{ }^{1}{ }^{\text {}}$ ) aus der Beilage T des Amtsbehelfes zum Bundesfinanzgesetz 2001 (Teil a und Teil b)

\begin{tabular}{|c|c|c|c|c|c|c|c|c|c|c|c|c|c|c|c|}
\hline \multirow{2}{*}{\multicolumn{2}{|c|}{ RESSORTS }} & \multirow[b]{2}{*}{$\begin{array}{c}\text { Ausgaben } \\
\text { des Bundes } \\
\text { für F\&E } \\
\text { insgesamt }\end{array}$} & \multicolumn{13}{|c|}{ davon für } \\
\hline & & & $\begin{array}{c}\text { Förderung } \\
\text { der Erfor- } \\
\text { schung der } \\
\text { Erde, der } \\
\text { Meere, der } \\
\text { Atmos- } \\
\text { phäre und } \\
\text { des Welt- } \\
\text { raumes }\end{array}$ & $\begin{array}{l}\text { Förderung } \\
\text { der Land- } \\
\text { und Forst- } \\
\text { wirtschaft }\end{array}$ & $\begin{array}{c}\text { Förderung } \\
\text { von Handel, } \\
\text { Gewerbe } \\
\text { und } \\
\text { Industrie }\end{array}$ & $\begin{array}{l}\text { Förderung } \\
\text { der } \\
\text { Erzeugung, } \\
\text { Speiche- } \\
\text { rung und } \\
\text { Verteilung } \\
\text { von Energie }\end{array}$ & \begin{tabular}{|c|} 
Förderung \\
des Transport \\
, \\
Verkehrs- \\
und Nach- \\
richten- \\
wesens
\end{tabular} & $\begin{array}{c}\text { Förderung } \\
\text { des } \\
\text { Unterrichts- } \\
\text { und Bildungs- } \\
\text { wesens }\end{array}$ & $\begin{array}{l}\text { Förderung } \\
\text { des } \\
\text { Gesund- } \\
\text { heits- } \\
\text { wesens }\end{array}$ & $\begin{array}{c}\text { Förderung } \\
\text { der } \\
\text { sozialen } \\
\text { und sozio- } \\
\text { ökonomi- } \\
\text { schen Ent- } \\
\text { wicklung }\end{array}$ & $\begin{array}{c}\text { Förderung } \\
\text { des } \\
\text { Umwelt- } \\
\text { schutzes }\end{array}$ & $\begin{array}{c}\text { Förderung } \\
\text { der Stadt- } \\
\text { und } \\
\text { Raum- } \\
\text { planung }\end{array}$ & $\begin{array}{c}\text { Förderung } \\
\text { der } \\
\text { Landes- } \\
\text { vertei- } \\
\text { digung }\end{array}$ & $\begin{array}{l}\text { Förderung } \\
\text { anderer } \\
\text { Zielset- } \\
\text { zungen }\end{array}$ & $\begin{array}{l}\text { Förderung } \\
\text { der all- } \\
\text { gemeinen } \\
\text { Erwei- } \\
\text { terung des } \\
\text { Wissens }\end{array}$ \\
\hline BKA & $\begin{array}{l}\text { in } 1000 \mathrm{~S} \\
\text { in } \%\end{array}$ & $\begin{array}{r}70521 \\
100,0\end{array}$ & - & - & $\begin{array}{r}75 \\
0,1\end{array}$ & $\begin{array}{r}2900 \\
4,1\end{array}$ & - & - & - & $\begin{array}{r}6686 \\
9,5\end{array}$ & - & $\begin{array}{r}6254 \\
8,9\end{array}$ & - & 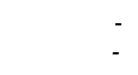 & $\begin{array}{r}54606 \\
77,4\end{array}$ \\
\hline BMI & $\begin{array}{l}\text { in } 1000 \mathrm{~S} \\
\text { in } \%\end{array}$ & $\begin{array}{l}1994 \\
100,0\end{array}$ & - & - & - & - & - & - & - & $\begin{array}{l}1994 \\
100,0\end{array}$ & - & - & - & - & - \\
\hline BMBWK & $\begin{array}{l}\text { in } 1000 \mathrm{~S} \\
\text { in } \%\end{array}$ & $\begin{array}{r}12957420 \\
100,0\end{array}$ & $\begin{array}{r}626412 \\
4,8\end{array}$ & $\begin{array}{r}187062 \\
1,4\end{array}$ & $\begin{array}{r}1285217 \\
9,9\end{array}$ & $\begin{array}{r}189293 \\
1,5\end{array}$ & $\begin{array}{r}138626 \\
1,1\end{array}$ & $\begin{array}{r}235307 \\
1,8\end{array}$ & $\begin{array}{r}3474805 \\
26,8\end{array}$ & $\begin{array}{r}1002155 \\
7,7\end{array}$ & $\begin{array}{r}297166 \\
2,3\end{array}$ & $\begin{array}{r}77743 \\
0,6\end{array}$ & - & $\begin{array}{r}144429 \\
1,1\end{array}$ & $\begin{array}{r}5299205 \\
41,0\end{array}$ \\
\hline BMSG & $\begin{array}{l}\text { in } 1000 \mathrm{~S} \\
\text { in } \%\end{array}$ & $\begin{array}{r}145958 \\
100,0\end{array}$ & - & $\begin{array}{r}21149 \\
14,5\end{array}$ & - & - & - & - & $\begin{array}{c}85818 \\
58,8\end{array}$ & $\begin{array}{r}26122 \\
17,9\end{array}$ & $\begin{array}{r}12869 \\
8,8\end{array}$ & - & - & - & - \\
\hline BMAA & $\begin{array}{l}\text { in } 1000 \mathrm{~S} \\
\text { in } \%\end{array}$ & $\begin{array}{r}27570 \\
100,0\end{array}$ & - & - & - & $\begin{array}{r}12688 \\
46,0\end{array}$ & - & - & - & $\begin{array}{r}14642 \\
53,1\end{array}$ & - & - & - & - & $\begin{array}{r}240 \\
0,9\end{array}$ \\
\hline BMJ & $\begin{array}{l}\text { in } 1000 \mathrm{~S} \\
\text { in } \%\end{array}$ & $\begin{array}{r}850 \\
100,0\end{array}$ & - & - & - & - & - & - & - & $\begin{array}{r}850 \\
100,0\end{array}$ & - & - & - & - & - \\
\hline BML & $\begin{array}{l}\text { in } 1000 \mathrm{~S} \\
\text { in } \%\end{array}$ & $\begin{array}{l}3547 \\
100,0\end{array}$ & - & - & - & - & - & - & - & - & - & - & $\begin{array}{r}960 \\
27,1\end{array}$ & - & $\begin{array}{r}2587 \\
72,9\end{array}$ \\
\hline BMF & $\begin{array}{l}\text { in } 1000 \mathrm{~S} \\
\text { in } \%\end{array}$ & $\begin{array}{r}442947 \\
100,0\end{array}$ & $\begin{array}{r}24204 \\
5,5\end{array}$ & $\begin{array}{r}23058 \\
5,2\end{array}$ & $\begin{array}{r}47888 \\
10,8\end{array}$ & $\begin{array}{r}6996 \\
1,6\end{array}$ & $\begin{array}{r}5865 \\
1,3\end{array}$ & $\begin{array}{r}7768 \\
1,8\end{array}$ & $\begin{array}{r}93255 \\
21,1\end{array}$ & $\begin{array}{r}64198 \\
14,5\end{array}$ & $\begin{array}{r}12019 \\
2,7\end{array}$ & $\begin{array}{r}2785 \\
0,6\end{array}$ & - & $\begin{array}{r}5891 \\
1,3\end{array}$ & $\begin{array}{r}149020 \\
33,6\end{array}$ \\
\hline BMLFUW & $\begin{array}{l}\text { in } 1000 \mathrm{~S} \\
\text { in } \%\end{array}$ & $\begin{array}{r}725487 \\
100,0\end{array}$ & $\begin{array}{r}5067 \\
0,7\end{array}$ & $\begin{array}{r}486200 \\
67,0\end{array}$ & $\begin{array}{r}28955 \\
4,0\end{array}$ & - & - & - & - & $\begin{array}{r}21350 \\
2,9\end{array}$ & $\begin{array}{r}183915 \\
25,4\end{array}$ & - & - & - & - \\
\hline BMWA & $\begin{array}{l}\text { in } 1000 \mathrm{~S} \\
\text { in } \%\end{array}$ & $\begin{array}{r}93855 \\
100,0\end{array}$ & - & - & $\begin{array}{r}70356 \\
75,0\end{array}$ & $\begin{array}{r}3 \\
0,0\end{array}$ & $\begin{array}{r}14 \\
0,0\end{array}$ & - & $\begin{array}{r}6 \\
0,0\end{array}$ & $\begin{array}{r}17317 \\
18,5\end{array}$ & $\begin{array}{r}757 \\
0,8\end{array}$ & $\begin{array}{r}782 \\
0,8\end{array}$ & - & - & $\begin{array}{r}4620 \\
4,9\end{array}$ \\
\hline BMVIT & $\begin{array}{l}\text { in } 1000 \mathrm{~S} \\
\text { in } \%\end{array}$ & $\begin{array}{r}2571379 \\
100,0\end{array}$ & $\begin{array}{r}208836 \\
8,1\end{array}$ & $\begin{array}{r}21105 \\
0,8\end{array}$ & $\begin{array}{r}1027108 \\
40,0\end{array}$ & $\begin{array}{r}49149 \\
1,9\end{array}$ & $\begin{array}{r}309104 \\
12,0\end{array}$ & $\begin{array}{r}6153 \\
0,2\end{array}$ & $\begin{array}{r}252776 \\
9,8\end{array}$ & $\begin{array}{r}25979 \\
1,0\end{array}$ & $\begin{array}{r}115765 \\
4,5\end{array}$ & $\begin{array}{r}7753 \\
0,3\end{array}$ & $\begin{array}{r}3305 \\
0,1\end{array}$ & $\begin{array}{r}1709 \\
0,1\end{array}$ & $\begin{array}{r}542637 \\
21,2\end{array}$ \\
\hline BMOLS & $\begin{array}{l}\text { in } 1000 \mathrm{~S} \\
\text { in } \%\end{array}$ & - & - & - & - & - & - & - & $\begin{array}{l}- \\
-\end{array}$ & - & - & - & $\begin{array}{l}- \\
-\end{array}$ & $\begin{array}{l}- \\
-\end{array}$ & - \\
\hline INSGESAMT & $\begin{array}{l}\text { in } 1000 \mathrm{~S} \\
\text { in } \%\end{array}$ & $\begin{array}{r}17041528 \\
100,0\end{array}$ & $\begin{array}{r}864519 \\
5,1\end{array}$ & $\begin{array}{r}738574 \\
4,3\end{array}$ & $\begin{array}{r}2459599 \\
14,4\end{array}$ & $\begin{array}{r}261029 \\
1,5\end{array}$ & $\begin{array}{r}453609 \\
2,7\end{array}$ & $\begin{array}{r}249228 \\
1,5\end{array}$ & $\begin{array}{r}3906660 \\
22,9\end{array}$ & $\begin{array}{r}1181293 \\
6,9\end{array}$ & $\begin{array}{r}622491 \\
3,7\end{array}$ & $\begin{array}{r}95317 \\
0,6\end{array}$ & $\begin{array}{r}4265 \\
0,0\end{array}$ & $\begin{array}{r}152029 \\
0,9\end{array}$ & $\begin{array}{r}6052915 \\
35,5\end{array}$ \\
\hline
\end{tabular}


Tabelle 9: $\quad$ Forschungsförderungen und Forschungsaufträge des Bundes 1999 nach Förderungsempfängern bzw. Auftragnehmern (gegliedert nach volkswirtschaftlichen Sektoren/Bereichen) und vergebenden Ressorts

Auswertung der Faktendokumentation der Bundesdienststellen für 1999 (Stand: Dezember 2000) einschließlich "große" Globalförderungen ${ }^{1}$ )

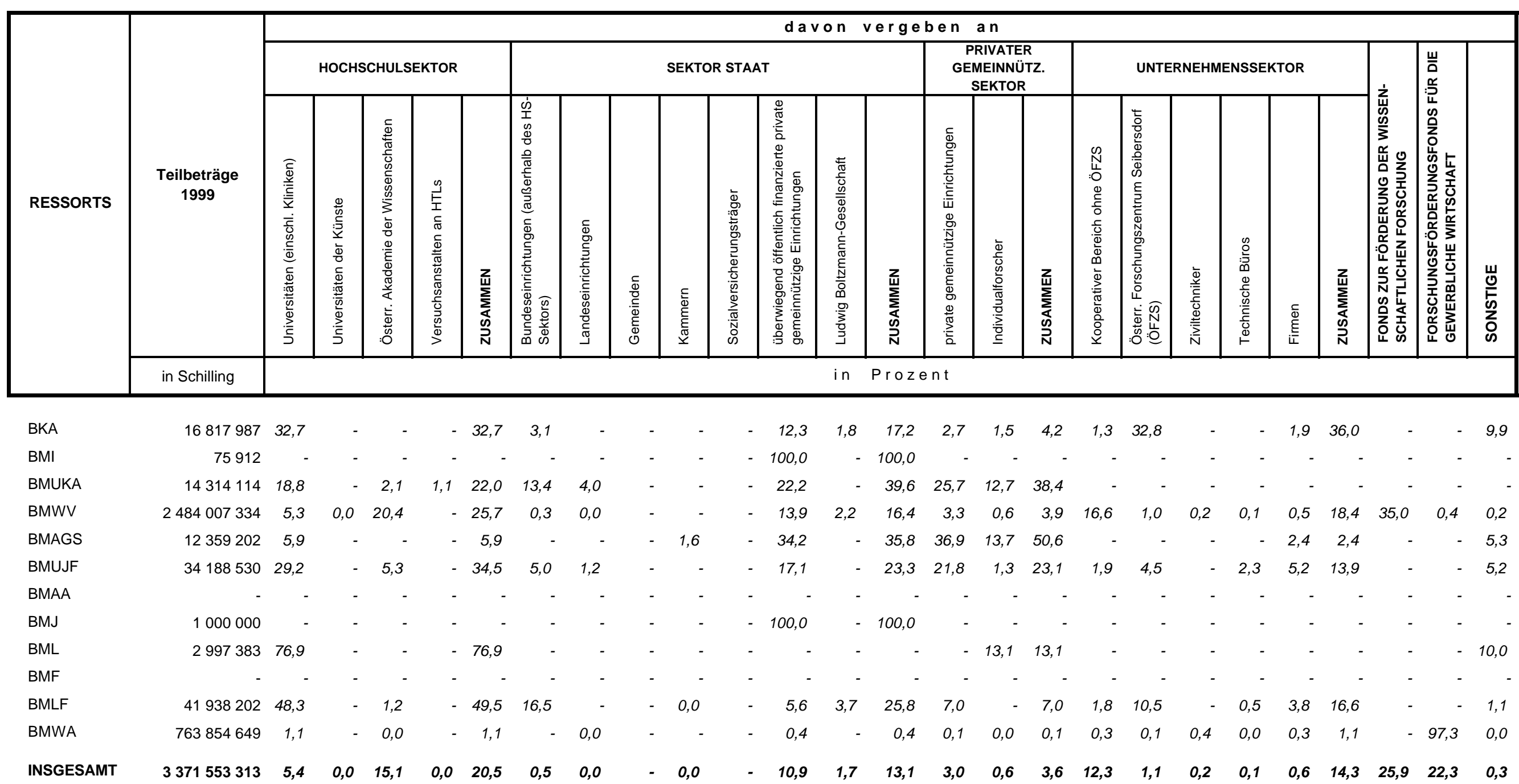


Auswertung der Faktendokumentation der Bundesdienststellen für 1999 (Stand: Dezember 2000) ohne "große" Globalförderungen ${ }^{1}$ )

\begin{tabular}{|c|c|c|c|c|c|c|c|c|c|c|c|c|c|c|c|c|c|c|c|c|c|c|c|c|c|c|}
\hline \multirow{3}{*}{ RESSORTS } & \multirow[b]{2}{*}{$\begin{array}{c}\text { Teilbeträge } \\
1999\end{array}$} & \multicolumn{5}{|c|}{ HOCHSCHULSEKTOR } & \multicolumn{8}{|c|}{ SEKTOR STAAT } & \multicolumn{3}{|c|}{$\begin{array}{c}\text { PRIVATER } \\
\text { GEMEINNÜTZ. } \\
\text { SEKTOR }\end{array}$} & \multicolumn{6}{|c|}{ UNTERNEHMENSSEKTOR } & \multirow[b]{2}{*}{ 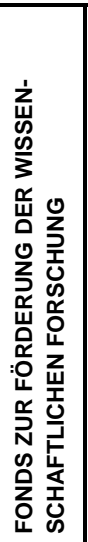 } & \multirow[b]{2}{*}{ 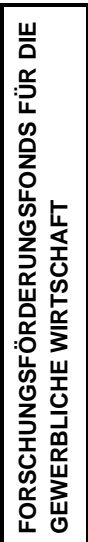 } & \multirow[b]{2}{*}{ 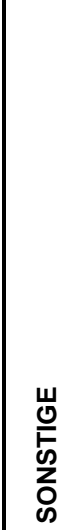 } \\
\hline & & 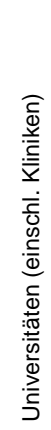 & 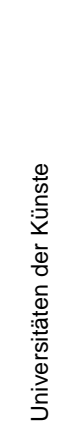 & 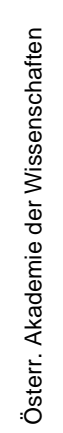 & 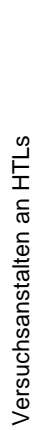 & 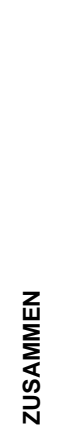 & 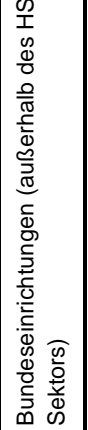 & 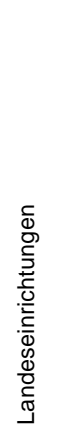 & 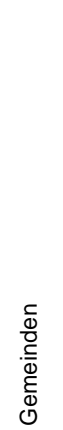 & 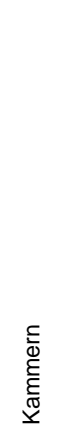 & 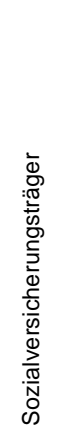 & 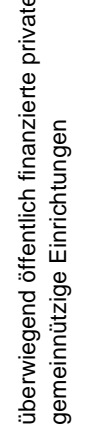 & 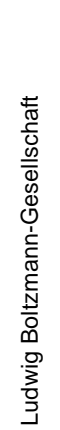 & 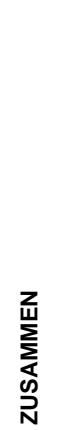 & 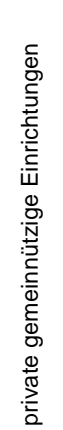 & 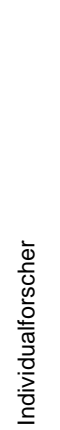 & 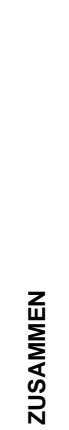 & 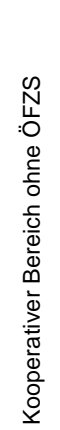 & 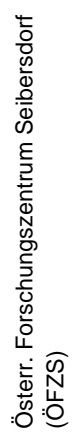 & 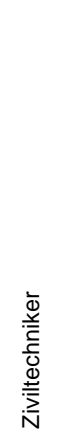 & 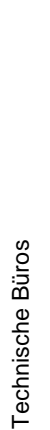 & 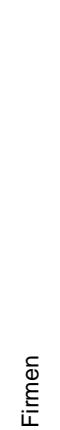 & 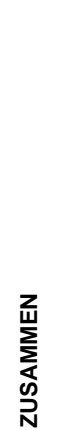 & & & \\
\hline & in Schilling & & & & & & & & & & & & & & & & & & & & & & & & & \\
\hline
\end{tabular}

\begin{tabular}{|c|c|c|c|c|c|c|c|c|c|c|c|c|c|c|c|c|c|c|c|c|c|c|c|c|c|c|}
\hline BKA & 16817987 & 32,7 & - & - & - & 32,7 & 3,1 & - & - & - & - & 12,3 & 1,8 & 17,2 & 2,7 & 1,5 & 4,2 & 1,3 & 32,8 & - & - & 1,9 & 36,0 & - & - & 9,9 \\
\hline BMI & 75912 & & - & - & . & - & - & - & - & - & - & 100,0 & - & 100,0 & - & - & - & - & - & - & - & - & - & - & - & \\
\hline BMUKA & 14314114 & 18,8 & - & 2,1 & 1,1 & 22,0 & 13,4 & 4,0 & - & - & - & 22,2 & - & 39,6 & 25,7 & 12,7 & 38,4 & - & - & - & - & - & - & - & - & \\
\hline BMWV & 635628684 & 20,7 & 0,1 & 14,9 & - & 35,7 & 1,1 & 0,0 & - & - & - & 27,1 & 0,9 & 29,1 & 12,8 & 2,2 & 15,0 & 8,7 & 3,8 & 0,7 & 0,3 & 2,1 & 15,6 & 2,2 & 1,7 & 0,7 \\
\hline BMAGS & 12359202 & 5,9 & - & - & . & 5,9 & - & - & - & 1,6 & - & 34,2 & - & 35,8 & 36,9 & 13,7 & 50,6 & - & - & - & - & 2,4 & 2,4 & - & - & 5,3 \\
\hline BMUJF & 34188530 & 29,2 & - & 5,3 & . & 34,5 & 5,0 & 1,2 & - & - & - & 17,1 & - & 23,3 & 21,8 & 1,3 & 23,1 & 1,9 & 4,5 & - & 2,3 & 5,2 & 13,9 & - & - & 5,2 \\
\hline BMAA & - & - & - & - & - & - & - & - & - & - & - & - & - & - & - & - & - & - & - & - & - & - & - & - & - & \\
\hline BMJ & 1000000 & - & - & - & - & - & - & - & - & - & - & 100,0 & - & 100,0 & - & - & - & - & - & - & - & - & - & - & - & \\
\hline BML & 2997383 & 76,9 & - & - & - & 76,9 & - & - & - & - & - & - & - & - & - & 13,1 & 13,1 & - & - & - & - & - & - & - & - & 10,0 \\
\hline BMF & - & - & - & - & - & - & - & - & - & - & - & - & - & - & - & - & - & - & - & - & - & - & - & - & - & \\
\hline BMLF & 41938202 & 48,3 & - & 1,2 & - & 49,5 & 16,5 & - & - & 0,0 & - & 5,6 & 3,7 & 25,8 & 7,0 & - & 7,0 & 1,8 & 10,5 & - & 0,5 & 3,8 & 16,6 & - & - & 1,1 \\
\hline BMWA & 21563149 & 39,9 & - & 0,8 & - & 40,7 & - & 0,2 & - & - & - & 15,2 & - & 15,4 & 4,3 & 1,3 & 5,6 & 8,9 & 3,5 & 13,1 & 1,0 & 10,9 & 37,4 & - & - & 0,9 \\
\hline INSGESAMT & 780883163 & 23,3 & 0,0 & 12,5 & 0,0 & 35,8 & 2,3 & 0,2 & - & 0,0 & - & 24,8 & 1,0 & 28,3 & 13,0 & 2,4 & 15,4 & 7,6 & 4,7 & 0,9 & 0,4 & 2,5 & 16,1 & 1,8 & 1,4 & \\
\hline
\end{tabular}

Stand: April 2001

1) d.h. ohne Globalförderungen für: Fonds zur Förderung der wissenschaftlichen Forschung, Forschungsförderungsfonds für die gewerbliche Wirtschaft, Ludwig Boltzmann-Gesellschaft, Österreichische Akademie der Wissenschaften, Österreichisches Forschungszentrum Seibersdorf (insgesamt 2590670150 Schilling). Die in der Faktendokumentation 1999 nicht enthaltene Globalförderung seitens des BMWA an den FFF wurde unter Heranziehung des Bundesrechnungsabschlusses 1999 ermittelt. 
Tabelle 11: Forschungsförderungen und Forschungsaufträge des Bundes 1999 nach sozio-ökonomischen Zielsetzungen und vergebenden Ressorts

Auswertung der Faktendokumentation der Bundesdienststellen für 1999 (Stand: Dezember 2000) nach sozio-ökonomischen Zielsetzungen (ÖSTAT-Klassifikation), einschließlich "große" Globalförderungen ${ }^{1}$ )

\begin{tabular}{|c|c|c|c|c|c|c|c|c|c|c|c|c|c|c|c|}
\hline \multirow{2}{*}{\multicolumn{2}{|c|}{ RESSORTS }} & \multirow[b]{2}{*}{$\begin{array}{c}\text { Teilbeträge } \\
1999\end{array}$} & \multicolumn{13}{|c|}{ davon für } \\
\hline & & & \begin{tabular}{|c|} 
Förderung der \\
Errorschung \\
der Erde, \\
der Meere, \\
der Atmosphäre \\
und des \\
Weltraumes
\end{tabular} & $\begin{array}{c}\text { Förderung der } \\
\text { Land- - und } \\
\text { Forstwirtschaft }\end{array}$ & $\begin{array}{c}\text { Förderung von } \\
\text { Handel, } \\
\text { Gewerbe und } \\
\text { Industrie }\end{array}$ & $\begin{array}{l}\text { Förderung der } \\
\text { Erzeugung, } \\
\text { Speicherung } \\
\text { und Verteilung } \\
\text { von Energie }\end{array}$ & $\begin{array}{c}\text { Förderung des } \\
\text { Transport-, } \\
\text { Verkehrs- und } \\
\text { Nachrichten- } \\
\text { wesens }\end{array}$ & $\begin{array}{l}\text { Förderung des } \\
\text { Unterrichts- } \\
\text { und Bildungs- } \\
\text { wesens }\end{array}$ & $\begin{array}{c}\text { Förderung des } \\
\text { Gesund- } \\
\text { heitswesesens }\end{array}$ & \begin{tabular}{|c|} 
Förderung der \\
sozialen und \\
sozio-önonomi- \\
schen Ent- \\
wicklung
\end{tabular} & $\begin{array}{c}\text { Förderung des } \\
\text { Umwelt- } \\
\text { schutzes }\end{array}$ & $\begin{array}{l}\text { Förderung der } \\
\text { Stadt- und } \\
\text { Raum- } \\
\text { planung }\end{array}$ & \begin{tabular}{|c|} 
Förderung der \\
Landesverteidi- \\
gung
\end{tabular} & $\begin{array}{l}\text { Förderung } \\
\text { anderer Ziel- } \\
\text { setzungen }\end{array}$ & $\begin{array}{l}\text { Förderung der } \\
\text { allgemeinen } \\
\text { Erweiterung } \\
\text { des Wissens }\end{array}$ \\
\hline BKA & $\begin{array}{l}\text { in Schilling } \\
\text { in } \%\end{array}$ & $\begin{array}{r}16817987 \\
100,0\end{array}$ & $\begin{array}{r}246656 \\
1,5\end{array}$ & $\begin{array}{r}1063313 \\
6,3\end{array}$ & $\begin{array}{r}86300 \\
0,5\end{array}$ & - & - & $\begin{array}{r}270000 \\
1,6\end{array}$ & $\begin{array}{r}3039975 \\
18,1\end{array}$ & $\begin{array}{r}2350012 \\
14,0\end{array}$ & $\begin{array}{r}7027935 \\
41,7\end{array}$ & - & - & - & $\begin{array}{r}2733796 \\
16,3\end{array}$ \\
\hline BMI & $\begin{array}{l}\text { in Schilling } \\
\text { in } \%\end{array}$ & $\begin{array}{r}75912 \\
100,0\end{array}$ & - & - & - & - & $\begin{array}{r}75912 \\
100,0\end{array}$ & - & - & - & - & - & - & - & - \\
\hline BMUKA & $\begin{array}{l}\text { in Schilling } \\
\text { in } \%\end{array}$ & $\begin{array}{r}14314114 \\
100,0\end{array}$ & - & - & - & - & - & $\begin{array}{r}3735550 \\
26,1\end{array}$ & - & $\begin{array}{r}1842140 \\
12,9\end{array}$ & - & - & - & - & $\begin{array}{r}8736424 \\
61,0\end{array}$ \\
\hline BMWV & $\begin{array}{l}\text { in Schilling } \\
\text { in \% }\end{array}$ & $\begin{array}{r}2484007334 \\
100,0\end{array}$ & $\begin{array}{r}195777677 \\
7,9\end{array}$ & $\begin{array}{r}32172580 \\
1,3\end{array}$ & $\begin{array}{r}286635452 \\
11,5\end{array}$ & $\begin{array}{r}53061341 \\
2,1\end{array}$ & $\begin{array}{r}75445020 \\
3,0\end{array}$ & $\begin{array}{r}27130140 \\
1,1\end{array}$ & $\begin{array}{r}380456628 \\
15,3\end{array}$ & $\begin{array}{r}213996882 \\
8,6\end{array}$ & $\begin{array}{r}154635043 \\
6,2\end{array}$ & $\begin{array}{r}18009751 \\
0,7\end{array}$ & - & $\begin{array}{r}6128343 \\
0,2\end{array}$ & $\begin{array}{r}1040558477 \\
42,1\end{array}$ \\
\hline BMAGS & $\begin{array}{l}\text { in Schilling } \\
\text { in \% }\end{array}$ & $\begin{array}{r}12359202 \\
100,0\end{array}$ & - & - & - & - & : & - & $\begin{array}{r}713000 \\
5,8\end{array}$ & $\begin{array}{r}9010271 \\
72,9\end{array}$ & - & - & - & - & $\begin{array}{r}2635931 \\
21,3\end{array}$ \\
\hline BMUJF & $\begin{array}{l}\text { in Schilling } \\
\text { in } \%\end{array}$ & $\begin{array}{r}34188530 \\
100,0\end{array}$ & $\begin{array}{r}2146173 \\
6,3\end{array}$ & $\begin{array}{r}690000 \\
2,0\end{array}$ & $\begin{array}{r}478977 \\
1,4\end{array}$ & $\begin{array}{r}2081473 \\
6,1\end{array}$ & : & - & $\begin{array}{r}2829967 \\
8,3\end{array}$ & $\begin{array}{r}8913654 \\
26,1\end{array}$ & $\begin{array}{r}11518499 \\
33,6\end{array}$ & $\begin{array}{r}2289236 \\
6,7\end{array}$ & - & - & $\begin{array}{r}3240551 \\
9,5\end{array}$ \\
\hline BMAA & $\begin{array}{l}\text { in Schilling } \\
\text { in \% }\end{array}$ & & - & - & - & - & : & - & & - & - & - & - & - & . \\
\hline BMJ & $\begin{array}{l}\text { in Schilling } \\
\text { in } \%\end{array}$ & $\begin{array}{r}1000000 \\
100,0\end{array}$ & - & - & - & - & - & - & 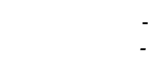 & $\begin{array}{r}1000000 \\
100,0\end{array}$ & - & - & - & - & . \\
\hline BML & $\begin{array}{l}\text { in Schilling } \\
\text { in \% }\end{array}$ & $\begin{array}{r}2997383 \\
100,0\end{array}$ & - & - & - & - & : & - & $\begin{array}{r}125000 \\
4,2\end{array}$ & $\begin{array}{r}210383 \\
7,0\end{array}$ & - & - & $\begin{array}{r}2662000 \\
88,8\end{array}$ & - & . \\
\hline BMF & $\begin{array}{l}\text { in Schilling } \\
\text { in } \%\end{array}$ & - & & - & - & - & : & - & - & - & - & - & - & - & - \\
\hline BMLF & $\begin{array}{l}\text { in Schilling } \\
\text { in } \%\end{array}$ & $\begin{array}{r}41938202 \\
100,0\end{array}$ & $\begin{array}{r}656571 \\
1,6\end{array}$ & $\begin{array}{r}18907002 \\
45,0\end{array}$ & $\begin{array}{r}5389128 \\
12,9\end{array}$ & $\begin{array}{r}887844 \\
2,1\end{array}$ & - & - & - & $\begin{array}{r}1094033 \\
2,6\end{array}$ & $\begin{array}{r}4029804 \\
9,6\end{array}$ & $\begin{array}{r}245029 \\
0,6\end{array}$ & - & - & $\begin{array}{r}10728791 \\
25,6\end{array}$ \\
\hline BMWA & $\begin{array}{l}\text { in Schilling } \\
\text { in } \%\end{array}$ & $\begin{array}{r}763854649 \\
100,0\end{array}$ & $\begin{array}{r}1247600 \\
0,2\end{array}$ & $\begin{array}{r}14713801 \\
1,9\end{array}$ & $\begin{array}{r}516215030 \\
67,5\end{array}$ & $\begin{array}{r}59681475 \\
7,8\end{array}$ & $\begin{array}{r}32897189 \\
4,3\end{array}$ & - & $\begin{array}{r}117350376 \\
15,4\end{array}$ & $\begin{array}{r}2251700 \\
0,3\end{array}$ & $\begin{array}{r}16509970 \\
2,2\end{array}$ & $\begin{array}{r}195000 \\
0,0\end{array}$ & - & - & $\begin{array}{r}2792508 \\
0,4\end{array}$ \\
\hline \multirow{2}{*}{ INSGESAM } & in Schilling & 3371553313 & 200074677 & 67546696 & 808804887 & 115712133 & 108418121 & 31135690 & 504514946 & 240669075 & 193721251 & 20739016 & 2662000 & 6128343 & 1071426478 \\
\hline & in $\%$ & 100,0 & 5,9 & 2,0 & 24,0 & 3,4 & 3,2 & 0,9 & 15,0 & 7,1 & 5,7 & 0,6 & 0,1 & 0,2 & 31,9 \\
\hline
\end{tabular}

1) d.h. einschließlich Globalf̈̈rderungen für: Fonds zur Förderung der wissenschaftlichen Forschung, Forschungsförderungsfonds für die gewerbliche Wirtschaft, Ludwig Boltzmann-Gesellschaft, Österreichische Akademie der Wissenschaften, Österreichisches
Forschungszentrum Seibersdorf (insgesamt 2590670150 Schilling). Die in der Faktendokumentation 1999 nicht enthaltene Globalförderung seitens des BMWA an den FFF wurde unter Heranziehung des Bundesrechnungsabschlusses 1999 ermittelt. 
Tabelle 12: Forschungsförderungen und Forschungsaufträge des Bundes 1999 nach sozio-ökonomischen Zielsetzungen und vergebenden Ressorts

Auswertung der Faktendokumentation der Bundesdienststellen für 1999 (Stand: Dezember 2000) nach sozio-ökonomischen Zielsetzungen (ÖSTAT-Klassifikation), ohne "große" Globalförderungen ${ }^{1}$ )

\begin{tabular}{|c|c|c|c|c|c|c|c|c|c|c|c|c|c|c|c|}
\hline \multirow{2}{*}{\multicolumn{2}{|c|}{ RESSORTS }} & \multirow[b]{2}{*}{$\begin{array}{c}\text { Teilbeträge } \\
1999\end{array}$} & \multicolumn{13}{|c|}{ davon für } \\
\hline & & & \begin{tabular}{|c|} 
Förderung der \\
Erforschung \\
der Erde, \\
der Meere, \\
der Atmosphäre \\
und des \\
Weltraumes
\end{tabular} & $\begin{array}{c}\text { Förderung der } \\
\text { Land- und } \\
\text { Forstwirtschaft }\end{array}$ & $\begin{array}{c}\text { Förderung von } \\
\text { Handel, } \\
\text { Gewerbe und } \\
\text { Industrie }\end{array}$ & $\begin{array}{c}\text { Förderung der } \\
\text { Erzeugung, } \\
\text { Speicherung } \\
\text { und Verteilung } \\
\text { von Energie }\end{array}$ & $\begin{array}{l}\text { Förderung des } \\
\text { Transport-, } \\
\text { Verkehrs- und } \\
\text { Nachrichten- } \\
\text { wesens }\end{array}$ & $\begin{array}{l}\text { Förderung des } \\
\text { Unterrichts- } \\
\text { und Bildungs- } \\
\text { wesens }\end{array}$ & $\begin{array}{l}\text { Förderung des } \\
\text { Gesund- } \\
\text { heitswesens }\end{array}$ & $\begin{array}{c}\text { Förderung der } \\
\text { sozialen und } \\
\text { sozio-ökonomi- } \\
\text { schen Ent- } \\
\text { wicklung }\end{array}$ & $\begin{array}{l}\text { Förderung des } \\
\text { Umwelt- } \\
\text { schutzes }\end{array}$ & $\begin{array}{l}\text { Förderung der } \\
\text { Stadt-und } \\
\text { Raum- } \\
\text { planung }\end{array}$ & $\begin{array}{c}\text { Förderung der } \\
\text { Landesverteidi- } \\
\text { gung }\end{array}$ & $\begin{array}{l}\text { Förderung } \\
\text { anderer Ziel- } \\
\text { setzungen }\end{array}$ & $\begin{array}{l}\text { Förderung der } \\
\text { allgemeinen } \\
\text { Erweiterung } \\
\text { des Wissens }\end{array}$ \\
\hline BKA & $\begin{array}{l}\text { in Schilling } \\
\text { in \% }\end{array}$ & $\begin{array}{r}16817987 \\
100,0\end{array}$ & $\begin{array}{r}246656 \\
1,5\end{array}$ & $\begin{array}{r}1063313 \\
6,3\end{array}$ & $\begin{array}{r}86300 \\
0,5\end{array}$ & - & - & $\begin{array}{r}270000 \\
1,6\end{array}$ & $\begin{array}{r}3039975 \\
18,1\end{array}$ & $\begin{array}{r}2350012 \\
14,0\end{array}$ & $\begin{array}{r}7027935 \\
41,7\end{array}$ & - & - & - & $\begin{array}{r}2733796 \\
16,3\end{array}$ \\
\hline BMI & $\begin{array}{l}\text { in Schilling } \\
\text { in \% }\end{array}$ & $\begin{array}{r}75912 \\
100,0\end{array}$ & - & - & - & - & $\begin{array}{r}75912 \\
100,0\end{array}$ & - & - & - & - & - & - & - & - \\
\hline BMUKA & $\begin{array}{l}\text { in Schilling } \\
\text { in } \%\end{array}$ & $\begin{array}{r}14314114 \\
100,0\end{array}$ & - & - & - & - & - & $\begin{array}{r}3735550 \\
26,1\end{array}$ & - & $\begin{array}{r}1842140 \\
12,9\end{array}$ & - & - & - & - & $\begin{array}{r}8736424 \\
61,0\end{array}$ \\
\hline BMWV & $\begin{array}{l}\text { in Schilling } \\
\text { in } \%\end{array}$ & $\begin{array}{r}635628684 \\
100,0\end{array}$ & $\begin{array}{r}73058984 \\
11,5\end{array}$ & $\begin{array}{r}5956430 \\
0,9\end{array}$ & $\begin{array}{r}55853066 \\
8,8\end{array}$ & $\begin{array}{r}17746661 \\
2,8\end{array}$ & $\begin{array}{r}10481072 \\
1,6\end{array}$ & $\begin{array}{r}15277700 \\
2,4\end{array}$ & $\begin{array}{r}51517918 \\
8,1\end{array}$ & $\begin{array}{r}151575822 \\
23,8\end{array}$ & $\begin{array}{r}27957590 \\
4,4\end{array}$ & $\begin{array}{r}5951401 \\
0,9\end{array}$ & - & $\begin{array}{r}3548343 \\
0,6\end{array}$ & $\begin{array}{r}216703697 \\
34,2\end{array}$ \\
\hline BMAGS & $\begin{array}{l}\text { in Schilling } \\
\text { in } \%\end{array}$ & $\begin{array}{r}12359202 \\
100,0\end{array}$ & - & - & 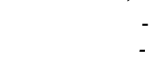 & - & - & - & $\begin{array}{r}713000 \\
5,8\end{array}$ & $\begin{array}{r}9010271 \\
72,9\end{array}$ & - & - & - & - & $\begin{array}{r}2635931 \\
21,3\end{array}$ \\
\hline BMUJF & $\begin{array}{l}\text { in Schilling } \\
\text { in } \%\end{array}$ & $\begin{array}{r}34188530 \\
100,0\end{array}$ & $\begin{array}{r}2146173 \\
6,3\end{array}$ & $\begin{array}{r}690000 \\
2,0\end{array}$ & $\begin{array}{r}478977 \\
1,4\end{array}$ & $\begin{array}{r}2081473 \\
6,1\end{array}$ & - & - & $\begin{array}{r}2829967 \\
8,3\end{array}$ & $\begin{array}{r}8913654 \\
26,1\end{array}$ & $\begin{array}{r}11518499 \\
33,6\end{array}$ & $\begin{array}{r}2289236 \\
6,7\end{array}$ & - & - & $\begin{array}{r}3240551 \\
9,5\end{array}$ \\
\hline BMAA & $\begin{array}{l}\text { in Schilling } \\
\text { in } \%\end{array}$ & - & - & - & - & - & - & - & - & - & - & - & - & - & - \\
\hline BMJ & $\begin{array}{l}\text { in Schilling } \\
\text { in } \%\end{array}$ & $\begin{array}{r}10000000 \\
100,0\end{array}$ & - & - & - & - & - & - & - & $\begin{array}{r}1000000 \\
100,0\end{array}$ & - & - & - & - & - \\
\hline BML & $\begin{array}{l}\text { in Schilling } \\
\text { in } \%\end{array}$ & $\begin{array}{r}2997383 \\
100,0\end{array}$ & - & - & . & - & - & - & $\begin{array}{r}125000 \\
4,2\end{array}$ & $\begin{array}{r}210383 \\
7,0\end{array}$ & - & - & $\begin{array}{r}26620000 \\
88,8\end{array}$ & - & - \\
\hline BMF & $\begin{array}{l}\text { in Schilling } \\
\text { in } \%\end{array}$ & & - & - & - & - & - & - & - & - & - & - & - & - & - \\
\hline BMLF & $\begin{array}{l}\text { in Schilling } \\
\text { in } \%\end{array}$ & $\begin{array}{r}41938202 \\
100,0\end{array}$ & $\begin{array}{r}656571 \\
1,6\end{array}$ & $\begin{array}{r}18907002 \\
45,0\end{array}$ & $\begin{array}{r}5389128 \\
12,9\end{array}$ & $\begin{array}{r}887844 \\
2,1\end{array}$ & - & - & - & $\begin{array}{r}1094033 \\
2,6\end{array}$ & $\begin{array}{r}4029804 \\
9,6\end{array}$ & $\begin{array}{r}245029 \\
0,6\end{array}$ & - & - & $\begin{array}{r}10728791 \\
25,6\end{array}$ \\
\hline BMWA & $\begin{array}{l}\text { in Schilling } \\
\text { in \% }\end{array}$ & $\begin{array}{r}21563149 \\
100,0\end{array}$ & $\begin{array}{r}1247600 \\
5,8\end{array}$ & $\begin{array}{r}392000 \\
1,8\end{array}$ & $\begin{array}{r}6022200 \\
28,0\end{array}$ & $\begin{array}{r}288000 \\
1,3\end{array}$ & $\begin{array}{r}3410000 \\
15,8\end{array}$ & - & $\begin{array}{r}730000 \\
3,4\end{array}$ & $\begin{array}{r}2251700 \\
10,4\end{array}$ & $\begin{array}{r}4234141 \\
19,6\end{array}$ & $\begin{array}{r}195000 \\
0,9\end{array}$ & - & - & $\begin{array}{r}2792508 \\
13,0\end{array}$ \\
\hline INSGESAMT & $\begin{array}{l}\text { in Schilling } \\
\text { in \% }\end{array}$ & $\begin{array}{r}780883163 \\
100,0\end{array}$ & $\begin{array}{r}77355984 \\
9,9\end{array}$ & $\begin{array}{r}27008745 \\
3,5\end{array}$ & $\begin{array}{r}67829671 \\
8,7\end{array}$ & $\begin{array}{r}21003978 \\
2,7\end{array}$ & $\begin{array}{r}13966984 \\
1,8\end{array}$ & $\begin{array}{r}19283250 \\
2,5\end{array}$ & $\begin{array}{r}58955860 \\
7,5\end{array}$ & $\begin{array}{r}178248015 \\
22,8\end{array}$ & $\begin{array}{r}54767969 \\
7,0\end{array}$ & $\begin{array}{r}8680666 \\
1,1\end{array}$ & $\begin{array}{r}2662000 \\
0,3\end{array}$ & $\begin{array}{r}3548343 \\
0,5\end{array}$ & $\begin{array}{r}247571698 \\
31,7\end{array}$ \\
\hline
\end{tabular}


Tabelle 13: Forschungsförderungen und Forschungsaufträge des Bundes 1999 nach Wissenschaftszweigen und vergebenden Ressorts

Auswertung der Faktendokumentation der Bundesdienststellen für 1999 (Stand: Dezember 2000) einschließlich "große" Globalförderungen ${ }^{1}$ )

\begin{tabular}{|c|c|c|c|c|c|c|c|c|}
\hline \multirow{2}{*}{\multicolumn{2}{|c|}{ RESSORTS }} & \multirow{3}{*}{$\begin{array}{r}\begin{array}{l}\text { Teilbeträge } \\
1999\end{array} \\
16817987 \\
100,0\end{array}$} & \multicolumn{6}{|c|}{ d a von für } \\
\hline & & & \multirow{2}{*}{$\begin{array}{r}\text { Naturwissen- } \\
\text { schaften } \\
8309476 \\
49,4\end{array}$} & \multirow{2}{*}{$\begin{array}{c}2.0 \\
\begin{array}{c}\text { Technische } \\
\text { Wissenschaften }\end{array} \\
86300 \\
0,5\end{array}$} & \multirow{2}{*}{$\begin{array}{r}3.0 \\
\text { Humanmedizin } \\
2051048 \\
12,2\end{array}$} & \multirow{2}{*}{$\begin{array}{c}4.0 \\
\begin{array}{c}\text { Land- und } \\
\text { Forstwirtschaft, } \\
\text { Veterinärmedizin }\end{array} \\
1488951 \\
8,9\end{array}$} & \multirow{2}{*}{\begin{aligned} \multicolumn{1}{c|}{5.0} \\
$\begin{array}{l}\text { Sozialwissen- } \\
\text { schaften }\end{array} \\
\begin{array}{r}882212 \\
29,0\end{array}\end{aligned}$} & \multirow{2}{*}{$\begin{array}{l}\quad 6.0 \\
\begin{array}{c}\text { Geisteswissen- } \\
\text { schaften }\end{array} \\
\end{array}$} \\
\hline BKA & $\begin{array}{l}\text { in Schilling } \\
\text { in \% }\end{array}$ & & & & & & & \\
\hline BMI & $\begin{array}{l}\text { in Schilling } \\
\text { in \% }\end{array}$ & $\begin{array}{r}75912 \\
100,0\end{array}$ & - & $\begin{array}{r}75912 \\
100,0\end{array}$ & - & - & - & - \\
\hline BMUKA & $\begin{array}{l}\text { in Schilling } \\
\text { in \% }\end{array}$ & $\begin{array}{r}14314114 \\
100,0\end{array}$ & $\begin{array}{r}160000 \\
1,1\end{array}$ & $\begin{array}{r}137670 \\
1,0\end{array}$ & - & - & $\begin{array}{r}7152619 \\
49,9\end{array}$ & $\begin{array}{r}6863825 \\
48,0\end{array}$ \\
\hline BMWV & $\begin{array}{l}\text { in Schilling } \\
\text { in \% }\end{array}$ & $\begin{array}{r}2484007334 \\
100,0\end{array}$ & $\begin{array}{r}963758003 \\
38,8\end{array}$ & $\begin{array}{r}487577841 \\
19,6\end{array}$ & $\begin{array}{r}366383511 \\
14,7\end{array}$ & $\begin{array}{r}41245365 \\
1,7\end{array}$ & $\begin{array}{r}321787541 \\
13,0\end{array}$ & $\begin{array}{r}303255073 \\
12,2\end{array}$ \\
\hline BMAGS & $\begin{array}{l}\text { in Schilling } \\
\text { in \% }\end{array}$ & $\begin{array}{r}12359202 \\
100,0\end{array}$ & - & $\begin{array}{l}- \\
-\end{array}$ & $\begin{array}{r}713000 \\
5,8\end{array}$ & - & $\begin{array}{r}11646202 \\
94,2\end{array}$ & $\begin{array}{l}- \\
-\end{array}$ \\
\hline BMUJF & $\begin{array}{l}\text { in Schilling } \\
\text { in } \%\end{array}$ & $\begin{array}{r}34188530 \\
100,0\end{array}$ & $\begin{array}{r}9613099 \\
28,1\end{array}$ & $\begin{array}{r}7787619 \\
22,8\end{array}$ & $\begin{array}{r}2770717 \\
8,1\end{array}$ & $\begin{array}{r}1899250 \\
5,6\end{array}$ & $\begin{array}{r}12067845 \\
35,3\end{array}$ & $\begin{array}{r}50000 \\
0,1\end{array}$ \\
\hline BMAA & $\begin{array}{l}\text { in Schilling } \\
\text { in \% }\end{array}$ & $\begin{array}{l}- \\
-\end{array}$ & - & $\begin{array}{l}- \\
-\end{array}$ & $\begin{array}{l}- \\
-\end{array}$ & - & - & $\begin{array}{l}- \\
-\end{array}$ \\
\hline BMJ & $\begin{array}{l}\text { in Schilling } \\
\text { in \% }\end{array}$ & $\begin{array}{r}1000000 \\
100,0\end{array}$ & - & - & - & - & $\begin{array}{r}1000000 \\
100,0\end{array}$ & - \\
\hline BML & $\begin{array}{l}\text { in Schilling } \\
\text { in \% }\end{array}$ & $\begin{array}{r}2997383 \\
100,0\end{array}$ & $\begin{array}{r}432000 \\
14,4\end{array}$ & $\begin{array}{r}2230000 \\
74,4\end{array}$ & - & - & $\begin{array}{r}335383 \\
11,2\end{array}$ & $\begin{array}{l}- \\
-\end{array}$ \\
\hline BMF & $\begin{array}{l}\text { in Schilling } \\
\text { in \% }\end{array}$ & $\begin{array}{l}- \\
-\end{array}$ & $\begin{array}{l}- \\
-\end{array}$ & $\begin{array}{l}- \\
-\end{array}$ & $\begin{array}{l}- \\
-\end{array}$ & - & - & - \\
\hline BMLF & $\begin{array}{l}\text { in Schilling } \\
\text { in \% }\end{array}$ & $\begin{array}{r}41938202 \\
100,0\end{array}$ & $\begin{array}{r}12728278 \\
30,4\end{array}$ & $\begin{array}{r}1261439 \\
3,0\end{array}$ & - & $\begin{array}{r}21260210 \\
50,6\end{array}$ & $\begin{array}{r}5519633 \\
13,2\end{array}$ & $\begin{array}{r}1168642 \\
2,8\end{array}$ \\
\hline BMWA & $\begin{array}{l}\text { in Schilling } \\
\text { in \% }\end{array}$ & $\begin{array}{r}763854649 \\
100,0\end{array}$ & $\begin{array}{r}58525396 \\
7,7\end{array}$ & $\begin{array}{r}625965785 \\
81,9\end{array}$ & $\begin{array}{r}44874383 \\
5,9\end{array}$ & $\begin{array}{r}30749582 \\
4,0\end{array}$ & $\begin{array}{r}3541101 \\
0,5\end{array}$ & $\begin{array}{r}198402 \\
0,0\end{array}$ \\
\hline INSGESA & $\begin{array}{l}\text { in Schilling } \\
\text { in \% }\end{array}$ & $\begin{array}{r}3371553313 \\
100,0\end{array}$ & $\begin{array}{r}1053526252 \\
31,2\end{array}$ & $\begin{array}{r}1125122566 \\
33,4\end{array}$ & $\begin{array}{r}416792659 \\
12,4\end{array}$ & $\begin{array}{r}96643358 \\
2,9\end{array}$ & $\begin{array}{r}367932536 \\
10,9\end{array}$ & $\begin{array}{r}311535942 \\
9,2\end{array}$ \\
\hline
\end{tabular}

1) d.h. einschließlich Globalförderungen für: Fonds zur Förderung der wissenschaftlichen Forschung, Forschungsförderungsfonds für die gewerbliche Wirtschaft, Ludwig Boltzmann-Gesellschaft, Österreichische Akademie der Wissenschaften, Österreichisches Forschungszentrum Seibersdorf (insgesamt 2590670150 Schilling). Die in der Faktendokumentation 1999 nicht enthaltene Globalförderung seitens des BMWA an den FFF wurde unter Heranziehung des

Bundesrechnungsabschlusses 1999 ermittelt. 
Tabelle 14: Forschungsförderungen und Forschungsaufträge des Bundes 1999 nach Wissenschaftszweigen und vergebenden Ressorts Auswertung der Faktendokumentation der Bundesdienststellen für 1999 (Stand: Dezember 2000) ohne "große" Globalförderungen ${ }^{1}$ )

\begin{tabular}{|c|c|c|c|c|c|c|c|c|}
\hline \multirow{2}{*}{\multicolumn{2}{|c|}{ RESSORTS }} & \multirow{3}{*}{$\begin{array}{l}\begin{array}{l}\text { Teilbeträge } \\
1999\end{array} \\
\begin{array}{r}16817987 \\
100,0\end{array}\end{array}$} & \multicolumn{6}{|c|}{ d a von für } \\
\hline & & & \multirow{2}{*}{$\begin{array}{r}\begin{array}{l}\text { Naturwissen- } \\
\text { schaften }\end{array} \\
8309476 \\
49,4\end{array}$} & \multirow{2}{*}{$\begin{array}{c}2.0 \\
\begin{array}{c}\text { Technische } \\
\text { Wissenschaften }\end{array} \\
86300 \\
0,5\end{array}$} & \multirow{2}{*}{$\begin{array}{r}3.0 \\
\text { Humanmedizin } \\
2051048 \\
12,2\end{array}$} & \multirow{2}{*}{$\begin{array}{c}4.0 \\
\begin{array}{c}\text { Land- und } \\
\text { Forstwirtschaft, } \\
\text { Veterinärmedizin }\end{array} \\
1488951 \\
8,9\end{array}$} & \multirow{2}{*}{\begin{aligned} \multicolumn{1}{c|}{5.0} \\
$\begin{array}{l}\text { Sozialwissen- } \\
\text { schaften }\end{array} \\
\begin{array}{r}882212 \\
29,0\end{array}\end{aligned}$} & \multirow{2}{*}{$\begin{array}{l}\quad 6.0 \\
\begin{array}{c}\text { Geisteswissen- } \\
\text { schaften }\end{array} \\
\end{array}$} \\
\hline BKA & $\begin{array}{l}\text { in Schilling } \\
\text { in } \%\end{array}$ & & & & & & & \\
\hline BMI & $\begin{array}{l}\text { in Schilling } \\
\text { in \% }\end{array}$ & $\begin{array}{r}75912 \\
100,0\end{array}$ & - & $\begin{array}{r}75912 \\
100,0\end{array}$ & - & - & - & - \\
\hline BMUKA & $\begin{array}{l}\text { in Schilling } \\
\text { in \% }\end{array}$ & $\begin{array}{r}14314114 \\
100,0\end{array}$ & $\begin{array}{r}160000 \\
1,1\end{array}$ & $\begin{array}{r}137670 \\
1,0\end{array}$ & - & - & $\begin{array}{r}7152619 \\
49,9\end{array}$ & $\begin{array}{r}6863825 \\
48,0\end{array}$ \\
\hline BMWV & $\begin{array}{l}\text { in Schilling } \\
\text { in \% }\end{array}$ & $\begin{array}{r}635628684 \\
100,0\end{array}$ & $\begin{array}{r}181775123 \\
28,6\end{array}$ & $\begin{array}{r}110472061 \\
17,4\end{array}$ & $\begin{array}{r}38091691 \\
6,0\end{array}$ & $\begin{array}{r}6810215 \\
1,1\end{array}$ & $\begin{array}{r}227954801 \\
35,8\end{array}$ & $\begin{array}{r}70524793 \\
11,1\end{array}$ \\
\hline BMAGS & $\begin{array}{l}\text { in Schilling } \\
\text { in \% }\end{array}$ & $\begin{array}{r}12359202 \\
100,0\end{array}$ & $\begin{array}{l}- \\
-\end{array}$ & $\begin{array}{l}- \\
-\end{array}$ & $\begin{array}{r}713000 \\
5,8\end{array}$ & - & $\begin{array}{r}11646202 \\
94,2\end{array}$ & - \\
\hline BMUJF & $\begin{array}{l}\text { in Schilling } \\
\text { in } \%\end{array}$ & $\begin{array}{r}34188530 \\
100,0\end{array}$ & $\begin{array}{r}9613099 \\
28,1\end{array}$ & $\begin{array}{r}7787619 \\
22,8\end{array}$ & $\begin{array}{r}2770717 \\
8,1\end{array}$ & $\begin{array}{r}1899250 \\
5,6\end{array}$ & $\begin{array}{r}12067845 \\
35,3\end{array}$ & $\begin{array}{r}50000 \\
0,1\end{array}$ \\
\hline BMAA & $\begin{array}{l}\text { in Schilling } \\
\text { in \% }\end{array}$ & - & $\begin{array}{l}- \\
-\end{array}$ & $\begin{array}{l}- \\
-\end{array}$ & - & - & - & $\begin{array}{l}- \\
-\end{array}$ \\
\hline BMJ & $\begin{array}{l}\text { in Schilling } \\
\text { in } \%\end{array}$ & $\begin{array}{r}1000000 \\
100,0\end{array}$ & $\begin{array}{l}- \\
-\end{array}$ & - & - & - & $\begin{array}{r}1000000 \\
100,0\end{array}$ & - \\
\hline BML & $\begin{array}{l}\text { in Schilling } \\
\text { in \% }\end{array}$ & $\begin{array}{r}2997383 \\
100,0\end{array}$ & $\begin{array}{r}432000 \\
14,4\end{array}$ & $\begin{array}{r}2230000 \\
74,4\end{array}$ & - & - & $\begin{array}{r}335383 \\
11,2\end{array}$ & - \\
\hline BMF & $\begin{array}{l}\text { in Schilling } \\
\text { in \% }\end{array}$ & - & $\begin{array}{l}- \\
-\end{array}$ & $\begin{array}{l}- \\
-\end{array}$ & - & - & - & - \\
\hline BMLF & $\begin{array}{l}\text { in Schilling } \\
\text { in } \%\end{array}$ & $\begin{array}{r}41938202 \\
100,0\end{array}$ & $\begin{array}{r}12728278 \\
30,4\end{array}$ & $\begin{array}{r}1261439 \\
3,0\end{array}$ & - & $\begin{array}{r}21260210 \\
50,6\end{array}$ & $\begin{array}{r}5519633 \\
13,2\end{array}$ & $\begin{array}{r}1168642 \\
2,8\end{array}$ \\
\hline BMWA & $\begin{array}{l}\text { in Schilling } \\
\text { in \% }\end{array}$ & $\begin{array}{r}21563149 \\
100,0\end{array}$ & $\begin{array}{r}4648146 \\
21,6\end{array}$ & $\begin{array}{r}11888500 \\
55,2\end{array}$ & $\begin{array}{r}545000 \\
2,5\end{array}$ & $\begin{array}{r}742000 \\
3,4\end{array}$ & $\begin{array}{r}3541101 \\
16,4\end{array}$ & $\begin{array}{r}198402 \\
0,9\end{array}$ \\
\hline INSGESA & $\begin{array}{l}\text { in Schilling } \\
\text { in \% }\end{array}$ & $\begin{array}{r}780883163 \\
100,0\end{array}$ & $\begin{array}{r}217666122 \\
27,9\end{array}$ & $\begin{array}{r}133939501 \\
17,2\end{array}$ & $\begin{array}{r}44171456 \\
5,7\end{array}$ & $\begin{array}{r}32200626 \\
4,1\end{array}$ & $\begin{array}{r}274099796 \\
35,0\end{array}$ & $\begin{array}{r}78805662 \\
10,1\end{array}$ \\
\hline
\end{tabular}

1) d.h. ohne Globalförderungen für: Fonds zur Förderung der wissenschaftlichen Forschung, Forschungsförderungsfonds für die gewerbliche Wirtschaft, Ludwig Boltzmann-Gesellschaft, Österreichische Akademie der Wissenschaften, Österreichisches Forschungszentrum Seibersdorf (insgesamt 2590670150 Schilling). Die in der Faktendokumentation 1999 nicht enthaltene Globalförderung seitens des BMWA an den FFF wurde unter Heranziehung des

Bundesrechnungsabschlusses 1999 ermittelt. 


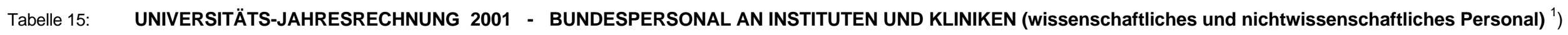

gegliedert nach Wissenschaftszweigen und Beschäftigtenkategorien

(VZÄ-Schätzung aufgrund des Stellenplanes 1. 1. 2001; 1 Planstelle = 1,0 VZÄ; auf der Basis der Koeffizienten aus der F\&E-Erhebung 1998)

Vollzeitäquivalente (VZÄ) für Lehre und Ausbildung, Forschung und experimentelle Entwicklung und Sonstige Tätigkeiten

\begin{tabular}{|c|c|c|c|c|c|c|c|c|c|c|c|c|c|c|c|c|c|c|c|c|c|c|c|c|}
\hline \multirow{3}{*}{ WISSENSCHAFTSZWEIGE } & \multicolumn{4}{|c|}{ Professoren } & \multicolumn{4}{|c|}{$\begin{array}{l}\text { Assistenten, } \\
\text { Universitätsdozenten }\end{array}$} & \multicolumn{4}{|c|}{$\begin{array}{c}\text { Sonstiges wissenschaftliches } \\
\text { Personal }\end{array}$} & \multicolumn{4}{|c|}{$\begin{array}{c}\text { Wissenschaftliches Personal } \\
\text { zusammen }\end{array}$} & \multicolumn{4}{|c|}{$\begin{array}{c}\text { Nichtwissenschaftliches } \\
\text { Personal }\end{array}$} & \multicolumn{4}{|c|}{ INSGESAMT } \\
\hline & 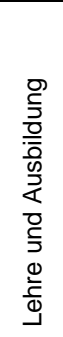 & 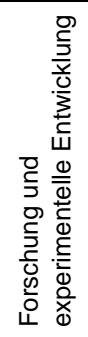 & 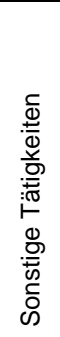 & $\begin{array}{l}\bar{\complement} \\
\stackrel{\Phi}{\xi} \\
\stackrel{్}{\Phi} \\
\stackrel{N}{N} \\
N\end{array}$ & 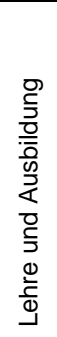 & 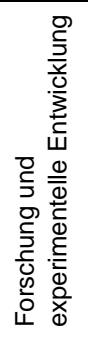 & 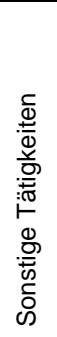 & 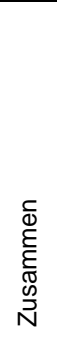 & 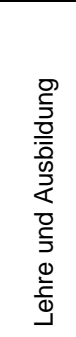 & 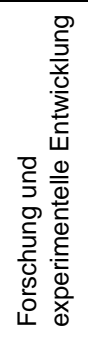 & 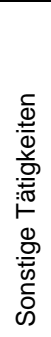 & 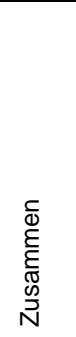 & 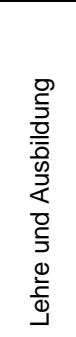 & 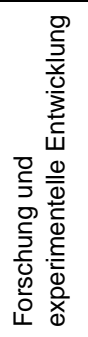 & 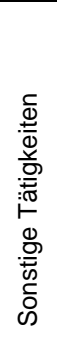 & $\begin{array}{l}\bar{\complement} \\
\stackrel{\Phi}{\xi} \\
\stackrel{్}{\Phi} \\
\stackrel{\omega}{N} \\
N\end{array}$ & 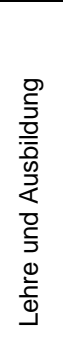 & 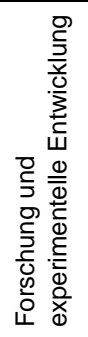 & 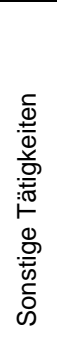 & 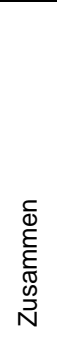 & 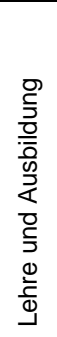 & 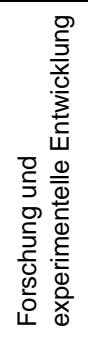 & 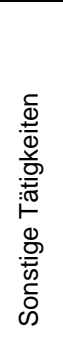 & 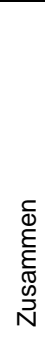 \\
\hline & & & & & & & & & & & Vo & it & iv & it & & & & & & & & & & \\
\hline
\end{tabular}

1.0 Naturwissenschaften

2.0 Technische Wissenschaften

3.0 Humanmedizin ohne Kliniken Kliniken

einschließlich Klinike 4.0 Land- und Forstwirtschaft, Veterinärmedizin

\section{0 bis 4.0 Zwischensumme}

ohne Kliniken einschließlich Kliniken

5.0 Sozialwissenschaften

6.0 Geisteswissenschaften

5.0 und 6.0 Zwischensumme

1.0 bis $6.0 \quad$ INSGESAMT

ohne Kliniken

einschließlich Kliniken

Stand: April 2001

\section{$203,9 \quad 227,8$}

$103,1 \quad 103,2$

$48,3 \quad 64,6$

$45,4 \quad 61,4$

$93,7 \quad 126,0$

$25,0 \quad 23,3$

$380,3 \quad 418$

$425,7 \quad 480,3$

$223,0 \quad 223,7$

$167,7 \quad 160,7$

$390,7 \quad 384,4$

$771,0 \quad 803,3$

$816,4 \quad 864,7$

\section{$\begin{array}{llllll}45,3 & 477,0 & 627,6 & 797,8 & 112,4 & 1.537,8\end{array}$}

$\begin{array}{llllll}18,7 & 225,0 & 382,0 & 404,7 & 59,6 & 846,3\end{array}$

$\begin{array}{llllll}15,1 & 128,0 & 218,9 & 315,9 & 58,6 & 593,4\end{array}$

$\begin{array}{lllllll}84,2 & 191,0 & 409,7 & 672,2 & 1.381,1 & 2.463,0\end{array}$

$\begin{array}{llllll}99,3 & 319,0 & 628,6 & 988,1 & 1.439,7 & 3.056,4\end{array}$

$\begin{array}{llllll}8,7 & 57,0 & 75,6 & 97,8 & 45,6 & 219,0\end{array}$

\section{1,6}

$31,0 \quad 49,3$

$7,2 \quad 20,1$

$5,9 \quad 15,7$

$13,1 \quad 35,8$

$5,7 \quad 13,3$
$87,8 \quad 887,0 \quad 1.304,1 \quad 1.616,2 \quad 276,2 \quad 3.196,5$

$95,5 \quad 174,7$ $101,4 \quad 190,4$

$58,7 \quad 40,9$

$223,0 \quad 77,5$

$\begin{array}{llllll}34,3 & 481,0 & 550,7 & 614,1 & 96,2 & 1,261,0\end{array}$

$27,6 \quad 356,0 \quad 306,3 \quad 326,7$

$63,0 \quad 696,0$

$\begin{array}{llllll}61,9 & 837,0 & 857,0 & 940,8 & 159,2 & 1.957,0\end{array}$

$\begin{array}{llllll}149,7 & 1.724,0 & 2.161,1 & 2.557,0 & 435,4 & 5.153,5\end{array}$

$\begin{array}{lllllll}233,9 & 1.915,0 & 2.570,8 & 3.229,2 & 1.816,5 & 7.616,5\end{array}$
$377,2 \quad 293,1$ $383,1 \quad 308,8$ $\begin{array}{llllll}52,4 & 322,6 & 1.779,9 & 2.209,8 & 416,4 & 4.406,1\end{array}$

$\begin{array}{lllllll}78,3 & 370,1 & 2.240,9 & 2.959,1 & 1.907,6 & 7.107,6\end{array}$

$\begin{array}{lllllll}11,3 & 110,9 & 832,4 & 878,7 & 141,8 & 1.852,9\end{array}$

$\begin{array}{llllll}21,0 & 321,5 & 697,0 & 564,9 & 111,6 & 1.373,5\end{array}$

$\begin{array}{llllll}32,3 & 432,4 & 1.529,4 & 1.443,6 & 253,4 & 3.226,4\end{array}$ 110,6
$24,1 \quad 167,7$

$\begin{array}{r}7,3 \\ 25,9 \\ \hline 33\end{array}$

33,2

3,7 $\begin{array}{rrrrr}97,6 & 516,1 & 557,2 & 95,6 & 1.168,9\end{array}$

$400,6 \quad 81,0 \quad 756,0$

$82,1 \quad 735,4 \quad 1.149,9 \quad 1.572,2 \quad 3.457,5$

$\begin{array}{lll}3,7 & 22,7 & 106,3 \\ & 134,4\end{array}$

298,7 $\begin{array}{llllllll}362,5 & 699,3 & 184,7 & 1.246,5 & 1.245,6 & 1.816,9 & 366,5 & 3.429,0\end{array}$

$\begin{array}{llllllll}221,7 & 357,7 & 111,1 & 690,5 & 737,8 & 914,9 & 206,7 & 1.859,4\end{array}$

$\begin{array}{llllllll}184,1 & 420,9 & 132,0 & 737,0 & 458,5 & 821,5 & 213,0 & 1.493,0\end{array}$

$\begin{array}{lllllllll}89,0 & 315,4 & 414,1 & 818,5 & 550,0 & 1.064,7 & 1905,3 & 3520,0\end{array}$

$\begin{array}{llllllll}273,1 & 736,3 & 546,1 & 1.555,5 & 1.008,5 & 1.886,2 & 2.118,3 & 5.013,0\end{array}$

$\begin{array}{llllllll}10,8 & 205,7 & 102,0 & 418,5 & 217,1 & 340,1 & 160,0 & 717,2\end{array}$

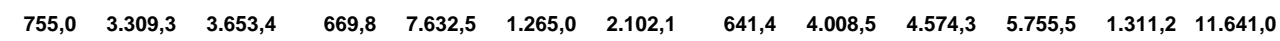

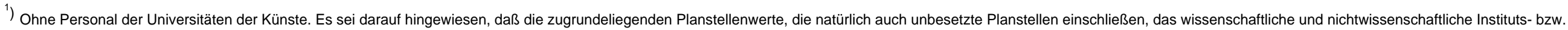

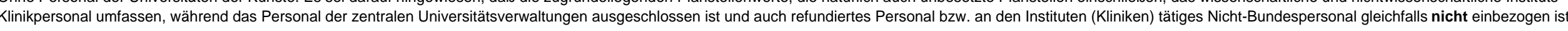




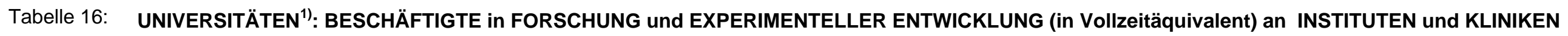
im Jahre 1998

gegliedert nach Wissenschaftszweigen und Beschäftigtenkategorien

\begin{tabular}{|c|c|}
\hline Wissenschaftszweige & \\
& \\
& \\
& \\
& \\
\hline
\end{tabular}

1.0 Naturwissenschaften

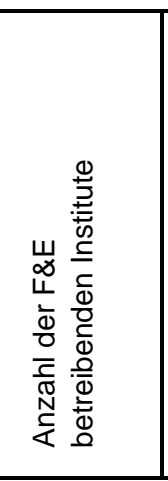

2.0 Technische Wissenschaften

3.0 Humanmedizin ohne Kliniken Kliniken einschließlich Kliniken

\section{1}
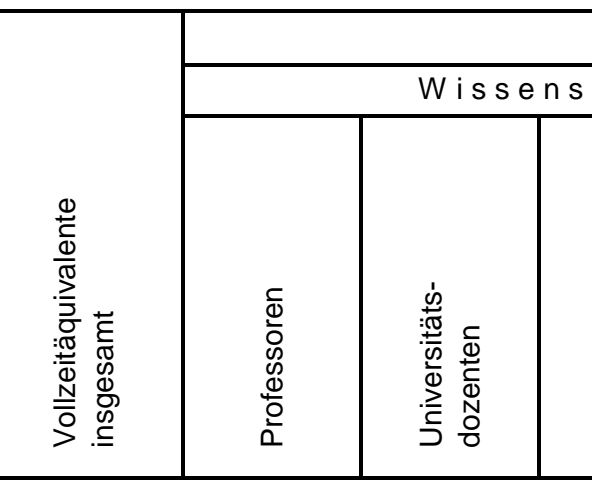

VOLLZEITÄQUIVALENTE für F\&E

L.0 Land- u. Forstwirtschaft,
Veterinärmedizin

4095,5

218,2

287,1

4095,5

102,4

287,1

1736,4

63,3

$48,3 \quad 345,4$

4118,5

67,8

84,9

131,7

5854,9

131,0

216,6

$\begin{array}{lll}47 & \mathbf{8 1 1}, 0 & 28,3\end{array}$

14,5

90,7

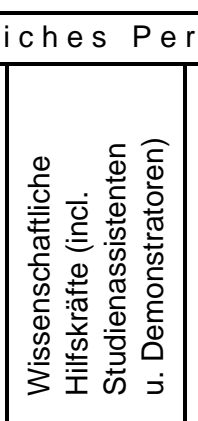

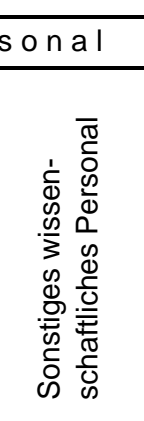

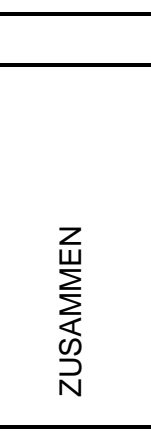

\begin{tabular}{|c|}
\hline 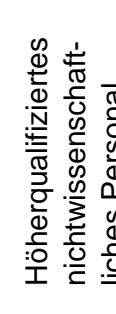 \\
\hline
\end{tabular}

827,2

1811,5

308,5

$310,8 \quad 810,6$

142,8

\section{4,5}

488,6

488,6
693,2

$5,7 \quad 177,3 \quad 535,6$

$\begin{array}{lrr}5,7 & 171,9 & 822,0\end{array}$

$\begin{array}{rr}131,9 & 822,0 \\ 309,2 & 1357,6\end{array}$

\section{9,2}

530,8

0,2

$82,5 \quad 216,3$

92,2

118,4

\section{0 bis $4.0 \quad$ Zwischensumme ohne Kliniken einschließlich Kliniken}

5.0 Sozialwissenschaften

6.0 Geisteswissenschaften

5.0 und $6.0 \quad$ Zwischensumme

1.0 bis $6.0 \quad$ INSGESAMT ohne Kliniken einschließlich Kliniken

\section{$478 \quad 8807,2$}

$412,2 \quad 434,8 \quad 1108,2$

$\begin{array}{lll}412,2 & 434,8 & 1108,2 \\ 480,0 & 566,5 & 1596,8\end{array}$

21,0
23,0

$1397,8 \quad 3374,1$

802,7

1074,3

969,3

1153,5

5146,1

$$
12925,8
$$

216,8

88,5

429,7

1645,8

149,6

86,7

212,0

9,4

1529,7

4196,0

71,8

162,9

344

3848,2

366,4

175,1

641,7

12,1

138,0

588,9

44,5

110,7

744,0

\section{5,4}

778,6

$609,9 \quad 1749,9$

$\begin{array}{ll}741,7 & 2238,6\end{array}$

35,1

1761,8

5623,5

919,0

1190,6

1242,8

1242,8
1427,0

6963,3

Quelle: Statistik Austria (Bundesanstalt Statistik Österreich)

Stand: April 2001

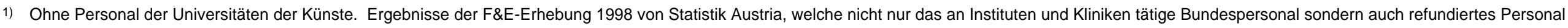
und sonstiges "Drittmittelpersonal" erfaßte. -- Rundungsdifferenzen. 
UNIVERSITÄTEN ${ }^{1)}$ - BESCHÄFTIGTE AN INSTITUTEN UND KLINIKEN (wissenschaftliches und nichtwissenschaftliches Personal) im Jahre 1998 gegliedert nach Wissenschaftszweigen und Beschäftigtenkategorien

Arbeitszeitverteilung mit aufgeteiltem Verwaltungsanteil in Prozent

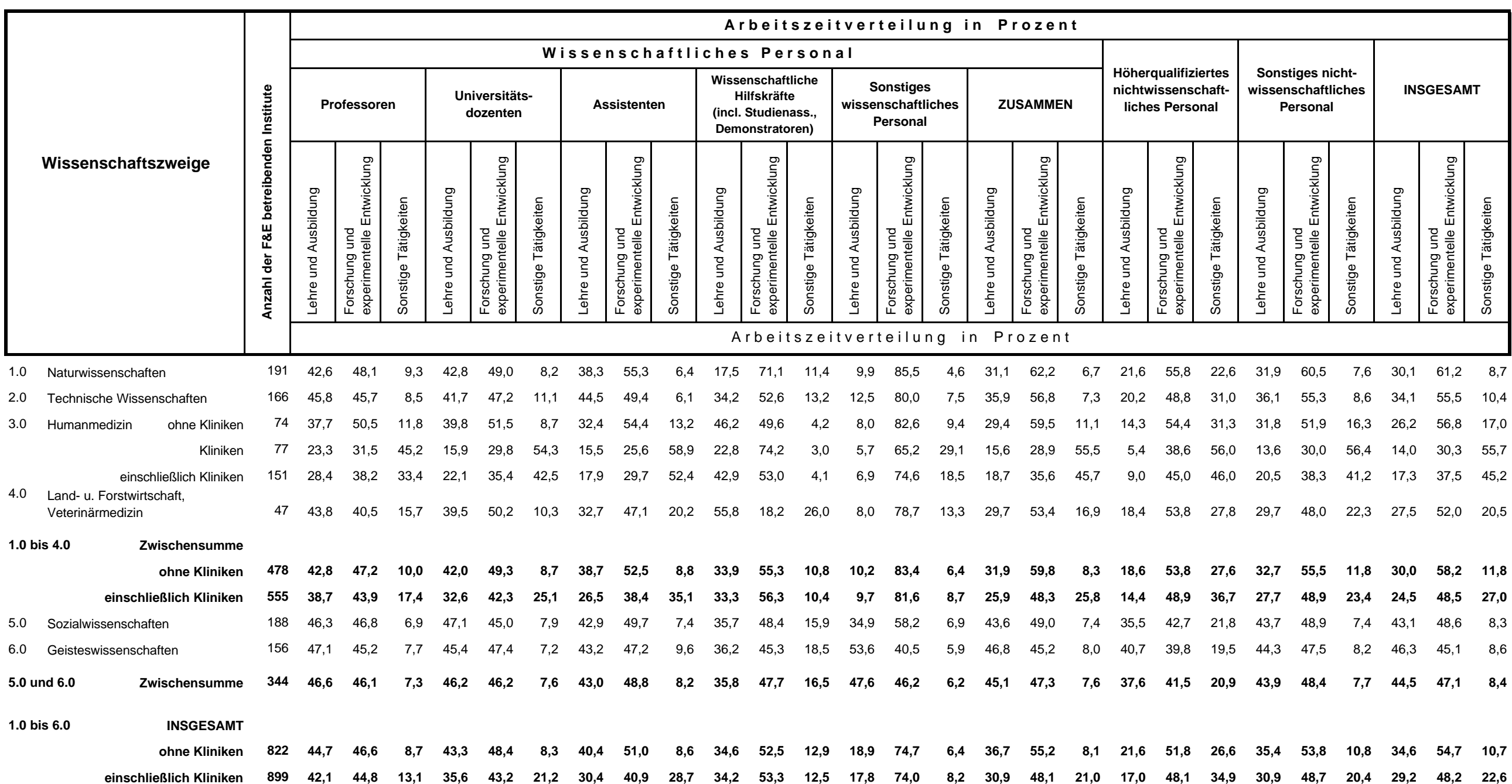

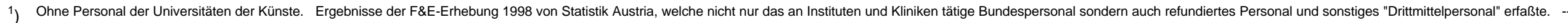
Rundungsdifferenzen. 
Tabelle 18: UNIVERSITÄTEN ${ }^{1)}$ - WISSENSCHAFTLICHES PERSONAL (in Kopfzahlen und in Vollzeitäquivalenten für F\&E) an INSTITUTEN und KLINIKEN im Jahre 1998

gegliedert nach Wissenschaftszweigen, Beschäftigtenkategorien, Altersgruppen und Geschlecht

\subsection{NATURWISSENSCHAFTEN}

\begin{tabular}{|c|c|c|c|c|c|c|c|c|c|c|c|c|}
\hline \multirow{4}{*}{ Beschäftigten-Kategorien } & \multicolumn{12}{|c|}{ Wissenschaftliches Personal insgesamt in } \\
\hline & \multirow{3}{*}{$\begin{array}{l}\text { Kopf- } \\
\text { zahlen }\end{array}$} & \multicolumn{11}{|c|}{ Vollzeitäquivalenten (VZÄ) für F\&E } \\
\hline & & \multirow{2}{*}{$\begin{array}{l}\text { INSGE- } \\
\text { SAMT }\end{array}$} & \multicolumn{10}{|c|}{ davon VZÄ für Beschäftigte im Alter von: } \\
\hline & & & $\begin{array}{l}\text { unter } 25 \\
\text { Jahren }\end{array}$ & $\begin{array}{c}25 \text { bis } 29 \\
\text { Jahre }\end{array}$ & $\begin{array}{c}30 \text { bis } 34 \\
\text { Jahre }\end{array}$ & $\begin{array}{c}35 \text { bis } 39 \\
\text { Jahre }\end{array}$ & $\begin{array}{c}40 \text { bis } 44 \\
\text { Jahre }\end{array}$ & $\begin{array}{l}45 \text { bis } 49 \\
\text { Jahre }\end{array}$ & $\begin{array}{c}50 \text { bis } 54 \\
\text { Jahre }\end{array}$ & $\begin{array}{c}55 \text { bis } 59 \\
\text { Jahre }\end{array}$ & $\begin{array}{c}60 \text { bis } 64 \\
\text { Jahre }\end{array}$ & $\begin{array}{l}65 \mathrm{~J} . \mathrm{u} . \\
\text { darüber }\end{array}$ \\
\hline \multicolumn{13}{|l|}{ Professoren } \\
\hline männlich & 475 & 209,1 & - & 0,7 & 0,8 & 4,8 & 13,0 & 24,8 & 40,3 & 71,3 & 27,9 & 25,6 \\
\hline weiblich & 15 & 8,6 & - & - & - & 0,6 & - & 1,1 & 1,7 & 2,1 & 1,1 & 2,1 \\
\hline Zusammen & 490 & 217,7 & - & 0,7 & 0,8 & 5,4 & 13,0 & 25,9 & 42,0 & 73,4 & 29,0 & 27,7 \\
\hline \multicolumn{13}{|l|}{ Universitätsdozenten } \\
\hline männlich & 556 & 266,1 & - & 0,2 & 7,8 & 36,6 & 56,8 & 52,9 & 66,6 & 39,8 & 5,1 & 0,4 \\
\hline weiblich & 43 & 21,0 & - & - & 1,3 & 4,4 & 4,4 & 4,6 & 3,1 & 3,2 & - & - \\
\hline Zusammen & 599 & 287,1 & - & 0,2 & 9,1 & 41,1 & 61,1 & 57,5 & 69,7 & 43,0 & 5,1 & 0,4 \\
\hline \multicolumn{13}{|l|}{ Assistenten } \\
\hline männlich & 931 & 387,8 & 2,0 & 73,5 & 131,7 & 75,4 & 48,0 & 24,7 & 21,2 & 8,7 & 2,3 & 0,4 \\
\hline weiblich & 229 & 79,8 & 0,4 & 20,1 & 26,9 & 14,7 & 9,8 & 3,7 & 2,7 & 0,8 & 0,9 & - \\
\hline Zusammen & 1160 & 467,7 & 2,3 & 93,6 & 158,6 & 90,1 & 57,7 & 28,4 & 23,9 & 9,5 & 3,3 & 0,4 \\
\hline \multicolumn{13}{|l|}{ Wissenschaftliche Hilfskräfte ${ }^{<}$) } \\
\hline männlich & 35 & 10,6 & 1,2 & 2,9 & 4,6 & 1,4 & - & 0,1 & 0,4 & - & - & - \\
\hline weiblich & 8 & 0,8 & 0,6 & 0,2 & - & - & - & - & - & - & - & - \\
\hline Zusammen & 43 & 11,4 & 1,8 & 3,1 & 4,6 & 1,4 & - & 0,1 & 0,4 & - & - & - \\
\hline \multicolumn{13}{|l|}{ Sonst. wissensch. Personal } \\
\hline männlich & 1114 & 625,8 & 17,4 & 268,0 & 194,8 & 76,0 & 25,0 & 13,1 & 13,9 & 15,7 & 2,0 & - \\
\hline weiblich & 398 & 199,2 & 9,4 & 101,3 & 54,6 & 12,8 & 8,5 & 6,6 & 2,4 & 3,7 & - & - \\
\hline Zusammen & 1512 & 825,0 & 26,7 & 369,3 & 249,3 & 88,8 & 33,5 & 19,8 & 16,3 & 19,4 & 2,0 & - \\
\hline \multicolumn{13}{|l|}{ SUMME wissensch. Personal } \\
\hline männlich & 3111 & 1499,4 & 20,5 & 345,4 & 339,6 & 194,1 & 142,7 & 115,6 & 142,5 & 135,5 & 37,3 & 26,4 \\
\hline weiblich & 693 & 309,4 & 10,3 & 121,6 & 82,8 & 32,6 & 22,6 & 16,0 & 9,8 & 9,7 & 2,0 & 2,1 \\
\hline Zusammen & 3804 & 1808,8 & 30,8 & 467,0 & 422,3 & 226,7 & 165,3 & 131,5 & 152,3 & 145,2 & 39,3 & 28,5 \\
\hline
\end{tabular}

Stand: April 2001

Quelle: Statistik Austria (Bundesanstalt Statistik Osterreich)

1) Ohne Personal der Universitäten der Künste. Ergebnisse der F\&E-Erhebung 1998 von Statistik Austria, welche nicht nur das an Instituten und Kliniken tätige Bundespersonal sondern auch refundiertes Personal und sonstiges "Drittmittelpersonal" erfaßte. -- ${ }^{2}$ ) Diese Personalkategorie schließt Studienassistenten und Demonstratoren ein. --- Rundungsdifferenzen. 
Tabelle 18 (Fortsetzung 1)

2.0 TECHNISCHE WISSENSCHAFTEN

\begin{tabular}{|c|c|c|c|c|c|c|c|c|c|c|c|c|}
\hline \multirow{4}{*}{ Beschäftigten-Kategorien } & \multicolumn{12}{|c|}{ Wissenschaftliches Pe } \\
\hline & \multirow{3}{*}{$\begin{array}{l}\text { Kopf- } \\
\text { zahlen }\end{array}$} & \multicolumn{11}{|c|}{ Vollzeitäquivalenten (VZÄ) für F\&E } \\
\hline & & \multirow{2}{*}{$\begin{array}{l}\text { INSGE- } \\
\text { SAMT }\end{array}$} & \multicolumn{10}{|c|}{ davon VZÄ für Beschäftigte im Alter von: } \\
\hline & & & $\begin{array}{c}\text { unter } 25 \\
\text { Jahren }\end{array}$ & $\begin{array}{c}25 \text { bis } 29 \\
\text { Jahre }\end{array}$ & $\begin{array}{c}30 \text { bis } 34 \\
\text { Jahre }\end{array}$ & $\begin{array}{c}35 \text { bis } 39 \\
\text { Jahre }\end{array}$ & $\begin{array}{c}40 \text { bis } 44 \\
\text { Jahre }\end{array}$ & $\begin{array}{c}45 \text { bis } 49 \\
\text { Jahre }\end{array}$ & $\begin{array}{c}50 \text { bis } 54 \\
\text { Jahre }\end{array}$ & $\begin{array}{c}55 \text { bis } 59 \\
\text { Jahre }\end{array}$ & $\begin{array}{c}60 \text { bis } 64 \\
\text { Jahre }\end{array}$ & $\begin{array}{l}65 \mathrm{~J} . \mathrm{u} . \\
\text { darüber }\end{array}$ \\
\hline
\end{tabular}

Professoren

männlich

weiblich

Zusammen

$238 \quad 100,9$

$4 \quad 1,4$

$242 \quad 102,2$

Universitätsdozenten

männlich

weiblich

Zusammen

$101 \quad 48,2$

20,2

Assistenten

männlich

weiblich

Zusammen

$784 \quad 312,9$

$103 \quad 32,4$

345,4

0,0

0,5
-
0,5

0,5
-
0,5

3,9
0,5
4,5

7,2
-
7,2

$\begin{array}{rr}6,4 & 12,3 \\ 0,1 & 0,2 \\ 6,5 & 12,5\end{array}$

35,6

$\begin{array}{rr}18,2 & 16,4 \\ - & -\end{array}$

haftliche Hilfskräfte

männlich
weiblich

Zusammen

$21 \quad 3,3$

$4 \quad 0,5$

25

0,5
3,7

$\begin{array}{ll}0,4 & 2,3 \\ 0,4 & 0,0 \\ 0,8 & 2,3\end{array}$

$530 \quad 284,5$

männlich

weiblich

Zusammen

$49 \quad 26,0$

$579 \quad 310,5$

$\begin{array}{rrrr}3,5 & 119,1 & 88,5 & 32,2 \\ 1,2 & 13,2 & 6,7 & 2,0 \\ 4,7 & 132,4 & 95,2 & 34,2\end{array}$

6,4
-
6,4

14,4
-
14,4

7,9

7,6

36,1

18,2

16,4

wissensch. Personal

männlich
weiblich

Zusammen

$\begin{array}{rr}1674 & 749 \\ 162 & 60 \\ 1836 & 810,2\end{array}$

$\begin{array}{rrr}749,7 & 3,9 & 206,8 \\ 60,4 & 1,6 & 23,3 \\ 810,2 & 5,5 & 230\end{array}$

$\begin{array}{rr}206,8 & 219,2 \\ 23,3 & 19,2 \\ 230,2 & 238,3\end{array}$

219,2
19,2
238,3

$\begin{array}{rrr}89,0 & 53,3 & 33,7 \\ 8,2 & 2,7 & 1,1 \\ 97,1 & 56,0 & 34,9\end{array}$

35,8
1,6
37,4

60,2
2,2

31,0
0,6

31,6

16,9

16,9 
Tabelle 18 (Fortsetzung 2)

\subsection{HUMANMEDIZIN}

\begin{tabular}{|c|c|c|c|c|c|c|c|c|c|c|c|c|}
\hline \multirow{4}{*}{ Beschäftigten-Kategorien } & \multicolumn{12}{|c|}{ Wissenschaftliches Personal insgesamt in } \\
\hline & \multirow{3}{*}{$\begin{array}{l}\text { Kopf- } \\
\text { zahlen }\end{array}$} & \multicolumn{11}{|c|}{ Vollzeitäquivalenten (VZÄ) für F\&E } \\
\hline & & \multirow{2}{*}{$\begin{array}{l}\text { INSGE- } \\
\text { SAMT }\end{array}$} & \multicolumn{10}{|c|}{ davon VZÄ für Beschäftigte im Alter von: } \\
\hline & & & $\begin{array}{c}\text { unter } 25 \\
\text { Jahren }\end{array}$ & $\begin{array}{c}25 \text { bis } 29 \\
\text { Jahre }\end{array}$ & $\begin{array}{c}30 \text { bis } 34 \\
\text { Jahre }\end{array}$ & $\begin{array}{c}35 \text { bis } 39 \\
\text { Jahre }\end{array}$ & $\begin{array}{c}40 \text { bis } 44 \\
\text { Jahre }\end{array}$ & $\begin{array}{c}45 \text { bis } 49 \\
\text { Jahre }\end{array}$ & $\begin{array}{c}50 \text { bis } 54 \\
\text { Jahre }\end{array}$ & $\begin{array}{c}55 \text { bis } 59 \\
\text { Jahre }\end{array}$ & $\begin{array}{c}60 \text { bis } 64 \\
\text { Jahre }\end{array}$ & $\begin{array}{l}65 \mathrm{~J} . \mathrm{u} . \\
\text { darüber }\end{array}$ \\
\hline
\end{tabular}

Professoren

männlich

weiblich

Zusammen

$326 \quad 120,6$

$27 \quad 10,5$

Universitätsdozenten

männlich

weiblich

Zusammen

$533 \quad 188,8$

$\begin{array}{rr}533 & 188,8 \\ 91 & 27,7\end{array}$

$624 \quad 216,5$

Assistenten

männlich

weiblich

Zusammen

$1774 \quad 462,9$

$949 \quad 230,3$

$2723 \quad 693,2$

0,2

0,2

35,4
30,6

30,6

$65,9-72,9$

$\begin{array}{rr}- & 3,1 \\ 0,8 & 1,0\end{array}$

$3,1 \quad 14,3$

14,3

17,6
2,2
19,8

26,8

$26,8 \quad 35,4$

$35,4 \quad 12,4$

Wissenschaftliche Hilfskräfte

$$
\begin{aligned}
& \text { männlich } \\
& \text { weiblich } \\
& \text { Zusammen }
\end{aligned}
$$

$45 \quad 2,6$

83

2,6
5,0
7,6

$0,2 \quad 1,4$

$1,4 \quad 0,1$

Sonst. wissensch. Personal

$$
\begin{aligned}
& \text { männlich } \\
& \text { weiblich }
\end{aligned}
$$

Zusammen

$280 \quad 155,5$

$\begin{array}{ll}280 & 155,5 \\ 277 & 153,6\end{array}$

$557 \quad 309,1$

0,7
0,9

4,7

0,1
0,6
0,7

2,6

16,5

19,8

27,2

37,5

1,0
13,5

11,0

wissensch. Personal

männlich

weiblich

Zusammen

2958
1382

$\begin{array}{lr}1382 & 427,1 \\ 4340 & 1357,4\end{array}$

$4,4 \quad 38,6$

$38,6 \quad 52,8$

44,3
97,1

42,8
6,5
49,3

56,6

$36,5 \quad 25,5$

25,5
3,8

3,8
29,3

17,2

1,4

6,3

6,3

1,4

$\begin{array}{lll}6,6 & 67,3 & 41,7\end{array}$

18,6

3,1

$\begin{array}{rr}144,3 & 64,3 \\ 71,2 & 28,0\end{array}$

$215,4 \quad 28,0$

32,2

8,8

16,0
9,7

25,7

12,5
8,0

20,5

1,1

$\begin{array}{rr}- & 1,0 \\ 0,4 & - \\ 0,4 & 1,0\end{array}$

11,7

100,7

$\begin{array}{rr}32,7 & 11,5 \\ 13,0 & 8,7 \\ 45,7 & 20,2\end{array}$

3,7
9,2
12,9

5,1
4,3

4,3
9,4

$\begin{array}{ll}5,2 & 1,6 \\ 2,2 & 2,3 \\ 7,3 & 3,9\end{array}$

$\begin{array}{rrrrrr}4,8 & 75,3 & 210,4 & 222,9 & 147,6 & 90,0 \\ 8,2 & 96,0 & 118,6 & 92,1 & 49,7 & 25,3 \\ 13,0 & 171,3 & 329,0 & 314,9 & 197,3 & 115,3\end{array}$

90,0
25,3
115,3

73,3

73,3
18,3
91,7

70,2
13,6
83,9

23,4
4,5

4,5
27,9

12,4

0,7
13,1 
Tabelle 18 (Fortsetzung 3)

4.0 LAND- Und FORSTWIRTSCHAFT, VETERINÄRMEDIZIN

\begin{tabular}{|c|c|c|c|c|c|c|c|c|c|c|c|c|}
\hline \multirow{4}{*}{ Beschäftigten-Kategorien } & \multicolumn{12}{|c|}{ Wissenschaftliches Personal insgesamt in } \\
\hline & \multirow{3}{*}{$\begin{array}{l}\text { Kopf- } \\
\text { zahlen }\end{array}$} & \multicolumn{11}{|c|}{ Vollzeitäquivalenten (VZÄ) für F\&E } \\
\hline & & \multirow{2}{*}{$\begin{array}{l}\text { INSGE- } \\
\text { SAMT }\end{array}$} & \multicolumn{10}{|c|}{ davon VZÄ für Beschäftigte im Alter von: } \\
\hline & & & $\begin{array}{c}\text { unter } 25 \\
\text { Jahren }\end{array}$ & $\begin{array}{c}25 \text { bis } 29 \\
\text { Jahre }\end{array}$ & $\begin{array}{c}30 \text { bis } 34 \\
\text { Jahre }\end{array}$ & $\begin{array}{c}35 \text { bis } 39 \\
\text { Jahre }\end{array}$ & $\begin{array}{c}40 \text { bis } 44 \\
\text { Jahre }\end{array}$ & $\begin{array}{c}45 \text { bis } 49 \\
\text { Jahre }\end{array}$ & $\begin{array}{c}50 \text { bis } 54 \\
\text { Jahre }\end{array}$ & $\begin{array}{c}55 \text { bis } 59 \\
\text { Jahre }\end{array}$ & $\begin{array}{c}60 \text { bis } 64 \\
\text { Jahre }\end{array}$ & $\begin{array}{l}65 \mathrm{~J} . \mathrm{u} . \\
\text { darüber }\end{array}$ \\
\hline
\end{tabular}

Professoren

männlich

weiblich

Zusammen

$68 \quad 26,0$

$7 \quad 2,3$

Universitätsdozenten

männlich

weiblich

Zusammen

$75 \quad 28,3$

2,3
28,3

$30 \quad 14,0$

$1 \quad 0,6$

Assistenten

männlich

weiblich

Zusammen

$131 \quad 60,6$
82

30,1

Wissenschaftliche Hilfskräfte

$$
\begin{aligned}
& \text { männlich } \\
& \text { weiblich }
\end{aligned}
$$

Zusammen

$\begin{array}{ll}5 & 0,2 \\ 4 & 0,0\end{array}$

Sonst. wissensch. Personal

$$
\begin{aligned}
& \text { männlich } \\
& \text { weiblich }
\end{aligned}
$$

Zusammen

$100 \quad 54,8$

$53 \quad 27,5$

15382,2

$2,4 \quad 11,6 \quad 23,3$

SUMME wissensch. Personal

$$
\text { männlich }
$$

weiblich

Zusammen

$\begin{array}{rrrr}334 & 155,5 & - & 17 \\ 147 & 60,4 & 2,4 & 14 \\ 481 & 216,0 & 2,4 & 32,\end{array}$

$\begin{array}{lr}- & - \\ - & 0,2 \\ - & 0,2\end{array}$

2,3
-
2,3

$\begin{array}{lll}0,8 & 2,6 & 5,4\end{array}$

5,4

$\begin{array}{lll}0,5 & 0,7 & 0,8 \\ 1,3 & 3,2 & 6,2\end{array}$

8,9

$\begin{array}{rr}2,4 & 3,5 \\ - & 0,3 \\ 2,4 & 3,8\end{array}$

2,4

17,3
14,9
32,1

42,3

21,9

38,3
9,1
47,4

19,5
4,4
23,9

10,5
3,4

3,4
13,9

8,8
2,6
11,3

13,0
1,6

14,6

2,4
-
2,4

3,5

0,3

3,8 
Tabelle 18 (Fortsetzung 4)

\section{0 bis 4.0 ZWISCHENSUMME}

\begin{tabular}{|c|c|c|c|c|c|c|c|c|c|c|c|c|}
\hline \multirow{4}{*}{ Beschäftigten-Kategorien } & \multicolumn{12}{|c|}{ Wissenschaftliches Personal insgesamt in } \\
\hline & \multirow{3}{*}{$\begin{array}{l}\text { Kopf- } \\
\text { zahlen }\end{array}$} & \multicolumn{11}{|c|}{ Vollzeitäquivalenten (VZÄ) für F\&E } \\
\hline & & \multirow{2}{*}{$\begin{array}{l}\text { INSGE- } \\
\text { SAMT }\end{array}$} & \multicolumn{10}{|c|}{ davon VZÄ für Beschäftigte im Alter von: } \\
\hline & & & $\begin{array}{c}\text { unter } 25 \\
\text { Jahren }\end{array}$ & $\begin{array}{c}25 \text { bis } 29 \\
\text { Jahre }\end{array}$ & $\begin{array}{c}30 \text { bis } 34 \\
\text { Jahre }\end{array}$ & $\begin{array}{c}35 \text { bis } 39 \\
\text { Jahre }\end{array}$ & $\begin{array}{c}40 \text { bis } 44 \\
\text { Jahre }\end{array}$ & $\begin{array}{c}45 \text { bis } 49 \\
\text { Jahre }\end{array}$ & $\begin{array}{c}50 \text { bis } 54 \\
\text { Jahre }\end{array}$ & $\begin{array}{c}55 \text { bis } 59 \\
\text { Jahre }\end{array}$ & $\begin{array}{c}60 \text { bis } 64 \\
\text { Jahre }\end{array}$ & $\begin{array}{l}65 \mathrm{~J} . \mathrm{u} . \\
\text { darüber }\end{array}$ \\
\hline
\end{tabular}

Professoren

männlich

weiblich

Zusammen

Universitätsdozenten

männlich

weiblich

Zusammen

Assistenten

$$
\text { männlich }
$$

weiblich

Zusammen

Wissenschaftliche Hilfskräfte

$$
\begin{aligned}
& \text { männlich } \\
& \text { weiblich }
\end{aligned}
$$$$
\text { Zusammen }
$$

Sonst. wissensch. Personal

$$
\begin{aligned}
& \text { männlich } \\
& \text { weiblich } \\
& \text { Zusammen }
\end{aligned}
$$

$$
1107
$$

53
160

456,5

$$
22,8
$$

$$
479,3
$$

$$
1220 \quad 517,0
$$

$\begin{array}{rr}1220 & 517,0 \\ 137 & 49,4\end{array}$

$\begin{array}{rr}137 & 49,4 \\ 1357 & 566,4\end{array}$

$\begin{array}{rr}3620 & 1224,2 \\ 1363 & 372,6\end{array}$

$1363 \quad 372,6$

1596,8

$106 \quad 16,7$

$2024 \quad 1120,5$

$\begin{array}{rr}2024 & 1120,5 \\ 777 & 406,2\end{array}$

$2801 \quad 1526,7$

25,2

20,3

45,5

188,2

627,7

115,2

474,6

29,2
22,6
51,8

644,8
255,8

811,4
242,4

242,4
1053,8

$2384 \quad 857,4$

900,6

1,0
2,3$$
\text { weiblich }
$$

10461

$\begin{array}{rr}54 & 6,3 \\ 160 & 23,0\end{array}$

$1,3 \quad 14,1$

14,1

2,2
16,3

35,2

2,7
37,9

51,3
4,1

4,1
55,4

84,7

3,1
87,9

151,2

4,6
155,8

60,9

2,1
63,0

$89,8 \quad 130,9$

101,1

9,7

110,8

100,8
7,6

7,6
108,4

66,4

4,7

14,7

14,7

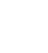

$\begin{array}{rr}145,2 & 72,7 \\ 42,1 & 15,7\end{array}$

42,1

15,7
88,4

47,3

13,6

387,9$$
1,0
$$

0,1

$5,3 \quad 1,5$
4192,3 
Tabelle 18 (Fortsetzung 5)

\subsection{SOZIALWISSENSCHAFTEN}

\begin{tabular}{|c|c|c|c|c|c|c|c|c|c|c|c|c|}
\hline \multirow{4}{*}{ Beschäftigten-Kategorien } & \multicolumn{12}{|c|}{ Wissenschaftliches Personal insgesamt in } \\
\hline & \multirow{3}{*}{$\begin{array}{l}\text { Kopf- } \\
\text { zahlen }\end{array}$} & \multicolumn{11}{|c|}{ Vollzeitäquivalenten (VZÄ) für F\&E } \\
\hline & & \multirow{2}{*}{$\begin{array}{l}\text { INSGE- } \\
\text { SAMT }\end{array}$} & \multicolumn{10}{|c|}{ davon VZÄ für Beschäftigte im Alter von: } \\
\hline & & & $\begin{array}{c}\text { unter } 25 \\
\text { Jahren }\end{array}$ & $\begin{array}{c}25 \text { bis } 29 \\
\text { Jahre }\end{array}$ & $\begin{array}{c}30 \text { bis } 34 \\
\text { Jahre }\end{array}$ & $\begin{array}{c}35 \text { bis } 39 \\
\text { Jahre }\end{array}$ & $\begin{array}{c}40 \text { bis } 44 \\
\text { Jahre }\end{array}$ & $\begin{array}{c}45 \text { bis } 49 \\
\text { Jahre }\end{array}$ & $\begin{array}{c}50 \text { bis } 54 \\
\text { Jahre }\end{array}$ & $\begin{array}{c}55 \text { bis } 59 \\
\text { Jahre }\end{array}$ & $\begin{array}{c}60 \text { bis } 64 \\
\text { Jahre }\end{array}$ & $\begin{array}{l}65 \mathrm{~J} . \mathrm{u} . \\
\text { darüber }\end{array}$ \\
\hline
\end{tabular}

Professoren

männlich

weiblich

Zusammen

$452 \quad 204,2$

$\begin{array}{rr}31 & 12,6 \\ 483 & 216,7\end{array}$

12,6

Universitätsdozenten

männlich

weiblich

Zusammen

$\begin{array}{rr}189 & 78,6 \\ 24 & 9,9 \\ 213 & 88,5\end{array}$

Assistenten

$$
\text { männlich }
$$

weiblich

Zusammen

$\begin{array}{rr}723 & 274 \\ 476 & 155 \\ 1199 & 429\end{array}$

$\begin{array}{lll}274,4 & 5,5 & 71,8 \\ 155,3 & 5,8 & 50,3\end{array}$

$429,7 \quad 11,2 \quad 122,0$

0,8
0,2
1,0

3,2

$\begin{array}{rr}7,2 & 17,5 \\ 1,1 & 2,0 \\ 8,3 & 19,6\end{array}$

24,5

44,0

$\begin{array}{rrr}62,8 & 26,9 & 17,2 \\ 2,0 & 0,5 & - \\ 64,8 & 27,4 & 17,2\end{array}$

Wissenschaftliche Hilfskräfte

$$
\begin{aligned}
& \text { männlich } \\
& \text { weiblich } \\
& \text { Zusammen }
\end{aligned}
$$

$\begin{array}{ll}46 & 4,4 \\ 42 & 5,0\end{array}$

88

5,0
9,4

1,2

1,2
3,5

1,5
1,2

1,2
2,7

$1,4 \quad 4,9$

4,9

14,8

16,9

27,1

4,2
48,2

64,8

27,4

17,2

Sonst. wissensch. Personal

$$
\begin{aligned}
& \text { männlich } \\
& \text { weiblich }
\end{aligned}
$$

Zusammen

$180 \quad 63,4$

$95 \quad 30,7$

27594,1

$0,5 \quad 17,9$

$\begin{array}{ll}0,8 & 10,6 \\ 1,3 & 28,5\end{array}$

$\begin{array}{rr}1590 & 625,0 \\ 668 & 213,5\end{array}$

625,0
213,5

$\begin{array}{rrr}7,2 & 93,4 & 110,8 \\ 8,9 & 62,3 & 53,5 \\ 16,1 & 155,7 & 164,3\end{array}$

86,7

17,0

0,4
17,3

14,5

14,8

$10,2 \quad 0,6$

0,5

SUMME wissensch. Personal

$$
\begin{aligned}
& \text { männlich } \\
& \text { weiblich }
\end{aligned}
$$

Zusammen

2258

838,5

16,1

155,7

53,5
164,3

83,9
37,8

37,8
121,7

51,0

29,9

16,4

3,4
18,2

1,1
11,3

0,5
1,1

0,5

15,1
6,6

10,5

2,9

3,7
1,3

0,3

0,3

$\begin{array}{rrr}1,6 & - & - \\ 1,2 & 0,1 & 0,2 \\ 2,8 & 0,1 & 0,2\end{array}$

-

$$
0,1
$$

0,1

$\begin{array}{rrr}14,4 & 10,9 & 3,2 \\ 6,4 & 3,6 & 1,7 \\ 20,7 & 14,5 & 5,0\end{array}$

3,2

2,1

$\begin{array}{ll}5,1 & 7,5 \\ 3,6 & 1,9\end{array}$

$\begin{array}{ll}3,6 & 1,9 \\ 8,8 & 9,4\end{array}$

0,7

0,7

10


Tabelle 18 (Fortsetzung 6)

\subsection{GEISTESWISSENSCHAFTEN}

\begin{tabular}{|c|c|c|c|c|c|c|c|c|c|c|c|c|}
\hline \multirow{4}{*}{ Beschäftigten-Kategorien } & \multicolumn{6}{|c|}{ Wissenschaftliches } & \multicolumn{3}{|c|}{ ersonal insgesam t in } & & & \\
\hline & \multirow{3}{*}{$\begin{array}{l}\text { Kopf- } \\
\text { zahlen }\end{array}$} & \multicolumn{11}{|c|}{ Vollzeitäquivalenten (VZÄ) für F\&E } \\
\hline & & \multirow{2}{*}{$\begin{array}{l}\text { INSGE- } \\
\text { SAMT }\end{array}$} & \multicolumn{10}{|c|}{ davon VZÄ für Beschäftigte im Alter von: } \\
\hline & & & $\begin{array}{c}\text { unter } 25 \\
\text { Jahren }\end{array}$ & $\begin{array}{c}25 \text { bis } 29 \\
\text { Jahre }\end{array}$ & $\begin{array}{c}30 \text { bis } 34 \\
\text { Jahre }\end{array}$ & $\begin{array}{c}35 \text { bis } 39 \\
\text { Jahre }\end{array}$ & $\begin{array}{c}40 \text { bis } 44 \\
\text { Jahre }\end{array}$ & $\begin{array}{c}45 \text { bis } 49 \\
\text { Jahre }\end{array}$ & $\begin{array}{c}50 \text { bis } 54 \\
\text { Jahre }\end{array}$ & $\begin{array}{c}55 \text { bis } 59 \\
\text { Jahre }\end{array}$ & $\begin{array}{c}60 \text { bis } 64 \\
\text { Jahre }\end{array}$ & $\begin{array}{l}65 \mathrm{~J} . \mathrm{u} . \\
\text { darüber }\end{array}$ \\
\hline
\end{tabular}

Professoren

männlich

weiblich

Zusammen

$313 \quad 133,9$

$39 \quad 15,7$

Universitätsdozenten

männlich

weiblich

Zusammen

$153 \quad 71,2$

15,7
49,6

en

männlich

weiblich

Zusammen

$\begin{array}{rr}153 & 71,2 \\ 36 & 15,4\end{array}$

$189 \quad 86,7$

86,7

$346 \quad 134,3$

$593 \quad 212,0$

$\begin{array}{rr}- & 9,2 \\ 0,4 & 10,8\end{array}$

0,4

10,8

$\begin{array}{lll}- & - & 1,1 \\ - & - & 0,7\end{array}$

$1,1 \quad 5,0$

5,0

$6,1-27,1$

48,9

24,3

21,4

Wissenschaftliche Hilfskräfte

$$
\begin{aligned}
& \text { männlich } \\
& \text { weiblich } \\
& \text { Zusammen }
\end{aligned}
$$

$\begin{array}{ll}13 & 1,2 \\ 17 & 1,5\end{array}$

Sonst. wissensch. Personal

$$
\begin{aligned}
& \text { männlich } \\
& \text { weiblich } \\
& \text { Zusammen }
\end{aligned}
$$

$259 \quad 72,1$

$447 \quad 138,0$

$\begin{array}{llll}0,6 & 0,1 & 0,2 & 0,4 \\ 0,2 & 0,6 & 0,2 & 0,4 \\ 0,8 & 0,7 & 0,4 & 0,8\end{array}$

0,1
0,1

SUMME wissensch. Personal

$$
\text { männlich }
$$

weiblich

Zusammen

$\begin{array}{rr}1013 & 406,6 \\ 598 & 182,4 \\ 1611 & 588,9\end{array}$

406,6
182,4
588,9

0,5

8,4

13,8

$17,6 \quad 17,0$

$19,0 \quad 10,5$

36,6

27,5

$6,7 \quad 3,3$

$\begin{array}{ll}6,7 & 3,3 \\ 9,1 & 8,0\end{array}$

9,1
15,8

11,3

3,7

6,0
9,7

4,0

4,0
11,9

0,9

1,4

0,3

$\begin{array}{llll} & & & \\ 1,1 & 18,1 & 41,8 & 52,7\end{array}$

\section{4,2}

38,9
80,7

52,7
30,5
83,2

54,2
28,5
82,7

44,0
22,8

61,5

61,5
14,4

$82,5 \quad 29,1$

$29,1 \quad 21,8$

$1,7 \quad 43,2$

43,2

66,8

75,8

99,2

33,1

0,8
22,6 
Tabelle 18 (Fortsetzung 7)

\section{0 und 6.0 ZWISCHENSUMME}

\begin{tabular}{|c|c|c|c|c|c|c|c|c|c|c|c|c|}
\hline \multirow{4}{*}{ Beschäftigten-Kategorien } & \multicolumn{12}{|c|}{ Wissenschaftliches Personal insgesamt in } \\
\hline & \multirow{3}{*}{$\begin{array}{l}\text { Kopf- } \\
\text { zahlen }\end{array}$} & \multicolumn{11}{|c|}{ Vollzeitäquivalenten (VZÄ) für F\&E } \\
\hline & & \multirow{2}{*}{$\begin{array}{l}\text { INSGE- } \\
\text { SAMT }\end{array}$} & \multicolumn{10}{|c|}{ davon VZÄ für Beschäftigte im Alter von: } \\
\hline & & & $\begin{array}{l}\text { unter } 25 \\
\text { Jahren }\end{array}$ & $\begin{array}{c}25 \text { bis } 29 \\
\text { Jahre }\end{array}$ & $\begin{array}{c}30 \text { bis } 34 \\
\text { Jahre }\end{array}$ & $\begin{array}{c}35 \text { bis } 39 \\
\text { Jahre }\end{array}$ & $\begin{array}{c}40 \text { bis } 44 \\
\text { Jahre }\end{array}$ & $\begin{array}{c}45 \text { bis } 49 \\
\text { Jahre }\end{array}$ & $\begin{array}{c}50 \text { bis } 54 \\
\text { Jahre }\end{array}$ & $\begin{array}{c}55 \text { bis } 59 \\
\text { Jahre }\end{array}$ & $\begin{array}{c}60 \text { bis } 64 \\
\text { Jahre }\end{array}$ & $\begin{array}{l}65 \mathrm{~J} . \mathrm{u} . \\
\text { darüber }\end{array}$ \\
\hline
\end{tabular}

Professoren

männlich

weiblich

Zusammen

$765 \quad 338,1$

$\begin{array}{rr}70 & 28, \\ 835 & 366,3\end{array}$

$\begin{array}{ll}- & 0,8\end{array}$

tsdozenten

männlich

weiblich

Zusammen

$342 \quad 149,8$

$60 \quad 25,3$

$402 \quad 175,1$

Assistenten

männlich

weiblich

Zusammen

$1069 \quad 408,7$

$408,7 \quad 5,5$

233,0

641,7

5,5
6,2

5,5
6,2
11,6

81,0

61,0

142,0

3,2
-

8,2
1,8
10,0

22,5

22,5
4,2
26,7

30,7

30,7
4,7
35,4

71,1

111,7

51,2

38,6

haftliche Hilfskräfte

$$
\text { männlich }
$$

weiblich

$\begin{array}{rr}59 & 5,6 \\ 59 & 6,5 \\ 118 & 12,1\end{array}$

$1,8 \quad 1,6$

1,6
1,8

4,3

3,4

$368 \quad 129,3$

männlich

weiblich

Zusammen

$354 \quad 102,8$

$722 \quad 232,1$

1,0
0,8
1,8

$\begin{array}{lll}26,3 & 31,9 & 27,9\end{array}$

$26,3 \quad 31,9$

$\begin{array}{ll}24,4 & 25,4 \\ 50,7 & 57,3\end{array}$

14,1

41,9

10,8

10,8
20,7

$\begin{array}{rr}2603 & 1031,6 \\ 1266 & 395,8\end{array}$

männlich

weiblich

Zusammen

3869

395,8
1427,4

8,2
9,6
17,8

$\begin{array}{rr}111,4 & 152,6 \\ 87,4 & 92,4 \\ 198,9 & 244,9\end{array}$

136,6
68,3

68,3
204,9

121,7
44,9
166,6

101,3
35,9

35,9
137,2

28,5

164,4

23,0

189,7

5,0
62,6 
Tabelle 18 (Fortsetzung 8 und Schluss)

1.0 bis 6.0 INSGESAMT

\begin{tabular}{|c|c|c|c|c|c|c|c|c|c|c|c|c|}
\hline \multirow{4}{*}{ Beschäftigten-Kategorien } & \multicolumn{12}{|c|}{ Wissenschaftliches Personal insgesamt } \\
\hline & \multirow{3}{*}{$\begin{array}{l}\text { Kopf- } \\
\text { zahlen }\end{array}$} & \multicolumn{11}{|c|}{ Vollzeitäquivalenten (VZÄ) für F\&E } \\
\hline & & \multirow{2}{*}{$\begin{array}{l}\text { INSGE- } \\
\text { SAMT }\end{array}$} & \multicolumn{10}{|c|}{ davon VZÄ für Beschäftigte im Alter von: } \\
\hline & & & $\begin{array}{c}\text { unter } 25 \\
\text { Jahren }\end{array}$ & $\begin{array}{c}25 \text { bis } 29 \\
\text { Jahre }\end{array}$ & $\begin{array}{c}30 \text { bis } 34 \\
\text { Jahre }\end{array}$ & $\begin{array}{c}35 \text { bis } 39 \\
\text { Jahre }\end{array}$ & $\begin{array}{c}40 \text { bis } 44 \\
\text { Jahre }\end{array}$ & $\begin{array}{c}45 \text { bis } 49 \\
\text { Jahre }\end{array}$ & $\begin{array}{c}50 \text { bis } 54 \\
\text { Jahre }\end{array}$ & $\begin{array}{c}55 \text { bis } 59 \\
\text { Jahre }\end{array}$ & $\begin{array}{c}60 \text { bis } 64 \\
\text { Jahre }\end{array}$ & $\begin{array}{l}65 \mathrm{~J} . \mathrm{u} . \\
\text { darüber }\end{array}$ \\
\hline
\end{tabular}

Professoren

männlich

weiblich

Zusammen

Universitätsdozenten

$$
\begin{aligned}
& \text { männlich } \\
& \text { weiblich } \\
& \text { Zusammen }
\end{aligned}
$$

Assistenten

$$
\begin{aligned}
& \text { männlich } \\
& \text { weiblich }
\end{aligned}
$$

Zusammen

Wissenschaftliche Hilfskräfte

$$
\begin{aligned}
& \text { männlich } \\
& \text { weiblich } \\
& \text { Zusammen }
\end{aligned}
$$

Sonst. wissensch. Personal

$$
\begin{aligned}
& \text { männlich } \\
& \text { weiblich } \\
& \text { Zusammen }
\end{aligned}
$$

1872
123
1995

\section{4,6}

$$
\begin{array}{r}
51,0 \\
845,6
\end{array}
$$

1562

197
1759

\section{6,8}$$
\begin{array}{r}
74,7 \\
741,6
\end{array}
$$

\section{$4689 \quad 1632,9$}

$\begin{array}{rr}4689 & 1632,9 \\ 2086 & 605,6\end{array}$

$6775 \quad 2238,6$

Jahre

ren

SUMME wissensch. Personal

$$
\begin{aligned}
& \text { männlich } \\
& \text { weiblich } \\
& \text { Zusammen }
\end{aligned}
$$

165
113
278

$\quad 14,4$

2,0
0,2
2,2

4,5
1,0
5,5

22,4
3,9
26,3

57,8
6,9
64,6

82,0
8,8
90,8

155,8
9,6
165,4

262,9
13,8
276,7

$\begin{array}{rr}112,2 & 95,2 \\ 3,3 & 3,6 \\ 115,5 & 98,7\end{array}$

- $\quad 2,0$

16,2

109,7

158,5

128,6

165,4

276,7

115,5

3,6
98,7

2,0

1,9
18,1

14,7
124,4

158,5
19,5

128,6

138,0

138,0
14,9

$93,4 \quad 17,4$

$17,4 \quad 3,0$

2,0

\section{4,9}

$124,4 \quad 178,0$

143,6

152,9

100,7

1,5
18,9

3,0

7,7
6,8
14,4

$\begin{array}{lll}278,3 & 544,9 & 369,4\end{array}$

$\begin{array}{ll}369,4 & 206,9\end{array}$

$147,0 \quad 67,4$

109,4

31,5

734,4

516,4

274,2

140,8

66,0

18,8

84,9

\section{0,9}

40,9
16,7

57,6

9,0
3,0

3,0
12,1

22,3
12,8
35,1

3,6
4,2
7,8

8,2

$7,1 \quad 1,8$

$1,8 \quad 1,0$

$\begin{array}{rr}0,1 & 0,5 \\ - & - \\ 0,1 & 0,5\end{array}$

$2392 \quad 1249,8$

$\begin{array}{lr}1131 & 509,0\end{array}$

$26,2 \quad 465,8 \quad 391,3$

$26,2 \quad 465,8 \quad 391,3$

$\begin{array}{lll}21,1 & 212,6 & 140,6 \\ 47,3 & 678,4 & 531,9\end{array}$

177,6
43,6

221,2

$\begin{array}{lll}60,7 & 31,1 & 35,8 \\ 30,2 & 26,4 & 17,6\end{array}$

$\begin{array}{lll}30,2 & 26,4 & 17,6 \\ 90,9 & 57,5 & 53,4\end{array}$

48,4

12,4

60,7

13,0

4,2
17,2

0,3

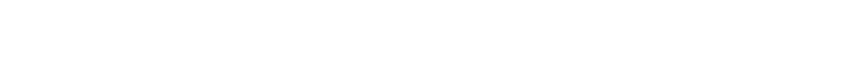

$\begin{array}{rr}10680 & 4366,5 \\ 3650 & 1253,2 \\ 14330 & 5619,6\end{array}$

$\begin{array}{rrr}37,5 & 756,2 & 964,0 \\ 32,1 & 343,2 & 334,8 \\ 69,6 & 1099,4 & 1298,8\end{array}$

680,9

$\begin{array}{ll}680,9 & 484,8 \\ 210,2 & 124,2\end{array}$

$891,0 \quad 609,0$

351,1
81,7

396,2
60,9

445,6

445,6
50,2

495,7

151,6

$\begin{array}{rr}151,6 & 98,7 \\ 12,1 & 3,8\end{array}$

$\begin{array}{rr}163,7 & 3,8 \\ 102,6\end{array}$ 
Tabelle 19: FORSCHUNG UND EXPERIMENTELLE ENTWICKLUNG IN SÄMTLICHEN ERHEBUNGSBEREICHEN BESCHÄFTIGTE in F\&E (in Kopfzahlen und in Vollzeitäquivalent) im Jahre 1998

gegliedert nach Beschäftigtenkategorien

\begin{tabular}{|c|c|c|c|c|c|c|c|c|c|c|}
\hline & \multirow[b]{3}{*}{ Sektoren/Bereiche } & \multirow{3}{*}{$\begin{array}{c}\text { Anzahl der } \\
F \& E \text { betrei- } \\
\text { benden Er- } \\
\text { hebungsein- } \\
\text { heiten }\end{array}$} & \multicolumn{4}{|c|}{ Kopfzahlen } & \multicolumn{4}{|c|}{ Vollzeitäquivalente für F\&E } \\
\hline & & & \multirow[b]{2}{*}{ INSGESAMT } & \multicolumn{3}{|c|}{ davon: } & \multirow[b]{2}{*}{ INSGESAMT } & \multicolumn{3}{|c|}{ davon: } \\
\hline & & & & $\begin{array}{l}\text { Wissenschaft- } \\
\text { liches Personal }\end{array}$ & $\begin{array}{c}\text { Höherqualifiziertes } \\
\text { nichtwissenschaft- } \\
\text { liches Personal } \\
\text { (Maturanten, Tech- } \\
\text { niker, Laboranten) }\end{array}$ & $\begin{array}{l}\text { Sonstiges } \\
\text { Hilfspersonal }\end{array}$ & & $\begin{array}{l}\text { Wissenschaft- } \\
\text { liches Personal }\end{array}$ & $\begin{array}{l}\text { Höherqualifiziertes } \\
\text { nichtwissenschaft- } \\
\text { liches Personal } \\
\text { (Maturanten, Tech- } \\
\text { niker, Laboranten) }\end{array}$ & $\begin{array}{l}\text { Sonstiges } \\
\text { Hilfspersonal }\end{array}$ \\
\hline \multirow[t]{6}{*}{1.} & $\begin{array}{l}\text { Hochschulsektor } \\
\text { davon: }\end{array}$ & 1015 & 21933 & 14960 & 3265 & 3708 & 8670,1 & 5955,3 & 1242,1 & 1472,8 \\
\hline & 1.1 Universitäten & 822 & 16291 & 11115 & 2294 & 2882 & 6963,3 & 4801,5 & 919,0 & 1242,8 \\
\hline & 1.2 Universitätskliniken & 77 & 4810 & 3215 & 854 & 741 & 1277,7 & 822,0 & 271,6 & 184,1 \\
\hline & 1.3 Universitäten der Künste & 56 & 239 & 210 & 9 & 20 & 74,0 & 63,5 & 3,4 & 7,0 \\
\hline & 1.4 Akademie der Wissenschaften & 56 & 582 & 412 & 105 & 65 & 351,6 & 265,5 & 47,3 & 38,8 \\
\hline & 1.5 Versuchsanstalten an HTLs & 4 & 11 & 8 & 3 & - & 3,5 & 2,8 & 0,8 & - \\
\hline 2. & Sektor Staat & 358 & 5734 & 2290 & 1164 & 2280 & 2104,4 & 954,0 & 325,5 & 824,9 \\
\hline 3. & Privater gemeinnütziger Sektor & 53 & 349 & 188 & 92 & 69 & 148,4 & 89,5 & 32,8 & 26,2 \\
\hline \multirow[t]{4}{*}{4.} & $\begin{array}{l}\text { Unternehmenssektor } \\
\text { davon: }\end{array}$ & 1317 & 24940 & 13966 & 7993 & 2981 & 20384,6 & 11716,1 & 6318,7 & 2349,9 \\
\hline & 4.1 Kooperativer Bereich & 25 & 2915 & 1217 & 864 & 834 & 1834,4 & 773,0 & 497,8 & 563,6 \\
\hline & $\begin{array}{l}\text { 4.2 Ziviltechniker } \\
4.3 \text { Kraftwerksaesellschaften ') }\end{array}$ & 20 & 130 & 52 & 33 & 45 & 23,2 & 12,3 & 4,3 & 6,7 \\
\hline & 4.4 Firmeneigener Bereich & $1272^{\circ}$ & $21895^{\circ}$ & 12697 & 7096 & 2102 & 18527,0 & 10930,8 & 5816,6 & 1779,6 \\
\hline \multicolumn{2}{|c|}{ INSGESAMT } & 2743 & 52956 & 31404 & 12514 & 9038 & 31307,5 & 18714,9 & 7919,1 & 4673,8 \\
\hline
\end{tabular}

Stand: April 2001

Quelle: Statistik Austria (Bundesanstalt Statistik Österreich)

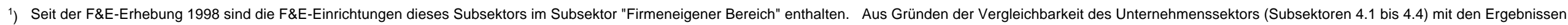
aus früheren F\&E-Erhebungen wurde der Subsektor "Kraftwerksgesellschaften" in der Vorspalte weiter angeführt. -- Rundungsdifferenzen. 
Tabelle 20: FORSCHUNG UND EXPERIMENTELLE ENTWICKLUNG IN SÄMTLICHEN ERHEBUNGSBEREICHEN BESCHÄFTIGTE in F\&E (in Kopfzahlen und in Vollzeitäquivalent) im Jahre 1998 gegliedert nach Beschäftigtenkategorien und Geschlecht

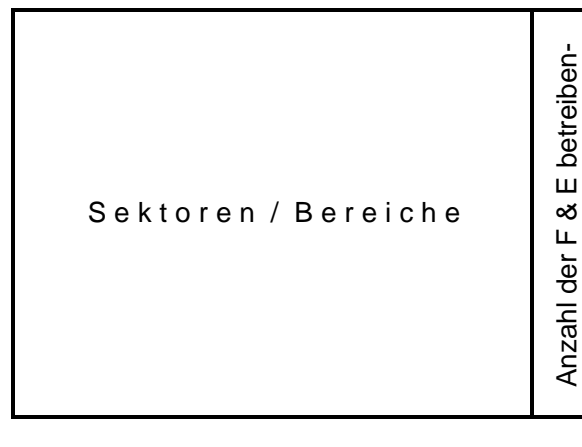

1. Hochschulsektor

davon:

1.1 Universitäten

1.2 Universitätskliniken

1.3 Universitäten der Künste

1.4 Akademie der Wissenschaften

1.5 Versuchsanstalten an HTLs

2. Sektor Staat

3. Privater gemeinnütziger Sektor

4. Unternehmenssektor

davon:

4.1 Kooperativer Bereich

4.2 Ziviltechniker

4.3 Kraftwerksgesellschaften ')

4.4 Firmeneigener Bereich

INSGESAMT
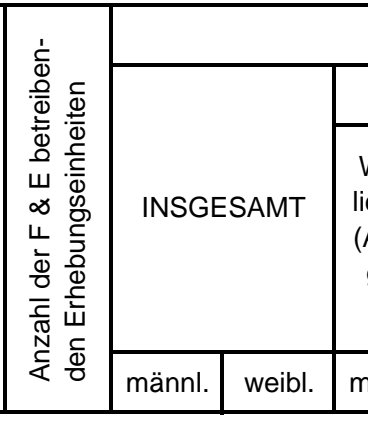

\begin{tabular}{|c|c|c|c|c|c|}
\hline \multicolumn{6}{|c|}{ Kopfzahlen } \\
\hline \multicolumn{6}{|c|}{ davon: } \\
\hline \multicolumn{2}{|c|}{$\begin{array}{l}\text { Wissenschaft- } \\
\text { liches Personal } \\
\text { (Akademiker u. } \\
\text { gleichwertige } \\
\text { Kräfte) }\end{array}$} & \multicolumn{2}{|c|}{$\begin{array}{l}\text { Höherqualifiziertes } \\
\text { nichtwissenschaft- } \\
\text { liches Personal } \\
\text { (Maturanten, Tech- } \\
\text { niker, Laboranten) }\end{array}$} & \multicolumn{2}{|c|}{$\begin{array}{l}\text { Sonstiges } \\
\text { Hilfspersonal }\end{array}$} \\
\hline männl. & weibl. & männl. & weibl. & männl. & weibl. \\
\hline
\end{tabular}

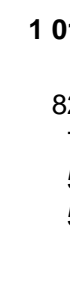

$\begin{array}{rrrrrr}\mathbf{1 0 1 5} & \mathbf{1 3} \mathbf{3 5 1} & \mathbf{8 5 8 2} & \mathbf{1 1} \mathbf{1 1 8} & \mathbf{3} \mathbf{8 4 2} & \mathbf{1} \\ \mathbf{8 2 2} & 10400 & 5891 & 8478 & 2637 & \\ 77 & 2439 & 2371 & 2202 & 1013 & \\ 56 & 149 & 90 & 145 & 65 & \\ 56 & 354 & 228 & 286 & 126 & \\ 4 & 9 & 2 & 7 & 1\end{array}$

$\begin{array}{lllll}358 & 3283 & 2451 & 1560 & 730\end{array}$

$\begin{array}{lllll}53 & 161 & 188 & 117 & 71\end{array}$

$\begin{array}{lllll}1317 & 21103 & 3837 & 12708 & 1258\end{array}$

$\begin{array}{llll}25 & 2173 & 742 & 1033\end{array}$

20

$1272 \quad 18850 \quad 3045 \quad 11634$

$184 \quad 687$

weibl.

\begin{tabular}{|c|c|c|c|c|c|}
\hline \multicolumn{6}{|c|}{ davon: } \\
\hline \multicolumn{2}{|c|}{$\begin{array}{l}\text { Wissenschaft- } \\
\text { liches Personal } \\
\text { (Akademiker u. } \\
\text { gleichwertige } \\
\text { Kräfte) }\end{array}$} & \multicolumn{2}{|c|}{$\begin{array}{l}\text { Höherqualifiziertes } \\
\text { nichtwissenschaft- } \\
\text { liches Personal } \\
\text { (Maturanten, Tech- } \\
\text { niker, Laboranten) }\end{array}$} & \multicolumn{2}{|c|}{$\begin{array}{l}\text { Sonstiges } \\
\text { Hilfspersonal }\end{array}$} \\
\hline männl. & weibl. & männl. & weibl. & männl. & weibl. \\
\hline
\end{tabular}

$2743 \quad 37898 \quad 15058 \quad 25503 \quad 5901$
687
25

5946

$\begin{array}{rr}2 & \\ 2 & 1362 \\ 3 & 724 \\ 7 & \\ 2 & \end{array}$

$151 \quad 1119 \quad 2589 \quad 5570,0$

8420

618
30

362
724
6
58
1

$990 \quad 1892 \quad 4644,7 \quad 2318,7 \quad 3802,4$

$\begin{array}{lllll}107 & 634 & 642,8 & 634,9 & 566,6\end{array}$

$\begin{array}{lllll}1 & 19 & 45,7 & 28,3 & 44,2\end{array}$

$\begin{array}{llll}45,7 & 28,3 & 44,2 & 19,3\end{array}$

3,2

0,3

95,5
2,6

0,2

$\begin{array}{llll}546 & 1105 & 1175 & 1255,3\end{array}$

$849,1 \quad 665,0$

289,0

31,7

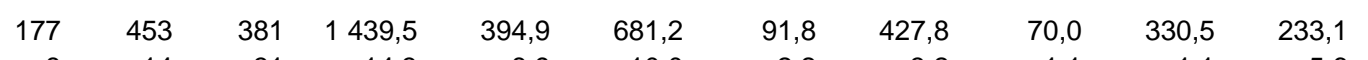

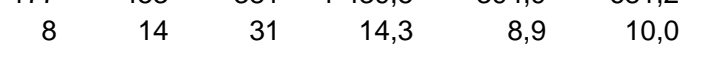

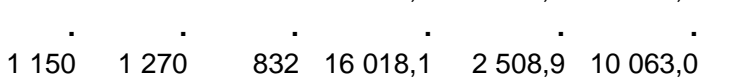

. 3,2

$867, \dot{8} \quad 4878, \dot{2} \quad 938, \dot{4} \quad 1076, \dot{9} \quad 702, \dot{7}$

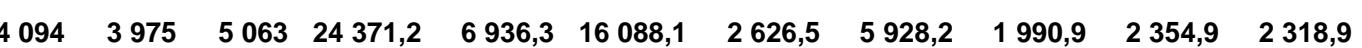

$192589 \quad 5570,0 \quad 3100,1 \quad 4611,2 \quad 1344,0$

$961,8 \quad 5309,2 \quad 1009,5 \quad 1408,5$

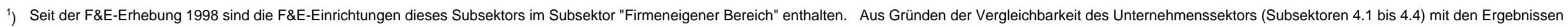
aus früheren F\&E-Erhebungen wurde der Subsektor "Kraftwerksgesellschaften" in der Vorspalte weiter angeführt. -- Rundungsdifferenzen. 
Tabelle 21: HOCHSCHULSEKTOR ${ }^{1}$ ): BESCHÄFTIGTE in FORSCHUNG und EXPERIMENTELLER ENTWICKLUNG (in Vollzeitäquivalent) im Jahre 1998 gegliedert nach Wissenschaftszweigen und Beschäftigtenkategorien

\begin{tabular}{|c|c|c|c|c|c|c|c|}
\hline \multirow[b]{2}{*}{ Wissenschaftszweige } & \multirow[b]{2}{*}{$\begin{array}{l}\text { Anzahl der F\&E } \\
\text { betreibenden } \\
\text { Erhebungs- } \\
\text { einheiten }\end{array}$} & \multirow[b]{2}{*}{ Kopfzahlen } & \multirow[b]{2}{*}{$\begin{array}{c}\text { Vollzeit- } \\
\text { äquivalente } \\
\text { insgesamt }\end{array}$} & \multicolumn{4}{|c|}{ Vollzeitäquivalente für F\&E } \\
\hline & & & & $\begin{array}{l}\text { Wissenschaft- } \\
\text { liches Personal } \\
\text { (Akademiker u. } \\
\text { gleichwertige } \\
\text { Kräfte) }\end{array}$ & $\begin{array}{l}\text { Höherqualifiziertes } \\
\text { nichtwissenschaft- } \\
\text { liches Personal } \\
\text { (Maturanten, Tech- } \\
\text { niker, Laboranten) }\end{array}$ & $\begin{array}{l}\text { Sonstiges } \\
\text { Hilfspersonal }\end{array}$ & INSGESAMT \\
\hline
\end{tabular}

1.0 Naturwissenschaften

2.0 Technische Wissenschaften

3.0 Humanmedizin ohne Kliniken Kliniken einschließlich Kliniken

4.0 Land- u. Forstwirtschaft, Veterinärmedizin

1.0 bis 4.0

$$
\begin{array}{r}
\text { Zwischensumme } \\
\text { ohne Kliniken } \\
\text { einschließlich Kliniken }
\end{array}
$$

5.0 Sozialwissenschaften

6.0 Geisteswissenschaften

\section{0 und 6.0}

1.0 bis 6.0

\section{Zwischensumme}

INSGESAMT

ohne Kliniken

einschließlich Kliniken

$\begin{array}{rl}212 & 5681 \\ 177 & 281 \\ 75 & 2187 \\ 77 & 4810 \\ 152 & 6997\end{array}$

47

\section{1}

588

198

229

427

938

1015

5681

2812

2187

4810

6997

971

$\begin{array}{rr}11651 & 9120,4 \\ 16461 & 13238,9 \\ 3029 & 2263,3 \\ 2443 & 1908,6 \\ 5472 & 4171,8 \\ & \\ 17123 & 13292,2 \\ 21933 & 17410,7\end{array}$

Stand: April 2001

\section{1,0}

2191,4

1757,0

4118,5

5875,5

811,0

1950,5

817,9

545,5

822,0

1367,5

216,3

3530,2
4352,1
875,6
727,5
1603,1
5133,3
5955,3

341,1

440,3

2731,9

143,7

249,3

1210,9

1007,3

1277,7

2285,0

$\begin{array}{lll}271,6 & 184,1 & 1277,7 \\ 537,2 & 380,3 & 2285,0\end{array}$

$92,2 \quad 118,4 \quad 426,9$

426,9

5376,9

6654,6

1115,4

900,1

2015,5

7392,4

8670,1

$\left.{ }^{1}\right)$ Umfaßt Universitäten einschließlich Kliniken, Universitäten der Künste, Akademie der Wissenschaften und Versuchsanstalten an den Höheren Technischen Bundeslehranstalten. -- Rundungsdifferenzen 
Tabelle 22: SEKTOR STAAT ${ }^{1}$ ): BESCHÄFTIGTE in FORSCHUNG und EXPERIMENTELLER ENTWICKLUNG (in Vollzeitäquivalent) im Jahre 1998 gegliedert nach Wissenschaftszweigen und Beschäftigtenkategorien

\begin{tabular}{|c|c|c|c|c|c|c|c|}
\hline \multirow[b]{2}{*}{ Wissenschaftszweige } & \multirow[b]{2}{*}{$\begin{array}{l}\text { Anzahl der F\&E } \\
\text { betreibenden } \\
\text { Erhebungs- } \\
\text { einheiten }\end{array}$} & \multirow[b]{2}{*}{ Kopfzahlen } & \multirow[b]{2}{*}{$\begin{array}{l}\text { Vollzeit- } \\
\text { äquivalente } \\
\text { insgesamt }\end{array}$} & \multicolumn{4}{|c|}{ Vollzeitäquivalente für F\&E } \\
\hline & & & & $\begin{array}{l}\text { Wissenschaft- } \\
\text { liches Personal } \\
\text { (Akademiker u. } \\
\text { gleichwertige } \\
\text { Kräfte) }\end{array}$ & $\begin{array}{l}\text { Höherqualifiziertes } \\
\text { nichtwissenschaft- } \\
\text { liches Personal } \\
\text { (Maturanten, Tech- } \\
\text { niker, Laboranten) }\end{array}$ & $\begin{array}{l}\text { Sonstiges } \\
\text { Hilfspersonal }\end{array}$ & INSGESAMT \\
\hline
\end{tabular}

1.0 Naturwissenschaften

2.0 Technische Wissenschaften

3.0 Humanmedizin

4.0 Land- u. Forstwirtschaft, Veterinärmedizin

\section{0 bis 4.0}

Zwischensumme

5.0 Sozialwissenschaften

6.0 Geisteswissenschaften

5.0 und 6.0

1.0 bis 6.0

Zwischensumme

INSGESAMT

Stand: April 2001

$\begin{array}{rrrrrrr}39 & 877 & 745,6 & 126,5 & 30,3 & 123,1 & 279,9 \\ 26 & 510 & 452,0 & 81,2 & 34,4 & 73,1 & 188,6 \\ 79 & 448 & 268,1 & 86,3 & 32,4 & 22,4 & 141,1 \\ 26 & 1469 & 1334,8 & 112,7 & 70,1 & 253,8 & 436,6 \\ 170 & \mathbf{3} 304 & \mathbf{2 8 0 0 , 4} & \mathbf{4 0 6 , 6} & \mathbf{1 6 7 , 2} & \mathbf{4 7 2 , 5} & \mathbf{1 0 4 6 , 2} \\ 102 & 990 & 717,6 & 277,2 & 85,2 & \mathbf{9 1 , 4} & \mathbf{4 5 3 , 8} \\ 86 & 1440 & 1257,3 & 270,3 & \mathbf{7 3 , 1} & \mathbf{2 6 1 , 0} & \mathbf{6 0 4 , 4} \\ \mathbf{1 8 8} & \mathbf{2 4 3 0} & \mathbf{1 9 7 4 , 9} & \mathbf{5 4 7 , 4} & \mathbf{1 5 8 , 3} & \mathbf{3 5 2 , 4} & \mathbf{1 0 5 8 , 2} \\ \mathbf{3 5 8} & \mathbf{5 7 3 4} & \mathbf{4 7 7 5 , 2} & \mathbf{9 5 4 , 0} & \mathbf{3 2 5 , 5} & \mathbf{8 2 4 , 9} & \mathbf{2} \mathbf{1 0 4 , 4}\end{array}$

Quelle: Statistik Austria (Bundesanstalt Statistik Osterreich)

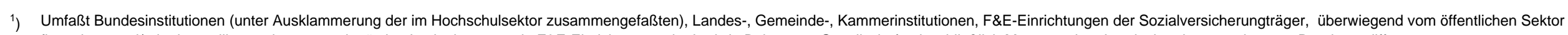
finanzierte und/oder kontrollierte private gemeinnützige Institutionen sowie F\&E-Einrichtungen der Ludwig Boltzmann-Gesellschaft; einschließlich Museen; ohne Landeskrankenanstalten. -- Rundungsdifferenzen. 
SEKTOR STAAT ${ }^{1}$ ): BESCHÄFTIGTE in FORSCHUNG und EXPERIMENTELLER ENTWICKLUNG (in Vollzeitäquivalent) im Jahre 1998 gegliedert nach Rechtsträgern und Beschäftigtenkategorien

\begin{tabular}{|c|c|c|c|c|c|c|c|}
\hline \multirow[b]{2}{*}{ Rechtsträger } & \multirow[b]{2}{*}{$\begin{array}{l}\text { Anzahl der F\&E } \\
\text { betreibenden } \\
\text { Erhebungs- } \\
\text { einheiten }\end{array}$} & \multirow[b]{2}{*}{ Kopfzahlen } & \multirow[b]{2}{*}{$\begin{array}{c}\text { Vollzeit- } \\
\text { äquivalente } \\
\text { insgesamt }\end{array}$} & \multicolumn{4}{|c|}{ Vollzeitäquivalente für F\&E } \\
\hline & & & & $\begin{array}{c}\text { Wissenschaft- } \\
\text { liches Personal } \\
\text { (Akademiker u. } \\
\text { gleichwertige } \\
\text { Kräfte) }\end{array}$ & $\begin{array}{l}\text { Höherqualifiziertes } \\
\text { nichtwissenschaft- } \\
\text { liches Personal } \\
\text { (Maturanten, Tech- } \\
\text { niker, Laboranten) }\end{array}$ & $\begin{array}{l}\text { Sonstiges } \\
\text { Hilfspersonal }\end{array}$ & INSGESAMT \\
\hline
\end{tabular}

Bund

Länder (einschließlich Wien)

Gemeinden (ohne Wien)

Kammern $^{2}$ )

Sozialversicherungsträger ${ }^{2}$ )

PloE öffentlich ${ }^{3}$ )

Ludwig Boltzmann-Gesellschaft
71

42

7

$\begin{array}{ll}7 & 2\end{array}$

.$\left.^{2}\right)$

123

108

358
2926

898

82

$43^{2}$ )

. ${ }^{2}$ )

1315

470

5734
2710,2

764,0

75,7

$39,0^{2}$ )

. ${ }^{2}$ )

955,3

231,0

4775,2
362,4

101,0

14,0

$8,4^{2}$ )

- $\left.{ }^{2}\right)$

344,7

123,7

954,0

325,5
499,2

165,2

18,2

$6,9^{2}$ )

${ }^{2}$ )

110,8

24,7

. ${ }^{2}$ )

12,6

824,9

2 104,4

Stand: April 2001

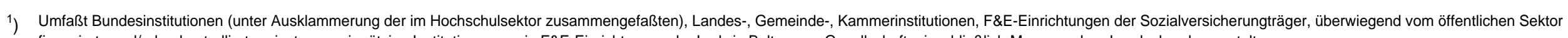
finanzierte und/oder kontrollierte private gemeinnützige Institutionen sowie F\&E-Einrichtungen der Ludwig Boltzmann-Gesellschaft; einschließlich Museen; ohne Landeskrankenanstalten.

2) Aus Geheimhaltungsgründen können die Daten nur gemeinsam ausgewiesen werden.

3) Private Institutionen ohne Erwerbscharakter, die überwiegend vom öffentlichen Sektor finanziert und/oder kontrolliert werden.

Rundungsdifferenzen. 
Tabelle 24: PRIVATER GEMEINNÜTZIGER SEKTOR ${ }^{1}$ ): BESCHÄFTIGTE in FORSCHUNG und EXPERIMENTELLER ENTWICKLUNG (in Vollzeitäquivalent) im Jahre 1998

gegliedert nach Wissenschaftszweigen und Beschäftigtenkategorien

\begin{tabular}{|c|c|c|c|c|c|c|c|}
\hline \multirow[b]{2}{*}{ Wissenschaftszweige } & \multirow[b]{2}{*}{$\begin{array}{l}\text { Anzahl der F\&E } \\
\text { betreibenden } \\
\text { Erhebungs- } \\
\text { einheiten }\end{array}$} & \multirow[b]{2}{*}{ Kopfzahlen } & \multirow[b]{2}{*}{$\begin{array}{l}\text { Vollzeit- } \\
\text { äquivalente } \\
\text { insgesamt }\end{array}$} & \multicolumn{4}{|c|}{ Vollzeitäquivalente für F\&E } \\
\hline & & & & $\begin{array}{l}\text { Wissenschaft- } \\
\text { liches Personal } \\
\text { (Akademiker u. } \\
\text { gleichwertige } \\
\text { Kräfte) }\end{array}$ & $\begin{array}{c}\text { Höherqualifiziertes } \\
\text { nichtwissenschaft- } \\
\text { liches Personal } \\
\text { (Maturanten, Tech- } \\
\text { niker, Laboranten) }\end{array}$ & $\begin{array}{l}\text { Sonstiges } \\
\text { Hilfspersonal }\end{array}$ & INSGESAMT \\
\hline
\end{tabular}

1.0 Naturwissenschaften

2.0 Technische Wissenschaften

3.0 Humanmedizin ${ }^{2}$ )

4.0 Land- u. Forstwirtschaft,

Veterinärmedizin ${ }^{2}$ )

\section{0 bis $4.0 \quad$ Zwischensumme}

5.0 Sozialwissenschaften

6.0 Geisteswissenschaften

5.0 und 6.0

Zwischensumme

INSGESAMT

1.0 bis 6.0

\section{1}

10

$4^{2}$ )

$\left.{ }^{2}\right)$

25

23

5

28

53

\section{1}

38

$72^{2}$ )

$\cdot^{2}$ )

181

156

12

168

349
41,4

23,0

$55,7^{2}$ )

. ${ }^{2}$ )

120,1

103,0

7,2

110,1

230,2
19,4

8,1

$16,0^{2}$ )

. ${ }^{2}$ )

43,5

42,5

3,5

46,0

89,5

\section{3,1}

1,4

$16,3^{2}$ )

. $\left.{ }^{2}\right)$

20,7

11,9

0,2

12,1

32,8
3,8

1,0

$8,5^{2}$ )

${ }^{2}$ )

13,3

12,7

0,2

12,9

26,2
26,3

10,5

$40,6^{2}$ )

. ${ }^{2}$ )

77,5

67,0

70,9

148,4

Stand: April 2001

Quelle: Statistik Austria (Bundesanstalt Statistik Osterreich)

1) F\&E-Einrichtungen, deren Status ein vorwiegend privater oder privatrechtlicher, konfessioneller oder sonstiger nicht öffentlicher ist.

2) Aus Geheimhaltungsgründen können die Daten nur gemeinsam ausgewiesen werden.

Rundungsdifferenzen 
Tabelle 25: UNTERNEHMENSSEKTOR OHNE FIRMENEIGENER BEREICH ${ }^{1}$ ):

KOOPERATIVER BEREICH ${ }^{2}$ ) und BEREICH der ZIVILTECHNIKER

BESCHÄFTIGTE in FORSCHUNG und EXPERIMENTELLER ENTWICKLUNG (in Vollzeitäquivalent) im Jahre 1998

gegliedert nach Wissenschaftszweigen und Beschäftigtenkategorien

\begin{tabular}{|c|c|c|c|c|c|c|c|c|}
\hline \multirow[b]{2}{*}{ Wissenschaftszweige } & \multicolumn{3}{|c|}{$\begin{array}{l}\text { Anzahl der F\&E betreibenden } \\
\text { Erhebungseinheiten }\end{array}$} & \multirow[b]{2}{*}{$\begin{array}{l}\text { Vollzeit- } \\
\text { äquivalente } \\
\text { insgesamt }\end{array}$} & \multicolumn{4}{|c|}{ Vollzeitäquivalente für F\&E } \\
\hline & 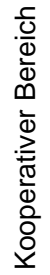 & 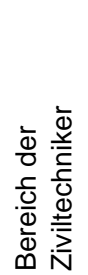 & $\sum_{\substack{\infty \\
N}}^{\substack{\infty \\
N}}$ & & $\begin{array}{l}\text { Wissenschaft- } \\
\text { liches Personal } \\
\text { (Akademiker u. } \\
\text { gleichwertige } \\
\text { Kräfte) }\end{array}$ & $\begin{array}{l}\text { Höherqualifiziertes } \\
\text { nichtwissenschaft- } \\
\text { liches Personal } \\
\text { (Maturanten, Tech- } \\
\text { niker, Laboranten) }\end{array}$ & $\begin{array}{l}\text { Sonstiges } \\
\text { Hilfspersonal }\end{array}$ & INSGESAMT \\
\hline
\end{tabular}

1.0 Naturwissenschaften

2.0 Technische Wissenschaften

3.0 Humanmedizin ${ }^{3}$ )

4.0 Land- u. Forstwirtschaft, Veterinärmedizin ${ }^{3}$ )

\section{4}

14

$\left.5^{3}\right)$

$\left.\cdot{ }^{3}\right)$

23

$2^{3}$ )

5.0 Sozialwissenschaften ${ }^{3}$ )

6.0 Geisteswissenschaften ${ }^{3}$ )

5.0 und 6.0

Zwischensumme

INSGESAMT

1.0 bis 6.0

2

\section{3}

15

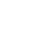

18

\section{7}

29

$\left.5^{3}\right)$

$\left.\cdot^{3}\right)$
591,6

1736,8

$247,4^{3}$ )

$\left.\cdot^{3}\right)$

\section{7,0}

479,9

$\left.88,1^{3}\right)$

$\left.\cdot^{3}\right)$

\section{0,4}

379,7

$20,3^{3}$ )

.$^{3}$ )

500,4

$\left.1,7^{3}\right)$
$\left.\cdot{ }^{3}\right)$

1,7

502,1
92,0

399,4

461,4

1320,9

$14,3^{3}$ )

$122,7^{3}$ )

.$\left.^{3}\right)$

$\left.\cdot^{3}\right)$

2

24,6

10,3

$10,3^{3}$ )

$\left.\cdot^{3}\right)$

567,7

1843,0

$\left.\left.4{ }^{3}\right) \quad 24,6^{3}\right)$

4

2600,5

785,3

$\left.2,6{ }^{3}\right)$
$\left.\cdot{ }^{3}\right)$

$\left.14,6^{3}\right)$

14,6

2,6

1857,6

1) Die Zusammenführung der zwei Subsektoren in einer Tabelle erfolgte aus Geheimhaltungsgründen. Seit der F\&E-Erhebung 1998 sind die F\&E-Einrichtungen des Subsektors "Kraftwerksgesellschaften" im Subsektor "Firmeneigener Bereich" enthalten. Eine Zuordnung der F\&E-Einrichtungen des "firmeneigenen Bereichs" nach Wissenschaftszweigen ist nicht zielführend.

2) Einschließlich Österreichisches Forschungszentrum Seibersdorf.

3) Aus Geheimhaltungsgründen können die Daten nur gemeinsam ausgewiesen werden. Rundungsdifferenzen. 
Tabelle 26: FORSCHUNG UND EXPERIMENTELLE ENTWICKLUNG NACH WISSENSCHAFTSZWEIGEN

ZUSAMMENFASSUNG DER ERHEBUNGSBEREICHE UNTER AUSSCHLUSS DES FIRMENEIGENEN BEREICHS ${ }^{1}$ )

BESCHÄFTIGTE in F\&E (in Vollzeitäquivalent) in den Jahren 1989, 1993 und 1998

\begin{tabular}{|c|c|c|c|c|c|c|c|c|c|c|}
\hline & \multirow{3}{*}{ Wissenschaftszweige } & \multirow{2}{*}{\multicolumn{3}{|c|}{$\begin{array}{l}\text { Anzahl der F\&E } \\
\text { betreibenden } \\
\text { Erhebungseinheiten }\end{array}$}} & \multicolumn{6}{|c|}{ Beschäftigte in $F \& E$} \\
\hline & & & & & \multicolumn{2}{|c|}{1989} & \multicolumn{2}{|c|}{1993} & \multicolumn{2}{|c|}{1998} \\
\hline & & 1989 & 1993 & 1998 & in VZÄ & in $\%$ & in VZÄ & in $\%$ & in VZÄ & in $\%$ \\
\hline 1.0 & Naturwissenschaften & 245 & 266 & 269 & 2138,5 & 22,5 & 2590,4 & 24,2 & 3437,5 & 26,9 \\
\hline 2.0 & Technische Wissenschaften & 227 & 250 & 242 & 2034,7 & 21,4 & 2334,0 & 21,8 & 2731,0 & 21,4 \\
\hline 3.0 & Humanmedizin & 206 & 213 & 235 & 1764,9 & 18,6 & 2011,4 & 18,8 & 2563,3 & 20,1 \\
\hline 4.0 & $\begin{array}{l}\text { Land- } u \text {. Forstwirtschaft, } \\
\text { Veterinärmedizin }\end{array}$ & 89 & 92 & 78 & 1018,3 & 10,7 & 995,4 & 9,3 & 889,6 & 7,0 \\
\hline \multicolumn{2}{|r|}{ Zwischensumme } & 767 & 821 & 824 & 6956,4 & 73,2 & 7931,3 & 74,1 & 9621,3 & 75,3 \\
\hline 5.0 & Sozialwissenschaften & 244 & 316 & 327 & 1006,9 & 10,6 & 1448,4 & 13,5 & 1650,8 & 12,9 \\
\hline 6.0 & Geisteswissenschaften & 335 & 329 & 320 & 1533,6 & 16,1 & 1319,3 & 12,3 & 1508,4 & 11,8 \\
\hline \multicolumn{2}{|r|}{ Zwischensumme } & 579 & 645 & 647 & 2540,5 & 26,8 & 2767,8 & 25,9 & 3159,3 & 24,7 \\
\hline $1.0 \mathrm{t}$ & INSGESAMT & 1346 & 1466 & 1471 & 9496,9 & 100,0 & 10699,0 & 100,0 & 12780,6 & 100,0 \\
\hline
\end{tabular}
1) D.h. Zusammenfassung des Hochschulsektors, des Sektors Staat, des privaten gemeinnützigen Sektors sowie des kooperativen Bereichs und des Bereichs der Ziviltechniker, jedoch ohne firmeneigener Bereich (welcher die
Kraftwerksgesellschaften einschließt); ohne Landeskrankenanstalten. Eine Zuordnung des firmeneigegen Bereichs nach Wissenschaftszweigen ist nicht zielführend.

Rundungsdifferenzen. 
Tabelle 27: FORSCHUNG UND EXPERIMENTELLE ENTWICKLUNG IM FIRMENEIGENEN BEREICH 1998 ${ }^{1}$ )

Beschäftigte in F\&E und Ausgaben für F\&E, gegliedert nach Wirtschaftszweigen

\begin{tabular}{|c|c|c|c|c|c|c|c|}
\hline \multirow{2}{*}{$\begin{array}{l}\text { Lfd. Nr. } \\
\text { ISIC Rev.3 }\end{array}$} & \multirow{2}{*}{\multicolumn{3}{|c|}{$\begin{array}{c}\text { Wirtschaftszweige } \\
\text { (ÖNACE 1995-Abteilungen angeordnet } \\
\text { gemäß OECD/Frascati-Handbuch) }\end{array}$}} & \multicolumn{2}{|c|}{ Beschäftigte in F\&E } & \multicolumn{2}{|c|}{ Ausgaben für F\&E } \\
\hline & & & & in VZÄ & in \% & in $1000 \mathrm{~S}$ & in \% \\
\hline$<1>$ & $01+02+$ & & LAND- UND FORSTWIRTSCHAFT, FISCHEREI & G & $G$ & $\mathbf{G}$ & $G$ \\
\hline$<2>$ & $10-14$ & & BERGBAU, GEWINNUNG VON STEINEN UND ERDEN & $\mathbf{G}$ & $G$ & $\mathbf{G}$ & $G$ \\
\hline$<5>$ & & 15 & Nahrungs- und Genussmittel, Getränke & 201,6 & 1,1 & 250613 & 0,9 \\
\hline$<6>$ & & 16 & Tabakverarbeitung & G & $G$ & $\mathrm{G}$ & $G$ \\
\hline$<8>$ & & 17 & Textilien und Textilwaren (ohne Bekleidung) & 180,6 & 1,0 & 246141 & 0,9 \\
\hline$<9>$ & & 18 & Bekleidung & G & $G$ & $\mathrm{G}$ & $G$ \\
\hline$<10>$ & & 19 & Leder, Schuhe & 26,5 & 0,1 & 33322 & 0,1 \\
\hline$<12>$ & & 20 & Holz (ohne Möbel) & 70,5 & 0,4 & 136181 & 0,5 \\
\hline$<13>$ & & 21 & Papier und Pappe & 86,7 & 0,5 & 163401 & 0,6 \\
\hline \multirow[t]{2}{*}{$<14>$} & & 22 & Verlagswesen, Druckerei, Vervielfältigung von bespielten & & & & \\
\hline & & & Ton-, Bild- und Datenträgern & 21,7 & 0,1 & 50316 & 0,2 \\
\hline$<16>$ & & 23 & Kokerei, Mineralölverarbeitung & G & $G$ & G & G \\
\hline$<17>$ & & 24 & Chemikalien und chemische Erzeugnisse & $1.612,6$ & 8,7 & 2784123 & 10,3 \\
\hline$<20>$ & & 25 & Gummi- und Kunststoffwaren & 501,6 & 2,7 & 614170 & 2,3 \\
\hline$<21>$ & & 26 & Glas, Waren aus Steinen und Erden & 503,5 & 2,7 & 603413 & 2,2 \\
\hline$<22>$ & & 27 & Metallerzeugung und -bearbeitung & 466,9 & 2,5 & 785142 & 2,9 \\
\hline$<25>$ & & 28 & Metallerzeugnisse & 588,3 & 3,2 & 681478 & 2,5 \\
\hline$<27>$ & & 29 & Maschinenbau & $1.897,0$ & 10,2 & 2556640 & 9,4 \\
\hline$<28>$ & & 30 & $\begin{array}{l}\text { Büromaschinen, Datenverarbeitungsgeräte und } \\
\text {-einrichtungen }\end{array}$ & 70,7 & 0,4 & 63807 & 0,2 \\
\hline$<29>$ & & 31 & Geräte der Elektrizitätserzeugung, -verteilung, u.ä. & $1.119,2$ & 6,0 & 1329221 & 4,9 \\
\hline$<30>$ & & 32 & Rundfunk-, Fernseh- und Nachrichtentechnik & $5.172,3$ & 27,9 & 7920420 & 29,1 \\
\hline$<33>$ & & 33 & Medizin-, Mess-, Steuer- und Regelungstechnik, Optik & 587,0 & 3,2 & 655519 & 2,4 \\
\hline$<34>$ & & 34 & Kraftwagen und Kraftwagenteile & $1.544,1$ & 8,3 & 2520807 & 9,3 \\
\hline$<35>$ & & 35 & Sonstiger Fahrzeugbau & 362,3 & 2,0 & 782377 & 2,9 \\
\hline$<39>$ & & 36 & $\begin{array}{l}\text { Möbel, Schmuck, Musikinstrumente, Sportgeräte, } \\
\text { Spielwaren, sonstige Erzeugnisse }\end{array}$ & 229,5 & 1,2 & 276334 & 1,0 \\
\hline$<42>$ & & 37 & Rückgewinnung (Recycling) & - & - & - & - \\
\hline$<3>$ & $15-37$ & & SACHGÜTERERZEUGUNG & $15.425,3$ & 83,2 & 22730225 & 83,6 \\
\hline$<43>$ & $40+41$ & & ENERGIE- UND WASSERVERSORGUNG & 85,9 & 0,5 & 120361 & 0,4 \\
\hline$<44>$ & 45 & & BAUWESEN & 120,0 & 0,6 & 190086 & 0,7 \\
\hline$<46>$ & & $50-52$ & $\begin{array}{l}\text { Handel, Instandhaltung und Reparatur von Kfz und } \\
\text { Gebrauchsgütern }\end{array}$ & 546,4 & 2,9 & 762703 & 2,8 \\
\hline$<47>$ & & 55 & Beherbergungs- und Gaststättenwesen & - & - & - & - \\
\hline$<48>+<49>$ & & $60-64$ & Verkehr und Nachrichtenübermittlung & 382,5 & 2,1 & 315145 & 1,2 \\
\hline$<52>$ & & $65-67$ & Kredit- und Versicherungswesen & 196,3 & 1,1 & 374181 & 1,4 \\
\hline$<58>$ & & $70+71+74$ & $\begin{array}{l}\text { Realitätenwesen, Vermietung beweglicher Sachen, } \\
\text { unternehmensbezogene Dienstleistungen }\end{array}$ & 659,9 & 3,6 & 1026046 & 3,8 \\
\hline$<54>$ & & 72 & Datenverarbeitung und Datenbanken & 406,6 & 2,2 & 455804 & 1,7 \\
\hline$<57>$ & & 73 & Forschung und Entwicklung & 666,3 & 3,6 & 1141131 & 4,2 \\
\hline \multirow[t]{2}{*}{$<59>$} & & $75-93$ & $\begin{array}{l}\text { Öffentliche Verwaltung, Unterrichtswesen, Gesund- } \\
\text { heitswesen, sonstige öffentliche und persönliche }\end{array}$ & & & & \\
\hline & & & Dienstleistungen & 13,8 & 0,1 & 17720 & 0,1 \\
\hline$<45>$ & $50-93$ & & DIENSTLEISTUNGEN & $2.871,8$ & 15,6 & 4092730 & 15,2 \\
\hline$<60>$ & 01-93 & & INSGESAMT & $18.527,0$ & 100,0 & 27155939 & 100,0 \\
\hline
\end{tabular}




\section{Tabelle 28: $\quad$ FORSCHUNG UND EXPERIMENTELLE ENTWICKLUNG (F\&E) 1998 im INTERNATIONALEN VERGLEICH}

\begin{tabular}{|c|c|c|c|c|c|c|c|c|}
\hline \multirow{4}{*}{ Land } & \multirow{4}{*}{$\begin{array}{l}\text { Bruttoinlands- } \\
\text { ausgaben } \\
\text { für F\&E } \\
\text { in \% des BIP }\end{array}$} & \multirow{2}{*}{\multicolumn{2}{|c|}{$\begin{array}{l}\text { Finanzierung der } \\
\text { Bruttoinlandsausgaben } \\
\text { für F\&E durch }\end{array}$}} & \multirow{4}{*}{$\begin{array}{l}\text { Beschäftigte } \\
\text { in F\&E } \\
\text { in Vollzeit- } \\
\text { äquivalent }\end{array}$} & \multicolumn{4}{|c|}{ Bruttoausgaben für F\&E des } \\
\hline & & & & & \multirow{2}{*}{$\begin{array}{l}\text { Unternehmens- } \\
\text { sektors }\end{array}$} & \multirow{2}{*}{$\begin{array}{l}\text { Hochschul- } \\
\text { sektors }\end{array}$} & \multirow{2}{*}{ Sektors Staat } & \multirow{2}{*}{$\begin{array}{c}\text { privaten } \\
\text { gemeinnützigen } \\
\text { Sektors }\end{array}$} \\
\hline & & Staat & Wirtschaft & & & & & \\
\hline & & \multicolumn{2}{|c|}{ in \% } & & \multicolumn{4}{|c|}{ in \% der Bruttoinlandsausgaben für F\&E } \\
\hline Belgien $^{11}$ (15) & 1.84 & 24.9 & 69.4 & 39080.0 & 71.4 & 24.2 & 3.1 & 1.3 \\
\hline Dänemark & $1.93^{1)}$ & $36.1^{15)}$ & $53.4^{15)}$ & $\left.34083.0^{1)} 15\right)$ & $62.6^{11}$ & $21.3^{1)}$ & $15.2^{1)}$ & $1.0^{11}$ \\
\hline Deutschland & $2.31^{11}$ & $34.9^{11}$ & $62.3^{11}$ & $461539.0^{1)}$ & $67.9^{11}$ & $17.4^{1)}$ & $14.7^{1) 4)}$ & $.5)$ \\
\hline Finnland & 2.89 & 30.0 & $63.9^{14)}$ & 46517.0 & 67.2 & 19.6 & 12.6 & 0.6 \\
\hline Frankreich & 2.18 & 37.3 & 53.5 & 309515.0 & 62.3 & 17.6 & 18.6 & 1.5 \\
\hline Griechenland $^{15)}$ & 0.51 & 53.5 & 21.6 & 20173.0 & 25.6 & 50.6 & 23.4 & 0.4 \\
\hline Irland ${ }^{1 / 15)}$ & 1.39 & 22.2 & 69.2 & 12030.0 & 73.1 & 19.2 & 7.0 & 0.7 \\
\hline Italien & $1.02^{11)}$ & $51.1^{11)}$ & $43.9^{11)}$ & $141737.0^{15)}$ & $53.7^{11)}$ & $25.0^{11)}$ & $21.3^{11)}$ & . \\
\hline Niederlande & 1.95 & 37.9 & 48.6 & 85486.0 & 54.2 & 27.1 & 17.7 & 1.0 \\
\hline Osterreich & $1.81^{1)}$ & $39.2^{11}$ & $40.5^{11}$ & 31307,5 & $55.9^{3)}$ & $35.0^{3)}$ & $\left.8.9^{3)} 14\right)$ & $0.3^{3)^{14)}}$ \\
\hline Portugal ${ }^{15)}$ & 0.62 & 68.2 & 21.2 & 17999.0 & 22.5 & 40.0 & 24.2 & 13.3 \\
\hline Schweden ${ }^{15)}$ & 3.70 & 25.2 & 67.7 & 65495.0 & 74.8 & 21.5 & 3.5 & 0.1 \\
\hline Spanien & $0.90^{11}$ & $38.7^{11}$ & $49.8^{11}$ & $97098.0^{11}$ & $52.1^{11}$ & $30.5^{1)}$ & $16.3^{11}$ & $1.1^{1)}$ \\
\hline Vereinigtes Königreich & 1.83 & 31.1 & 47.3 & $270000.0^{31}$ & 65.8 & 19.5 & 13.4 & 1.3 \\
\hline EU insgesamt ${ }^{1 / 4}$ & $1.81^{11)}$ & $36.0^{11)}$ & $54.8^{11)}$ & $1607369.0^{11) 14) 15)}$ & $63.6^{11)}$ & $20.6^{11)}$ & $14.8^{11)}$ & 1.0 \\
\hline Island & $2.04^{11}$ & $55.9^{1)}$ & $37.7^{11}$ & $2273.0^{11}$ & $36.6^{11}$ & $24.9^{11}$ & $37.3^{11}$ & $1.2^{1)}$ \\
\hline Norwegen ${ }^{15)}$ & 1.66 & 42.9 & 49.4 & 24877.0 & 56.9 & 26.6 & $16.4^{4)}$ & $.5)$ \\
\hline Schweiz ${ }^{\text {y) }}$ & 2.73 & 26.9 & 67.5 & 50265.0 & 70.7 & 24.3 & $2.5^{7)}$ & 2.5 \\
\hline Polen & 0.73 & 59.0 & 37.8 & 84510.0 & 41.5 & 27.6 & 30.8 & 0.1 \\
\hline Tschechische Republik & 1.26 & $36.8^{14)}$ & 60.2 & 22740.0 & 64.6 & 9.5 & 25.7 & 0.2 \\
\hline Türkei ${ }^{15)}$ & 0.49 & 53.7 & 41.8 & 23432.0 & 32.3 & 57.2 & 10.5 & . \\
\hline Ungarn & 0.68 & $56.2^{13)}$ & $36.1^{13)}$ & 20315.0 & $38.4^{13)}$ & $25.2^{13)}$ & $31.2^{13)}$ & . \\
\hline Australien & 1.49 & 47.8 & 45.0 & 90717.0 & 45.1 & 29.4 & 23.4 & 2.1 \\
\hline Japan ${ }^{121}$ & 3.01 & 19.7 & 73.4 & 877162.0 & 71.9 & 14.0 & 9.3 & 4.8 \\
\hline Kanada & $1.62^{11)}$ & $\left.31.9^{11)} 12\right)$ & $48.7^{11)}$ & $137198.0^{2)}$ & $62.0^{11)}$ & $23.6^{11)}$ & $13.1^{11)}$ & $1.2^{11)}$ \\
\hline Mexiko & $0.34^{15)}$ & $71.1^{15)}$ & $16.9^{15)}$ & $33297.0^{2)}$ & $19.7^{15)}$ & $39.9^{15)}$ & $38.7^{15)}$ & $1.6^{15)}$ \\
\hline Korea & 2.55 & $22.9^{15)}$ & $72.5^{15)}$ & 128669.0 & 70.3 & 11.2 & 17.6 & 0.9 \\
\hline Neuseeland ${ }^{15)}$ & 1.13 & 52.3 & 30.5 & 12908.0 & 28.2 & 36.4 & 35.3 & . \\
\hline Skandi. Länder ${ }^{15)}$ & 2.63 & 31.8 & 61.5 & 167862.0 & 68.0 & 22.1 & 9.5 & $0.4^{12)}$ \\
\hline Vereinigte Staaten $^{11)}$ & $2.61^{6)}$ & $30.7^{6)}$ & $65.3^{6)}$ & . & $74.6^{6)}$ & $14.4^{6)}$ & $7.9^{7)}$ & $3.1^{6)}$ \\
\hline Nordamerika ${ }^{11)} 1<j$ & $2.22^{8)}$ & $31.2^{8)}$ & $63.9^{8)}$ & . & $73.3^{8)}$ & $15.1^{8)}$ & $8.6^{7)}$ & $2.9^{8)}$ \\
\hline OECD insgesamt ${ }^{11)}$ 12) & $2.18^{8)}$ & $30.7^{8)}$ & $62.5^{8)}$ & . & $69.2^{8)}$ & $17.1^{8)}$ & $11.1^{7)}$ & $2.6^{8)}$ \\
\hline
\end{tabular}

Q : OECD; Statistik Austria. $-{ }^{1)}$ Nationale Schätzung. $-{ }^{2)} 1995 .-{ }^{3)} 1993 .-{ }^{4)}$ Enthält auch andere Kategorien. $-{ }^{5)}$ Anderswo enthalten. $-{ }^{6)}$ Ohne Investitionsausgaben. $-{ }^{7)}$ Nur Bundesmittel oder Mittel der Zentralregierung. -- ${ }^{8)}$ Ohne Investitionsausgaben der Vereinigten Staaten. - ${ }^{9)}$ 1996. - ${ }^{10)}$ Nationale Erhebungsergebnisse (vom OECD-Sekretariat angepasste Werte). -- ${ }^{11)}$ Vorläufige Werte. -- ${ }^{12)}$ Schätzung des OECD-Sekretariates (basierend auf nationalen Quellen). --- ${ }^{13)}$ Die Summe der Gliederungselemente ergibt nicht die Gesamtsumme. -- ${ }^{14)}$ Bruch in der Zeitreihe. -- ${ }^{15)} 1997$. 
Tabelle 29: FWF: Bewilligungen nach Forschungsstätten 2000 (Mio. ATS)*

\begin{tabular}{|c|c|c|c|c|c|c|c|c|c|}
\hline Forschungsstätten & $\begin{array}{l}\text { Forschungs- } \\
\text { projekte }\end{array}$ & $\begin{array}{c}\text { SFB- } \\
\text { Teilprojekte }\end{array}$ & $\begin{array}{c}\text { FSP- } \\
\text { Projektteile }\end{array}$ & WKs & $\begin{array}{l}\text { Nachwuchs- } \\
\text { förderung** }\end{array}$ & $\begin{array}{l}\text { Druckkosten- } \\
\text { beiträge }\end{array}$ & $\begin{array}{l}\text { Anbah- } \\
\text { nungen }\end{array}$ & Summe & $\%$ \\
\hline \multicolumn{10}{|l|}{ a)Universitäre Forschungsstätten: } \\
\hline Universität Wien & 254,9 & 125,4 & 10,2 & 22,7 & 24,1 & 3,5 & 0,2 & 441,0 & 35,67 \\
\hline Universität Graz & 57,7 & 67,9 & 4,9 & & 7,7 & 0,4 & & 138,6 & 11,21 \\
\hline Universität Innsbruck & 94,0 & 1,4 & 3,4 & & 9,1 & 0,8 & 0,2 & 108,9 & 8,81 \\
\hline Universität Salzburg & 42,2 & 0,3 & 13,1 & & 0,9 & 0,1 & 0,1 & 56,7 & 4,59 \\
\hline Technische Universität Wien & 81,4 & 14,3 & 8,4 & 4,5 & 6,4 & 0,4 & 0,1 & 115,5 & 9,34 \\
\hline Technische Universität Graz & 43,9 & 41,6 & 5,2 & & 3,1 & 0,2 & & 94,0 & 7,60 \\
\hline Montanuniversität Leoben & 14,0 & & & & 1,7 & & & 15,7 & 1,27 \\
\hline Universität für Bodenkultur Wien & 48,4 & 9,0 & 2,5 & & 2,0 & 0,1 & & 62,0 & 5,01 \\
\hline Veterinärmedizinische Universität Wien & 12,1 & & 1,2 & & 0,3 & & & 13,6 & 1,10 \\
\hline Wirtschaftsuniversität Wien & 8,3 & 27,4 & & & 0,2 & 0,4 & & 36,3 & 2,94 \\
\hline Universität Linz & 19,1 & 9,1 & 0,1 & & 3,4 & & 0,2 & 31,9 & 2,58 \\
\hline Universität Klagenfurt & 3,0 & & & & & 0,6 & & 3,6 & 0,29 \\
\hline Universität für angewandte. Kunst Wien & 1,8 & & & & 1,0 & & & 2,8 & 0,23 \\
\hline $\begin{array}{l}\text { Universität für Musik und } \\
\text { darstellende Kunst Wien }\end{array}$ & 0,1 & & & & & & & 0,1 & 0,01 \\
\hline $\begin{array}{l}\text { Universität für Musik und } \\
\text { darstellende Kunst Graz }\end{array}$ & & & & & & 0,1 & & 0,1 & 0,01 \\
\hline b) Außeruniversitäre Forschungsstätten & & & & & & & & 0,0 & \\
\hline Österreichische Akademie der Wissenschaften & 53,6 & & 0,4 & & 0,5 & 0,9 & 0,4 & 55,8 & 4,51 \\
\hline Sonstige Forschungsstätten & 42,3 & 6,8 & 0,3 & & 6,9 & 3,3 & 0,3 & 59,9 & 4,84 \\
\hline Summe & 776,8 & 303,2 & 49,7 & 27,2 & 67,3 & 10,8 & 1,5 & $1.236,5$ & 100,00 \\
\hline
\end{tabular}

* Forschungsvorhaben, die gemeinsam an mehreren Forschungsstätten durchgeführt werden, wurden anteilsmäßig gewertet. Schrödingerstipendien wurden an der ursprünglichen Forschungsstätte der StipendiatInnen mit berücksichtigt.

** Schrödinger-, Meitner-, Bühler-Programm und Schrödinger-Rückkehrprogramm 
Tabelle 30: FWF: Bewilligungen nach Forschungsstätten 2000: Zahl der Neubewilligungen*

\begin{tabular}{|c|c|c|c|c|c|c|c|c|c|}
\hline Forschungsstätten & $\begin{array}{c}\text { Forschungs- } \\
\text { projekte }\end{array}$ & $\begin{array}{c}\text { FSP- } \\
\text { Teilprojekte }\end{array}$ & $\begin{array}{c}\text { SFB- } \\
\text { Projektteile }\end{array}$ & WKs & $\begin{array}{l}\text { Nachwuchs- } \\
\text { förderung** }\end{array}$ & $\begin{array}{c}\text { Druckkosten } \\
\text { beiträge }\end{array}$ & $\begin{array}{c}\text { An- } \\
\text { bahnungen }\end{array}$ & Summe & $\%$ \\
\hline \multicolumn{10}{|l|}{ a) Universitäre Forschungsstätten: } \\
\hline Universität Wien & 114,1 & 5,0 & 11,0 & 0,6 & 44,1 & 21,0 & 0,5 & 196,3 & 35,24 \\
\hline Universität Innsbruck & 36,2 & & & & 17,1 & 2,0 & 1,5 & 56,8 & 10,20 \\
\hline Universität Salzburg & 20,6 & 6,0 & & & 2,0 & & & 28,6 & 5,13 \\
\hline Technische Universität Wien & 34,9 & & 1,0 & 0,4 & 12,0 & 2,0 & & 50,3 & 9,03 \\
\hline Universität für Bodenkultur Wien & 19,8 & 1,0 & & & 3,0 & 1,0 & & 24,8 & 4,45 \\
\hline Veterinärmedizinische Universität Wien & 3,0 & & & & & & & 3,0 & 0,54 \\
\hline Wirtschaftsuniversität Wien & 3,8 & & & & & 3,0 & & 6,8 & 1,22 \\
\hline Universität Linz & 11,6 & & & & 4,0 & & 0,5 & 16,1 & 2,89 \\
\hline Universität Klagenfurt & 2,0 & & & & & 3,0 & & 5,0 & 0,90 \\
\hline Sonstige Forschungsstätten & 24,0 & & & & 9,8 & 19,0 & 1,5 & 54,3 & 9,75 \\
\hline Summe & 344,0 & 13,0 & 16,0 & 1,0 & 117,0 & 61,0 & 5,0 & 557,0 & 100,0 \\
\hline
\end{tabular}

* Forschungsvorhaben, die gemeinsam an mehreren Forschungsstätten durchgeführt werden, wurden anteilsgemäß gewertet. Schrödinger-Stipendien wurden an der ursprünglichen

Forschungsstätte der StipendiatInnen berücksichtigt. 
Tabelle 31: FWF:Förderungskategorien 2000: Neu- und Zusatzbewilligungen in Mio. ATS

1999

2000

\section{Förder-}

kategorien

Forschungsprojekte

Forschungsschwerpunkte (FSPs)

Spezialforschungsbereiche (SFBs)

Wissenschaftskollegs (WKs)

Erwin-Schrödinger-Stipendien

Lise-Meitner-Programm

Charlotte-Bühler-Programm

Erwin-Schrödinger-Rückkehrprogramm

Druckkostenbeiträge

Anbahnungen internationaler Kooperationen

Summe:

* inklusive Fortführung von FSPs, SFBs und WKs.

\section{Neube- Zusatzbe-}

willigungen* willigungen insgesamt

$\begin{array}{rrr}660 & 86,9 & 746,9 \\ 48,5 & 7,9 & 56,4 \\ 197,9 & 12,4 & 210,3 \\ 5,6 & 0 & 5,6 \\ 38,4 & 4,3 & 42,7 \\ 6,9 & 0,7 & 7,6 \\ 11,8 & 3,5 & 15,3 \\ & & \\ 10,7 & 0 & 10,7 \\ 1 & 0,3 & 1,3 \\ 980,8 & 116 & 1.096,80 \\ 89,42 \% & 10,58 \% & 100,00 \%\end{array}$

Neube- Zusatzbe-

$\%$ willigungen* willigungen insgesamt

$\%$

$\begin{array}{rrrrr}68,1 & 670,8 & 106 & 776,8 & 62,82 \\ 5,14 & 43,4 & 6,3 & 49,7 & 4,02 \\ 19,17 & 293,2 & 10 & 303,2 & 24,52 \\ 0,51 & 27 & 0,2 & 27,2 & 2,2 \\ 3,89 & 38,6 & 6,7 & 45,3 & 3,66 \\ 0,69 & 8,9 & 1,7 & 10,6 & 0,86 \\ 1,39 & 8 & 1,7 & 9,7 & 0,78 \\ & 1,7 & 0 & 1,7 & 0,14 \\ 0,98 & 10,5 & 0,3 & 10,8 & 0,87 \\ 0,12 & 1,2 & 0,3 & 1,5 & 0,12 \\ 100 & 1.103,30 & 133,2 & 1.236,50 & 100 \\ & 89,23 \% & 10,77 \% & 100,00 \% & \end{array}$


Tabelle 32: FWF: Bewilligungen nach Wissenschaftsdisziplinen 2000 (Mio. ATS)

Wissenschaftsdisziplinen

Naturwissenschaften

Technische Wissenschaften

Humanmedizin

Land- und Forstwirtschaft, Veterinärmedizin

Sozialwissenschaften

Geisteswissenschaften

Gesamt
1998

505,9

52

215

8,7

32,3

136,8

950,7
1999

$568,1 \quad 51,80 \%$

$79,7 \quad 7,27 \%$

$234,3 \quad 21,36 \%$

$17 \quad 1,55 \%$

$41,9 \quad 3,82 \%$

$155,8 \quad 14,20 \%$

$1.096,80 \quad 100,00 \%$
2000

$677,4 \quad 54,78 \%$

$51 \quad 4,12 \%$

$253,8 \quad 20,53 \%$

$16,6 \quad 1,34 \%$

$67,1 \quad 5,43 \%$

$170,6 \quad 13,80 \%$

$\begin{array}{rrrrr}14,39 \% & 155,8 & 17,6,6 & 13,80 \% \\ 100,00 \% & 1.096,80 & 100,00 \% & 1.236,50 & 100,00 \%\end{array}$


Tabelle 33: FFF: Förderungsübersicht 2000 nach Systematik der Wirtschaftstätigkeit (NACE)

\begin{tabular}{|c|c|c|c|c|c|c|c|c|}
\hline Fachbereich & NACE & $\begin{array}{l}\text { Zahl der } \\
\text { Projektes } \\
20 \text { do }\end{array}$ & $\begin{array}{l}\text { Zuerkannte } \\
\text { Förderungen* }\end{array}$ & $\begin{array}{l}\text { Förderungsmittel } \\
\text { Anteil } \\
\text { in öS } 1000 .\end{array}$ & $\begin{array}{c}\text { Barwert } \\
2000\end{array}$ & $\begin{array}{l}\text { Durchsch } \\
\text { Barwert pr }\end{array}$ & $\begin{array}{l}\text { licher } \\
\text { rojekt }\end{array}$ & \\
\hline Land- und Forstwirtschaft, Jagd & $1 / 2$ & $\frac{2000}{9}$ & $\frac{1999 i}{3}$ & $\frac{\text { in os 1.000,- }}{9.475}$ & & 2000 & $1999 \mathrm{ir}$ & 1.000 \\
\hline Kohlen-, Erz- und sonstiger Bergbau & $11 / 13 / 14$ & 10 & 7 & $\begin{array}{r}9.475 \\
17090\end{array}$ & $0,20 \%$ & $0,00 \%$ & 5.413 & 1.305 \\
\hline Herstellung von Nahrungs- und Genussmitteln und Getränken & 15 & 40 & 40 & 17.090 & $0,50 \%$ & $0,40 \%$ & 9.460 & 2.871 \\
\hline Textilien, Textilkwaren, Bekleidung & $17 / 18$ & 7 & 40 & 62.676 & $1,80 \%$ & $2,40 \%$ & 31.679 & 791 \\
\hline Ledererzeugung und -verarbeitung & 19 & 3 & & 38.768 & $1,10 \%$ & $2,40 \%$ & 12.985 & 3.531 \\
\hline $\mathrm{Be}-$ und Verarbeitung von Holz (ohne Herstellung von Möbeln) & 20 & 23 & $\begin{array}{r}3 \\
29\end{array}$ & 6.590 & $0,20 \%$ & $0,20 \%$ & 2.723 & 907 \\
\hline Herstellung und Verarbeitung von Papier und Pappe & 21 & 13 & 29 & 44.604 & $1,30 \%$ & $1,90 \%$ & 20.692 & 899 \\
\hline Verlagswesen, Druckerei, Vervielfältigung von bespielten Ton-, Bild- und Datentr... & 22 & 3 & $\begin{array}{r}16 \\
1\end{array}$ & 25.410 & $0,70 \%$ & $1,70 \%$ & 14.226 & 1.094 \\
\hline Kokerei, Mineralölverarbeitung, Herstellung und Verarbeitung von Spalt- und Brut.. & 23 & 2 & $\begin{array}{l}1 \\
2\end{array}$ & 12.230 & $0,30 \%$ & $0,40 \%$ & 4.540 & 1.513 \\
\hline Herstellung von Chemikalien und chemischen Erzeugnissen & 24 & 68 & $\begin{array}{r}2 \\
52\end{array}$ & 1.450 & $0,00 \%$ & $0,00 \%$ & 1.450 & 725 \\
\hline Herstellung von Gummi- und Kunststoffwaren & 25 & 37 & $\begin{array}{l}52 \\
34\end{array}$ & 534.425 & $15,10 \%$ & $15,60 \%$ & 254.248 & 3.738 \\
\hline Herstellung und Bearbeitung von Glas, Herstellung von Waren aus Steinen und Erde & 26 & 30 & 34 & 100.068 & $2,80 \%$ & $5,40 \%$ & 42.637 & 1.152 \\
\hline Metallerzeugung und -bearbeitung & 27 & 29 & 29 & 81.227 & $2,30 \%$ & $3,00 \%$ & 35.882 & 1.237 \\
\hline n Metallerzeugnissen & 28 & ${ }_{41}^{33}$ & 27 & 101.727 & $2,90 \%$ & $2,20 \%$ & 63.594 & 1.927 \\
\hline Maschinenbau & 29 & $\begin{array}{r}41 \\
146\end{array}$ & 33 & 89.905 & $2,50 \%$ & $3,10 \%$ & 36.969 & 901 \\
\hline Herstellung von Büromaschinen, Datenverarbeitungsgeräten und -einrictitungen & 30 & $\begin{array}{r}146 \\
6\end{array}$ & 133 & 459.111 & $13.00 \%$ & $14,30 \%$ & 201.100 & 1.377 \\
\hline Herstellung von Geräten der Elektrizitätserzeugung, -verteilung u.ä. & 31 & 6 & -9 & 26.040 & $0,70 \%$ & $0,70 \%$ & 11.576 & 1.929 \\
\hline Rundfunk-, Femseh- und Nachrichtentechnik & & 40 & 29 & 318.753 & $9,00 \%$ & $7,80 \%$ & 126.925 & 3.173 \\
\hline Medizin-, Mess-, Steuer- und Regelungstechnik, Optik & 32 & 67 & 55 & 516.143 & $14,60 \%$ & $13,10 \%$ & 226.591 & 3.381 \\
\hline $\begin{array}{l}\text { Herstellung von Kraftwagen und Kraftwagenteilen. } \\
\text { Hen }\end{array}$ & 33 & 81 & 69 & 300.198 & $8,60 \%$ & $6,80 \%$ & 136.241 & 1.681 \\
\hline Sonstiger Fahrzeugbau & $\begin{array}{l}34 \\
35\end{array}$ & 40 & 40 & 276.574 . & $7,80 \%$ & $6,40 \%$ & 141.413 & 3.535 \\
\hline Herstellung von Möbein, Schmuck, Musikinstrumenten, Sportgeräten, Splelwaren und... & $\begin{array}{l}35 \\
36\end{array}$ & 7 & 6 & 52.547 & $1,50 \%$ & $0,90 \%$ & 21.798 & 3.114 \\
\hline Rückgewinnung, Energie- und Wasserversorgung & $\begin{array}{r}36 \\
37 / 40 / 41\end{array}$ & 16 & 12 & 24.980 & $0,70 \%$ & $0,40 \%$ & 12.185 & 761 \\
\hline Bauwesen & & 3 & 0 & 8.870 & $0,20 \%$ & $0,00 \%$ & 3450 & 3450 \\
\hline Handelsvermittlung und Großhandel (ohne & $\begin{array}{l}45 \\
51\end{array}$ & 23 & 18 & 52.280 & $1,50 \%$ & $0,80 \%$ & 27.216 & 1.183 \\
\hline Einzelhandel (ohne Handel mit Kraftfahrzeugen) & $\begin{array}{l}51 \\
52\end{array}$ & 4 & 5 & 7.470 & $0,20 \%$ & $0,40 \%$ & 3.453 & 863 \\
\hline Datenverarbeitung und Datenbanken & $\begin{array}{l}52 \\
72\end{array}$ & 0 & 2 & 0 & $0,00 \%$ & $0,10 \%$ & 0 & 0 \\
\hline Forschung und Entwicklung & 72 & 107 & 79 & 300.344 & $8,40 \%$ & $7,90 \%$ & 146.386 & 1.368 \\
\hline Erbringung von unternehmensbezogenen Dienstleistungen & $\begin{array}{l}73 \\
.74\end{array}$ & 9 & 3 & 9.038 & $0,30 \%$ & $0,10 \%$ & 6.076 & 675 \\
\hline Gesundheit, Veterinär- und Sozialwesen & .74 & 6 & 6 & 4.065 & $0,10 \%$ & $0,10 \%$ & 4.065 . & 677 \\
\hline Abwasser- und Abfallbeseitigung und sonstige Entsorgung & $\begin{array}{l}85 \\
90\end{array}$ & .0 & 1 & & $0,00 \%$ & $0,00 \%$ & 0 & 0 \\
\hline SUMME & & $\frac{30}{903}$ & $\frac{23}{779}$ & $\frac{50.536}{3.532 .594}$ & $\frac{1,40 \%}{100 \%}$ & $\frac{1,40 \%}{99,70 \%}$ & 23.214 & 773 \\
\hline
\end{tabular}


Tabelle 34: FFF: Förderungsübersicht 2000 nach Sonderbereichen der Forschung

(Mehrfachnennungen möglich)

\section{Sonderbereich}

Biowissenschaften

Energietechnik

F\&E-Dynamik

Feasibility

Hochschule - Wirtschaft

Holzforschung

Lebensmittel-Initiative

Materialwissenschaften

Nachwuchsförderung

Umwelttechnik

Zulieferindustrie KFZ

*ind Haftungen, KOM, OeNB, EU

$\begin{array}{ccrccc}\begin{array}{c}\text { Zahl der } \\ \text { Projekte }\end{array} & \begin{array}{c}\text { Zuerkannte } \\ \text { Förderungen }\end{array} & \begin{array}{r}\text { Förderungs- } \\ \text { anteil 2000 }\end{array} & \begin{array}{c}\text { Förderungsmittel } \\ \text { Anteil }\end{array} & \begin{array}{c}\text { Barwert } \\ \text { Barwert } \\ \text { in \% }\end{array} \\ 28 & 422.210 & 14,80 \% & 12,80 \% & 204.390 & 14,60 \% \\ 13 & 93.627 & 3,30 \% & 4,90 \% & 44.898 & 3,20 \% \\ 23 & 62.761 & 2,20 \% & 1,10 \% & 24.290 & 1,70 \% \\ 174 & 2.149 & 0,10 \% & 0,00 \% & 2.149 & 0,20 \% \\ 43 & 675.004 & 23,70 \% & 23,20 \% & 340.082 & 24,30 \% \\ 64 & 76.102 & 2,70 \% & 4,10 \% & 40.040 & 2,90 \% \\ 97 & 107.733 & 3,80 \% & 3,70 \% & 53.927 & 3,80 \% \\ 42 & 340.605 & 12,00 \% & 11,00 \% & 161.630 & 11,50 \% \\ 72 & 72.025 & 2,50 \% & 2,90 \% & 40.647 & 2,90 \% \\ 47 & 209.259 & 7,30 \% & 7,80 \% & 100.492 & 7,20 \% \\ & 287.376 & 10,10 \% & 11,70 \% & 142.824 & 10,20 \%\end{array}$


Tabelle 35: FFF: Förderungsübersicht 2000 nach Bundesländern (Projektstandort)

\begin{tabular}{|c|c|c|c|c|c|c|c|}
\hline Bundesland & $\begin{array}{l}\text { Zahl der } \\
\text { Projekte }\end{array}$ & $\begin{array}{l}\text { Zahl der } \\
\text { Betriebe }\end{array}$ & $\begin{array}{c}\text { Gesamtförderung* } \\
\text { in öS 1.000,- }\end{array}$ & $\begin{array}{c}\text { Förderung } \\
2000\end{array}$ & $\begin{array}{c}\text { smittel Anteil } \\
1999 \\
\end{array}$ & $\begin{array}{c}\text { Banwert } \\
\text { in öS 1.000,- }\end{array}$ & $\begin{array}{c}\text { Barwert } \\
\text { in } \% \\
\end{array}$ \\
\hline Burgenland & 12 & 10 & 54.647 & $1,5 \%$ & $3,0 \%$ & 24.609 & $1,5 \%$ \\
\hline Kärnten & 65 & 53 & 320.303 & $9,1 \%$ & $9,3 \%$ & 127.775 & $7,8 \%$ \\
\hline Niederösterreich. & 127 & 104 & 338.021 & $9,6 \%$ & $9,1 \%$ & 162.090 & $10,0 \%$ \\
\hline Oberösterreich & 185 & 138 & 826.482 & $23,4 \%$ & $24,4 \%$ & 397.014 & $24,4 \%$ \\
\hline Salzburg & 48 & 41 & 159.831 & $4,5 \%$ & $3,9 \%$ & 68.075 & $4,2 \%$ \\
\hline Steiermark & 148 & 108 & 462.963 & $13,1 \%$ & $15,8 \%$ & 217.130 & $13,3 \%$ \\
\hline Tirol & 77 & 61 & 314.384 & $8,9 \%$ & $9,1 \%$ & 143.428 & $8,8 \%$ \\
\hline Vorarlberg. & 64 & 47 & 210.777 & $6,0 \%$ & $3,3 \%$ & 94.132 & $5,8 \%$ \\
\hline Wien & 177 & 121 & 845.186 & $23,9 \%$ & $22,2 \%$ & 393.947 & $24,2 \%$ \\
\hline SUMME & 903 & 677 & $3,532.594$ & $100,0 \%$ & $100,0 \%$ & 1,628.203: & $100,0 \%$ \\
\hline
\end{tabular}

*incl Haftungen, KOM, ONB, EU und Land. 


\section{Anhang II:}

\section{Szenarienrechnungen zur Erhöhung der F\&E-Quote des tip}

Im folgenden wird eine einfache Szenarienrechnung des tip dokumentiert, die den Fragen nachgeht, (i) welche Steigerungen der F\&E-Aufwendungen von öffentlicher Hand und von Privaten nötig sind, um das Regierungsziel einer Forschungsquote von 2,5\% im Jahr 2005 zu erreichen, (ii) welchen Beitrag zur Zielerreichung die für die nächsten 3 Jahre zusätzlich budgetierten öffentlichen Mittel leisten können.

Für diese (Neu-)Abschätzung ${ }^{49}$ des benötigten Mittelumfangs zur Erreichung einer F\&E-Quote von 2,5 $\%$ im Jahr 2005 wurde - auf Basis jüngster Daten des ÖSTAT (vergleiche Scholtze 2000) ${ }^{50}$ in bezug auf den derzeitigen Forschungsausgaben - von folgenden Annahmen ausgegangen: (i) nominelles Wachstum des BIP von durchschnittlich jährlich 4,5\% zwischen 2000 und 2005, (ii) laufende Erhöhung der

F\&E-Aufwendungen in konstanten prozentuellen Wachstumsraten.

\section{Basisszenario: Erhöhung der F\&E-Quote bis zum Jahr 2005}

Die Ergebnisse der Basisrechnung sind in Tabelle II-1 dargestellt. Der Referenzpfad umfasst die Ausgaben bei konstanter Forschungsquote von 1,78 \%. Aufgrund des BIP-Wachstums müssen schon zum Erreichen einer konstanten Quote auch die (nominellen) F\&E-Ausgaben laufend ansteigen - für eine Erhöhung der F\&E-Quote ist dementsprechend ein überproportionales Wachstum nötig. Im Basisszenario beträgt die nötige nominelle Wachstumsrate der F\&E-Ausgaben zur Erreichung des 2,5 $\%$-Ziels 11,8\%. Im Jahr 2005 müssten demnach in Österreich ca. 88 Mrd. ATS für F\&E aufgewendet werden. In Tabelle II-1 wird die schrittweise Annäherung der Forschungsquote an dieses Ziel dargestellt. Eine solche Annäherung macht eine laufende Steigerung der F\&E-Aufwendungen gegenüber dem Vorjahr notwendig (Spalte 4). Im Jahr 2001 müssten demnach gegenüber 2000 um 5,9 Mrd. ATS mehr an F\&E aufgewendet werden, um auf dem Pfad der Zielerreichung zu bleiben. Kumuliert über fünf Jahre machen die zusätzlich notwendigen Mittel um das Ziel einer Forschungsquote von 2,5 \% im Jahr 2005 schrittweise zu erreichen 68,4 Mrd. ATS aus.

Tabelle II-1: Erhöhung der Forschungsquote: Basis-Szenario

\begin{tabular}{|c|c|c|c|c|c|c|}
\hline & \multicolumn{2}{|c|}{ F\&E-Ausgaben } & \multirow{2}{*}{$\begin{array}{c}\text { F\&E- } \\
\text { Quote } \\
\text { Szenario }\end{array}$} & \multirow{2}{*}{$\begin{array}{c}\text { Steigerung der F\&E- } \\
\text { Ausgaben gegenüber } \\
\text { Vorjahr }\end{array}$} & \multirow{2}{*}{$\begin{array}{l}\text { Abweichen der F\&E- } \\
\text { Ausgaben vom Refe- } \\
\text { renzpfad }\end{array}$} & \multirow{2}{*}{$\begin{array}{c}\text { BIP zu laufenden } \\
\text { Preisen }\end{array}$} \\
\hline Jahr & Referenzpfad & Szenario & & & & \\
\hline 2000 & $50.205,7$ & $50.205,7$ & 1,78 & & 0,0 & $2.818 .100,0$ \\
\hline 2001 & $52.465,0$ & $56.143,3$ & 1,91 & $5.937,6$ & $3.678,4$ & $2.944 .914,5$ \\
\hline 2002 & $54.825,9$ & $62.783,1$ & 2,04 & $6.639,8$ & $7.957,3$ & $3.077 .435,7$ \\
\hline 2003 & $57.293,0$ & $70.208,2$ & 2,18 & $7.425,1$ & $12.915,2$ & $3.215 .920,3$ \\
\hline 2004 & $59.871,2$ & $78.511,4$ & 2,34 & $8.303,2$ & $18.640,2$ & $3.360 .636,7$ \\
\hline 2005 & $62.565,4$ & $87.796,6$ & 2,50 & $9.285,2$ & $25.231,2$ & $3.511 .865,3$ \\
\hline Summe & & & & & $68.422,2$ & \\
\hline
\end{tabular}

Quelle: Berechnungen von tip; Statistik Austria; WIFO; Joanneum Research, Angaben in Mio. ATS

In Tabelle II-2 ist dargestellt, wie sich diese notwendige Steigerung der globalen Ausgaben auf die einzelnen Finanzierungssektoren aufteilen kann, wobei von einer konstanten Finanzierungsstruktur

\footnotetext{
${ }^{49}$ Frühere Szenarienrechnungen, teilweise auf Basis älterer Daten bzw. anderer Annahmen, wurden vom WIFO wie auch von Gassler, Rammer, Polt (1999) vorgelegt.

${ }^{50}$ D.h. es wurden die offiziellen Angaben der Statistik Austria bezüglich der Globalschätzung der österreichischen Bruttoinlandsausgaben für F\&E herangezogen. Diese beinhalten die Finanzierung der in Österreich durchgeführten Forschung und experimentellen Entwicklung gemäß dem Frascati-Handbuch. Für eine Darstellung der Datengrundlagen vergleiche Scholtze 2000.
} 
(aktuell: öffentlicher Sektor: 38,2 \%; Unternehmen: 40,4 \%; privater gemeinnütziger Sektor: 0,4 \%; Ausland: $21,0 \%{ }^{51}$ ) über die gesamte Zeitperiode ausgegangen wurde.

Tabelle II-2: Aufteilung der notwendigen Finanzmittel auf unterschiedliche Finanzierungssektoren (in Mio. ATS)

Notwendige Ausgaben zur Erreichung des 2,5 \% - Ziels

\begin{tabular}{|c|c|c|c|c|c|c|c|c|}
\hline & \multirow[b]{2}{*}{ F\&E Ziel Reg. } & \multirow[b]{2}{*}{$\begin{array}{l}\text { F\&E-öffent- } \\
\text { licher Sektor }\end{array}$} & \multirow[b]{2}{*}{ Unternehmen } & \multirow[b]{2}{*}{$\begin{array}{l}\text { Privater } \\
\text { gemein- } \\
\text { nütziger } \\
\text { Sektor }\end{array}$} & \multirow[b]{2}{*}{ Ausland } & \multicolumn{3}{|c|}{ Aufgliederung öffentlicher Sektor } \\
\hline & & & & & & Bund & Bundesländer & Sonstige \\
\hline 2000 & $50.205,70$ & $19.188,5$ & $\begin{array}{l}20.286,7 \\
226859\end{array}$ & 180,0 & $10.550,5$ & $16.068,6$ & $\begin{array}{l}2.878,5 \\
32189\end{array}$ & $\begin{array}{l}214,4 \\
2398\end{array}$ \\
\hline 2001 & $56.143,31$ & $21.457,8$ & $22.685,9$ & 201,3 & $11.798,3$ & $17.969,0$ & $3.218,9$ & 239,8 \\
\hline 2002 & $62.783,13$ & $23.995,6$ & $25.368,9$ & 225,1 & $13.193,6$ & $20.094,1$ & $3.599,6$ & 268,1 \\
\hline 2003 & $70.208,21$ & $26.833,4$ & $28.369,1$ & 251,7 & $14.753,9$ & $22.470,5$ & $4.025,3$ & 299,8 \\
\hline 2004 & $78.511,43$ & $30.006,9$ & $31.724,2$ & 281,5 & $16.498,8$ & $25.128,0$ & $4.501,4$ & 335,3 \\
\hline 2005 & $87.796,63$ & $33.555,7$ & $35.476,1$ & 314,8 & $18.450,1$ & $28.099,8$ & $5.033,7$ & 374,9 \\
\hline
\end{tabular}

Quelle: Berechnungen von tip auf Basis von Daten der Statistik Austria

Unter der Annahme konstanter Finanzierungsanteile ergeben sich also im Jahr 2005 notwendige Ausgabenvolumina von 33,6 Mrd. ATS für den öffentlichen Sektor (davon 28,1 Mrd. ATS für den Bund), 35,5 Mrd. ATS für den Unternehmenssektor und 18,5 Mrd. ATS für den Sektor „Ausland“.

${ }^{51}$ Die Finanzierungsquelle Ausland umfasst - neben Zahlungen der EU im Rahmen der Rahmenprogramme für FTE - zum überwiegenden Teil Finanzierungen österreichischer F\&E in Unternehmen, die mit ausländischen Unternehmen verbunden sind (Scholtze 2000). In der neuen Erhebung der Statistik Austria ist dieser Finanzierungsanteil extrem hoch ausgewiesen. Österreich läge damit an der Spitze der OECD-Staaten. 


\section{Szenario: Impuls durch zusätzliche öffentliche F\& E-Ausgaben}

Um die Wirkung der aktuell budgetierten Erhöhungen der öffentlichen F\&E-Ausgaben abschätzen zu können, wurde in einer weiteren Szenariorechnung eine lineare Aufteilung der budgetierten sieben Mrd. ATS, die in den kommenden Jahren (2001 bis 2003) zusätzlich für Forschungsfinanzierung zur Verfügung stehen werden, vorgenommen ${ }^{52}$. Dabei wird unterstellt, dass die Mittel tatsächlich in vollem Umfang zusätzliche öffentliche F\&E-Ausgaben sind. Die Auswirkungen auf die Entwicklung der Forschungsquote sind in der letzten Spalte von Tabelle II-3 dargestellt. Ohne Berücksichtigung potentieller Hebelwirkungen ${ }^{53}$ bewirken die Forschungsmilliarden im Zeitraum von 2001 bis 2003 eine einmalige Anhebung der F\&E-Quote Österreichs von einem knappen Zehntelprozentpunkt.

Tabelle II-3: Auswirkungen der Forschungsmilliarden auf das sektorale Mittelaufkommen und die F\&EQuote (Referenzpfad plus aliquote Forschungsmilliarden, in Mio. ATS)

Forschungsmilliarden bis 2003

(ab 2001 lineare Aufteilung von 7 Mrd. ATS auf öff. Sektor)

\begin{tabular}{lcccccc} 
& öffentlicher Sektor & Unternehmen & $\begin{array}{c}\text { Privater gemeinnüt- } \\
\text { ziger Sektor }\end{array}$ & Ausland & Summe & $\begin{array}{c}\text { Entwicklung F\&E- } \\
\text { Quote }\end{array}$ \\
\hline 2000 & $19.188,5$ & $20.286,7$ & 180,0 & $10.550,5$ & $50.205,7$ & 1,78 \\
2001 & $22.385,3$ & $21.199,6$ & 188,1 & $11.025,3$ & $54.798,3$ & 1,86 \\
2002 & $23.287,7$ & $22.153,6$ & 196,6 & $11.521,4$ & $57.159,2$ & 1,86 \\
2003 & $24.230,6$ & $23.150,5$ & 205,4 & $12.039,9$ & $59.626,4$ & 1,85 \\
2004 & $22.882,6$ & $24.192,3$ & 214,7 & $12.581,7$ & $59.871,2$ & 1,78 \\
2005 & $23.912,4$ & $25.280,9$ & 224,3 & $13.147,8$ & $62.565,4$ & 1,78 \\
\hline
\end{tabular}

Quelle: Berechnungen von tip auf Basis von Daten der Statistik Austria

Ein Vergleich mit dem Basisszenario ermöglicht eine Einschätzung inwieweit die „neuen Forschungsmilliarden" ausreichen um das zur Zielerreichung notwendige Niveau (für den Ausgabenanteil des öffentlichen Sektors) zu erreichen. Für das Jahr 2001 ergeben sich inklusive dem aliquoten Anteil aus den Forschungsmilliarden knappe 22,4 Mrd. ATS für den öffentlichen Sektor, was um ca. 900 Mill. ATS über dem notwendigen Niveau liegt. Ab dem Jahr 2002 jedoch würden nach dieser Szenarienrechnung die Mittel der Forschungsmilliarden nicht mehr ausreichen, um den notwendigen Wachstumspfad zu halten.

Bezieht man - in Anlehnung an die Resultate der ökonometrischen Schätzungen für Österreich die einen "Hebel" von ca. 1,6 ${ }^{54}$ ergeben haben - die Hebelwirkung öffentlicher Ausgaben ein (vergleiche Kapitel 5.3.1. bzw. Tabelle II-4), dann wäre die Diskrepanz zwischen notwendigem und zu erwartendem Wachstumspfad zwar weniger stark ausgeprägt, aber auch dann würde das Ziel verfehlt werden.

\footnotetext{
${ }^{52}$ Die entspricht für das erste Jahr in etwa der tatsächlichen Entwicklung (vergleiche Kap. ÖSTAT 1.1)

${ }^{53}$ Und unter der Annahme, dass die Mittel der Forschungsmilliarden zur Gänze als F\&E-Aufwendungen im Sinne des FrascatiManual eingestuft werden können.

${ }^{54}$ D.h. ein zusätzlicher öffentlicher Forschungsschilling löst im Unternehmenssektor zusätzliche 0,6 F\&E-Schillinge aus
} 
Tabelle II-4: Entwicklung der Forschungsausgaben unter Annahme einer Hebelwirkung zusätzlicher öffentlicher F\&E-Aufwendungen von 1,6 (in Mio. ATS)

\begin{tabular}{cccccccc}
\hline & $\begin{array}{c}\text { F\&E- } \\
\text { offentlicher } \\
\text { Sektor }\end{array}$ & $\begin{array}{c}\text { Unternehmen } \\
\text { (inkl. „Hebel“) }\end{array}$ & $\begin{array}{c}\text { Privater gemein- } \\
\text { nütziger Sektor }\end{array}$ & Ausland & Summe & $\begin{array}{c}\text { Ohne weitere } \\
\text { Zielpfad } \\
\text { Ausgabensteige- } \\
\text { rungen }\end{array}$ \\
\hline 2000 & $19.188,5$ & $20.286,7$ & 180,0 & $10.550,5$ & $50.205,7$ & 1,78 & 1,78 \\
2001 & $22.385,3$ & $22.599,6$ & 188,1 & $11.025,3$ & $58.531,6$ & 1,91 & 1,91 \\
2002 & $23.287,7$ & $23.553,6$ & 196,6 & $11.521,4$ & $60.892,5$ & 1,90 & 2,04 \\
2003 & $24.230,6$ & $24.550,5$ & 205,4 & $12.039,9$ & $63.359,7$ & 1,90 & 2,18 \\
2004 & $22.882,6$ & $24.192,3$ & 214,7 & $12.581,7$ & $59.871,2$ & 1,78 & 2,34 \\
2005 & $23.912,4$ & $25.280,9$ & 224,3 & $13.147,8$ & $62.565,4$ & 1,78 & 2,50 \\
\hline
\end{tabular}

Quelle: Berechnungen von tip auf Basis von Daten der Statistik Austria

\section{Zusammenfassung und Schlussfolgerungen}

Die wichtigsten Resultate der obigen Szenarienrechnungen lassen sich wie folgt zusammenfassen:

- Das Regierungsziel ist sehr ehrgeizig und wird nur mit großen Anstrengungen zu erreichen sein.

- Seine Erreichung ist an schon kurzfristig einsetzende, aber dann nachhaltige und substantielle Erhöhungen der F\&E-Aufwendungen sowohl der öffentlichen Hand als auch der privaten Unternehmen geknüpft.

- Die aktuell budgetierten Mehrausgaben der öffentlichen Hand für die Jahre 2001-2003 bringen die F\&E-Quote kurzfristig (d.h. für das laufende Jahr) auf den notwendigen Wachstumspfad. Sie müssten zur Erreichung des Zieles allerdings schon im nächsten Jahr durch zusätzliche Mittel aufgestockt werden.

- Die notwendige Erhöhung der F\&E-Aufwendungen der Unternehmen kann durch eine Kombination von (a) Förderungen mit hoher Hebelwirkung, (b) Unterstützung raschen Strukturwandels in Richtung F\&E-intensive Branchen, (c) Attraktion F\&E-intensiver ausländischer Unternehmen angestrebt werden. Eine dieser Maßnahmen allein würde nicht ausreichen, zudem sind Strukturwandel und Unternehmensansiedlung Maßnahmen, die erst mittel- bis längerfristig größere Effekte aufweisen. 


\section{Anhang III/ 1: \\ Bericht der Projektgruppe „Hebung der Forschungsquote in der Wirtschaft“ vom 8. März 2001 an die Bundesregierung}

\section{Arbeitsauftrag}

Die Aufgabe der interministeriellen Projektgruppe bestand in der Erfüllung des Arbeitsauftrages, welcher im folgenden Absatz der Erklärung der Bundesregierung vom 11.7.2000 formuliert ist:

„Zur Hebung der Forschungsquote in der Wirtschaft werden die zuständigen Bundesministerien bis Jahresende 2000 geeignete gemeinsame Vorgehensweisen erarbeiten und der Bundesregierung die Setzung von stimulierenden Maßnahmen zur Steigerung der F\&E-Aktivitäten mit dem Ziel einer Erhöhung des Anteils der Wirtschaft an den gesamten F\&E-Finanzierung auf $60 \%$ vorschlagen: z.B. Ausbau der Kompetenzzentren (der Zahl und der Vernetzung in der Region nach); "matching funds", mit dem Bund, als Anreiz für betriebliche Forschung; Prüfung der neuen verstärkten indirekten staatlichen Förderungen zugunsten von Forschung und Technologie; gezielter Einsatz von Risikokapital.,,

\section{Zusammensetzung der Projektgruppe und Arbeitsweise}

Der Projektgruppe gehörten Vertreter folgender Institutionen an:

BMVIT, BMWA, BMBWK, Statistik Austria, FFF, TIG, Joanneum Research, WIFO

Leiter der Projektgruppe: Dr. Gerhard Kratky, BMVIT V/B

Gemäß den Anforderungen der Projektgruppe wurde vom BMVIT eine Studie mit dem Titel: „Möglichkeiten zur Erhöhung der Österreichischen Forschungsquote/Abschätzung der Effekte öffentlicher auf private F\&E-Ausgaben (Hutschenreiter - Polt)" in Auftrag gegeben.

Besondere Berücksichtigung fanden auch Dokumente der Europäischen Kommission, insbesondere "European Trend Chart on Innovation - Innovation Policy in Europe 2000" und in diesem Zusammenhang die dort angeführten good-practice-Beispiele zur Stärkung der Unternehmensforschung.

\section{Präambel zu den Empfehlungen}

Die Projektgruppe behandelt auftragsgemäß nur einen Teil des erforderlichen Maßnahmenkataloges, um die F\&E-Quote in Österreich anzuheben. Und zwar nur jenen Teil stimulierender Maßnahmen, die geeignet sind, kurz- und mittelfristig den Anteil der F\&E-Leistungen der Wirtschaft zu erhöhen. Wenn daher von der Projektgruppe Universitäten und sonstige die Grundlagenforschung (z.B. FWF, Akademie der Wissenschaften) betreffende Institutionen sowie Einrichtungen des höheren Bildungswesens (Fachhochschulen) nicht behandelt werden, soll deren wichtiger Beitrag für die F\&E-Quote und vor allem deren langfristiger Einfluss auch auf die F\&E-Leistungen in der Wirtschaft nicht in Abrede gestellt werden.

Die Steigerung der F\&E-Quote in der Wirtschaft ist nicht zuletzt vor dem Hintergrund der Gewährleistung einer nachhaltigen ökologischen und sozioökonomischen Entwicklung in Österreich zu sehen. Die Programme und Fördermaßnahmen der Bundesregierung zur Steigerung der F\&E-Quote müssen die sozioökonomischen Rahmenbedingungen von F\&E, sowie damit zusammenhängende Fragen der Umwelt- und Lebensqualität sowie des globalen Wandels als integralen Bestandteil mit berücksichtigen. Nur so kann die Treffsicherheit der Maßnahmen der Bundesregierung im Lichte der übergeordneten forschungs- und technologiepolitischen Ziele gewährleistet werden. 
Für die Hebung der F\&E-Quote ist neben den Förderungsmaßnahmen die Verbesserung der rechtlichen und wirtschaftlichen Rahmenbedingungen von ausschlaggebender Bedeutung. Da sich mit diesem Themenkreis eine eigene Projektgruppe („Überprüfung der innovationsfördernden bzw. hemmenden Rahmenbedingungen") befasst, wurde dieser Bereich trotz seiner Wichtigkeit hier nicht behandelt.

Die Projektgruppe ist auch immer von der Annahme ausgegangen, dass die Input-orientierte Zielsetzung „2,5 \% F\&E-Quote im Jahre 2005" nur eine Kurzformel darstellt und immer im Zusammenhang mit der - ebenfalls in der Erklärung der Bundesregierung proklamierten - Umstellung auf die „OutputOrientierung,, zu sehen ist. Nämlich die Überzeugung, dass nur durch Innovationen und innen zugrunde liegende Forschungsaktivitäten die Steigerung der Wertschöpfung und der Beschäftigung sowie eine Verbesserung der internationalen Konkurrenzfähigkeit des Wirtschaftsstandortes Österreich erreichbar ist. Die Projektgruppe beschränkte sich daher nicht auf quantitative Überlegungen auf der Input-Seite, sondern behandelte auch qualitative Beurteilungen auf der Output-Seite, also insbesondere die Frage, durch welche Maßnahmen das Angebot an innovativen Produkten und Verfahren erhöht und deren Umsetzung in Wirtschaftsleistung beschleunigt werden kann.

\section{Empfehlungen der Projektgruppe an die Bundesregierung}

Öffentliche Förderung sollte auf eine Hebelwirkung abzielen, durch welche eine Steigerung privater F\&E-Ausgaben über das vor der Förderung geplante Ausmaß hinaus herbeigeführt wird. Additionalität ist dabei ein zentrales Kriterium. Förderungsinstrumente, bei welchen hohe Mitnahmeeffekte zu erwarten sind, sind abzulehnen bzw. sind besonders kritisch auf die Erreichung anderer Wirkungen (z.B. erwünschte Struktureffekte) zu hinterfragen.

Die im folgenden genannten Ergebnisse internationaler Studien sollten Einfluss auf die Forschungsund Technologiepolitik in Österreich haben:

- Die Hebelwirkung steigt mit dem technologischen und kommerziellen Risiko sowie mit der Langfristigkeit der Projekte.

- $\quad$ Bei KMUs ist die Hebelwirkung im allgemeinen höher als bei Großunternehmen. Das Volumen der Forschungsausgaben insgesamt wird jedoch in hohem Ausmaß von den Forschungsaktivitäten der Großunternehmen bestimmt.

- Je marktnäher die Forschung, umso wichtiger ist die Absicherung gegen Mitnahmeeffekte durch entsprechendes Förderungsdesign.

- $\quad$ Direkte Förderungen wirken nachhaltiger und meist effektiver als indirekte (steuerliche) Förderungen und haben geringere Mitnahmeeffekte.

- $\quad$ Förderungen können sich in ihrer Wirkung gegenseitig beeinträchtigen und müssen aufeinander abgestimmt werden. Dies gilt sowohl für direkte vs. indirekte Förderung als auch für verschiedene Instrumente der direkten Förderung.

- $\quad$ Unter den Instrumenten der direkten Förderung stimulieren Kapital- und Erfolgsbeteiligung die F\&E-Ausgaben der Unternehmen überproportional.

Im Zuge einer von der Projektgruppe vorgenommenen Qualifizierung und Analyse der wichtigsten bestehenden und geplanten Förderungsinstrumente in standisiertem Format ergaben sich für das Nationale Innovationssystem folgende Befunde: 
- $\quad$ Die aktuell vorgeschlagenen Programme zur Förderung der F\&E in der Wirtschaft zielen von ihrer Anlage her durchwegs auf eine hohe Hebelwirkung ab. In ihrer überwiegenden Mehrzahl wirken sie auch schon kurzfristig erhöhend auf die F\&E-Quote.

- $\quad$ Durch das Spektrum dieser Förderungen wird den wichtigsten Problemen des Österreichischen Innovationssystem (z.B. Kooperation Wissenschaft-Wirtschaft, Erhöhung des Innovationsniveaus der österreichischen Unternehmen) begegnet.

- Zusätzliche Akzentuierungen könnten noch in den Bereichen „Gründungsdynamik, sowie „Wirtschaft-Wirtschaft -Kooperationen/Cluster,, gesetzt werden.

Aus den internationalen Studien und der Analyse des nationalen Innovationssystems ergeben sich folgende weiteren Empfehlungen:

- $\quad$ Der Forschungsfreibetrag ist in Österreich bereits heute im internationalen Vergleich relativ hoch und sollte in dieser Form nicht unbedingt weiter ausgebaut werden. Eine Reform der steuerlichen Förderung sollte in die Richtung gehen, auch im Aufbau befindliche Unternehmen profitieren zu lassen (z.B. Absetzbetrag, Begünstigung von F\&E-Personal).

- Zeitlich befristete Kapitalbeteiligungen mit hoher Risikokomponente sind besonders erfolgversprechend, weil sie primär für Projekte in Anspruch genommen werden, die ohne Förderung nicht durchgeführt worden wären. Kapitalbeteiligungen in der Frühphase eines Unternehmens haben auch den Vorteil, die Eigenkapitalstruktur zu verbessern und damit als Finanzierungshebel zu wirken. Ein solches Finanzierungsprogramm sollte als zusätzliches Instrument neben den bestehenden Förderansätzen aufgebaut werden.

- $\quad$ Förderungspolitik muss immer auch als Strukturpolitik verstanden werden. Neben den bewährten bottom-up Instrumenten - in Österreich im Wirtschaftsbereich insbesondere der FFF - bedarf es daher auch geeigneter top-down Instrumente, um einen Strukturwandel in Richtung innovationsstarker und humankapitalintensiver Bereiche bzw. Projekte zu erreichen. Ansatzpunkte für Schwerpunktsetzungen in der Förderpolitik sollten Technologiefelder mit breiter Ausstrahlung in viele Wirtschaftsbereiche (z.B. eCommerce), die Bildung/Stärkung von Clustern (z.B. Biotechnologie, Wasser) und Themen mit hohen gesellschaftlichen Erträgen (z.B. Nachhaltigkeit und Mobilität) sein.

- $\quad$ Ein entscheidender Erfolgsfaktor für die Erhöhung der F\&E-Quote und des Innovationsniveaus der österreichischen Unternehmen in Richtung grundlegenderer, längerfristigerer und riskanterer Projekte liegt darin, ob und inwieweit es gelingt, das Forschungspotential der Wissenschaft für die Wirtschaft verfügbar zu machen. Diesbezügliche Kooperationsprogramme (z.B. Kompetenzzentren und Christian Doppler Laboratorien) haben sich bisher bewährt und sollen weitergeführt, ausgebaut bzw. ergänzt und besser aufeinander abgestimmt werden.

- $\quad$ Die begleitende und nachträgliche Erfolgskontrolle (Monitoring und Evaluierung) aller laufenden und neuen Programme ist auszubauen. Für zusätzliche F\&E-Mittel und neue Programme ist die Untersuchung ihrer quantitativen und qualitativen Effekte (nach dem Vorbild Finnlands) vorzusehen. Eine grundlegende Voraussetzung dafür ist die Durchführung von regelmäßigen statistischen Erhebungen in allen volkswirtschaftlichen Sektoren auf der Basis der international geltenden Richtlinien und in Zeitabständen, die dem internationalen und von der EU geforderten Standard entsprechen.

- Unsicherheit der Unternehmen bezüglich der künftigen Förderungspolitik (z.B. durch ständige Veränderung der Förderungsbedingungen, der Förderintensitäten ) vermindert deren Effektivität. Aus diesem Grund ist eine längerfristige Orientierung und Finanzierungsgrundlage der För- 
derungspolitik zu empfehlen. Ausbau und Neuorientierung bestehender Einrichtungen und Aktivitäten ist der Vorzug zu geben vor der additiven Neugründung.

Der Mix aus bestehenden und geplanten Förderungsinstrumenten erscheint geeignet, die von der Bundesregierung vorgegebenen qualitativen Ziele, vor allem die Hebelwirkung in die Wirtschaft, zu erreichen. Das budgetierte und derzeit vorgesehene Fördervolumen ist bei weitem nicht ausreichend, das quantitative Ziel (2,5\% am BIP bis 2005) zu erreichen. 


\section{Anhang III/2:}

\section{Statistische Erhebungen - Synchronisierung mit internationalen Organisationen}

Entsprechend der Erklärung der Bundesregierung vom 11. Juli 2000 zu aktuellen Fragen der Forschungs- und Technologiepolitik werden die betroffenen Bundesministerien bzw. wird die Bundesregierung folgende Maßnahmen setzen, um sicherzustellen, dass beginnend mit dem Basiserhebungsjahr 2000 regelmäßige umfassende statistische Erhebungen über Forschung, Technologie und Innovation in dem internationalen Standard entsprechenden Zeitabständen in allen volkswirtschaftlichen Sektoren durch Statistik Österreich durchgeführt werden:

- $\quad$ Beauftragung der Statistik Österreich mit regelmäßigen umfassenden Erhebungen über Forschung und experimentelle Entwicklung (F\&E) in allen volkswirtschaftlichen Sektoren auf der Basis der international und im EU-Bereich geltenden Richtlinien, Standards und Definitionen (insbesondere des Frascati-Handbuches) in 2-Jahres-Intervallen. Die erste dieser Erhebungen wird über das Berichtsjahr 2001 durchgeführt werden, wodurch ein Einschwenken auf den 2Jahres-Rhythmus der ISY-Erhebungen der OECD (ungerade Jahre) erreicht wird. Die Beauftragung der Statistik Österreich mit dieser und den darauf folgenden in 2-Jahres-Abständen durchzuführenden F\&E-Erhebungen wird durch eine Verordnung (der Bundesregierung oder der schwerpunktmäßig am meisten betroffenen Bundesminister) auf der Grundlage des Bundesstatistikgesetzes 2000 erfolgen.

- Beauftragung der Statistik Österreich mit der Durchführung der 3. Europäischen Innovationserhebung (CIS 3) im Unternehmenssektor in Österreich über das Berichtsjahr 2000, welche in der zweiten Jahreshälfte 2001 auf der Basis einer vertraglichen Beauftragung durch das BMWA und eines Vertrages mit EUROSTAT durchgeführt werden wird. Entsprechend den Datenanforderungen der EU und der OECD werden Innovationserhebungen im Unternehmenssektor - gemäß dem auf dem Oslo-Handbuch basierenden Europäischen CIS-Konzept - künftig in den Jahren zwischen den F\&E-Erhebungen, somit gleichfalls in 2-Jahres-Abständen, durchgeführt werden. Im Rahmen dieser Erhebungen werden auch einzelne Eckdaten über F\&E (zumindest Insgesamt-Ausgaben für F\&E) erfragt, wodurch eine gewisse Datenbasis für die für die NichtF\&E-Erhebungsjahre von Statistik Österreich zu erstellenden Schätzungen der nationalen F\&EAufwendungen, welche für die jährliche Berichterstattung an EUROSTAT benötigt werden, verfügbar sind. In Einklang mit entsprechenden Überlegungen auf EU-Ebene wird gegebenenfalls jeweils jede zweite Innovationserhebung (also erstmals die vorgesehene Erhebung über das Berichtsjahr 2002) mit reduziertem Umfang durchgeführt werden.

- $\quad$ Angesichts der Beschlüsse des EU-Rates zu „e-Europe“ und der entsprechenden österreichischen Aktivitäten betreffend „e-Austria“ kommt der Schaffung einer international vergleichbaren standardisierten statistischen Datenbasis über die Phänomene der Informationsgesellschaft hohe Priorität zu („e-statistics for e-Europe“). In das Europäische Statistische System (ESS) eingebettete, d.h. mit den bestehenden Wirtschafts- und Sozialstatistiken vergleichbare, Statistiken über die Informationsgesellschaft sind im Aufbau.

Im Rahmen dieser EU-Initiativen wird die erste harmonisierte Europäische Piloterhebung über E-Commerce durchgeführt. Die Statistik Österreich ist vom BMWA vertraglich beauftragt worden, diese Erhebung auf der Grundlage des von EUROSTAT vorgegebenen Fragenprogramms über den Berichtszeitraum 2000/2001 in Österreich durchzuführen. Sie findet im ersten Halbjahr 2001 statt und umfasst sowohl den Produzierenden Bereich als auch den Dienstleistungsbereich. Ein Vertrag zwischen der Statistik Österreich und EUROSTAT betreffend einen Förderungszuschuss seitens der Kommission ist abgeschlossen.

Mit den vorgesehenen bzw. bereits erfolgten Maßnahmen wird eine Konsolidierung der Statistiken über Forschung, Technologie und Innovation angestrebt, die es in Zukunft möglich machen wird, dass 
sowohl für Politikentscheidungen auf nationaler wie supranationaler Ebene als auch für die nationale Berichterstattung und die Berichterstattung an EU und OECD sowie für die wissenschaftliche Forschung die benötigten statistischen Grundlagen regelmäßig auf der Basis von periodisch durchgeführten statistischen Erhebungen im Rahmen des nationalen und des Europäischen Statistischen Systems zur Verfügung stehen werden. 


\section{Anhang III/3:}

\section{Erweiterung der Bund/ Bundesländer-Kooperation}

In der „Erklärung der Bundesregierung zu aktuellen Fragen der Forschungs- und Technologiepolitik“, vom 11. Juli 2000 wird mit Bezug auf das Ziel der Bundesregierung, den Anteil der Ausgaben für Forschung und Entwicklung anzuheben, vorgeschlagen: „U. a. zur Hebung der Forschungsquote in den Ländern ist die seit 1984 eingerichtete Bund/Bundesländerkooperation auf eine neue Basis zu stellen.“

Die Bund/Bundesländer-Kooperation hat sich im Bereich der Forschung, Entwicklung und Innovation als Instrument eines "gelebten Forschungsföderalismus“ bewährt; aufgrund ihrer Organisationsstruktur ist sie fähig, geänderten gesellschaftlichen Bedürfnissen durch die Berücksichtigung neuer Aufgabenfelder Rechnung zu tragen.

Diese Kooperation hat, gestützt auf eine flexible Organisation, die ihr übertragene Aufgabe, im gemeinsamen Interesse von Ländern und Bund gelegene Forschungsvorhaben gemeinsam zu finanzieren und durchzuführen, in hohem Maße für alle teilnehmenden Partner erfüllt.

Die Kooperation wurde von den zu Beginn ihrer Tätigkeit intendierten Schwerpunktsetzungen, in den Bereichen der Rohstoffforschung und der Energieforschung, erfolgreich auf die Bereiche der Erforschung der unterirdischen Wasservorräte, der nachwachsenden Rohstoffe, der Forschung und Entwicklung zu alternativen Energieträgern, insgesamt der Umweltforschung (unter Einschluss der Kulturlandschaftsforschung) mit der Zielvorgabe einer nachhaltigen Entwicklung erweitert.

Anlässlich der 23. Arbeitstagung der Bund/Bundesländer-Kooperation, die auf Einladung der oberösterreichischen Landesregierung am 12./13. Oktober 2000 in Aigen-Schlägl stattfand, wurde die Weiterentwicklung der Bund/Bundesländer-Kooperation von den Landesvertretern ausführlich diskutiert.

Aufbauend auf den bisherigen Erfahrungen dieser Kooperation ist es im Sinne der „Erklärung der Bundesregierung" erforderlich, den erfolgreichen Weg des kooperativen Forschungsföderalismus im Rahmen der Bund/Bundesländer-Kooperation thematisch, strukturell und organisatorisch auszubauen.

- Hebung der Attraktivität und Leistungsfähigkeit des Forschungsstandortes Österreich durch Stärkung der Kooperation zwischen Bund und Bundesländern;

- Stimulierung von Wissenschaft und Wirtschaft innerhalb der Regionen; Sicherung regionaler Standorte durch Kooperation zwischen Wirtschaft und Wissenschaft;

- Stärkung der Forschungskompetenz und Forschungskapazität in den Bundesländern;

- Stärkung der Durchlässigkeit des universitären und außeruniversitären Sektors in den Regionen in Richtung Wirtschaft ;

- Nutzung regionaler universitärer und außeruniversitärer Kompetenzen durch gemeinsame Einrichtung bzw. Ausbau von Forschungsinstitutionen, Fachhochschulen, Kompetenzzentren und Technologietransferzentren als Inkubatoren;

- Einrichtung von regionalen (überregionalen) Kooperations- und Informationsnetzwerken zwischen universitären, außeruniversitären und industriellen Forschungseinrichtungen;

- Vernetzung von Forschungsschwerpunkten der Bundesregierung mit den entsprechenden Schwerpunktssetzungen der Bundesländer;

- Einbindung der Forschungs- und Entwicklungsinitiativen der Bundesländer in Aktivitäten der Bildung eines „Europäischen Forschungsraums". 
Die thematischen Schwerpunktsetzungen der Bund/Bundesländer-Kooperation sollen dabei auf neue, zukunftsfähige Bereiche gemeinsamen Interesses unter Berücksichtigung des gesamten F\&EPotentials des Bundes und der Länder erweitert werden.

Gemeinsames Ziel von Bund und Ländern ist es, durch diese Erweiterung und Intensivierung der Zusammenarbeit im Bereich der Forschung, Entwicklung und Innovation zur Förderung von in beiderseitigem Interesse gelegenen Bereichen Synergien zu nutzen und zur Stärkung des Forschungsstandorts Österreich beizutragen. 


\section{Anhang III/4:}

\section{Programm "Public Understanding of Science and Technology"}

Die "Erklärung der Bundesregierung zu aktuellen Fragen der Forschungs- und Technologiepolitik" vom 11. Juli 2000 hat die Vorbereitung eines Programms "Public Understanding of Science and Technology" als Ziel der Bundesregierung avisiert.

In interministerieller Abstimmung haben die zuständigen Bundesministerien jeweils Aktionen, die dieser Thematik in ihrem Ressortbereich zuzuordnen sind, vorgestellt. Das BMBWK hat als Beitrag ein spezifisches Programm vorbereitet, das im Einklang mit den Überlegungen zur Vorbereitung eines Europäischen Forschungsraums konzipiert worden ist. Es ist auf Synergien zwischen Bildung -Jugend - Schule - Wissenschaft - Forschung - Kultur hin orientiert.

Die Förderung des Interesses der Jugend und der SchülerInnen für Wissenschaft und Forschung trägt zur Wahl von wissenschaftlichen Karrieren und für die Ausbildung für künftige WissenschaftsArbeitsplätze in hohem Maße bei. Dies ist eine Voraussetzung in einer sich herausbildenden "Wissensgesellschaft. Zugleich und in gleichem Maße bedarf es auch einer stärkeren Verankerung von "Wissenschaftsbewusstsein" in einem möglichst breiten Segment der Bevölkerung. Akzeptanz und Verständnis für Wissenschaft und Forschung sind Teil einer lebendigen Kultur und nicht zuletzt ein Ausdruck einer auf mündigen Bürgerinnen und Bürgern beruhenden Demokratie. Dazu bedarf es eines kontinuierlichen Dialoges zwischen der Welt der Wissenschaft und den verschiedenen gesellschaftlichen Akteuren. Diesen Dialog zu fördern und auszubauen, ist Ziel des Programms des BMBWK.

\section{Folgende Schwerpunkte sind vorgesehen:}

- Bewusstmachen der fundamentalen Rolle von Wissenschaft und Forschung in allen Bereichen des täglichen Lebens

- $\quad$ Steigerung des Interesses der Jugend an Wissenschafts- und Forschungsfragen und Steigerung der Motivation von SchülerInnen der Sekundarstufe zur Wahl (natur)wissenschaftlichtechnischer Fächer

- $\quad$ Steigerung der Kommunikationsfähigkeit von WissenschafterInnen und Laien in beiden Richtungen mit dem Ziel einer Schaffung von Kommunikationsnetzwerken, Erhöhung der Fähigkeit zur Diskussion wissenschaftsnaher Fragestellungen des täglichen Lebens

- $\quad$ Förderung von "Wissenschaftskommunikatoren" in Forschungseinrichtungen, in den Print- und elektronischen Medien, in Schulen und in der Verwaltung

Das Programm ist modular aufgebaut und wird in Abstimmung mit Aktivitäten auf europäischer Ebene umgesetzt. Die einzelnen Aktionsbereiche sollen einander ergänzen, aber jeweils für sich selbst entwickelt und durchgeführt werden. Internationale Vernetzung von Trägern und Aktivitäten wird durch das Programm ausdrücklich gefördert. Laufendes Monitoring und Evaluierung in mittelfristigen Abständen sind zur Überprüfung der Wirksamkeit der einzelnen Module und Aktionsbereiche vorgesehen. Die Finanzierung soll komplementär aus öffentlichen und privaten Mitteln (Sponsoring) erfolgen und den Aufbau eines spezifischen Anreizsystems zur Förderung der Dialogbereitschaft vor allem der Wissenschaftseinrichtungen ermöglichen. Damit wird ein breit gefächertes zielgruppenspezifisches Angebot von lokalen bis regionalen und bundesweiten Aktivitäten und Maßnahmen mit Modellcharakter entwickelt werden. Begleitende Forschung ist zur Professionalisierung der Aktivitäten und zur Erhöhung von Effektivität und Effizienz des Programms vorgesehen. 